\title{
Water-Chemistry Data for Selected Springs, Geysers, and Streams in Yellowstone National Park, Wyoming, 2006-2008
}

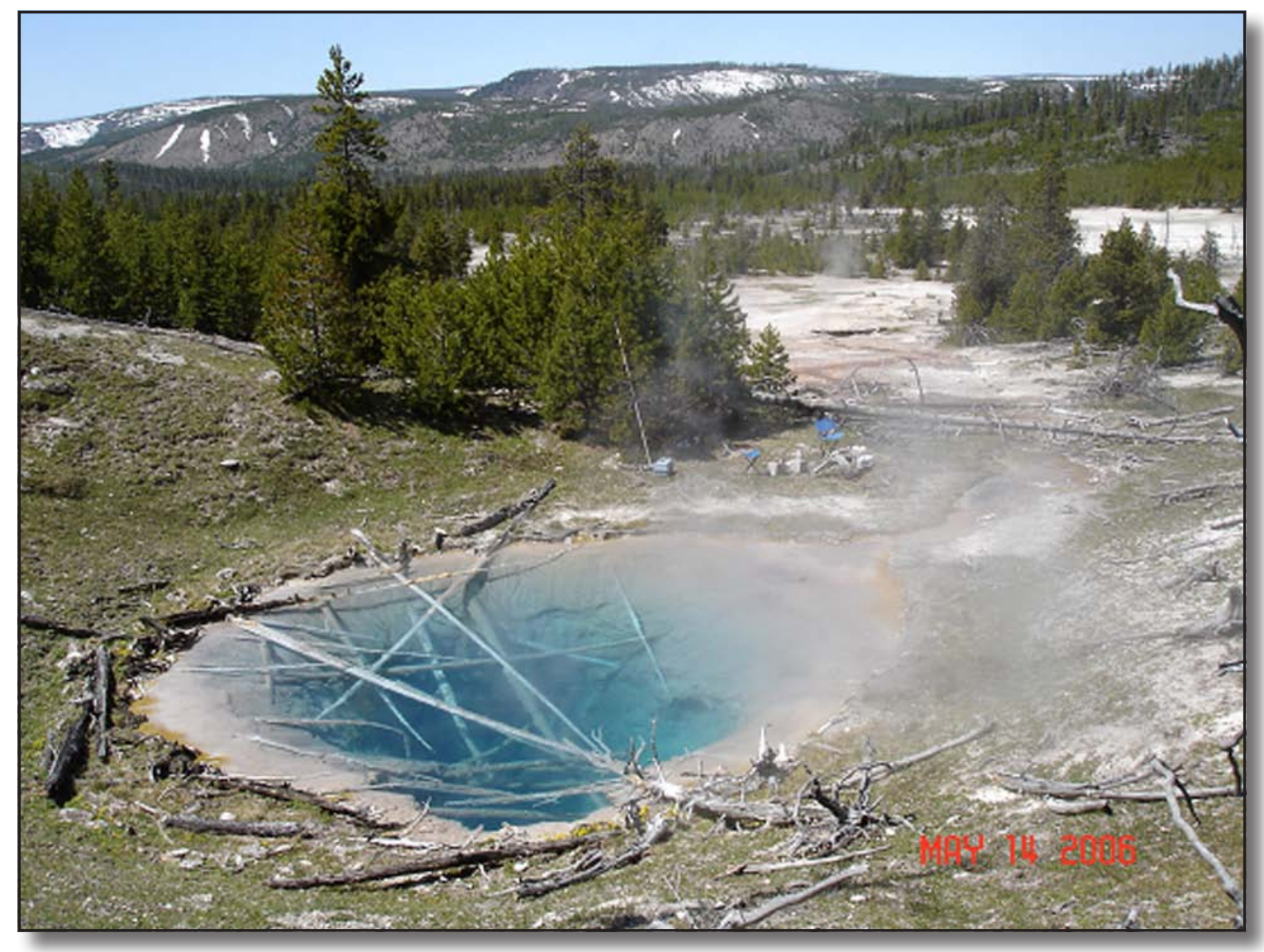

Open-File Report 2010-1192 
Front Cover Photography: View taken in 2006 of an unnamed hot spring in the Rabbit Creek area, Yellowstone National Park.

Back Cover Photography: View taken in 2006 of an unnamed hot spring near Perpetual Spouter, Norris Geyser Basin, Yellowstone National Park 


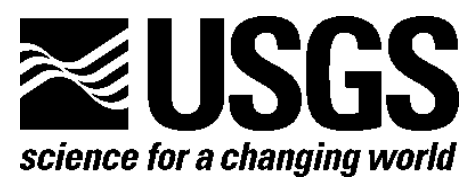

\section{Water-Chemistry Data for Selected Springs, Geysers, and Streams in Yellowstone National Park, Wyoming, 2006-2008}

By James W. Ball, R. Blaine McCleskey, and D. Kirk Nordstrom

Open-File Report 2010-1192

U.S. Department of the Interior

U.S. Geological Survey 


\section{U.S. Department of the Interior \\ KEN SALAZAR, Secretary}

\section{U.S. Geological Survey \\ Marcia K. McNutt, Director}

U.S. Geological Survey, Reston, Virginia 2010

For product and ordering information:

World Wide Web: http://www.usgs.gov/pubprod

Telephone: 1-888-ASK-USGS

For more information on the USGS-the Federal source for science about the Earth, its natural and living resources, natural hazards, and the environment:

World Wide Web: http://www.usgs.gov

Telephone: 1-888-ASK-USGS

Any use of trade, product, or firm names is for descriptive purposes only and does not imply endorsement by the U.S. Government.

Although this report is in the public domain, permission must be secured from the individual copyright owners to reproduce any copyrighted material contained within this report.

Suggested citation:

Ball, J.W., McCleskey, R.B., and Nordstrom, D.K., 2010, Water-chemistry data for selected springs, geysers, and streams in Yellowstone National Park, Wyoming, 2006-2008: U.S. Geological Survey Open-File Report 2010-1192, 109 p. 


\section{Contents}

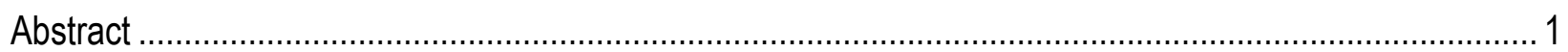

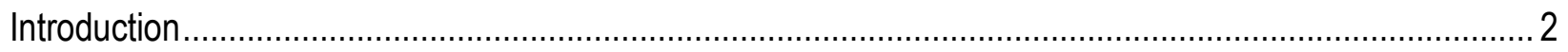

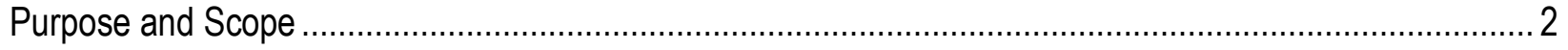

Methods of Sample Collection, Preservation, and Analysis ................................................................ 5

Sample Locations ...........................................................................................................

Sample Collection and Preservation ........................................................................................... 5

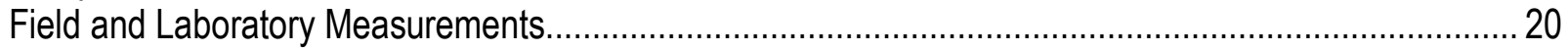

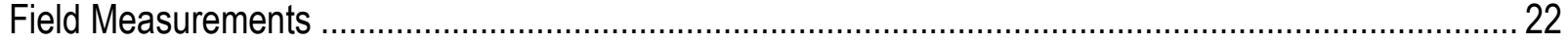

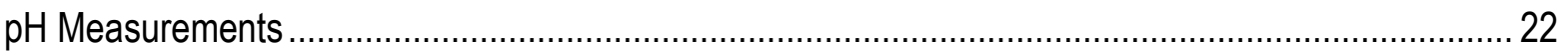

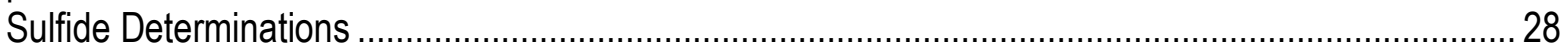

Major Cation and Trace Metal Determinations ............................................................................ 30

Major Anion Determinations ............................................................................................. 30

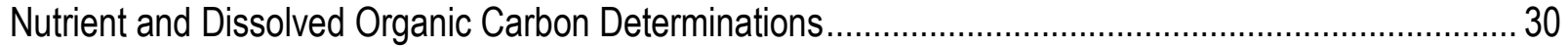

Water Isotope Determinations................................................................................................. 31

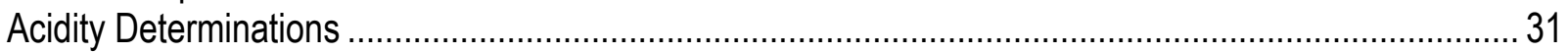

Revised pH Measurements................................................................................................ 33

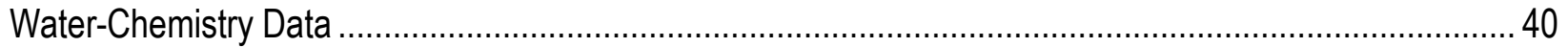

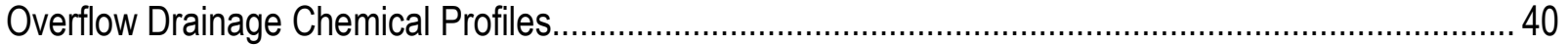

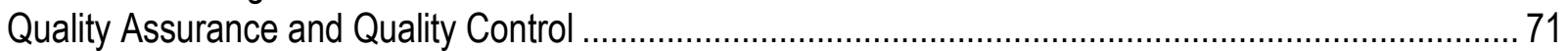

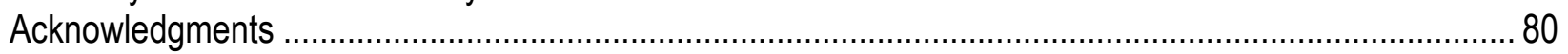

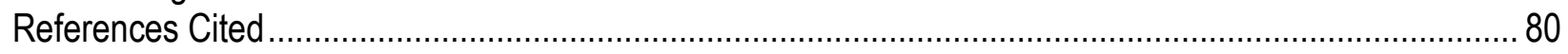

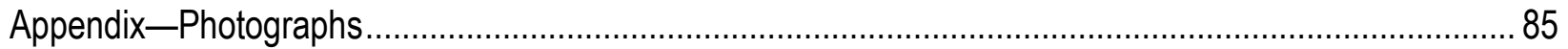

\section{Figures}

1. Location of sampling areas in Yellowstone National Park, Wyoming ......................................... 4

2. Sampling locations for hot springs, geysers, and surface waters at Norris Geyser Basin and West Nymph Creek thermal area, Yellowstone National Park, Wyoming................................... 8

3-13. Sampling locations with sample code numbers for hot springs and two surface-water samples, Yellowstone National Park, Wyoming

3. One Hundred Spring Plain, Norris Geyser Basin .......................................................... 9

4. Back Basin, Norris Geyser Basin ............................................................................. 10

5. Ragged Hills, Geyser Basin .................................................................................... 11

6. West Nymph Creek Thermal Area ......................................................................... 12

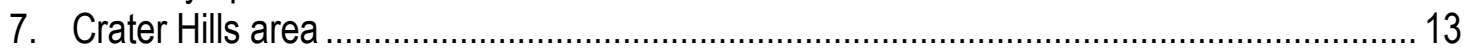

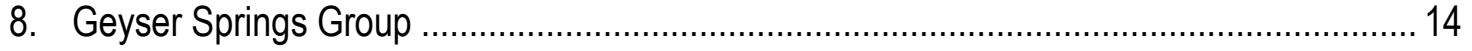

9. Ojo Caliente Spring, Lower Geyser Basin ................................................................. 15

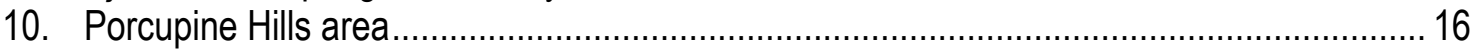

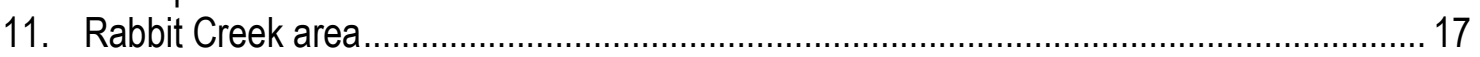

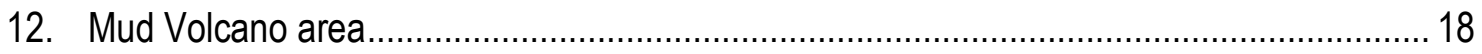

13. Washburn Hot Springs ................................................................................... 19

14. Comparison of results of sulfide determinations using standard and modified methylene - blue colorimetric methods.

15. Comparison of results of sulfide determinations using the ion-selective electrode method and best values from the methylene - blue colorimetric method.

16. Flow chart illustrating the process for refining the acidity $\mathrm{pH}$ value...........................................33 


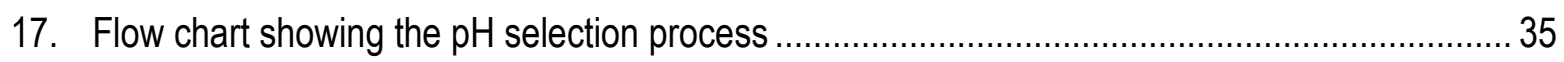

18-20. Difference between field and laboratory $\mathrm{pH}$ as a function of:

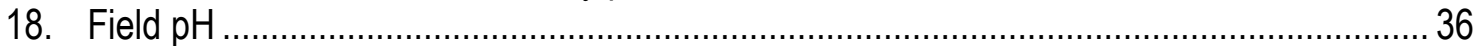

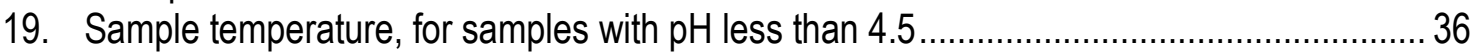

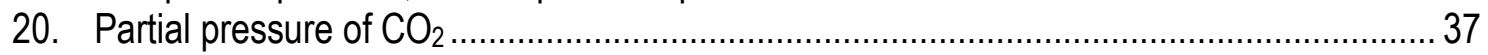

21. Difference between speciated charge imbalance at $22^{\circ} \mathrm{C}$ and speciated charge imbalance at field temperature in relation to field temperature

22. Sample temperature and $\mathrm{pH}(\mathrm{A})$, specific conductance and concentration of calcium (B), concentrations of sulfate and chloride (C), and concentrations of fluoride and aluminum (D) as a function of drainage distance for Succession Spring and its overflow drainage channel taken in 2006

23. Concentrations of mercury and arsenic(total) (A), concentrations of iron (total), iron (II), and iron (III) (B), concentrations of sodium and ammonium (C), and delta deuterium and delta 180 (D) as a function of drainage distance for Succession Spring and its overflow drainage channel taken in 2006 .....

24. Sample temperature and $\mathrm{pH}(\mathrm{A})$, field and laboratory specific conductance $(B)$, concentrations of sulfate and chloride (C), and concentrations of lithium and boron (D) as a function of drainage distance for Ojo Caliente Spring and its overflow drainage channel taken in 2008

25. Concentrations of sodium and potassium (A), concentrations of nitrate and dissolved oxygen (B), and concentration of arsenic (total) (C) as a function of drainage distance for Ojo Caliente Spring and its overflow drainage channel taken in 2008.

26. Sample temperature and $\mathrm{pH}(\mathrm{A})$, specific conductance and concentration of calcium (B), concentrations of sulfate and chloride (C), and concentrations of fluoride and aluminum (D) as a function of drainage distance for an unnamed thermal feature and its overflow drainage channel in the upper Rabbit Creek area taken in 2006

27. Concentrations of sodium and lithium (A), concentrations of boron and antimony (total) (B), concentrations of mercury and arsenic (total) (C), and delta deuterium and delta 180 (D) as a function of drainage distance for an unnamed thermal feature and its overflow drainage channel in the upper Rabbit Creek area taken in 2006

28. Sample temperature and pH (A), specific conductance, and concentration of calcium (B), concentrations of sulfate and chloride (C), and concentrations of fluoride and aluminum (D) as a function of drainage distance for an unnamed thermal feature and its overflow drainage channel in the lower Rabbit Creek area taken in 2006

29. Concentrations of boron and lithium (A), concentrations of sodium and bicarbonate (B), concentrations of mercury and arsenic(total) (C), and delta deuterium and delta 180 (D) as a function of drainage distance for an unnamed thermal feature and its overflow drainage channel in the lower Rabbit Creek area taken in 2006

30. Frequency distribution of charge imbalance for samples having major cation and anion determinations

31. Fluoride (A), total dissolved arsenic $(B)$, and total dissolved iron (C) concentrations determined by alternative methods

\section{Tables}

1. Detailed sample site descriptions, locations, map, photograph, and chemical data table reference

2. Container preparation and stabilization methods for filtered samples. 
3. Analytical techniques, detection limits, typical precision, equipment used, and analytical method references.

4. Sample $\mathrm{pH}$ measured in the field and laboratory and calculated from acidity titration and speciated charge imbalance, for samples with $\mathrm{pH}$ less than 4

5-13. Results of water analyses for:

5. Norris-Mammoth corridor and the West Nymph Creek thermal area, 2006-2008.

6. Norris Geyser Basin, 2006-2008

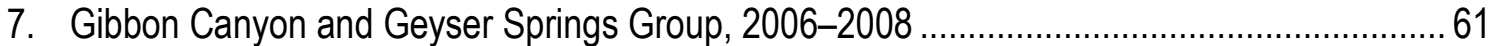

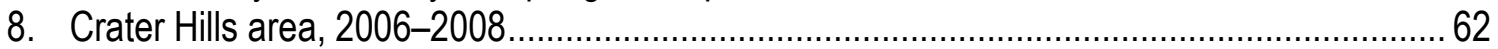

9. Ojo Caliente Spring and its drainage channel, Lower Geyser Basin, 2006-2008 ..................63

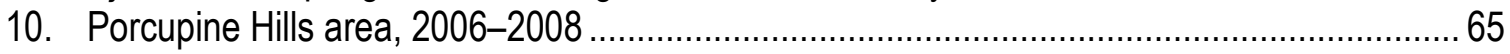

11. Midway Geyser Basin and the Rabbit Creek area, 2006-2008 .........................................67

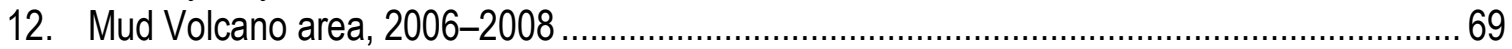

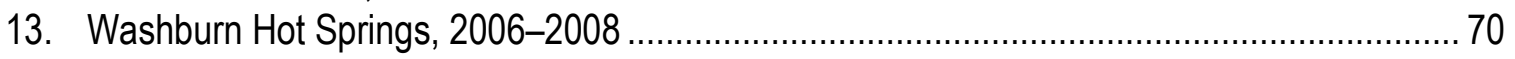

14. Measurement of concentrations of dissolved constituents in standard reference water samples 


\section{Conversion Factors}

SI to Inch/Pound

\begin{tabular}{|c|c|c|}
\hline Multiply & By & To obtain \\
\hline \multicolumn{3}{|c|}{ Length } \\
\hline nanometer (nm) & 0.00000003937 & inch (in.) \\
\hline micrometer $(\mu \mathrm{m})$ & 0.00003937 & inch (in.) \\
\hline millimeter $(\mathrm{mm})$ & 0.03937 & inch (in.) \\
\hline centimeter $(\mathrm{cm})$ & 0.3937 & inch (in.) \\
\hline $\operatorname{meter}(\mathrm{m})$ & 3.281 & foot $(\mathrm{ft})$ \\
\hline kilometer $(\mathrm{km})$ & 0.6214 & mile (mi) \\
\hline kilometer (km) & 0.5400 & mile, nautical (nmi) \\
\hline meter $(\mathrm{m})$ & 1.094 & yard (yd) \\
\hline \multicolumn{3}{|c|}{ Volume } \\
\hline microliter $(\mu \mathrm{L})$ & 0.0000338 & ounce, fluid (fl. oz) \\
\hline milliliter $(\mathrm{mL})$ & 0.0338 & ounce, fluid (fl. oz) \\
\hline liter (L) & 33.82 & ounce, fluid (fl. oz) \\
\hline liter (L) & 2.113 & pint (pt) \\
\hline liter (L) & 1.057 & quart (qt) \\
\hline liter (L) & 0.2642 & gallon (gal) \\
\hline \multicolumn{3}{|c|}{ Mass } \\
\hline nanogram (ng) & 0.00000000003527 & ounce, avoirdupois (oz) \\
\hline microgram $(\mu \mathrm{g})$ & 0.00000003527 & ounce, avoirdupois (oz) \\
\hline milligram (mg) & 0.00003527 & ounce, avoirdupois (oz) \\
\hline $\operatorname{gram}(\mathrm{g})$ & 0.03527 & ounce, avoirdupois (oz) \\
\hline kilogram $(\mathrm{kg})$ & 2.205 & pound avoirdupois (lb) \\
\hline \multicolumn{3}{|c|}{ Density } \\
\hline kilogram per cubic meter $\left(\mathrm{kg} / \mathrm{m}^{3}\right)$ & 0.06242 & pound per cubic foot $\left(\mathrm{lb} / \mathrm{ft}^{3}\right)$ \\
\hline gram per cubic centimeter $\left(\mathrm{g} / \mathrm{cm}^{3}\right)$ & 62.4220 & pound per cubic foot $\left(\mathrm{lb} / \mathrm{ft}^{3}\right)$ \\
\hline
\end{tabular}

Temperature in degrees Celsius $\left({ }^{\circ} \mathrm{C}\right)$ may be converted to degrees Fahrenheit $\left({ }^{\circ} \mathrm{F}\right)$ as follows:

${ }^{\circ} \mathrm{F}=\left(1.8 \times{ }^{\circ} \mathrm{C}\right)+32$

Temperature in degrees Fahrenheit $\left({ }^{\circ} \mathrm{F}\right)$ may be converted to degrees Celsius $\left({ }^{\circ} \mathrm{C}\right)$ as follows:

${ }^{\circ} \mathrm{C}=\left({ }^{\circ} \mathrm{F}-32\right) / 1.8$

Horizontal coordinate information is referenced to the North American Datum of 1927 (NAD 27) 


\title{
Water-Chemistry Data for Selected Springs, Geysers, and Streams in Yellowstone National Park, Wyoming, 2006-2008
}

\author{
By James W. Ball, R. Blaine McCleskey, and D. Kirk Nordstrom
}

\begin{abstract}
Water analyses are reported for 104 samples collected from numerous thermal and nonthermal features in Yellowstone National Park (YNP) during 2006-2008. Water samples were collected and analyzed for major and trace constituents from 10 areas of YNP including Apollinaris Spring and Nymphy Creek along the Norris-Mammoth corridor, Beryl Spring in Gibbon Canyon, Norris Geyser Basin, Lower Geyser Basin, Crater Hills, the Geyser Springs Group, Nez Perce Creek, Rabbit Creek, the Mud Volcano area, and Washburn Hot Springs. These water samples were collected and analyzed as part of research investigations in YNP on arsenic, antimony, iron, nitrogen, and sulfur redox species in hot springs and overflow drainages, and the occurrence and distribution of dissolved mercury. Most samples were analyzed for major cations and anions, trace metals, redox species of antimony, arsenic, iron, nitrogen, and sulfur, and isotopes of hydrogen and oxygen. Analyses were performed at the sampling site, in an onsite mobile laboratory vehicle, or later in a U.S. Geological Survey laboratory, depending on stability of the constituent and whether it could be preserved effectively.

Water samples were filtered and preserved on-site. Water temperature, specific conductance, $\mathrm{pH}$, emf (electromotive force or electrical potential), and dissolved hydrogen sulfide were measured on-site at the time of sampling. Dissolved hydrogen sulfide was measured a few to several hours after sample collection by ion-specific electrode on samples preserved onsite. Acidity was determined by titration, usually within a few days of sample collection. Alkalinity was determined by titration within 1 to 2 weeks of sample collection. Concentrations of thiosulfate and polythionate were determined as soon as possible (generally a few to several hours after sample collection) by ion chromatography in an on-site mobile laboratory vehicle. Total dissolved iron and ferrous iron concentrations often were measured on $\square$ site in the mobile laboratory vehicle.

Concentrations of dissolved aluminum, arsenic, boron, barium, beryllium, calcium, cadmium, cobalt, chromium, copper, iron, potassium, lithium, magnesium, manganese, molybdenum, sodium, nickel, lead, selenium, silica, strontium, vanadium, and zinc were determined by inductively coupled plasma-optical emission spectrometry. Trace concentrations of dissolved antimony, cadmium, cobalt, chromium, copper, lead, and selenium were determined by Zeeman-corrected graphite-furnace atomic-absorption spectrometry. Dissolved concentrations of total arsenic, arsenite, total antimony, and antimonite were determined by hydride generation atomic-absorption spectrometry using a flow-injection analysis system. Dissolved concentrations of total mercury and methylmercury were determined by cold-vapor atomic fluorescence spectrometry. Concentrations of dissolved chloride, fluoride, nitrate, bromide, and sulfate were determined by ion chromatography. For many samples, concentrations of dissolved fluoride also
\end{abstract}


were determined by ion-specific electrode. Concentrations of dissolved ferrous and total iron were determined by the FerroZine colorimetric method. Concentrations of dissolved ammonium were determined by ion chromatography, with reanalysis by colorimetry when separation of sodium and ammonia peaks was poor. Dissolved organic carbon concentrations were determined by the wet persulfate oxidation method. Hydrogen and oxygen isotope ratios were determined using the hydrogen and $\mathrm{CO}_{2}$ equilibration techniques, respectively.

\section{Introduction}

Investigations into the water chemistry of hot springs, geysers, streams, and rivers in Yellowstone National Park (YNP) have been conducted by the U.S. Geological Survey (USGS) and other earth-science organizations and academic institutions dating back to 1888. A list of publications that report results of these investigations is presented in table 1 of Ball and others (2006). Waters at YNP have $\mathrm{pH}$ values ranging from about 1 to 10 , temperatures from ambient to about 95 degrees Celsius $\left({ }^{\circ} \mathrm{C} ; 93^{\circ} \mathrm{C}\right.$ is boiling at YNP's altitude; some pools are superheated), and high concentrations of silica, arsenic, boron, fluoride, hydrogen sulfide, lithium, sulfate, and alkalinity relative to most natural waters. Numerous redox reactions and mineral-precipitation reactions occur. In addition to being valuable natural resources and tourist attractions, active geothermal areas such as those in YNP provide insight into formation of mineral deposits, microbiological processes in extreme environments, and water-rock interactions.

\section{Purpose and Scope}

The purpose of this report is to: (1) provide water-chemistry data from numerous sample sites in YNP for 2006-2008; (2) describe methods used to collect and analyze the samples; (3) describe quality-assurance and quality-control procedures; and (4) supplement interpretive reports. The samples were collected during four field trips to YNP: May and September 2006, September 2007, and September 2008. The primary purpose of the field trips was to study and interpret the geochemical processes (including mineral precipitation) affecting redox species of antimony, arsenic, iron, nitrogen, and sulfur in mineral springs and geothermal waters and their drainages. The evolution and chemical composition of several newly developed hydrothermal features of the Ragged Hills area of Norris Geyser Basin and the occurrence and distribution of total dissolved mercury and methylmercury in geothermal waters also was studied during these field trips. Several papers have been published on earlier phases of this project and of other collaborative research efforts (Xu and Schoonen, 1995; Xu and others, 1996; 1998; 2000; McCleskey and others, 2004; Ball and others, 1998a, b; 2001; 2002; 2006; Nordstrom and others, 2005; King and others, 2006; Planer-Friedrich and others, 2007; Boyd and others, 2009; Nordstrom and others, 2009; Sherman and others, 2009).

During 2006-2008, 104 water samples were collected from YNP (fig. 1). Five individual samples were collected from Apollinaris Spring and Nymphy Creek along the Norris-Mammoth corridor, Beryl Spring near Gibbon Canyon, and Excelsior Geyser Crater at Midway Geyser Basin. The remaining 99 samples were collected from eight areas of YNP: Norris Geyser Basin, Crater Hills, the Geyser Springs Group, Lower Geyser Basin, Porcupine Hills, Rabbit Creek, Mud Volcano, and Washburn Hot Springs. Thirty-five analyses are reported for samples collected for May-June 2006, 20 analyses are reported for September 2006, 21 analyses are reported for September 2007, and 28 analyses are reported for September 2008. Constituents determined include dissolved concentrations of calcium $(\mathrm{Ca})$, magnesium $(\mathrm{Mg})$, strontium $(\mathrm{Sr})$, barium $(\mathrm{Ba})$, sodium $(\mathrm{Na})$, potassium $(\mathrm{K})$, lithium $(\mathrm{Li})$, sulfate $\left(\mathrm{SO}_{4}\right)$, thiosulfate $\left(\mathrm{S}_{2} \mathrm{O}_{3}\right)$, polythionate $\left(\mathrm{S}_{\mathrm{n}} \mathrm{O}_{6}\right)$, hydrogen sulfide $\left(\mathrm{H}_{2} \mathrm{~S}\right)$, alkalinity $\left(\right.$ as $\left.\mathrm{HCO}_{3}\right)$, acidity, fluoride $(\mathrm{F})$, chloride 
$(\mathrm{Cl})$, bromide $(\mathrm{Br})$, nitrate $\left(\mathrm{NO}_{3}\right)$, ammonium $\left(\mathrm{NH}_{4}\right)$, silica $\left(\mathrm{SiO}_{2}\right)$, boron $(\mathrm{B})$, aluminum $(\mathrm{Al})$, total iron $(\mathrm{Fe}(\mathrm{T}))$, ferrous iron $(\mathrm{Fe}(\mathrm{II}))$, manganese $(\mathrm{Mn})$, copper $(\mathrm{Cu})$, zinc $(\mathrm{Zn})$, cadmium $(\mathrm{Cd})$, chromium $(\mathrm{Cr})$, cobalt $(\mathrm{Co})$, total mercury $(\mathrm{Hg}(\mathrm{T}))$, methylmercury $\left(\mathrm{CH}_{3} \mathrm{Hg}\right)$, nickel $(\mathrm{Ni})$, lead $(\mathrm{Pb})$, beryllium $(\mathrm{Be})$, vanadium $(\mathrm{V})$, molybdenum $(\mathrm{Mo})$, total antimony $(\mathrm{Sb}(\mathrm{T}))$, antimonite $(\mathrm{Sb}(\mathrm{III})$ ), selenium (Se), total arsenic $(\mathrm{As}(\mathrm{T}))$, arsenite $(\mathrm{As}(\mathrm{III}))$, dissolved organic carbon $(\mathrm{DOC})$, and isotopes of hydrogen $(\delta \mathrm{D})$ and oxygen $\left(\delta^{18} \mathrm{O}\right)$. 

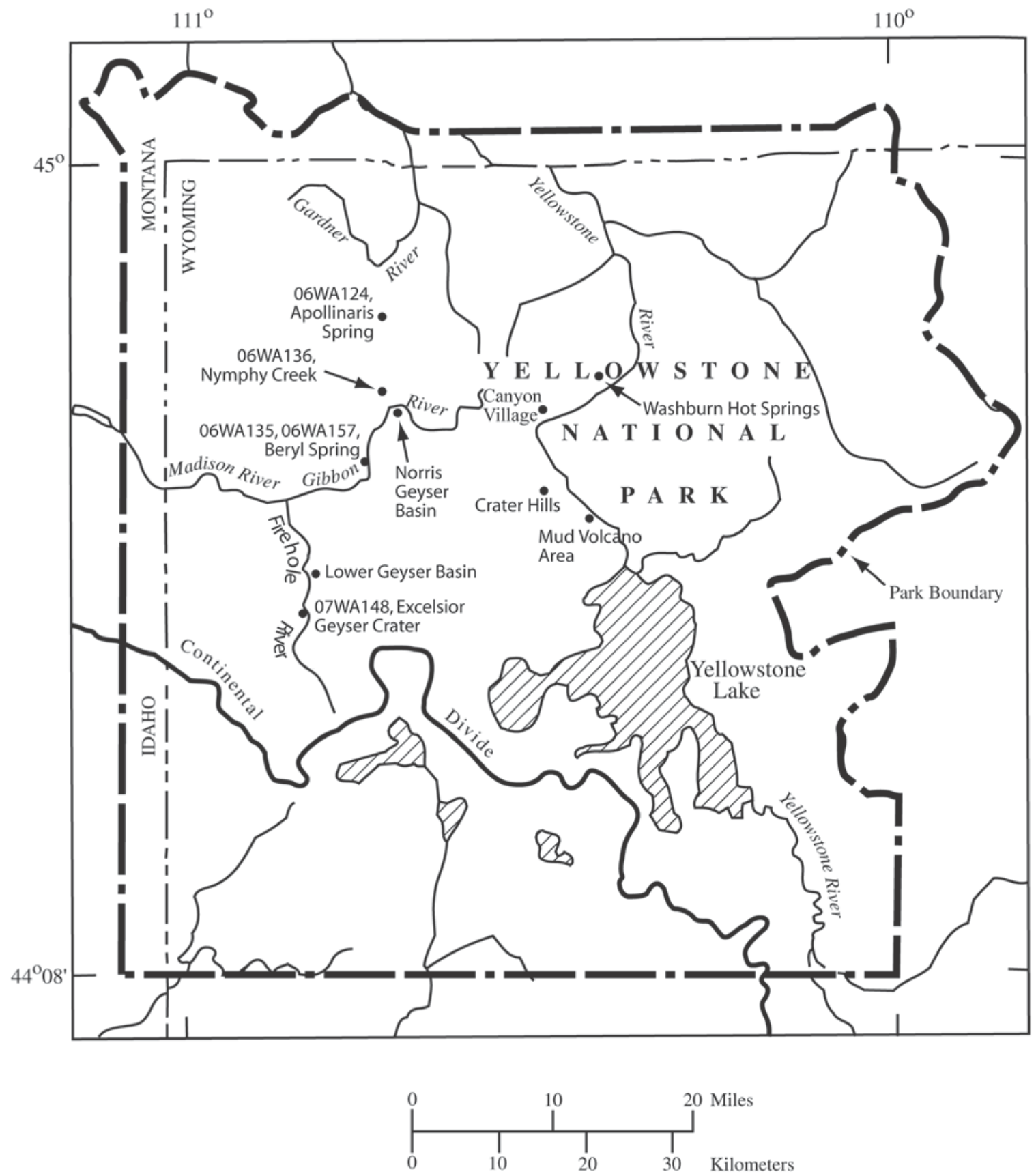

Base from Stauffer and others (1980)

Figure 1. Location of sampling areas in Yellowstone National Park, Wyoming. 


\section{Methods of Sample Collection, Preservation, and Analysis}

\section{Sample Locations}

Because many geothermal features can be within meters of each other, it is critical that sample sites be accurately located and described. To this end, we have made every effort to include detailed maps (figs. 2-12), written descriptions and accurate latitude and longitude measurements (table 1), and photographs (Appendix) of all the sample sites for which analytical results are reported.

The locations of sampling areas in YNP, and for Apollinaris and Beryl Springs, Excelsior Geyser Crater, and Nymphy Creek, are shown on figure 1. Sample locations for the remaining eight areas sampled are shown on the following maps: Norris Geyser Basin (figs. 2-5), West Nymph Creek Thermal Area (fig. 6), Crater Hills (fig. 7), the Geyser Springs Group (fig. 8), Lower Geyser Basin (fig. 9), the Porcupine Hills area (fig. 10), the Rabbit Creek thermal area (fig. 11), the Mud Volcano area (fig. 12) and Washburn Hot Springs (fig. 13). Official names are taken from either the Geographic Names Information System (GNIS; http://geonames.usgs.gov/) or Whittlesey (1988). Several features that did not have official names were assigned names by the authors for project purposes. Features named by the authors are in quotations in tables 1 and $5-14$.

For terminology in this report, "source" samples were collected at the origin of the spring, and overflow "drainage channel" samples were collected at various distances downstream from the source. Series of transect samples were collected in the overflow drainage channels from Ojo Caliente Spring, "Succession Spring" in the Ragged Hills area of Norris Geyser Basin, and drainage channels from two unnamed hot springs in the Rabbit Creek area. Latitudes and longitudes were acquired using a portable global positioning system (GPS; Garmin III+ or Garmin Rino 530) with latitudes and longitudes referenced to the NAD27 datum (North American Datum of 1927), compatible with USGS topographic maps.

\section{Sample Collection and Preservation}

Samples were collected from streams, tributaries, and overflow drainage channels as close to the center of flow as possible and in areas that appeared to be well mixed. For springs and geysers, samples were collected as close to the discharge source as possible. Extreme care was taken to safely collect water samples from the geothermal sites, to protect fragile hot-spring mineral formations, and to minimize changes in temperature, $\mathrm{pH}$, and water chemistry during sampling. Samples were collected from the middle of large pools and geysers by positioning the sample tubing intake using an insulated stainless-steel container as a flotation device attached to the end of an extendable aluminum pole. At more easily accessible sites, the tubing intake was positioned in the source or channel by hand. A Teflon block attached to the end of the sampling tubing was used as a weight to keep the sample tubing in place (McCleskey and others, 2004).

Samples were collected and filtered on $\square$ site by either or both of two techniques. The first technique consisted of pumping the water directly from the source with a battery-operated peristaltic pump fitted with medical-grade silicone tubing through a pre-cleaned 142-millimeter (mm) diameter all-plastic filter holder (Kennedy and others, 1976) containing a 0.1-micrometer $(\mu \mathrm{m})$ pore size mixed-cellulose-ester filter membrane. The second technique consisted of filling a 60 -milliliter $(\mathrm{mL})$ syringe by suctioning from the source, rinsing three times, and immediately forcing the water through a $25-\mathrm{mm}$ disposable filter having a mixed-cellulose-ester membrane with a pore size of 0.2 or $0.45 \mu \mathrm{m}$. 
Table 1. Detailed sample site descriptions, locations, map, photograph, and chemical data table reference.

[--, not measured or not photographed; cm, centimeters; m, meters]

\begin{tabular}{|c|c|c|c|c|c|c|}
\hline Name and Site Description & $\begin{array}{c}\text { Sampl e } \\
\text { code } \\
\text { number }\end{array}$ & $\begin{array}{c}\text { North } \\
\text { Lat i t ude }\end{array}$ & $\begin{array}{c}\text { West } \\
\text { Longit ude }\end{array}$ & $\begin{array}{c}\text { Chemi cal } \\
\text { dat a } \\
(\mathrm{t} \text { abl e })\end{array}$ & $\begin{array}{c}\text { Map } \\
(\mathrm{f} \text { i gure) }\end{array}$ & $\begin{array}{l}\text { Phot ograph } \\
\text { (appendix) }\end{array}$ \\
\hline \multicolumn{7}{|l|}{ Norris-Mammoth Corridor } \\
\hline Apollinaris Spring & 06WA124 & $44^{\circ} 50^{\prime} 34.0^{\prime \prime}$ & $110^{\circ} 43^{\prime} 57.8^{\prime \prime}$ & 5 & 1 & 24 \\
\hline Nymphy Creek & 06WA136 & $44^{\circ} 45^{\prime} 12.5^{\prime \prime}$ & $110^{\circ} 43^{\prime} 33.5^{\prime \prime}$ & 5 & 1 & 35 \\
\hline \multicolumn{7}{|l|}{ West Nymph Creek Thermal Area } \\
\hline Uppermost pool, West Nymph Creek T hermal Area & 08WA118 & $44^{\circ} 44^{\prime} 44.4^{\prime \prime}$ & $110^{\circ} 44^{\prime} 40.4^{\prime \prime}$ & 5 & 6 & 80 \\
\hline Sunken circular pool, West Nymph Creek Thermal Area & 08WA119 & $44^{\circ} 44^{\prime} 34.6^{\prime \prime}$ & $110^{\circ} 44^{\prime} 40.5^{\prime \prime}$ & 5 & 6 & 81 \\
\hline \multicolumn{7}{|l|}{ Norris Geyser Basin (One Hundred Spring P lain area) } \\
\hline Cinder Pool & 07WA113 & $44^{\circ} 43^{\prime} 57.2^{\prime \prime}$ & $110^{\circ} 42^{\prime} 35.6^{\prime \prime}$ & 6 & 3 & 61 \\
\hline Realgar Creek at mouth & 06WA106 & $44^{\circ} 44^{\prime} 16.8^{\prime \prime}$ & $110^{\circ} 42^{\prime} 32.7^{\prime \prime}$ & 6 & 3 & 1 \\
\hline Realgar Creek at mouth & 06WA139 & $44^{\circ} 44^{\prime} 16.8^{\prime \prime}$ & $110^{\circ} 42^{\prime} 32.7^{\prime \prime}$ & 6 & 3 & 36 \\
\hline Tantalus Creek at weir & 06WA107 & $44^{\circ} 44^{\prime} 2.7^{\prime \prime}$ & $110^{\circ} 42^{\prime} 54.6^{\prime \prime}$ & 6 & 3 & 2 \\
\hline Tantalus Creek at weir & 06WA158 & $44^{\circ} 44^{\prime} 2.7^{\prime \prime}$ & $110^{\circ} 42^{\prime} 54.6^{\prime \prime}$ & 6 & 3 & 39 \\
\hline Tantalus Creek at weir & 08WA105 & $44^{\circ} 44^{\prime} 2.7^{\prime \prime}$ & $110^{\circ} 42^{\prime} 54.6^{\prime \prime}$ & 6 & 3 & 73 \\
\hline Unnamed hot spring northeast of Cinder Pool & 06WA109 & $44^{\circ} 43^{\prime} 59.1^{\prime \prime}$ & $110^{\circ} 42^{\prime} 29.3^{\prime \prime}$ & 6 & 3 & 4 \\
\hline \multicolumn{7}{|l|}{ Norris Geyser Basin (Back Basin area) } \\
\hline Cistern Spring & 06WA123 & $44^{\circ} 43^{\prime} 23.4^{\prime \prime}$ & $110^{\circ} 42^{\prime} 14.8^{\prime \prime}$ & 6 & 4 & 23 \\
\hline Cistern Spring & 06WA159 & $44^{\circ} 43^{\prime} 23.4^{\prime \prime}$ & $110^{\circ} 42^{\prime} 14.8^{\prime \prime}$ & 6 & 4 & 40 \\
\hline Cistern Spring & 07WA116 & $44^{\circ} 43^{\prime} 23.4^{\prime \prime}$ & $110^{\circ} 42^{\prime} 14.8^{\prime \prime}$ & 6 & 4 & 64 \\
\hline Cistern Spring & 08WA127 & $44^{\circ} 43^{\prime} 23.4^{\prime \prime}$ & $110^{\circ} 42^{\prime} 14.8^{\prime \prime}$ & 6 & 4 & 89 \\
\hline Echinus Geyser & 08WA126 & $44^{\circ} 43^{\prime} 20.0^{\prime \prime}$ & $110^{\circ} 42^{\prime} 4.8^{\prime \prime}$ & 6 & 4 & 88 \\
\hline Perpetual Spouter & 06WA132 & $44^{\circ} 43^{\prime} 35.7^{\prime \prime}$ & $110^{\circ} 42^{\prime} 32.7^{\prime \prime}$ & 6 & 4 & 31 \\
\hline Perpetual Spouter & 07WA144 & $44^{\circ} 43^{\prime} 35.7^{\prime \prime}$ & $110^{\circ} 42^{\prime} 32.7^{\prime \prime}$ & 6 & 4 & 69 \\
\hline Perpetual Spouter & 08WA130 & $44^{\circ} 43^{\prime} 35.7^{\prime \prime}$ & $110^{\circ} 42^{\prime} 32.7^{\prime \prime}$ & 6 & 4 & 92 \\
\hline Porkchop Geyser & 07WA143 & $44^{\circ} 43^{\prime} 20.0^{\prime \prime}$ & $110^{\circ} 42^{\prime} 26.6^{\prime \prime}$ & 6 & 4 & 68 \\
\hline Porkchop Geyser & 08WA129 & $44^{\circ} 43^{\prime} 20.0^{\prime \prime}$ & $110^{\circ} 42^{\prime} 26.6^{\prime \prime}$ & 6 & 4 & 91 \\
\hline Second Erupter & 06WA122 & $44^{\circ} 43^{\prime} 20.2^{\prime \prime}$ & $110^{\circ} 42^{\prime} 24.5^{\prime \prime}$ & 6 & 4 & 22 \\
\hline Second Erupter & 07WA142 & $44^{\circ} 43^{\prime} 20.2^{\prime \prime}$ & $110^{\circ} 42^{\prime} 24.5^{\prime \prime}$ & 6 & 4 & 67 \\
\hline Second Erupter & 08WA128 & $44^{\circ} 43^{\prime} 20.3^{\prime \prime}$ & $110^{\circ} 42^{\prime} 24.2^{\prime \prime}$ & 6 & 4 & 90 \\
\hline Tantalus Creek - Back Basin Drainage & 06WA134 & $44^{\circ} 43^{\prime} 35.4^{\prime \prime}$ & $110^{\circ} 42^{\prime} 29.9^{\prime \prime}$ & 6 & 4 & 33 \\
\hline Unnamed acid spring next To Perpetual Spouter & 06WA133 & $44^{\circ} 43^{\prime} 35.5^{\prime \prime}$ & $110^{\circ} 42^{\prime} 33.1^{\prime \prime}$ & 6 & 4 & 32 \\
\hline Unnamed acid spring next To Perpetual Spouter & 07WA145 & $44^{\circ} 43^{\prime} 35.5^{\prime \prime}$ & $110^{\circ} 42^{\prime} 33.1^{\prime \prime}$ & 6 & 4 & 70 \\
\hline Unnamed hot spring, north end of Elk Park & 07WA140 & $44^{\circ} 43^{\prime} 11.7^{\prime \prime}$ & $110^{\circ} 42^{\prime} 56.7^{\prime \prime}$ & 6 & 2 & 65 \\
\hline Unnamed hot spring, north end of Elk Park near 07WA140 & 07WA141 & $44^{\circ} 43^{\prime} 11.7^{\prime \prime}$ & $110^{\circ} 42^{\prime} 53.4^{\prime \prime}$ & 6 & 2 & 66 \\
\hline \multicolumn{7}{|l|}{ Norris Geyser Basin (Ragged Hills area) } \\
\hline Appendage side spring to "Lifeboat Spring" & 08WA124 & $44^{\circ} 43^{\prime} 39.8^{\prime \prime}$ & $110^{\circ} 42^{\prime} 49.8^{\prime \prime}$ & 6 & 5 & 86 \\
\hline Circular clay hot spring 2 meters from $08 \mathrm{WA} 121$ & 08WA123 & $44^{\circ} 43^{\prime} 44.3^{\prime \prime}$ & $110^{\circ} 42^{\prime} 41.5^{\prime \prime}$ & 6 & 5 & 85 \\
\hline Crystal Spring - eastern side & 06WA112 & $44^{\circ} 43^{\prime} 43.6^{\prime \prime}$ & $110^{\circ} 42^{\prime} 40.1^{\prime \prime}$ & 6 & 5 & 6 \\
\hline Crystal Spring - eastern side & 07WA147 & $44^{\circ} 43^{\prime} 43.6^{\prime \prime}$ & $110^{\circ} 42^{\prime} 40.1^{\prime \prime}$ & 6 & 5 & 71 \\
\hline Crystal Spring - western side & 06WA111 & $44^{\circ} 43^{\prime} 43.7^{\prime \prime}$ & $110^{\circ} 42^{\prime} 40.2^{\prime \prime}$ & 6 & 5 & 6 \\
\hline Crystal Spring - western side & 07WA146 & $44^{\circ} 43^{\prime} 43.7^{\prime \prime}$ & $110^{\circ} 42^{\prime} 40.2^{\prime \prime}$ & 6 & 5 & 71 \\
\hline Crystal Spring - western side & 08WA120 & $44^{\circ} 43^{\prime} 43.7^{\prime \prime}$ & $110^{\circ} 42^{\prime} 40.2^{\prime \prime}$ & 6 & 5 & 82 \\
\hline The Gap northeast outflow & 06WA121 & $44^{\circ} 43^{\prime} 45.2^{\prime \prime}$ & $110^{\circ} 42^{\prime} 40.5^{\prime \prime}$ & 6 & 5 & 21 \\
\hline The Gap southwest outflow & 06WA120 & $44^{\circ} 43^{\prime} 37.0^{\prime \prime}$ & $110^{\circ} 43^{\prime} 6.0^{\prime \prime}$ & 6 & 2 & 20 \\
\hline Hot Spring next to "Orpiment Puddle 2" & 07WA107 & $44^{\circ} 43^{\prime} 44.3^{\prime \prime}$ & $110^{\circ} 42^{\prime} 41.6^{\prime \prime}$ & 6 & 5 & 55 \\
\hline "Kaolin Spring" & 07WA106 & $44^{\circ} 43^{\prime} 41.8^{\prime \prime}$ & $110^{\circ} 42^{\prime} 44.7^{\prime \prime}$ & 6 & 5 & 54 \\
\hline "Kaolin Spring" & 08WA131 & $44^{\circ} 43^{\prime} 41.8^{\prime \prime}$ & $110^{\circ} 42^{\prime} 44.7^{\prime \prime}$ & 6 & 5 & 93 \\
\hline "Lifeboat Spring" & 06WA118 & $44^{\circ} 43^{\prime} 39.5^{\prime \prime}$ & $110^{\circ} 42^{\prime} 48.2^{\prime \prime}$ & 6 & 5 & 18 \\
\hline "Lifeboat Spring" & 07WA115 & $44^{\circ} 43^{\prime} 39.5^{\prime \prime}$ & $110^{\circ} 42^{\prime} 48.2^{\prime \prime}$ & 6 & 5 & 63 \\
\hline "Lifeboat Spring" & 08WA125 & $44^{\circ} 43^{\prime} 39.5^{\prime \prime}$ & $110^{\circ} 42^{\prime} 48.2^{\prime \prime}$ & 6 & 5 & 87 \\
\hline "Orpiment Puddle 3" & 06WA110 & $44^{\circ} 43^{\prime} 44.4^{\prime \prime}$ & $110^{\circ} 42^{\prime} 41.6^{\prime \prime}$ & 6 & 5 & 5 \\
\hline "Persnickety Geyser" & 06WA119 & $44^{\circ} 43^{\prime} 40.1^{\prime \prime}$ & $110^{\circ} 42^{\prime} 49.9^{\prime \prime}$ & 6 & 5 & 19 \\
\hline "Persnickety Geyser" & 07WA105 & $44^{\circ} 43^{\prime} 40.1^{\prime \prime}$ & $110^{\circ} 42^{\prime} 49.9^{\prime \prime}$ & 6 & 5 & 53 \\
\hline "Succession Spring" & 06WA126 & $44^{\circ} 43^{\prime} 45.8^{\prime \prime}$ & $110^{\circ} 42^{\prime} 41.9^{\prime \prime}$ & 6 & 5 & 26 \\
\hline "Succession Spring" drainage channel & 06WA127 & $44^{\circ} 43^{\prime} 45.8^{\prime \prime}$ & $110^{\circ} 42^{\prime} 41.8^{\prime \prime}$ & 6 & 5 & 27 \\
\hline "Succession Spring" drainage channel & 06WA128 & $44^{\circ} 43^{\prime} 46.0^{\prime \prime}$ & $110^{\circ} 42^{\prime} 41.6^{\prime \prime}$ & 6 & 5 & 28 \\
\hline "Succession Spring" drainage channel & 06WA129 & $44^{\circ} 43^{\prime} 46.0^{\prime \prime}$ & $110^{\circ} 42^{\prime} 41.5^{\prime \prime}$ & 6 & 5 & 29 \\
\hline "Succession Spring" drainage channel & 06WA130 & $44^{\circ} 43^{\prime} 46.1^{\prime \prime}$ & $110^{\circ} 42^{\prime} 41.4^{\prime \prime}$ & 6 & 5 & 29 \\
\hline "Succession Spring" mat & 06WA131 & $44^{\circ} 43^{\prime} 46.2^{\prime \prime}$ & $110^{\circ} 42^{\prime} 41.2^{\prime \prime}$ & 6 & 5 & 30 \\
\hline "Titanic Spring" & 06WA117 & $44^{\circ} 43^{\prime} 39.4^{\prime \prime}$ & $110^{\circ} 42^{\prime} 50.9^{\prime \prime}$ & 6 & 5 & 17 \\
\hline "Titanic Spring" & 07WA114 & $44^{\circ} 43^{\prime} 39.4^{\prime \prime}$ & $110^{\circ} 42^{\prime} 50.9^{\prime \prime}$ & 6 & 5 & 62 \\
\hline Unnamed pool near The Gap & 08WA121 & $44^{\circ} 43^{\prime} 44.3^{\prime \prime}$ & $110^{\circ} 42^{\prime} 41.5^{\prime \prime}$ & 6 & 5 & 83 \\
\hline Unnamed pool near "Succession Spring" & 06WA125 & $44^{\circ} 43^{\prime} 44.3^{\prime \prime}$ & $110^{\circ} 42^{\prime} 44.5^{\prime \prime}$ & 6 & 5 & 25 \\
\hline
\end{tabular}


Table 1. Detailed sample site descriptions, locations, map, photograph, and chemical data table reference-Continued.

\begin{tabular}{|c|c|c|c|c|c|c|}
\hline Name and Site Description & $\begin{array}{c}\text { Sample } \\
\text { code } \\
\text { number }\end{array}$ & $\begin{array}{c}\text { Nort h } \\
\text { Lat i t ude }\end{array}$ & $\begin{array}{c}\text { West } \\
\text { Longi t ude }\end{array}$ & $\begin{array}{c}\text { Chemi cal } \\
\text { dat a } \\
(\mathrm{t} a b \mid \mathrm{e})\end{array}$ & $\begin{array}{c}\text { Map } \\
\text { ( f i gur e) }\end{array}$ & $\begin{array}{l}\text { Photograph } \\
\text { ( appendix) }\end{array}$ \\
\hline \multicolumn{7}{|l|}{ Gibbon Canyon and Geyser Springs Group } \\
\hline Beryl Spring & 06WA135 & $44^{\circ} 40^{\prime} 43.5^{\prime \prime}$ & $110^{\circ} 44^{\prime} 44.7^{\prime \prime}$ & 7 & 1 & 38 \\
\hline Beryl Spring & 06WA157 & $44^{\circ} 40^{\prime} 43.5^{\prime \prime}$ & $110^{\circ} 44^{\prime} 44.7^{\prime \prime}$ & 7 & 1 & 38 \\
\hline Unnamed pool, Geyser Springs Group & 07WA108 & $44^{\circ} 41^{\prime} 25.8^{\prime \prime}$ & $110^{\circ} 43^{\prime} 43.6^{\prime \prime}$ & 7 & 8 & 56 \\
\hline Unnamed pool, Geyser Springs Group & 08WA122 & $44^{\circ} 41^{\prime} 25.8^{\prime \prime}$ & $110^{\circ} 43^{\prime} 43.6^{\prime \prime}$ & 7 & 8 & 84 \\
\hline Unnamed acid pool, Geyser Springs Group & 08WA117 & $44^{\circ} 41^{\prime} 25.3^{\prime \prime}$ & $110^{\circ} 43^{\prime} 42.2^{\prime \prime}$ & 7 & 8 & -- \\
\hline \multicolumn{7}{|l|}{ Crater Hills area } \\
\hline Sulphur Spring & 07WA111 & $44^{\circ} 39^{\prime} 16.7^{\prime \prime}$ & $110^{\circ} 28^{\prime} 54.8^{\prime \prime}$ & 8 & 7 & 59 \\
\hline Sulphur Spring & 08WA115 & $44^{\circ} 39^{\prime} 16.7^{\prime \prime}$ & $110^{\circ} 28^{\prime} 54.8^{\prime \prime}$ & 8 & 7 & 78 \\
\hline Unnamed hot spring & 07WA112 & $44^{\circ} 39^{\prime} 12.1^{\prime \prime}$ & $110^{\circ} 28^{\prime} 36.7^{\prime \prime}$ & 8 & 7 & 60 \\
\hline Unnamed acid pool near Sulphur Spring & 08WA116 & $44^{\circ} 39^{\prime} 15.3^{\prime \prime}$ & $110^{\circ} 28^{\prime} 59.0^{\prime \prime}$ & 8 & 7 & 79 \\
\hline \multicolumn{7}{|l|}{ Lower Geyser Basin } \\
\hline Ojo Caliente Spring at discharge & 06WA160 & $44^{\circ} 33^{\prime} 46.1^{\prime \prime}$ & $110^{\circ} 50^{\prime} 16.9^{\prime \prime}$ & 9 & 9 & -- \\
\hline Ojo Caliente Spring at pool exit & 06WA161 & $44^{\circ} 33^{\prime} 46.5^{\prime \prime}$ & $110^{\circ} 50^{\prime} 17.1^{\prime \prime}$ & 9 & 9 & -- \\
\hline Ojo Caliente Spring $7.5 \mathrm{~m}$ from pool outflow & 06WA162 & $44^{\circ} 33^{\prime} 46.4^{\prime \prime}$ & $110^{\circ} 50^{\prime} 17.2^{\prime \prime}$ & 9 & 9 & -- \\
\hline Ojo Caliente Spring $20.6 \mathrm{~m}$ from pool outflow & 06WA163 & $44^{\circ} 33^{\prime} 46.1^{\prime \prime}$ & $110^{\circ} 50^{\prime} 17.2^{\prime \prime}$ & 9 & 9 & -- \\
\hline Ojo Caliente Spring source, 50 feet below surface & 08WA112 & $44^{\circ} 33^{\prime} 46.1^{\prime \prime}$ & $110^{\circ} 50^{\prime} 16.9^{\prime \prime}$ & 9 & 9 & 76 \\
\hline Ojo Caliente Spring midpoint of main pool & 08WA113 & $44^{\circ} 33^{\prime} 46.3^{\prime \prime}$ & $110^{\circ} 50^{\prime} 17.0^{\prime \prime}$ & 9 & 9 & -- \\
\hline Ojo Caliente Spring, at pool exit & 08WA111 & $44^{\circ} 33^{\prime} 46.5^{\prime \prime}$ & $110^{\circ} 50^{\prime} 17.1^{\prime \prime}$ & 9 & 9 & -- \\
\hline Ojo Caliente Spring $13.8 \mathrm{~m}$ downstream from pool exit & 08WA110 & $44^{\circ} 33^{\prime} 46.3^{\prime \prime}$ & $110^{\circ} 50^{\prime} 17.2^{\prime \prime}$ & 9 & 9 & -- \\
\hline Ojo Caliente Spring $20.4 \mathrm{~m}$ downstream from pool exit & 08WA109 & $44^{\circ} 33^{\prime} 46.1^{\prime \prime}$ & $110^{\circ} 50^{\prime} 17.2^{\prime \prime}$ & 9 & 9 & -- \\
\hline Ojo Caliente Spring $25.9 \mathrm{~m}$ downstream from pool exit & 08WA108 & $44^{\circ} 33^{\prime} 46.0^{\prime \prime}$ & $110^{\circ} 50^{\prime} 17.2^{\prime \prime}$ & 9 & 9 & -- \\
\hline \multicolumn{7}{|l|}{ Porcupine Hills area } \\
\hline Snort Geyser & 06WA173 & $44^{\circ} 34^{\prime} 4.0^{\prime \prime}$ & $110^{\circ} 48^{\prime} 18.4^{\prime \prime}$ & 10 & 10 & 49,50 \\
\hline Unnamed hot spring, Morning Mist Group & 06WA170 & $44^{\circ} 34^{\prime} 26.0^{\prime \prime}$ & $110^{\circ} 48^{\prime} 26.0^{\prime \prime}$ & 10 & 10 & 46 \\
\hline Unnamed hot spring, Quagmire Group & 06WA171 & $44^{\circ} 34^{\prime} 3.7^{\prime \prime}$ & $110^{\circ} 48^{\prime} 26.0^{\prime \prime}$ & 10 & 10 & 47 \\
\hline Unnamed hot spring, Quagmire Group & 06WA172 & $44^{\circ} 34^{\prime} 2.7^{\prime \prime}$ & $110^{\circ} 48^{\prime} 25.0^{\prime \prime}$ & 10 & 10 & 48 \\
\hline Unnamed hot spring, Quagmire Group & 06WA174 & $44^{\circ} 34^{\prime} 1.7^{\prime \prime}$ & $110^{\circ} 48^{\prime} 17.0^{\prime \prime}$ & 10 & 10 & 51 \\
\hline Unnamed hot spring, Quagmire Group & 08WA132 & $44^{\circ} 34^{\prime} 3.7^{\prime \prime}$ & $110^{\circ} 48^{\prime} 26.0^{\prime \prime}$ & 10 & 10 & 94 \\
\hline Unnamed hot spring, Quagmire Group & 08WA133 & $44^{\circ} 34^{\prime} 2.7^{\prime \prime}$ & $110^{\circ} 48^{\prime} 25.0^{\prime \prime}$ & 10 & 10 & 95 \\
\hline Unnamed hot spring, Quagmire Group & 08WA134 & $44^{\circ} 34^{\prime} 1.7^{\prime \prime}$ & $110^{\circ} 48^{\prime} 17.0^{\prime \prime}$ & 10 & 10 & 96 \\
\hline \multicolumn{7}{|l|}{ Midway Geyser Basin and Rabbit Creek area } \\
\hline Excelsior Geyser Crater & 07WA148 & $44^{\circ} 31^{\prime} 34.2^{\prime \prime}$ & $110^{\circ} 50^{\prime} 8.9^{\prime \prime}$ & 11 & 1 & 72 \\
\hline Unnamed hot spring & 06WA113 & $44^{\circ} 31^{\prime} 9.8^{\prime \prime}$ & $110^{\circ} 48^{\prime} 35.3^{\prime \prime}$ & 11 & 11 & 7 \\
\hline Unnamed hot spring drainage channel & 06WA114 & $44^{\circ} 31^{\prime} 9.0^{\prime \prime}$ & $110^{\circ} 48^{\prime} 30.2^{\prime \prime}$ & 11 & 11 & 8 \\
\hline Unnamed hot spring drainage channel & 06WA114B & $44^{\circ} 31^{\prime} 9.0^{\prime \prime}$ & $110^{\circ} 48^{\prime} 30.7^{\prime \prime}$ & 11 & 11 & 9 \\
\hline Unnamed hot spring drainage channel & 06WA114C & $44^{\circ} 31^{\prime} 9.0^{\prime \prime}$ & $110^{\circ} 48^{\prime} 31.8^{\prime \prime}$ & 11 & 11 & 10 \\
\hline Unnamed hot spring drainage channel & 06WA114D & $44^{\circ} 31^{\prime} 9.1^{\prime \prime}$ & $110^{\circ} 48^{\prime} 33.5^{\prime \prime}$ & 11 & 11 & 11 \\
\hline Unnamed hot spring & 06WA115 & $44^{\circ} 31^{\prime} 1.5^{\prime \prime}$ & $110^{\circ} 48^{\prime} 57.2^{\prime \prime}$ & 11 & 11 & 12 \\
\hline Unnamed hot spring & 06WA116 & $44^{\circ} 31^{\prime} 2.1^{\prime \prime}$ & $110^{\circ} 49^{\prime} 19.1^{\prime \prime}$ & 11 & 11 & 13 \\
\hline Unnamed hot spring drainage channel & 06WA116B & $44^{\circ} 31^{\prime} 2.1^{\prime \prime}$ & $110^{\circ} 49^{\prime} 20.3^{\prime \prime}$ & 11 & 11 & 14 \\
\hline Unnamed hot spring drainage channel & 06WA116C & $44^{\circ} 31^{\prime} 2.1^{\prime \prime}$ & $110^{\circ} 49^{\prime} 21.3^{\prime \prime}$ & 11 & 11 & 15 \\
\hline Unnamed hot spring drainage channel & 06WA116D & $44^{\circ} 31^{\prime} 1.8^{\prime \prime}$ & $110^{\circ} 49^{\prime} 22.9^{\prime \prime}$ & 11 & 11 & 16 \\
\hline \multicolumn{7}{|l|}{ Mud Volcano area } \\
\hline Sulphur Caldron & 06WA164 & $44^{\circ} 37^{\prime} 41.5^{\prime \prime}$ & $110^{\circ} 25^{\prime} 55.9^{\prime \prime}$ & 12 & 12 & 41 \\
\hline Sulphur Caldron & 07WA110 & $44^{\circ} 37^{\prime} 41.5^{\prime \prime}$ & $110^{\circ} 25^{\prime} 55.9^{\prime \prime}$ & 12 & 12 & 58 \\
\hline Sulphur Caldron & 08WA114 & $44^{\circ} 37^{\prime} 41.5^{\prime \prime}$ & $110^{\circ} 25^{\prime} 55.9^{\prime \prime}$ & 12 & 12 & 77 \\
\hline Turbulent Pool & 06WA165 & $44^{\circ} 37^{\prime} 42.4^{\prime \prime}$ & $110^{\circ} 25^{\prime} 57.3^{\prime \prime}$ & 12 & 12 & 42 \\
\hline Turbulent Pool & 07WA109 & $44^{\circ} 37^{\prime} 42.4^{\prime \prime}$ & $110^{\circ} 25^{\prime} 57.3^{\prime \prime}$ & 12 & 12 & 57 \\
\hline \multicolumn{7}{|l|}{ Washburn Hot Springs } \\
\hline Inkpot Spring \#1 & 06WA166 & $44^{\circ} 45^{\prime} 52.8^{\prime \prime}$ & $110^{\circ} 25^{\prime} 48.9^{\prime \prime}$ & 13 & 13 & 43 \\
\hline Inkpot Spring \#3 & 06WA167 & $44^{\circ} 45^{\prime} 53.2^{\prime \prime}$ & $110^{\circ} 25^{\prime} 48.1^{\prime \prime}$ & 13 & 13 & 43 \\
\hline Unnamed thermal feature 160 feet elevation above inkpots & 06WA169 & $44^{\circ} 46^{\prime} 0.5^{\prime \prime}$ & $110^{\circ} 45^{\prime} 45.1^{\prime \prime}$ & 13 & 13 & 44 \\
\hline Upper large hot pool, Inkpot Spring area & 06WA168 & $44^{\circ} 45^{\prime} 53.9^{\prime \prime}$ & $110^{\circ} 25^{\prime} 46.2^{\prime \prime}$ & 13 & 13 & 45 \\
\hline
\end{tabular}




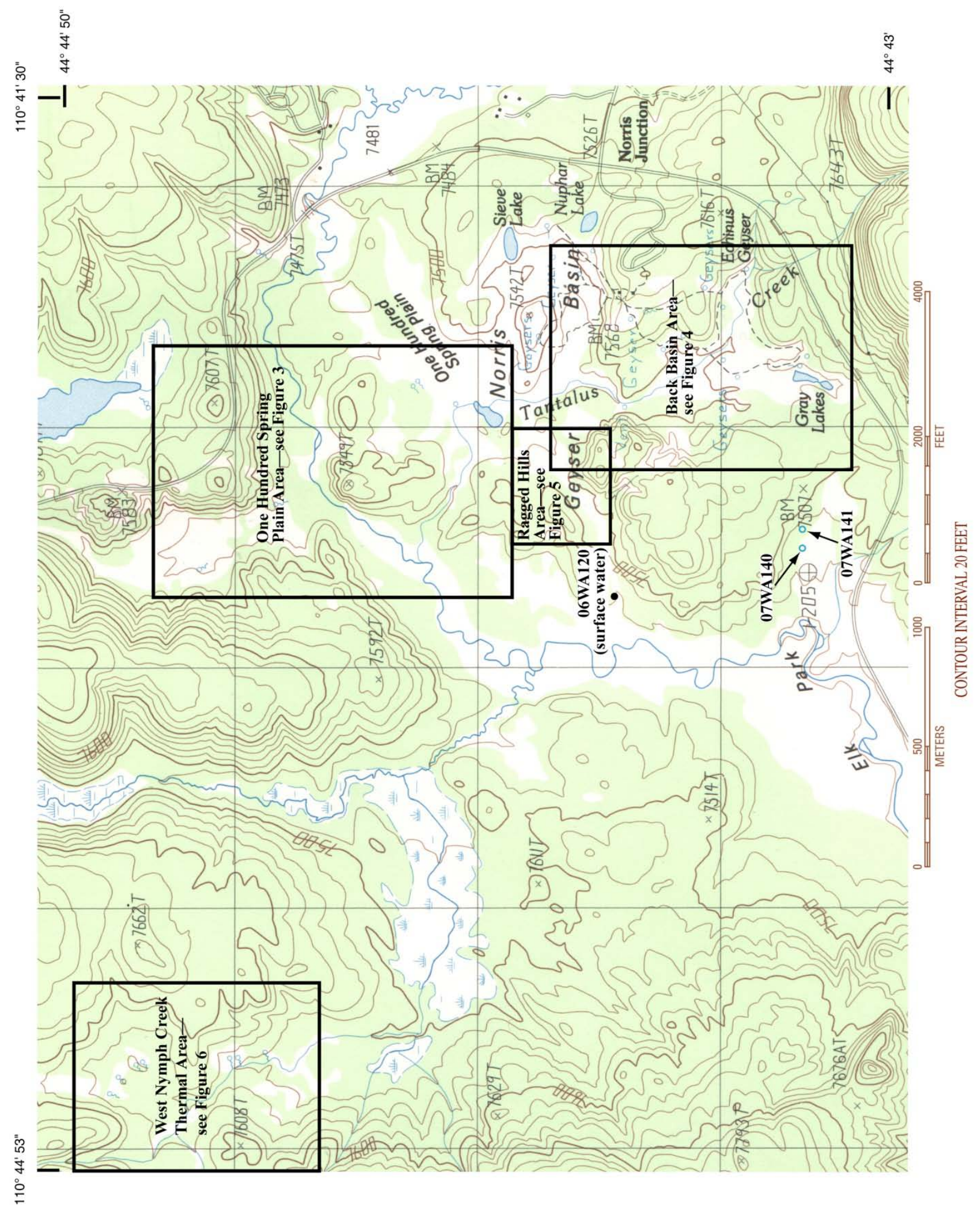

Base from U.S. Geological Survey Norris Junction quadrangle, 1:24,000 (1986)

Figure 2. Sampling locations for hot springs, geysers, and surface waters at Norris Geyser Basin and West Nymph Creek thermal area, Yellowstone National Park, Wyoming. 


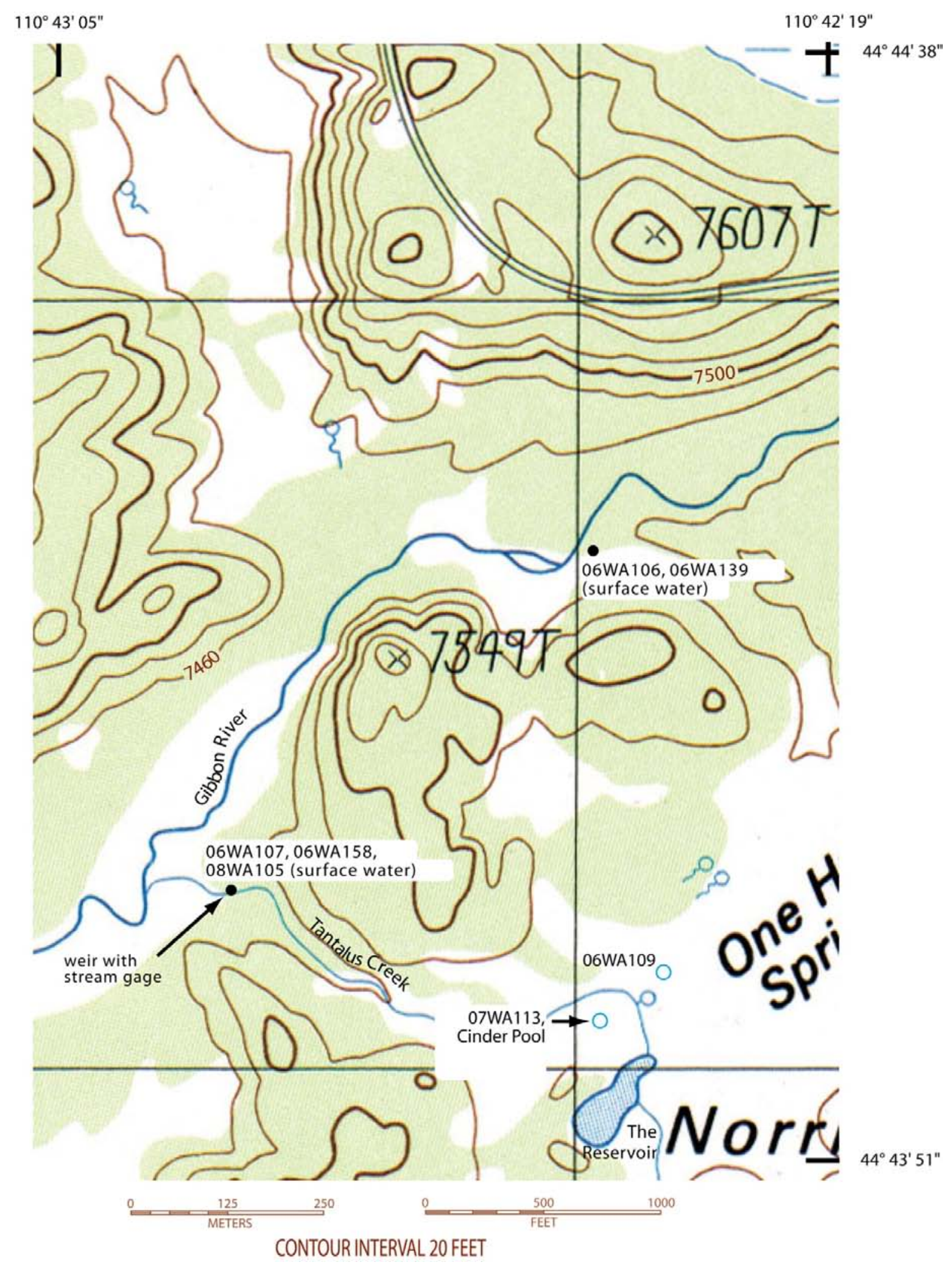

Base from U.S. Geological Survey Norris Junction quadrangle, 1:24,000 (1986)

Figure 3. Sampling locations with sample code numbers for hot springs and two surface-water samples in the One Hundred Spring Plain area of Norris Geyser Basin, Yellowstone National Park, Wyoming (see figure 2). 


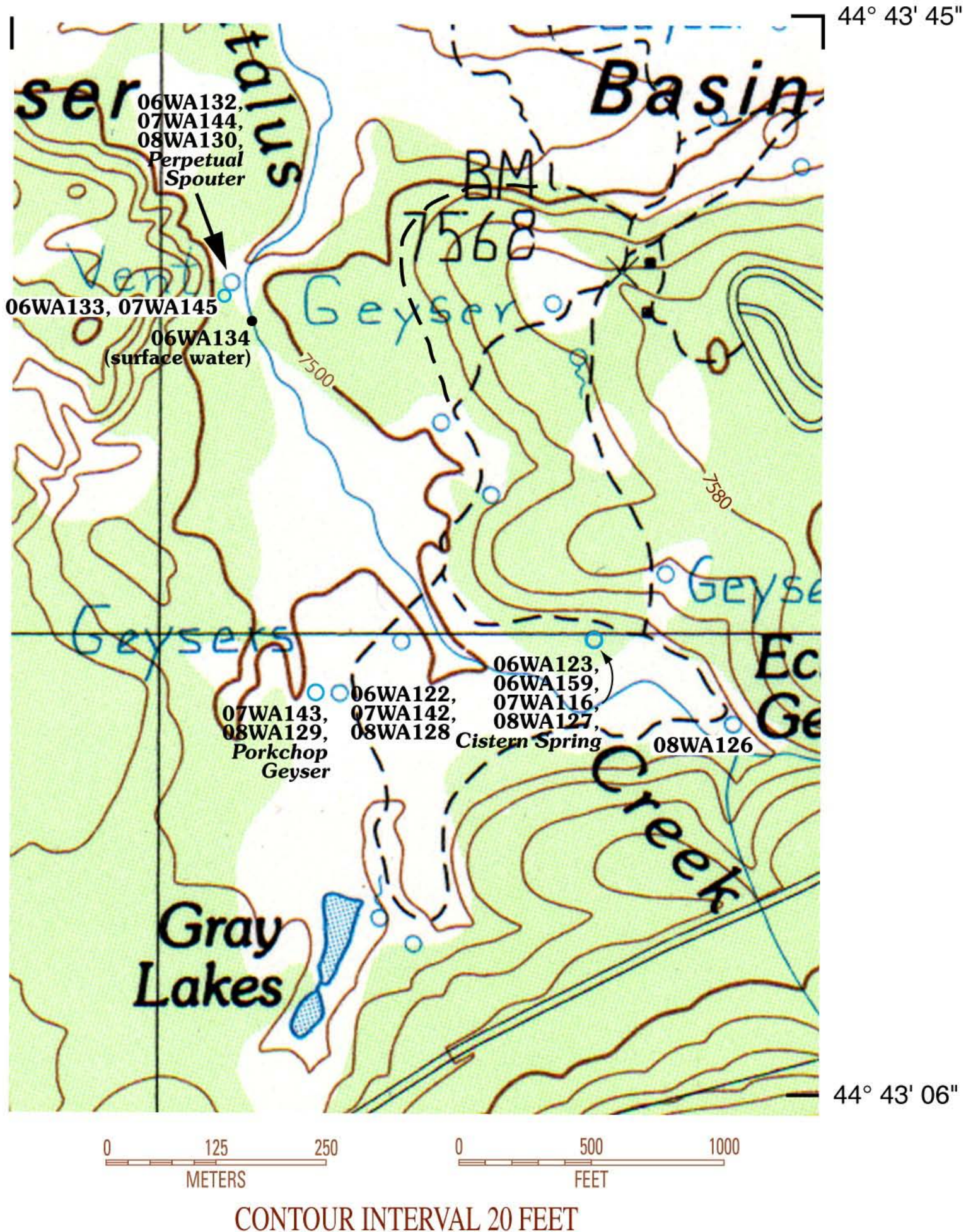

Base from U.S. Geological Survey Norris Junction quadrangle, 1:24,000 (1986)

Figure 4. Sampling locations with sample code numbers for thermal features and one surface-water sample in the Back Basin area of Norris Geyser Basin, Yellowstone National Park, Wyoming (see figure 2). 


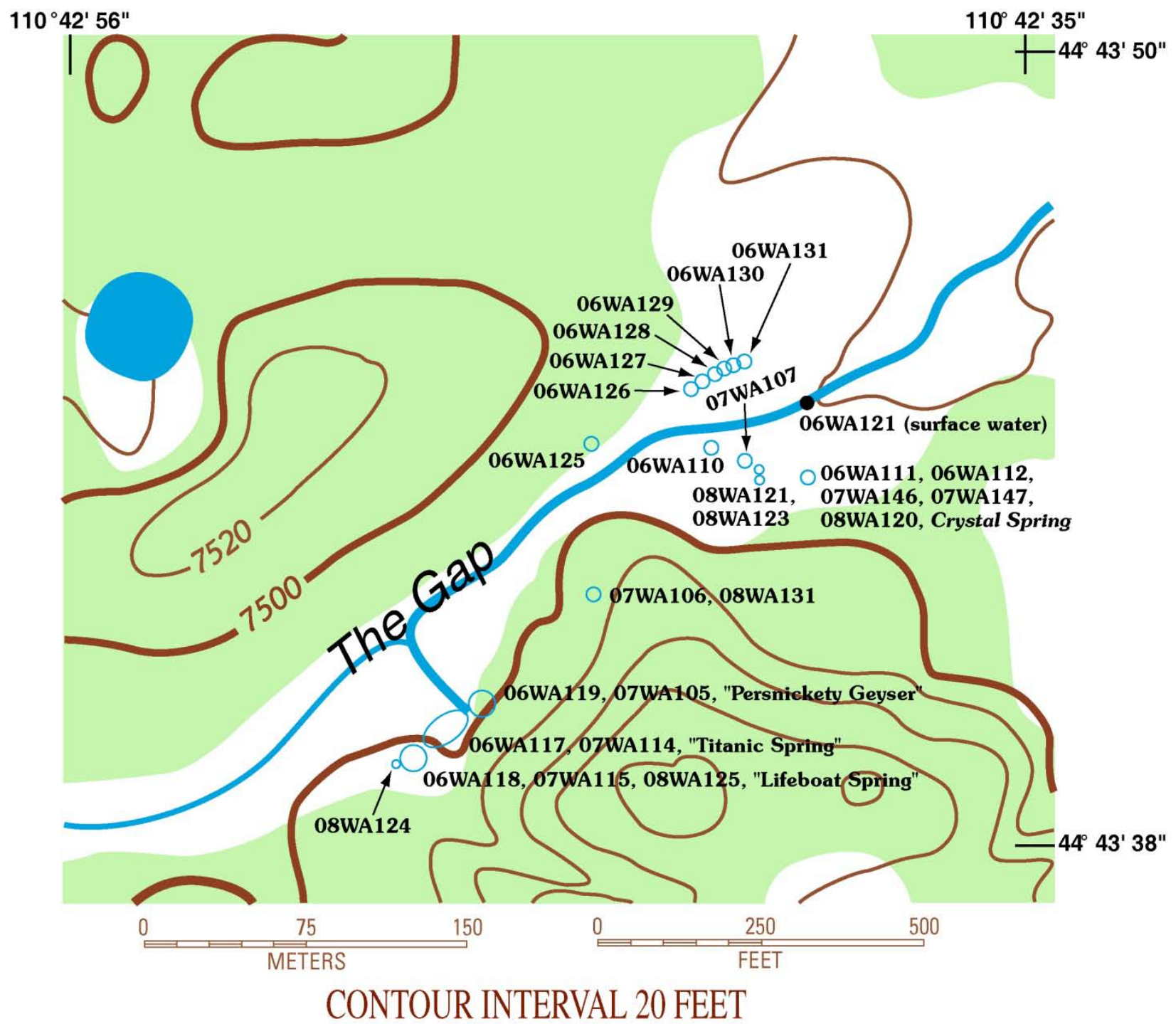

Sketch based on U.S. Geological Survey Norris Junction quadrangle, 1:24,000 (1986)

Figure 5. Sampling locations with sample code numbers for thermal features and one surface-water sample in the Ragged Hills area of Norris Geyser Basin, Yellowstone National Park, Wyoming (see figure 2). 


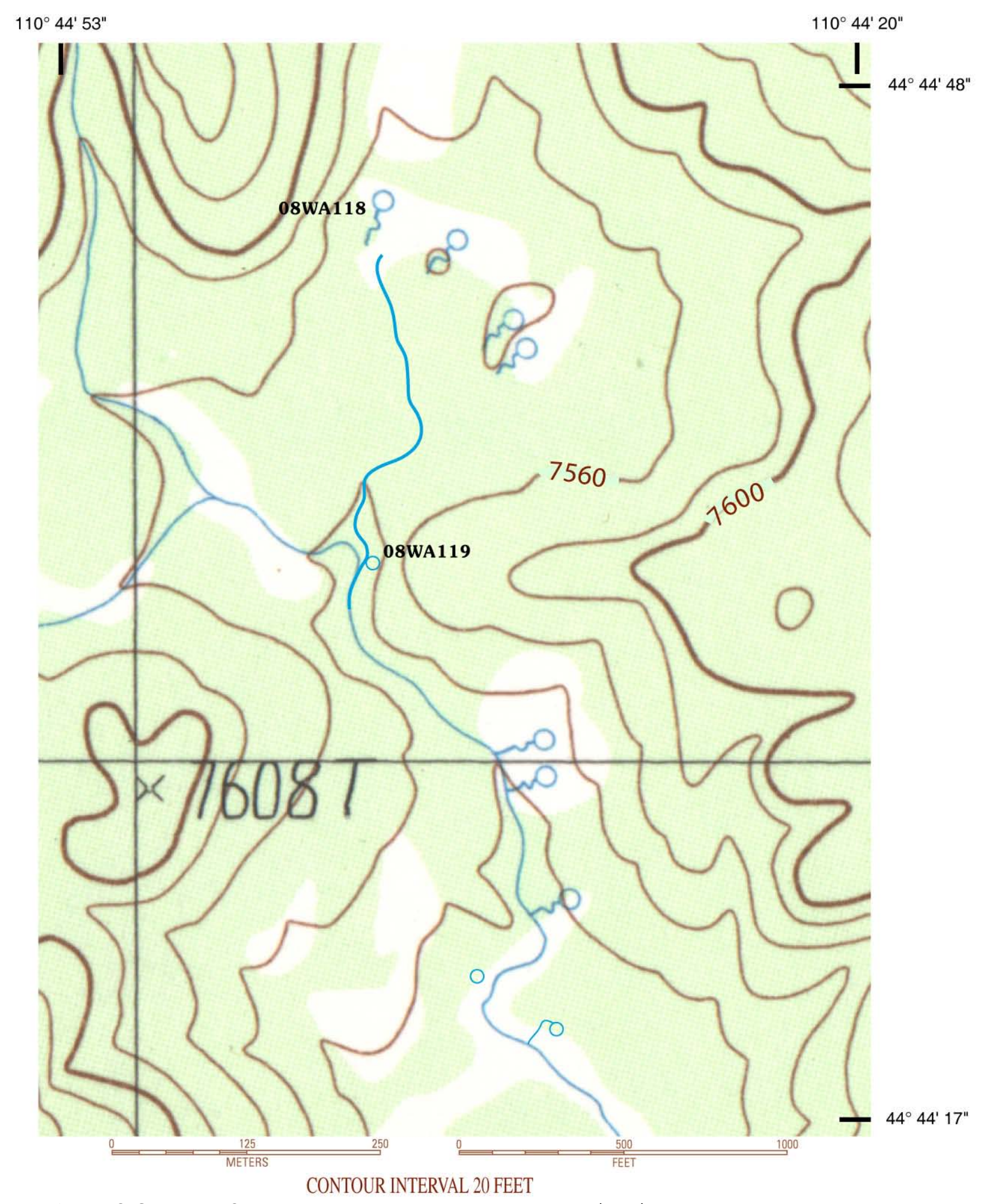

Base from U.S. Geological Survey Norris Junction quadrangle, 1:24,000 (1986)

Figure 6. Sampling locations with sample code numbers for thermal features in the West Nymph Creek Thermal Area near Norris Geyser Basin, Yellowstone National Park, Wyoming (see figure 2). 


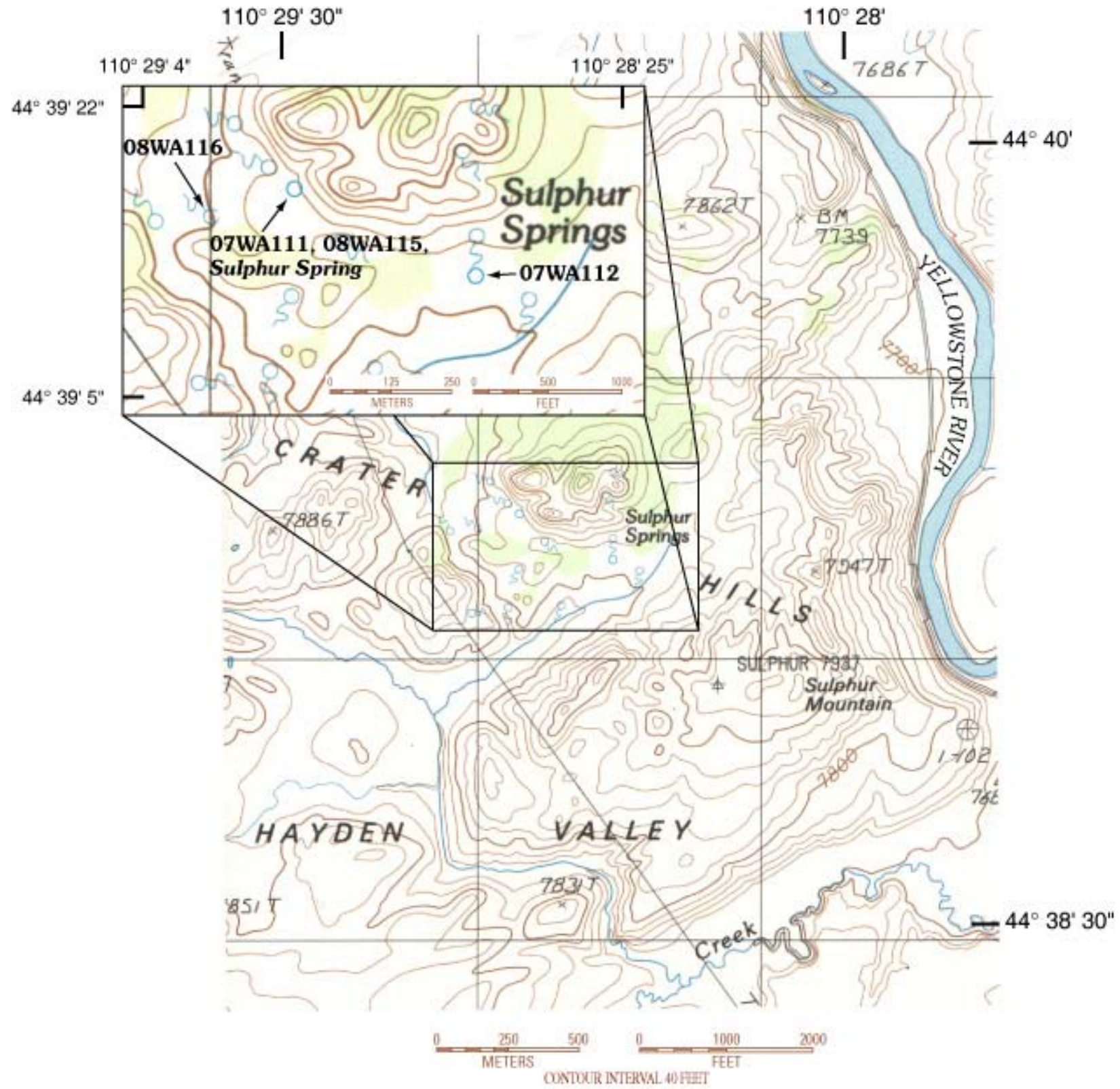

Base from U.S. Geological Survey Canyon Village quadrangle, 1:24,000 (1986)

Figure 7. Sampling locations with sample code numbers for thermal features at the Crater Hills area, Yellowstone National Park, Wyoming. 


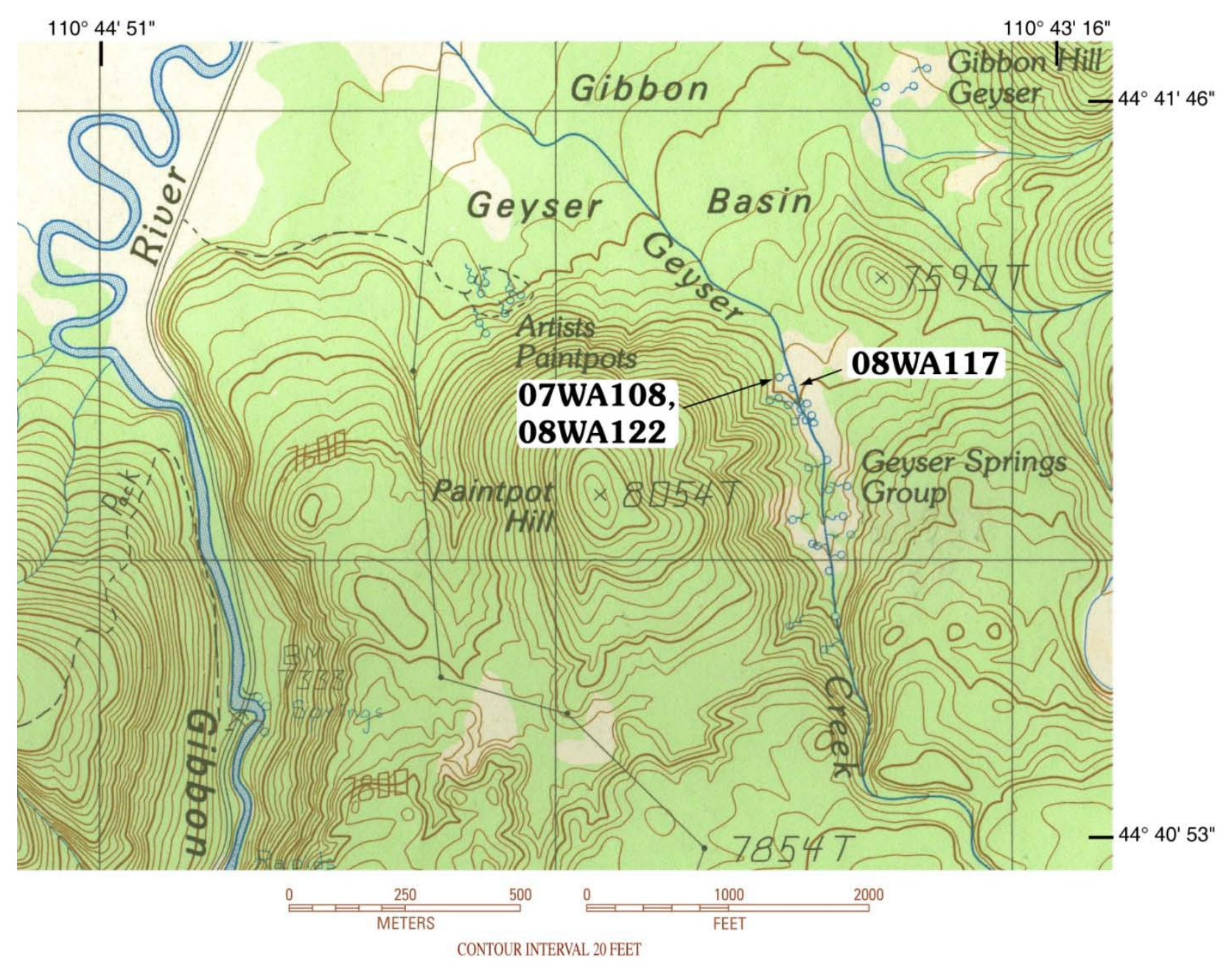

Base from U.S. Geological Survey Norris Junction quadrangle, 1:24,000 (1986)

Figure 8. Sampling location with sample code number for thermal features in the Geyser Springs Group, Yellowstone National Park, Wyoming. 


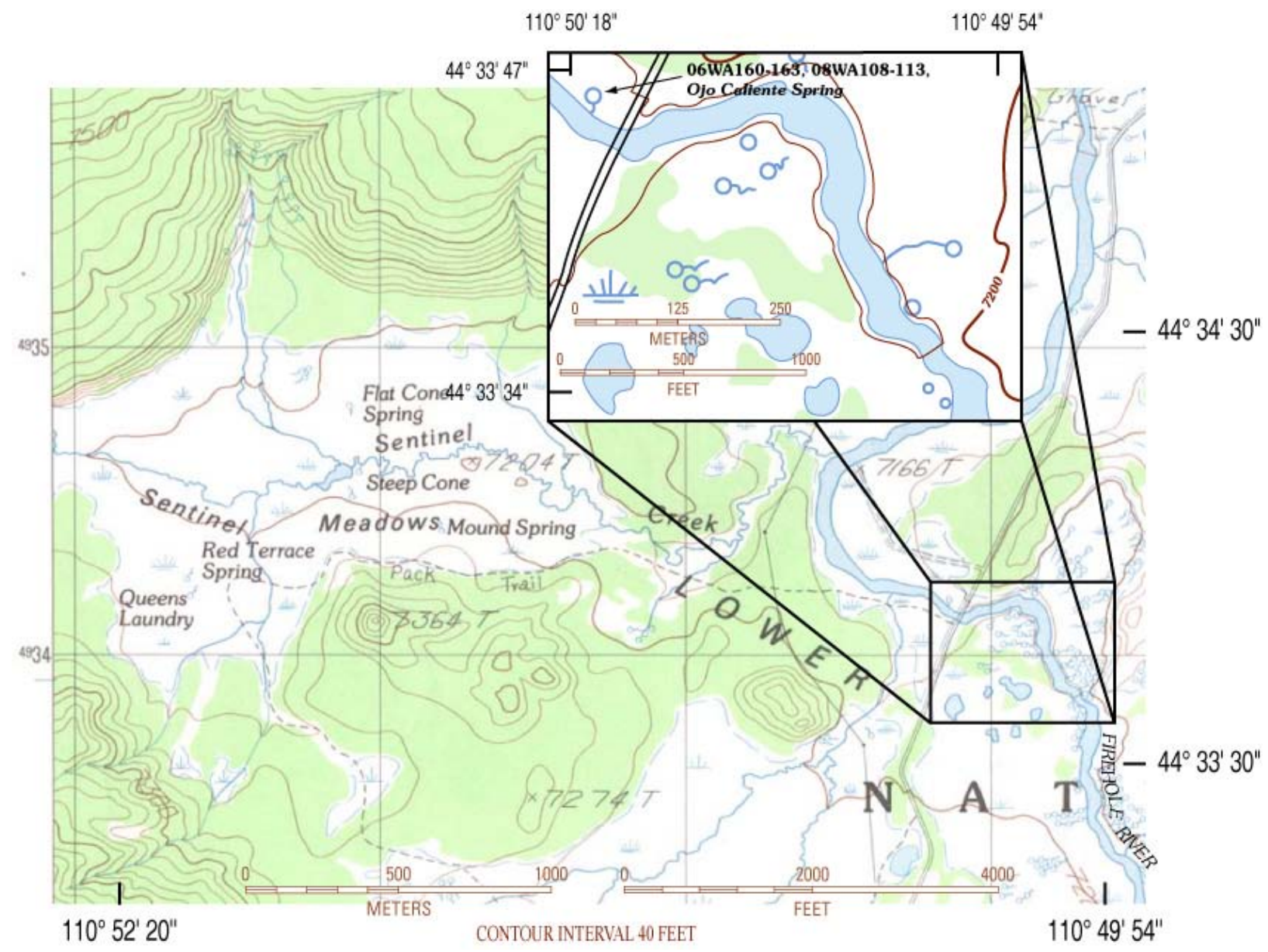

Base from U.S. Geological Survey Lower Geyser Basin quadrangle, 1:24,000 (1986)

Figure 9. Sampling location with sample code numbers for Ojo Caliente Spring in Lower Geyser Basin, Yellowstone National Park, Wyoming. 


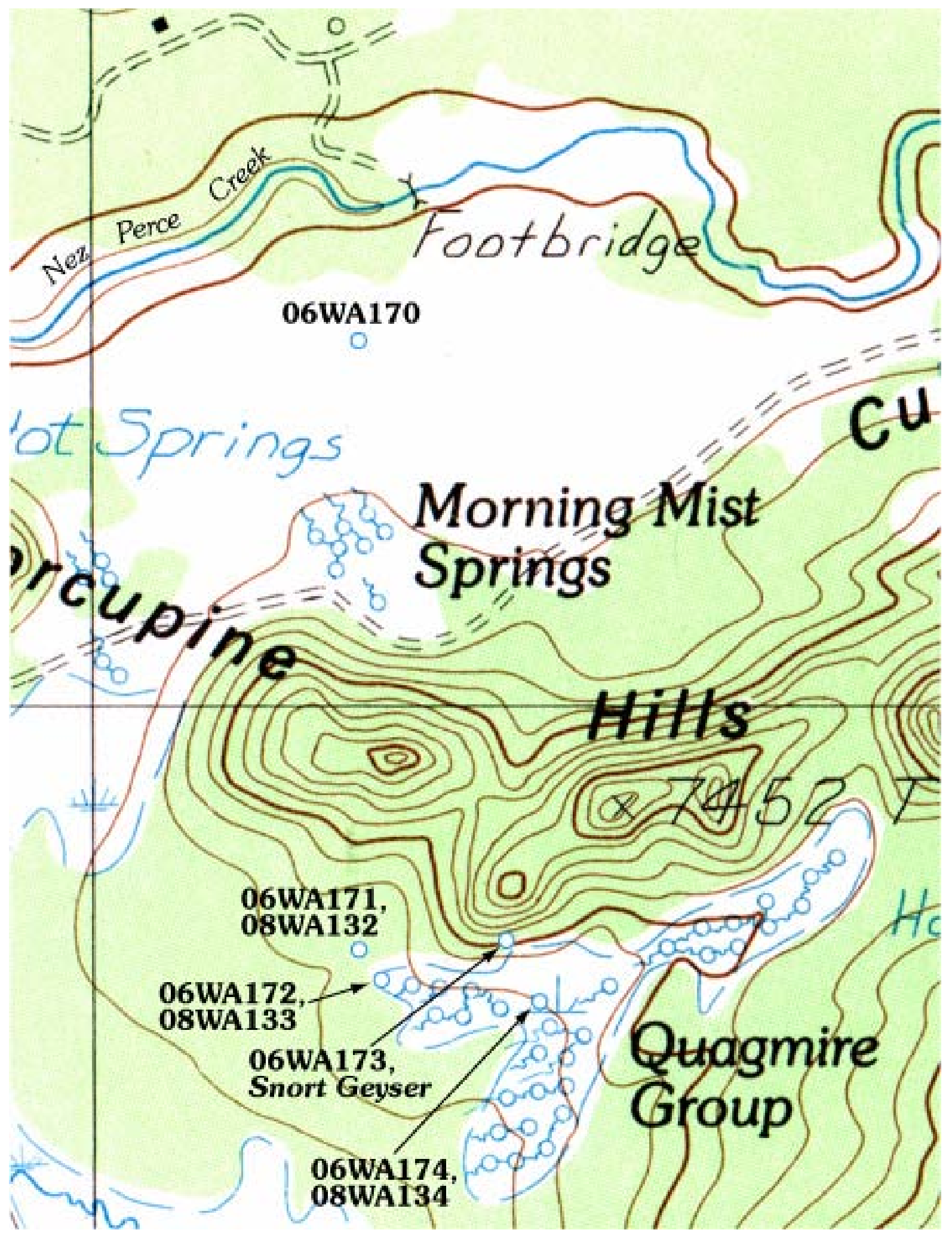

Base from U.S. Geological Survey Lower Geyser Basin quadrangle, 1:24,000 (1986)

Figure 10. Sampling locations with sample code numbers for thermal features in the Porcupine Hills area, Yellowstone National Park, Wyoming. 


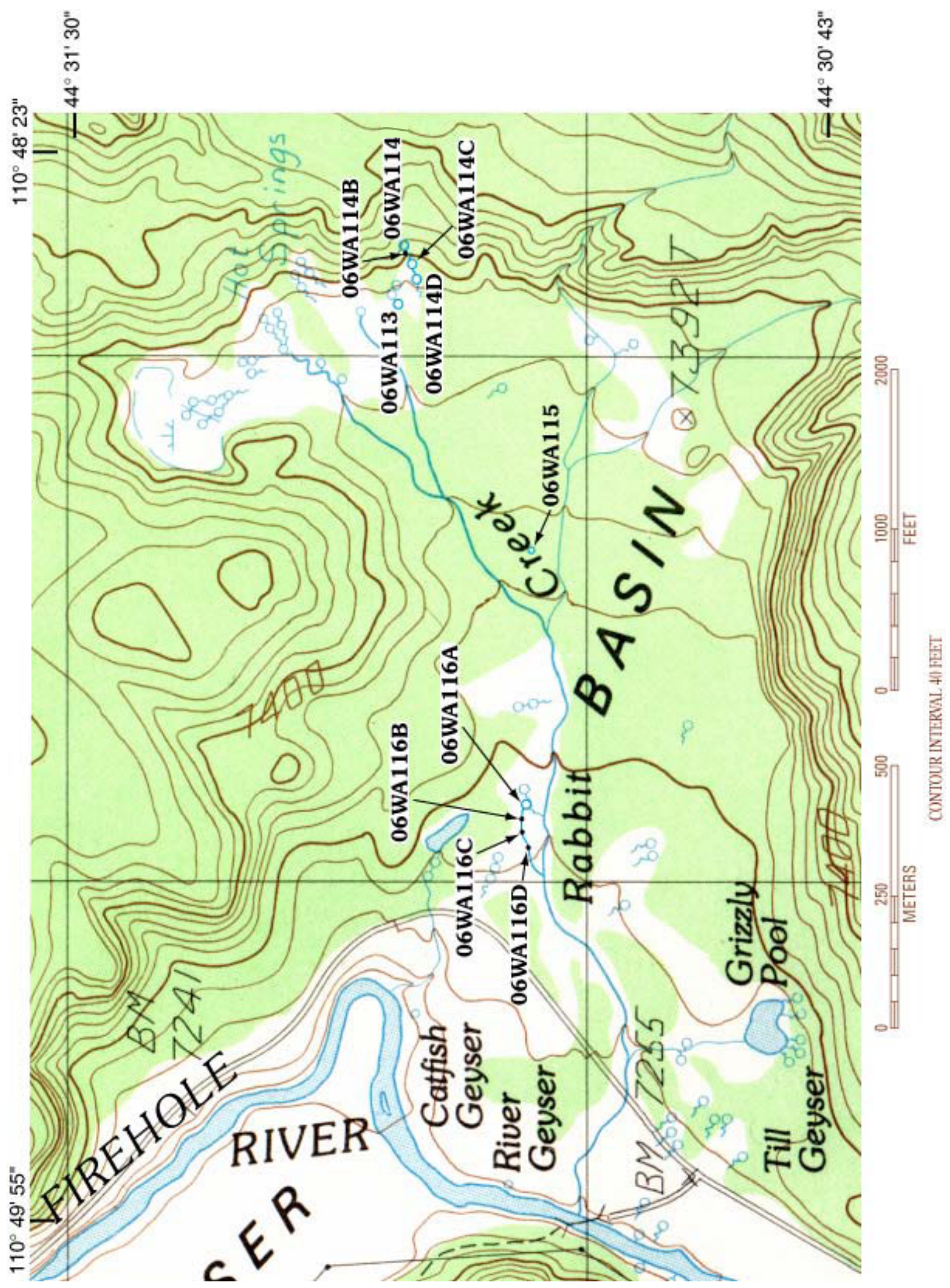

Base from U.S. Geological Survey Lower Geyser Basin quadrangle, 1:24,000 (1986)

Figure 11. Sampling locations with sample code numbers for the Rabbit Creek area, Yellowstone National Park, Wyoming. 


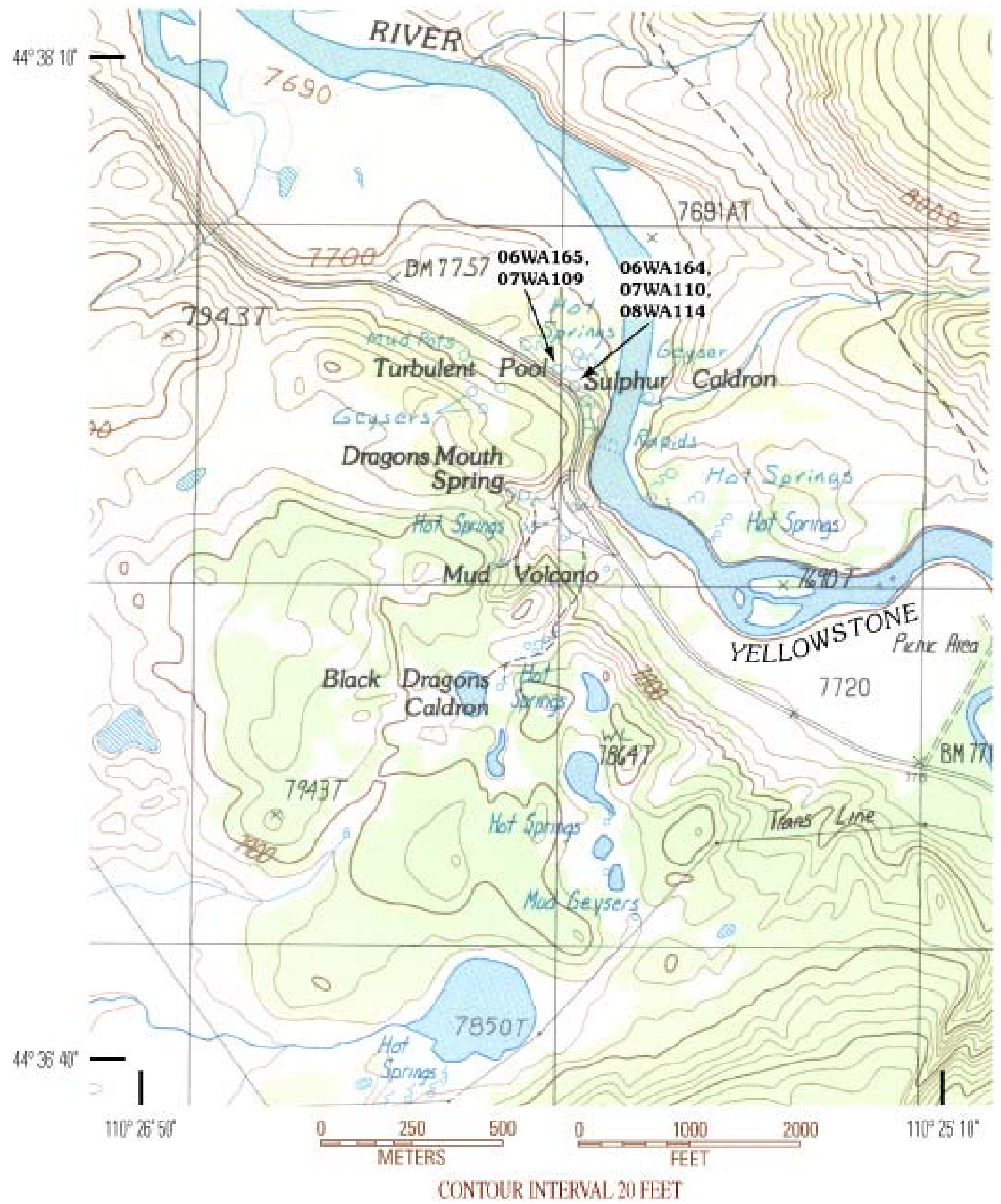

Base from U.S. Geological Survey Lake and Canyon Village quadrangles, 1:24,000 (1986)

Figure 12. Sampling locations with sample code numbers for thermal features in the Mud Volcano area, Yellowstone National Park, Wyoming. 


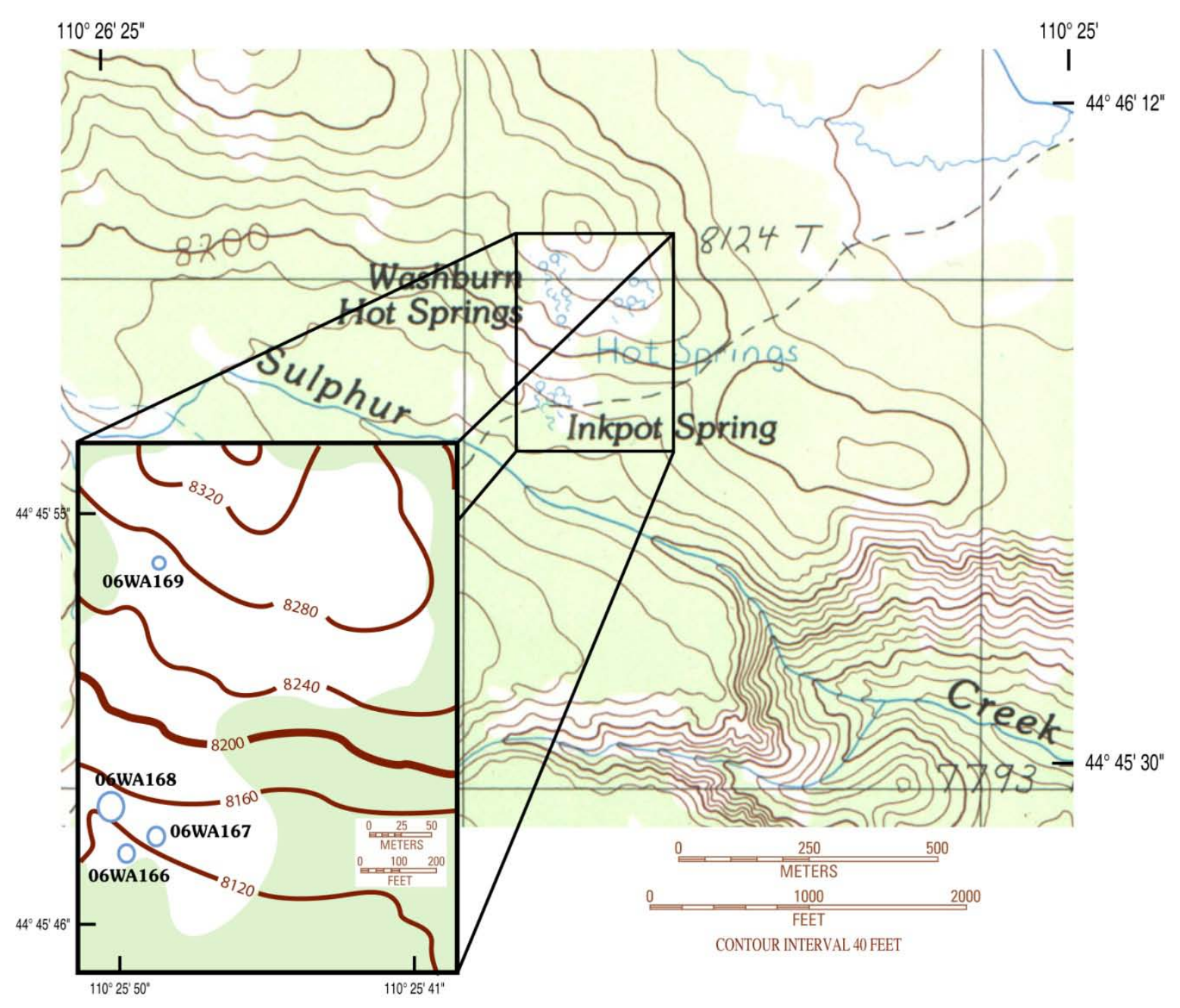

Base from U.S. Geological Survey Mount Washburn quadrangle, 1:24,000 (1986)

Figure 13. Sampling locations with sample code numbers for thermal features at Washburn Hot Springs, Yellowstone National Park, Wyoming. 
As many as 11 sample bottles were filled at each site. Sequential aliquots were filtered into separate containers for the determination of inorganic constituents, redox species, stable hydrogen and oxygen isotopes of water, and dissolved organic carbon (DOC). Container preparation, storage, and stabilization methods for filtered samples are summarized in table 2. Samples for the determination of major cations and trace metals (Al, $\mathrm{As}, \mathrm{B}, \mathrm{Ba}, \mathrm{Be}, \mathrm{Ca}, \mathrm{Cd}, \mathrm{Co}, \mathrm{Cr}, \mathrm{Cu}, \mathrm{Fe}, \mathrm{Hg}, \mathrm{K}$, $\mathrm{Li}, \mathrm{Mg}, \mathrm{Mn}, \mathrm{Mo}, \mathrm{Na}, \mathrm{Ni}, \mathrm{Pb}, \mathrm{Sb}, \mathrm{Se}, \mathrm{SiO}_{2}, \mathrm{Sr}, \mathrm{V}$, and $\mathrm{Zn}$ ), major anions (Br, Cl, F, $\mathrm{NO}_{3}$, and $\mathrm{SO}_{4}$ ), alkalinity, acidity, density, $\mathrm{NH}_{4}, \mathrm{DOC}$, and water isotopes $\left(\delta \mathrm{D}\right.$ and $\left.\delta^{18} \mathrm{O}\right)$ were filtered and then stabilizing reagents, if needed, were added. Stabilizing reagents for intermediate sulfur species were put into the sample bottles before the samples were collected; therefore, these bottles were not prerinsed. The remaining sample bottles were pre-rinsed with filtered water prior to sample collection. With the exception of the cation, water isotope, and silica dilution samples, all sample aliquots were chilled as soon as practical after sample collection.

To prevent over-estimation of $\mathrm{S}_{2} \mathrm{O}_{3}$ and polythionate concentrations, $\mathrm{S}(-\mathrm{II})$ oxidation was minimized by precipitating S(-II) with zinc chloride. This technique caused zinc sulfide (ZnS) species to precipitate. The $\mathrm{ZnS}$ in the samples was further stabilized by adding $\mathrm{NaOH}$. Polythionate was converted to thiocyanate (SCN) by adding potassium cyanide $(\mathrm{KCN})$ to that sample split (Moses and others, 1984). For the analysis of dissolved $\mathrm{SiO}_{2}$ in thermal waters, $1 \mathrm{~mL}$ of sample was diluted on】site to $25 \mathrm{~mL}$ with deionized water to minimize precipitation of $\mathrm{SiO}_{2}$ as the sample cooled. Samples for the determination of DOC were filtered through a 142-mm diameter all-plastic plate filter containing a $0.1-\mu \mathrm{m}$ mixed-cellulose-ester filter membrane and collected in a glass bottle that had been baked at $600^{\circ} \mathrm{C}$. At least 1 liter (L) of sample water was passed through the all-plastic plate-filter assembly before a DOC sample was collected. Samples for $\delta \mathrm{D}$ and $\delta^{18} \mathrm{O}$ (water isotopes) determinations were filtered when the water filtered easily; otherwise water-isotope samples were not filtered.

\section{Field and Laboratory Measurements}

Analytical methods, typical precision, detection limits, equipment used, pertinent references, and comments are described briefly in table 3. Detection limits were equal to 3 times the standard deviation (s) of several dozen measurements of the constituent in a blank solution analyzed as a sample. Relative standard deviation for each analytical method is determined from the measurement of an analyte concentration at least 10 times the detection limit. Techniques, general conditions, and variants from standard procedures are discussed in the following sections.

All reagents were equal to the reagent-grade standards of the American Chemical Society or more pure. Double-distilled or de-ionized water and re-distilled or trace-metal-grade acids were used in all preparations. Samples were diluted as necessary to bring the analyte concentration within the optimal range of the method. Each sample was analyzed at least twice for each dilution and for all constituents. 


\section{Table 2. Container preparation and stabilization methods for filtered samples.}

$\left[{ }^{\circ} \mathrm{C}\right.$, degrees Celsius; $\mathrm{HCO}_{3}$, bicarbonate; $\mathrm{HCl}$, hydrochloric acid; $\mathrm{HNO}_{3}$, nitric acid; $1: 9 \mathrm{H}_{2} \mathrm{SO}_{4}$, one part sulfuric acid plus nine parts water; $\mathrm{KCN}$, potassium cyanide; $\mathrm{K}_{2} \mathrm{Cr}_{2} \mathrm{O}_{7}$, potassium dichromate; $\mathrm{M}$, molar; mL, milliliter; $\mathrm{N}$, normal; $\mathrm{NaOH}$, sodium hydroxide; (S-II), sulfide; v/v, volume per volume; w/v, weight per volume; $\mathrm{ZnCl}_{2}, \mathrm{zinc}_{\mathrm{chloride}} \%$, percent]

\begin{tabular}{|c|c|c|}
\hline Constituent(s) to be determined & Storage container and preparation & $\begin{array}{l}\text { Stabilization treatment in addition to } \\
\text { refrigeration }\end{array}$ \\
\hline $\begin{array}{l}\text { Major cations and trace metals (Al, } \\
\mathrm{B}, \mathrm{Ba}, \mathrm{Be}, \mathrm{Ca}, \mathrm{Cd}, \mathrm{Co}, \mathrm{Cr}, \mathrm{Cu}, \mathrm{K}, \\
\mathrm{Li}, \mathrm{Mg}, \mathrm{Mn}, \mathrm{Mo}, \mathrm{Na}, \mathrm{Ni}, \mathrm{Pb}, \mathrm{Sb}(\mathrm{T}) \text {, } \\
\left.\mathrm{Sb}(\mathrm{III}), \mathrm{Se}, \mathrm{SiO}_{2}, \mathrm{Sr}, \mathrm{V}, \text { and } \mathrm{Zn}\right)\end{array}$ & $\begin{array}{l}\text { Polyethylene bottles, soaked in 5\% } \\
\mathrm{HCl} \text { and rinsed } 3 \text { times with } \\
\text { deionized water }\end{array}$ & $\begin{array}{l}1 \%(\mathrm{v} / \mathrm{v}) \text { concentrated redistilled } \\
\mathrm{HNO}_{3} \text { added; samples were not } \\
\text { chilled }\end{array}$ \\
\hline Total mercury $(\mathrm{Hg}(\mathrm{T}))$ & $\begin{array}{l}\text { Borosilicate glass bottles, soaked } \\
\text { with } 5 \% \mathrm{HNO}_{3} \text { and rinsed } 3 \text { times } \\
\text { with deionized water }\end{array}$ & $\begin{array}{l}1 \%(\mathrm{v} / \mathrm{v}) \text { concentrated redistilled } \\
\mathrm{HNO}_{3} / 1 \%(\mathrm{w} / \mathrm{v}) \mathrm{K}_{2} \mathrm{Cr}_{2} \mathrm{O}_{7} \text { added }\end{array}$ \\
\hline Methylmercury $\left(\mathrm{CH}_{3} \mathrm{Hg}\right)$ & $\begin{array}{l}\text { Amber borosilicate glass bottles, } \\
\text { soaked with } 5 \% \mathrm{HNO}_{3} \text { and rinsed } 3 \\
\text { times with deionized water }\end{array}$ & $5 \mathrm{~mL} 6 \mathrm{M}$ redistilled $\mathrm{HCl}$ added \\
\hline $\begin{array}{l}\text { Iron, arsenic, and antimony redox } \\
\text { species (Fe(T), } \mathrm{Fe}(\mathrm{II}), \mathrm{As}(\mathrm{T}), \\
\mathrm{As}(\mathrm{III}), \mathrm{Sb}(\mathrm{T}), \text { and } \mathrm{Sb}(\mathrm{III}))\end{array}$ & $\begin{array}{l}\text { Opaque polyethylene bottles, } \\
\text { soaked in } 5 \% \mathrm{HCl} \text { and rinsed } 3 \\
\text { times with deionized water }\end{array}$ & $1 \%(\mathrm{v} / \mathrm{v})$ redistilled $6 \mathrm{M} \mathrm{HCl}$ added \\
\hline $\begin{array}{l}\text { Major anions }\left(\mathrm{Br}, \mathrm{Cl}, \mathrm{F} \text {, and } \mathrm{SO}_{4}\right) \text {, } \\
\text { alkalinity as } \mathrm{HCO}_{3} \text {, acidity, } \\
\text { density, and nitrate }\left(\mathrm{NO}_{3}\right)\end{array}$ & $\begin{array}{l}\text { Polyethylene bottles filled with } \\
\text { distilled water and allowed to stand } \\
\text { for } 24 \text { hours, then rinsed } 3 \text { times } \\
\text { with deionized water }\end{array}$ & None \\
\hline Ammonium $\left(\mathrm{NH}_{4}\right)$ & $\begin{array}{l}\text { Same as major cations and trace } \\
\text { metals }\end{array}$ & $1 \%(\mathrm{v} / \mathrm{v}) 1: 9 \mathrm{H}_{2} \mathrm{SO}_{4}$ added \\
\hline Silica $\left(\mathrm{SiO}_{2}\right)$ & $\begin{array}{l}\text { Same as major anions, alkalinity, } \\
\text { and density }\end{array}$ & $\begin{array}{l}1 \mathrm{~mL} \text { diluted to } 25 \mathrm{~mL} \text { with } \\
\text { distilled water on } \square \text { site; samples } \\
\text { were not chilled }\end{array}$ \\
\hline $\begin{array}{l}\text { Thiosulfate }\left(\mathrm{S}_{2} \mathrm{O}_{3}\right) \text { and polythionate } \\
\left(\mathrm{S}_{\mathrm{n}} \mathrm{O}_{6}\right)\end{array}$ & 30-mL polyethylene bottle & $\begin{array}{l}1.7 \%(\mathrm{v} / \mathrm{v}) 0.6 \mathrm{M} \mathrm{ZnCl}_{2} \text { plus } 1 \% \\
\text { (v/v) } 1 \mathrm{M} \mathrm{NaOH} \text { added; } 1.7 \%(\mathrm{v} / \mathrm{v}) \\
1 \mathrm{M} \mathrm{KCN} \text { also added to } \mathrm{S}_{\mathrm{n}} \mathrm{O}_{6} \text { bottle }\end{array}$ \\
\hline Dissolved organic carbon (DOC) & Amber glass bottle baked at $600^{\circ} \mathrm{C}$ & None \\
\hline Water Isotopes $\left(\delta \mathrm{D}\right.$ and $\left.\delta^{18} \mathrm{O}\right)$ & 60-mL glass bottle & $\begin{array}{l}\text { None (unfiltered sample collected } \\
\text { when filtration was not possible); } \\
\text { samples were not chilled }\end{array}$ \\
\hline Sulfide & 30-mL polyethylene bottle & $\begin{array}{l}\text { 15-mL sulfide anti-oxidant buffer } \\
\text { followed by } 15-\mathrm{mL} \text { sample }\end{array}$ \\
\hline
\end{tabular}




\section{Field Measurements}

Measurements of temperature, $\mathrm{pH}$, Eh, specific conductance, dissolved oxygen (DO), and $\mathrm{H}_{2} \mathrm{~S}$ were performed on\site . Measurements of Eh and $\mathrm{pH}$ were made on unfiltered sample water pumped from the source through an acrylic plastic flow-through cell, which minimized sample contact with air. The flow-through cell contained a combination redox electrode, a combination $\mathrm{pH}$ electrode, a thermistor, and test tubes containing buffer solutions for calibration of the $\mathrm{pH}$ electrode. All components were thermally equilibrated with the sample water before obtaining measurements. Where possible, specific conductance and sampling tube intake temperature were measured by immersing the combined specific conductance/temperature probe directly into the source as close to the sampling point as possible. Otherwise, the probe was immersed in the flow-through cell. Because sample temperatures usually were greater than the upper limit $\left(45^{\circ} \mathrm{C}\right)$ of the DO probe, DO was determined using the azide modification of the Winkler titration (American Public Health Association (APHA), 1971).

A mobile laboratory truck containing an ion chromatograph, ultraviolet-visible spectrophotometer, autotitrator, and reagent-grade water system was set up at the overnight lodging site so that unstable intermediate sulfur oxyanion species could be determined as soon as possible after sample collection. Iron redox species also were determined in the mobile laboratory from time to time to guide sampling strategies. The autotitrator was used each evening to perform alkalinity and acidity titrations before oxidation and hydrolysis reactions occurred, and to determine $\mathrm{H}_{2} \mathrm{~S}$ concentrations in samples preserved on $\square$ site.

\section{$\mathrm{pH}$ Measurements}

Because field measurement of $\mathrm{pH}$ in geothermal waters is challenging and accurate $\mathrm{pH}$ measurements are critical for interpreting analytical results (Ball and others, 2006), special care was taken when measuring this parameter. At each site, the flow-through cell, temperature probe, electrode, and calibration buffers were equilibrated to sample temperature prior to calibration and measurement. The system was calibrated using at least two bracketing standard buffers (chosen from among 1.68, 4.01, 7.00, or 10.00) corrected to their values at the sample temperature. After calibration, the $\mathrm{pH}$ electrode was placed in the sample water in the flow-through cell and monitored until no change in temperature $\left( \pm 0.1^{\circ} \mathrm{C}\right)$ or $\mathrm{pH}( \pm 0.01$ standard unit) was detected for at least 30 seconds. Following sample measurement, the electrode was immersed in the standard buffer of $\mathrm{pH}$ closest to that of the sample and allowed to equilibrate. The entire calibration and measurement process was repeated as many times as necessary until the measured value for the buffer differed by no more than 0.05 standard units from its certified $\mathrm{pH}$ at the measured temperature. 
Table 3. Analytical techniques, detection limits, typical precision, equipment used, and analytical method references.

[cm, centimeter; Cs, cesium; CVAFS, cold vapor atomic fluorescence spectrometry; ${ }^{\circ} \mathrm{C}$, degrees Celsius; GFAAS, graphite furnace atomic absorption spectrometry; $\mathrm{HCl}$, hydrochloric acid; HGAAS, hydride generation atomic absorption spectrometry; IC, ion chromatography; ICP-OES, inductively coupled plasma-optical emission spectrometry; ISE, ion-selective electrode; $\mathrm{KCl}$, potassium chloride; $\mathrm{KI}$, potassium iodide; $\mathrm{M}$, molar; $\mathrm{Mg}\left(\mathrm{NO}_{3}\right)_{2}$, magnesium nitrate; $\mu \mathrm{g} / \mathrm{L}$, micrograms per liter; $\mathrm{mg} / \mathrm{L}$, milligrams per liter; mM, millimolar; mN, millinormal; MS, mass spectrometry; $\mathrm{N}$, normal; $\mathrm{NaHCO}_{3}$, sodium bicarbonate; $\mathrm{Na}_{2} \mathrm{CO}_{3}$, sodium carbonate; ng $/ \mathrm{L}$, nanograms per liter; nm, nanometer; Pd, palladium; RSD, relative standard deviation; SLAP, standard light antarctic precipitation; TISAB, total ionic strength adjustment buffer; TOC, total organic carbon; VSMOW, Vienna standard mean ocean water; $\leq$, less than or equal to; \%, percent]

\begin{tabular}{|c|c|c|c|c|}
\hline Constituent & Analytical Technique & $\begin{array}{l}\text { Typical RSD }{ }^{1} \\
\text { detection limit } \\
\text { (mg/L) }\end{array}$ & Equipment Used & Comments or Reference(s) \\
\hline \multicolumn{5}{|c|}{ Field Measurements } \\
\hline Temperature & Electronic sensor & Not applicable & $\begin{array}{l}\text { Beckman } 265 \mathrm{pH} \text { meter with Beckman } \\
\text { temperature probe or Orion Research model } \\
1230 \text { multi-parameter meter with temperature } \\
\text { sensor built into conductivity electrode }\end{array}$ & Temperatures were reported to the nearest $0.1^{\circ} \mathrm{C}$ \\
\hline $\mathrm{pH}$ & Potentiometry & $0.02 \mathrm{pH}$ units $^{2}$ & $\begin{array}{l}\text { Beckman } 265 \mathrm{pH} \text { meter with Orion Ross } \\
\text { combination electrode }\end{array}$ & $\begin{array}{l}\text { Two- or three-buffer calibration at sample } \\
\text { temperature using } 1.68,4.01,7.00 \text {, and } 10.00 \mathrm{pH} \\
\text { buffers }\end{array}$ \\
\hline $\begin{array}{l}\text { Specific } \\
\text { Conductance } \\
\text { (SC) }\end{array}$ & Conductometry & $0.5 \%$ & $\begin{array}{l}\text { Orion Research model } 1230 \text { multi-parameter } \\
\text { meter with conductivity electrode }\end{array}$ & $\begin{array}{l}\text { Automatic temperature correction to } 25^{\circ} \mathrm{C} \text {, cell } \\
\text { constant determined with } 0.0100 \mathrm{~N} \mathrm{KCl}\end{array}$ \\
\hline Eh & Potentiometry & $10 \%$ & $\begin{array}{l}\text { Orion Research model 96-78-00 combination } \\
\text { redox electrode }\end{array}$ & $\begin{array}{l}\text { Electrode checked using ZoBell's solution } \\
\text { (ZoBell, 1946; Nordstrom, 1977) }\end{array}$ \\
\hline $\begin{array}{l}\text { Dissolved } \\
\text { oxygen } \\
\text { (DO) }\end{array}$ & Titration & $1 \%$ & Burette and Erlenmeyer flask & $\begin{array}{l}\text { Winkler Titration using manganous sulfate, } \\
\text { alkaline iodide-azide, sulfamic acid, starch } \\
\text { indicator, phenyl arsine oxide (APHA, 1971) }\end{array}$ \\
\hline $\begin{array}{l}\text { Hydrogen } \\
\text { sulfide } \\
\left(\mathrm{H}_{2} \mathrm{~S}\right)\end{array}$ & Colorimetry / & $\leq 20 \%, 0.002$ & $\begin{array}{l}\text { Hach model DR-2000 or DR- } 2800 \\
\text { ultraviolet - visible spectrometer and Hach } \\
\text { method \# } 8131 \text { reagents / }\end{array}$ & Methylene Blue Method based on APHA (1985) \\
\hline \multicolumn{5}{|c|}{ Laboratory Measurements } \\
\hline Calcium $(\mathrm{Ca})$ & ICP-OES & $2 \%, 0.04$ & Leeman Labs Direct Reading Echelle ${ }^{3}$ & Wavelength: $315.887 \mathrm{~nm}$, view: radial \\
\hline $\begin{array}{l}\text { Magnesium } \\
(\mathrm{Mg})\end{array}$ & ICP-OES & $2 \%, 0.001$ & Leeman Labs Direct Reading Echelle ${ }^{3}$ & Wavelength: $280.270 \mathrm{~nm}$, view: axial \\
\hline Sodium $(\mathrm{Na})$ & ICP-OES & $2 \%, 0.1$ & Leeman Labs Direct Reading Echelle ${ }^{3}$ & Wavelength: $589.592 \mathrm{~nm}$, view: radial \\
\hline
\end{tabular}


Table 3. Analytical techniques, detection limits, typical precision, equipment used, and analytical method references—Continued.

\begin{tabular}{|c|c|c|c|c|}
\hline Constituent & Analytical Technique & $\begin{array}{l}\text { Typical RSD1, } \\
\text { detection limit } \\
\quad(\mathrm{mg} / \mathrm{L})\end{array}$ & Equipment Used & Comments or Reference(s) \\
\hline $\begin{array}{l}\text { Potassium } \\
(\mathrm{K})\end{array}$ & ICP-OES & $3 \%, 0.04$ & Leeman Labs Direct Reading Echelle ${ }^{3}$ & $\begin{array}{l}\text { 1,000 mg/L Cs ionization buffer, } \\
\text { Wavelength: } 766.490 \mathrm{~nm} \text {, view: axial }\end{array}$ \\
\hline $\begin{array}{l}\text { Strontium } \\
(\mathrm{Sr})\end{array}$ & ICP-OES & $2 \%, 0.0007$ & Leeman Labs Direct Reading Echelle ${ }^{3}$ & Wavelength: $421.611 \mathrm{~nm}$, view: axial \\
\hline Barium $(\mathrm{Ba})$ & ICP-OES & $2 \%, 0.0008$ & Leeman Labs Direct Reading Echelle ${ }^{3}$ & Wavelength: $455.403 \mathrm{~nm}$, view: axial \\
\hline Lithium (Li) & ICP-OES & $2 \%, 0.003$ & Leeman Labs Direct Reading Echelle ${ }^{3}$ & $\begin{array}{l}\text { 1,000 mg/L Cs ionization buffer, } \\
\text { Wavelength: } 670.784 \mathrm{~nm} \text {, view: axial }\end{array}$ \\
\hline Sulfate $\left(\mathrm{SO}_{4}\right)$ & $\mathrm{IC}$ & $3 \%, 1.0$ & $\begin{array}{l}\text { Dionex model } 100 \text { or } 600 \text { ion chromatograph with } \\
\text { AG22A guard and AS22A separator columns and } \\
\text { Anion Self-Regenerating Suppressor-II }\end{array}$ & $\begin{array}{l}3.5 \mathrm{mM} \mathrm{NaHCO} \\
\text { eluent } \\
\text { (Brinton and others, 1995) }\end{array}$ \\
\hline $\begin{array}{l}\text { Thiosulfate } \\
\left(\mathrm{S}_{2} \mathrm{O}_{3}\right)\end{array}$ & $\mathrm{IC}$ & $3 \%, 0.1$ & $\begin{array}{l}\text { Dionex model } 100 \text { ion chromatograph with two AG4A } \\
\text { guard and an Anion Self-Regenerating Suppressor-II }\end{array}$ & $\begin{array}{l}0.028 \mathrm{M} \mathrm{NaHCO}_{3}+0.022 \mathrm{M} \mathrm{Na}_{2} \mathrm{CO}_{3} \\
\text { eluent (Moses and others, 1984) }\end{array}$ \\
\hline $\begin{array}{l}\text { Polythionate } \\
\left(\mathrm{S}_{\mathrm{n}} \mathrm{O}_{6}\right)\end{array}$ & $\mathrm{IC}$ & $3 \%, 0.3$ & $\begin{array}{l}\text { Dionex model } 100 \text { ion chromatograph with two AG4A } \\
\text { guard and an Anion Self-Regenerating Suppressor-II }\end{array}$ & $\begin{array}{l}0.028 \mathrm{M} \mathrm{NaHCO}_{3}+0.022 \mathrm{M} \mathrm{Na}_{2} \mathrm{CO}_{3} \\
\text { eluent (Moses and others, 1984) }\end{array}$ \\
\hline $\begin{array}{l}\text { Alkalinity } \\
\left(\text { as } \mathrm{HCO}_{3} \text { ) }\right.\end{array}$ & Titration & $2 \%, 1.0$ & $\begin{array}{l}\text { Orion Research model 960/940 autotitrator, } \\
\text { potentiometric detection, end-point determined by the } \\
\text { first derivative technique }\end{array}$ & $\begin{array}{l}\text { (Barringer and Johnsson, 1996; Fishman } \\
\text { and Friedman, 1989) }\end{array}$ \\
\hline $\begin{array}{l}\text { Acidity } \\
\text { (total/free } \mathrm{H}^{+} \text {) }\end{array}$ & Titration & $2 \%, 0.4 \mathrm{mM}$ & $\begin{array}{l}\text { Orion Research model 960/940 autotitrator, } \\
\text { potentiometric detection }\end{array}$ & $\begin{array}{l}\text { (Barringer and Johnsson, 1996; Fishman } \\
\text { and Friedman, 1989) }\end{array}$ \\
\hline $\begin{array}{l}\text { Hydrogen } \\
\text { sulfide }\left(\mathrm{H}_{2} \mathrm{~S}\right)\end{array}$ & ISE & $5 \%, 0.002$ & $\begin{array}{l}\text { Orion Research model 960/940 autotitrator, Accumet } \\
\text { model 13-620-551 Silver/Sulfide combination } \\
\text { electrode }\end{array}$ & $\begin{array}{l}\text { Sample mixed 1:1 on } \square \text { site with Orion } \\
\text { Research 941609 Sulfide Anti-Oxidant } \\
\text { Buffer and analyzed within } 24 \text { hours of } \\
\text { collection (APHA, 1998) }\end{array}$ \\
\hline Fluoride (F) & $\begin{array}{l}\text { ISE; } \\
\text { IC }\end{array}$ & $\begin{array}{l}3 \%, 0.05 \\
3 \%, 0.15\end{array}$ & $\begin{array}{l}\text { Orion Research model } 96-09 \text { combination F electrode; } \\
\text { Dionex model } 100 \text { or } 600 \text { ion chromatograph with } \\
\text { AG22A guard and AS22A separator columns and } \\
\text { Anion Self-Regenerating Suppressor-II }\end{array}$ & $\begin{array}{l}\text { Sample mixed 1:1 with Orion Research } \\
940911 \text { TISAB III (Barnard and } \\
\text { Nordstrom, 1980); } \\
3.5 \mathrm{mM} \mathrm{NaHCO}+1.0 \mathrm{mM} \mathrm{Na}_{2} \mathrm{CO}_{3} \\
\text { eluent (Brinton and others, 1995) }\end{array}$ \\
\hline Chloride $(\mathrm{Cl})$ & $\mathrm{IC}$ & $3 \%, 0.05$ & $\begin{array}{l}\text { Dionex model } 100 \text { or } 600 \text { ion chromatograph with } \\
\text { AG14A guard and AS14A separator columns and an } \\
\text { Anion Self-Regenerating Suppressor-II }\end{array}$ & $\begin{array}{l}3.5 \mathrm{mM} \mathrm{NaHCO} \\
\text { eluent } \\
\text { (Brinton and others, } 1995 \text { ) }\end{array}$ \\
\hline
\end{tabular}


Table 3. Analytical techniques, detection limits, typical precision, equipment used, and analytical method references-Continued.

\begin{tabular}{|c|c|c|c|c|}
\hline Constituent & Analytical Technique & $\begin{array}{l}\text { Typical RSD1, } \\
\text { detection limit } \\
\quad \text { (mg/L) }\end{array}$ & Equipment Used & Comments or Reference(s) \\
\hline Bromide (Br) & IC & $3 \%, 0.03$ & $\begin{array}{l}\text { Dionex model } 100 \text { or } 600 \text { ion chromatograph with } \\
\text { AG14A guard and AS14A separator columns and } \\
\text { an Anion Self-Regenerating Suppressor-II }\end{array}$ & $\begin{array}{l}3.5 \mathrm{mM} \mathrm{NaHCO}_{3}+1.0 \mathrm{mM} \mathrm{Na}_{2} \mathrm{CO}_{3} \text { eluent } \\
\text { (Brinton and others, } 1995 \text { ) }\end{array}$ \\
\hline Nitrate $\left(\mathrm{NO}_{3}\right)$ & $\mathrm{IC}$ & $3 \%, 0.1$ & $\begin{array}{l}\text { Dionex model } 100 \text { or } 600 \text { ion chromatograph with } \\
\text { AG22A guard and AS22A separator columns and } \\
\text { Anion Self-Regenerating Suppressor-II }\end{array}$ & $\begin{array}{l}3.5 \mathrm{mM} \mathrm{NaHCO}_{3}+1.0 \mathrm{mM} \mathrm{Na}_{2} \mathrm{CO}_{3} \text { eluent } \\
\text { (Brinton and others, } 1995 \text { ) }\end{array}$ \\
\hline $\begin{array}{l}\text { Ammonium } \\
\left(\mathrm{NH}_{4}\right)\end{array}$ & Colorimetry & $3 \%, 0.05$ & $\begin{array}{l}\text { Dionex model DX-300 ion chromatograph with } \\
\text { CS12A IonPac column }\end{array}$ & $\begin{array}{l}22 \mathrm{mN} \mathrm{H}_{2} \mathrm{SO}_{4} \text { eluent } \\
\text { (Smith and others, 2006) }\end{array}$ \\
\hline Silica $\left(\mathrm{SiO}_{2}\right)$ & ICP-OES & $2 \%, 0.05$ & Leeman Labs Direct Reading Echelle $^{3}$ & $\begin{array}{l}\text { Sample diluted 25X in field, Wavelength: } \\
251.611 \mathrm{~nm} \text {, view: axial }\end{array}$ \\
\hline Boron (B) & ICP-OES & $2 \%, 0.01$ & Leeman Labs Direct Reading Echelle ${ }^{3}$ & Wavelength: $249.678 \mathrm{~nm}$, view: axial \\
\hline $\begin{array}{l}\text { Aluminum } \\
\text { (Al) }\end{array}$ & $\begin{array}{l}\text { ICP-OES; } \\
\text { GFAAS }\end{array}$ & $\begin{array}{l}2 \%, 0.06 \\
5 \%, 0.001\end{array}$ & $\begin{array}{l}\text { Leeman Labs Direct Reading Echelle } \\
\text { Perkin-Elmer model } 4110 Z \mathrm{ZL}\end{array}$ & $\begin{array}{l}\text { Wavelength: } 308.215 \mathrm{~nm} \text {, view: axial; } \\
\text { Wavelength: } 309.3 \mathrm{~nm} \text {, modifier: } 15 \mu \mathrm{g} \\
\mathrm{Mg}\left(\mathrm{NO}_{3}\right)_{2} \text {, atomization temperature } 2,300^{\circ} \mathrm{C}\end{array}$ \\
\hline $\begin{array}{l}\text { Total dissolved } \\
\text { iron }(\mathrm{Fe}(\mathrm{T}))\end{array}$ & Colorimetry & $2 \%, 0.002$ & $\begin{array}{l}\text { Hewlett-Packard model } 8452 \mathrm{~A} \text { diode array } \\
\text { spectrometer with } 1-\text { and } 5-\mathrm{cm} \text { cells }\end{array}$ & $\begin{array}{l}\text { FerroZine method } \\
\text { (Stookey, 1970; To and others, 1999) }\end{array}$ \\
\hline $\begin{array}{l}\text { Ferrous iron } \\
(\mathrm{Fe}(\mathrm{II}))\end{array}$ & Colorimetry & $2 \%, 0.002$ & $\begin{array}{l}\text { Hewlett-Packard model } 8452 \mathrm{~A} \text { diode array } \\
\text { spectrometer with } 1-\text { and } 5-\mathrm{cm} \text { cells }\end{array}$ & $\begin{array}{l}\text { FerroZine method } \\
\text { (Stookey, 1970; To and others, 1999) }\end{array}$ \\
\hline $\begin{array}{l}\text { Manganese } \\
(\mathrm{Mn})\end{array}$ & ICP-OES & $3 \%, 0.001$ & Leeman Labs Direct Reading Echelle $^{3}$ & Wavelength: $257.610 \mathrm{~nm}$, view: axial \\
\hline Copper $(\mathrm{Cu})$ & $\begin{array}{l}\text { ICP-OES; } \\
\text { GFAAS }\end{array}$ & $\begin{array}{l}2 \%, 0.003 \\
3 \%, 0.0005\end{array}$ & $\begin{array}{l}\text { Leeman Labs Direct Reading Echelle } \\
\text { Perkin-Elmer model 4110ZL }\end{array}$ & $\begin{array}{l}\text { Wavelength: } 324.754 \mathrm{~nm} \text {, view: axial; } \\
\text { Wavelength: } 324.8 \mathrm{~nm} \text {, modifier: } 5 \mu \mathrm{g} \mathrm{Pd}+ \\
3 \mu \mathrm{gg}\left(\mathrm{NO}_{3}\right)_{2} \text {, atomization temperature: } \\
2,000^{\circ} \mathrm{C}\end{array}$ \\
\hline
\end{tabular}


Table 3. Analytical techniques, detection limits, typical precision, equipment used, and analytical method references-Continued.

\begin{tabular}{|c|c|c|c|c|}
\hline Constituent & Analytical Technique & $\begin{array}{l}\text { Typical RSD }{ }^{1} \\
\text { detection limit } \\
(\mathrm{mg} / \mathrm{L})\end{array}$ & Equipment Used & Comments or Reference(s) \\
\hline Zinc (Zn) & $\begin{array}{l}\text { ICP-OES; } \\
\text { GFAAS }\end{array}$ & $\begin{array}{l}2 \%, 0.004 \\
3 \%, 0.0005\end{array}$ & $\begin{array}{l}\text { Leeman Labs Direct Reading Echelle }{ }^{3} \text {; } \\
\text { Perkin-Elmer model 4110ZL }\end{array}$ & $\begin{array}{l}\text { Wavelength: } 213.856 \mathrm{~nm} \text {, view: axial; } \\
\text { Wavelength: } 213.9 \mathrm{~nm} \text {, modifier: } 5 \mu \mathrm{g} \\
\mathrm{Mg}\left(\mathrm{NO}_{3}\right)_{2} \text {, atomization temperature } \\
1,800^{\circ} \mathrm{C}\end{array}$ \\
\hline Cadmium (Cd) & $\begin{array}{l}\text { ICP-OES; } \\
\text { GFAAS }\end{array}$ & $\begin{array}{l}2 \%, 0.001 \\
5 \%, 0.0001\end{array}$ & $\begin{array}{l}\text { Leeman Labs Direct Reading Echelle }{ }^{3} \text {; } \\
\text { Perkin-Elmer model 4110ZL }\end{array}$ & $\begin{array}{l}\text { Wavelength: } 214.438 \mathrm{~nm} \text {, view: axial; } \\
\text { Wavelength: } 228.8 \mathrm{~nm} \text {, modifier: } 50 \mu \mathrm{g} \\
\mathrm{PO}_{4}+3 \mu \mathrm{g} \mathrm{Mg}\left(\mathrm{NO}_{3}\right)_{2} \text {, atomization } \\
\text { temperature: } 1,500^{\circ} \mathrm{C}\end{array}$ \\
\hline $\begin{array}{l}\text { Chromium } \\
(\mathrm{Cr})\end{array}$ & GFAAS & $5 \%, 0.0005$ & Perkin-Elmer model 4110ZL & $\begin{array}{l}\text { Wavelength: } 357.9 \mathrm{~nm} \text {, modifier: } 15 \mu \mathrm{g} \\
\mathrm{Mg}\left(\mathrm{NO}_{3}\right)_{2} \text {, atomization temperature: } \\
2,300^{\circ} \mathrm{C}\end{array}$ \\
\hline Cobalt (Co) & $\begin{array}{l}\text { ICP-OES; } \\
\text { GFAAS }\end{array}$ & $\begin{array}{l}2 \%, 0.004 \\
5 \%, 0.0007\end{array}$ & $\begin{array}{l}\text { Leeman Labs Direct Reading Echelle }{ }^{3} \text {; } \\
\text { Perkin-Elmer model 4110ZL }\end{array}$ & $\begin{array}{l}\text { Wavelength: } 228.616 \mathrm{~nm} \text {, view: axial; } \\
\text { Wavelength: } 242.5 \mathrm{~nm} \text {, modifier: } 15 \mu \mathrm{g} \\
\mathrm{Mg}\left(\mathrm{NO}_{3}\right)_{2} \text {, atomization temperature: } \\
2,400^{\circ} \mathrm{C}\end{array}$ \\
\hline $\begin{array}{l}\text { Total dissolved } \\
\text { mercury }(\mathrm{Hg}(\mathrm{T}))\end{array}$ & CVAFS & $2 \%, 0.4 \mathrm{ng} / \mathrm{L}$ & $\begin{array}{l}\text { PS Analytical, model Galahad, direct cold- } \\
\text { vapor atomic fluorescence spectrometry }\end{array}$ & $\begin{array}{l}\text { Taylor and others (1997), Roth and others } \\
\text { (2001) }\end{array}$ \\
\hline $\begin{array}{l}\text { Methylmercury } \\
\left(\mathrm{CH}_{3} \mathrm{Hg}\right)\end{array}$ & CVAFS & $2 \%, 0.04 \mathrm{ng} / \mathrm{L}$ & Tekran Model 2500 CVAFS mercury detector & $\begin{array}{l}\text { Distillation/ethylation/gas-phase separation } \\
\text { method (Horvat and others, 1993; DeWild } \\
\text { and others, 2002) }\end{array}$ \\
\hline Nickel (Ni) & $\begin{array}{l}\text { ICP-OES (2003); } \\
\text { GFAAS (2004-2005) }\end{array}$ & $\begin{array}{l}2 \%, 0.002 \\
3 \%, 0.0005\end{array}$ & $\begin{array}{l}\text { Leeman Labs Direct Reading Echelle }{ }^{3} \text {; } \\
\text { Perkin-Elmer model 4110ZL }\end{array}$ & $\begin{array}{l}\text { Wavelength: } 231.604 \mathrm{~nm} \text {, view: axial; } \\
\text { Wavelength: } 232.0 \mathrm{~nm} \text {, atomization } \\
\text { temperature: } 2,300^{\circ} \mathrm{C}\end{array}$ \\
\hline Lead $(\mathrm{Pb})$ & $\begin{array}{l}\text { ICP-OES; } \\
\text { GFAAS }\end{array}$ & $\begin{array}{l}5 \%, 0.008 \\
5 \%, 0.0008\end{array}$ & $\begin{array}{l}\text { Leeman Labs Direct Reading Echelle }{ }^{3} \text {; } \\
\text { Perkin-Elmer model 4110ZL }\end{array}$ & $\begin{array}{l}\text { Wavelength: } 220.353 \mathrm{~nm} \text {, view: axial; } \\
\text { Wavelength: } 283.3 \mathrm{~nm} \text {, modifier: } 50 \mu \mathrm{g} \\
\mathrm{PO}_{4}+3 \mu \mathrm{g} \mathrm{Mg}\left(\mathrm{NO}_{3}\right)_{2} \text {, atomization } \\
\text { temperature: } 1,600^{\circ} \mathrm{C}\end{array}$ \\
\hline Beryllium $(\mathrm{Be})$ & ICP-OES & $2 \%, 0.001$ & Leeman Labs Direct Reading Echelle ${ }^{3}$ & Wavelength: $313.042 \mathrm{~nm}$, view: axial \\
\hline Vanadium $(\mathrm{V})$ & ICP-OES & $3 \%, 0.005$ & Leeman Labs Direct Reading Echelle ${ }^{3}$ & Wavelength: $292.401 \mathrm{~nm}$, view: axial \\
\hline $\begin{array}{l}\text { Molybdenum } \\
\text { (Mo) }\end{array}$ & ICP-OES & $3 \%, 0.007$ & Leeman Labs Direct Reading Echelle ${ }^{3}$ & Wavelength: $277.540 \mathrm{~nm}$, view: axial \\
\hline
\end{tabular}


Table 3. Analytical techniques, detection limits, typical precision, equipment used, and analytical method references-Continued.

\begin{tabular}{|c|c|c|c|c|}
\hline Constituent & Analytical Technique & $\begin{array}{l}\text { Typical RSD1, } \\
\text { detection limit } \\
(\mathrm{mg} / \mathrm{L})\end{array}$ & Equipment Used & Comments or Reference(s) \\
\hline $\begin{array}{r}\text { Total dissolved } \\
\text { antimony }(\mathrm{Sb}(\mathrm{T}))\end{array}$ & GFAAS & $5 \%, 0.001$ & Perkin-Elmer model 4110ZL & $\begin{array}{l}\text { Wavelength: } 217.6 \mathrm{~nm} \text {, modifier: } 5 \mu \mathrm{g} \mathrm{Pd}+ \\
3 \mu \mathrm{g} \mathrm{Mg}\left(\mathrm{NO}_{3}\right)_{2} \text {, atomization temperature: } \\
1,900^{\circ} \mathrm{C}\end{array}$ \\
\hline $\begin{array}{l}\text { Antimonite } \\
(\mathrm{Sb}(\mathrm{III}))\end{array}$ & HGAAS & $3 \%, 0.0005$ & $\begin{array}{l}\text { Perkin-Elmer AAnalyst } 300 \text { atomic absorption } \\
\text { spectrometer with a FIAS-100 flow injection } \\
\text { analysis system, quartz cell, and furnace }\end{array}$ & Wavelength: $217.6 \mathrm{~nm}$ \\
\hline Selenium $(\mathrm{Se})$ & $\begin{array}{l}\text { ICP-OES; } \\
\text { GFAAS }\end{array}$ & $\begin{array}{l}5 \%, 0.04 \\
5 \%, 0.001\end{array}$ & $\begin{array}{l}\text { Leeman Labs Direct Reading Echelle }{ }^{3} \text {; } \\
\text { Perkin-Elmer model 4110ZL }\end{array}$ & $\begin{array}{l}\text { Wavelength: } 196.026 \mathrm{~nm} \text {, view: axial; } \\
\text { Wavelength: } 196.0 \mathrm{~nm} \text {, modifier: } 5 \mu \mathrm{g} \mathrm{Pd}+ \\
3 \mu \mathrm{ggg}\left(\mathrm{NO}_{3}\right)_{2} \text {, atomization temperature: } \\
2,100^{\circ} \mathrm{C}\end{array}$ \\
\hline $\begin{array}{l}\text { Total dissolved } \\
\text { arsenic }(\mathrm{As}(\mathrm{T}))\end{array}$ & $\begin{array}{l}\text { ICP-OES; } \\
\text { HGAAS }\end{array}$ & $\begin{array}{l}5 \%, 0.04 \\
2 \%, 0.0001\end{array}$ & $\begin{array}{l}\text { Leeman Labs Direct Reading Echelle } \\
\text { Perkin-Elmer AAnalyst } 300 \text { atomic absorption } \\
\text { spectrometer with a FIAS-100 flow injection } \\
\text { analysis system, quartz cell, and furnace }\end{array}$ & $\begin{array}{l}\text { Wavelength: } 188.977 \mathrm{~nm} \text {, view: axial; Pre- } \\
\text { reduction of As(V) using KI + ascorbic acid } \\
+\mathrm{HCl}(\mathrm{McCleskey} \text { and others, 2003) }\end{array}$ \\
\hline $\begin{array}{l}\text { Arsenite } \\
(\text { As(III)) }\end{array}$ & HGAAS & $5 \%, 0.001$ & $\begin{array}{l}\text { Perkin-Elmer AAnalyst } 300 \text { atomic absorption } \\
\text { spectrometer with a FIAS-100 flow injection } \\
\text { analysis system, quartz cell, and furnace }\end{array}$ & (McCleskey and others, 2003) \\
\hline Deuterium $(\delta \mathrm{D})$ & MS & 1 per mil $^{2}$ & V.G. Micromass model 602 mass spectrometer & $\begin{array}{l}\text { Standardization against VSMOW }(\delta \mathrm{D}=0 \\
\text { per mil) and SLAP }(\delta \mathrm{D}=-428 \text { per mil }) \\
(\text { Coplen and others, } 1991)\end{array}$ \\
\hline Oxygen $\left(\delta^{18} \mathrm{O}\right)$ & MS & 0.1 per mil ${ }^{2}$ & DuPont model 21-491 mass spectrometer & $\begin{array}{l}\text { Standardization against VSMOW }\left(\delta^{18} \mathrm{O}=0\right. \\
\text { per mil) and SLAP }\left(\delta^{18} \mathrm{O}=-55.5 \text { per mil) }\right. \\
\text { (Epstein and Mayeda, 1953) }\end{array}$ \\
\hline $\begin{array}{l}\text { Dissolved } \\
\text { organic carbon } \\
\text { (DOC) }\end{array}$ & TOC & $2 \%, 0.1$ & $\begin{array}{l}\text { Oceanography International Model } 700 \text { TOC } \\
\text { Analyzer }\end{array}$ & $\begin{array}{l}\text { Wet oxidation method } \\
\text { (Aiken, 1992) }\end{array}$ \\
\hline
\end{tabular}

${ }^{1}$ relative standard deviation expressed in percent $(100 \mathrm{x}$ standard deviation $\div$ mean $)$

${ }^{2}$ precision or range, rather than RSD

${ }^{3}$ dual view, sequential multi-element, inductively coupled plasma spectrometer. Hildebrand grid nebulizer and glass Scott spray chamber 


\section{Sulfide Determinations}

Three alternative methods were used to measure sulfide. Two methods were done on $\square$ site using colorimetry and a third method was done off-site on a preserved sample. For the first method, sample water was transferred into a measuring cuvette, and then color reagents were added. For the second method, sample water was transferred into a measuring cuvette to which color reagents and, for diluted samples, de-ionized water, had been added previously. After waiting for color development, the sample absorbance and temperature of the solution were measured. Dissolved sulfide concentration was measured on $\llbracket$ site using a battery-operated, portable ultraviolet-visible spectrophotometer and the methylene-blue method (Hach Company, 1992; equivalent to Standard Method 4500-S ${ }^{2-}$ D for wastewater; APHA, 1985). To minimize the inclusion of atmospheric air, samples for sulfide determination were collected directly from the outflow of a thoroughly equilibrated $142-\mathrm{mm}$ plastic filter with a $0.1-\mu \mathrm{m}$ membrane filter. When dilutions were prepared, sample water was filtered continuously into an overflowing $25-\mathrm{mL}$ polyethylene graduated cylinder from which aliquots were pipetted into de-ionized water in the measuring cuvette. For the third method, 15-mL Sulfide Anti-Oxidant Reagent (Sodium hydroxide-EDTA-ascorbic acid solution; Thermo-Orion \#941609) was placed in a 30-mL polyethylene bottle followed by $15 \mathrm{~mL}$ of filtered sample water. Sulfide concentrations were determined within 24 hours by ion-specific electrode.

Thiosulfate, if present at concentrations exceeding $10 \mathrm{mg} / \mathrm{L}$, can interfere with the colorimetric determination of sulfide by reacting with the color reagent and reducing or preventing color development (APHA, 1971). Although most $\mathrm{S}_{2} \mathrm{O}_{3}$ concentrations encountered in Yellowstone geothermal waters are less than this value, seven samples from four thermal features contained 12 to $42 \mathrm{mg} / \mathrm{L} \mathrm{S}_{2} \mathrm{O}_{3}$. Sulfide concentrations determined by colorimetry likely were underestimated in these cases.

Ball and others (2006) presented strong circumstantial evidence that suddenly reducing the $\mathrm{pH}$ of a hot sample before the introduction of color reagent creates a condition that favors rapid degassing of $\mathrm{H}_{2} \mathrm{~S}$ and consequent underestimation of sulfide concentration. When the Hach standard method was modified by mixing Hach reagents 1 and 2 in the cuvette before introducing the sample, the measured sulfide concentrations were marginally higher (fig. 14). Notwithstanding this increase in measured concentrations, the color reagent appears to degrade rapidly after mixing with reagent 1 ; therefore, the short but variable elapsed times between reagent mixing and sample addition may add an enormous imprecision component to the measurements. The added step of cooling the hot sample by diluting 2.5 to 25 times (data not shown) does not appear to fully mitigate this source of error.

Sulfide concentrations measured with the ion-selective electrode were consistently higher than those measured on $\square$ site by colorimetry (fig. 15). These results strongly suggest that (1) the sulfide in samples issuing from the subsurface at elevated temperature is unstable with respect to oxidation and $\mathrm{H}_{2} \mathrm{~S}$ degassing and must be handled with care and dispatch to obtain accurate concentration estimates, and (2) the methylene-blue colorimetric method, regardless of any modifications made in an attempt to improve its accuracy, is not suitable for use in waters having temperatures exceeding ambient. Our results to date indicate that stabilizing the sulfide in geothermal water by immediately combining the hot sample with an alkaline solution containing sulfide anti-oxidant buffer (SAOB) oxidation preventative offers the best option for obtaining accurate estimates of sulfide concentrations. 


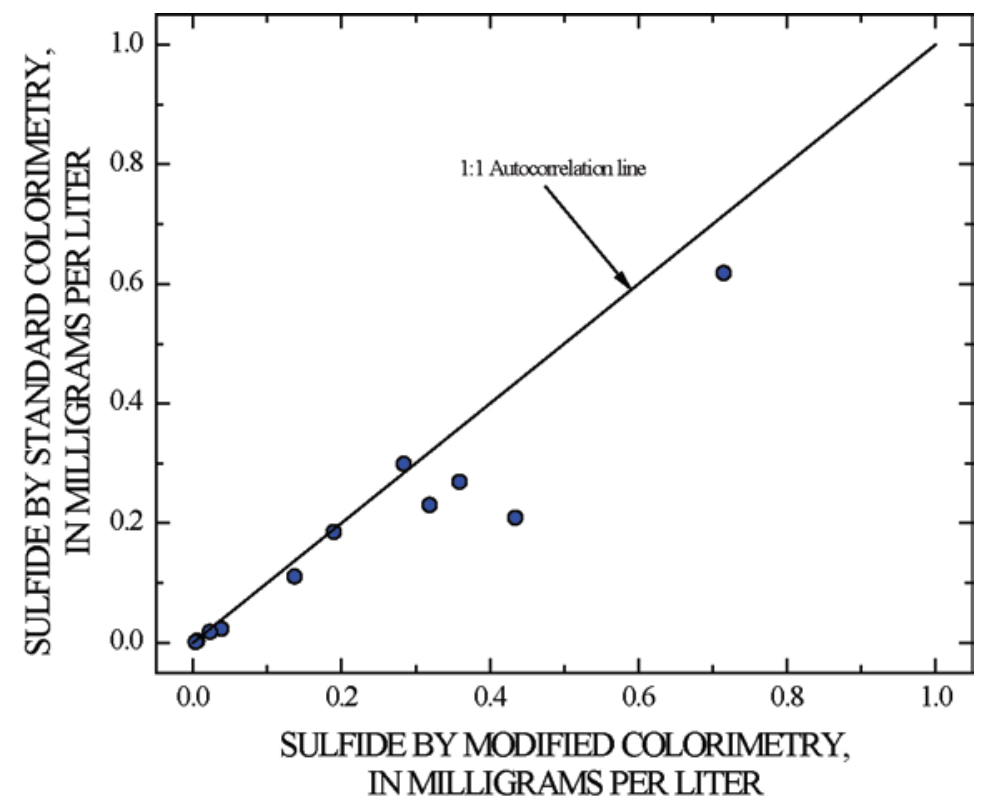

Figure 14. Comparison of results of sulfide determinations using standard and modified methylene-blue colorimetric methods.

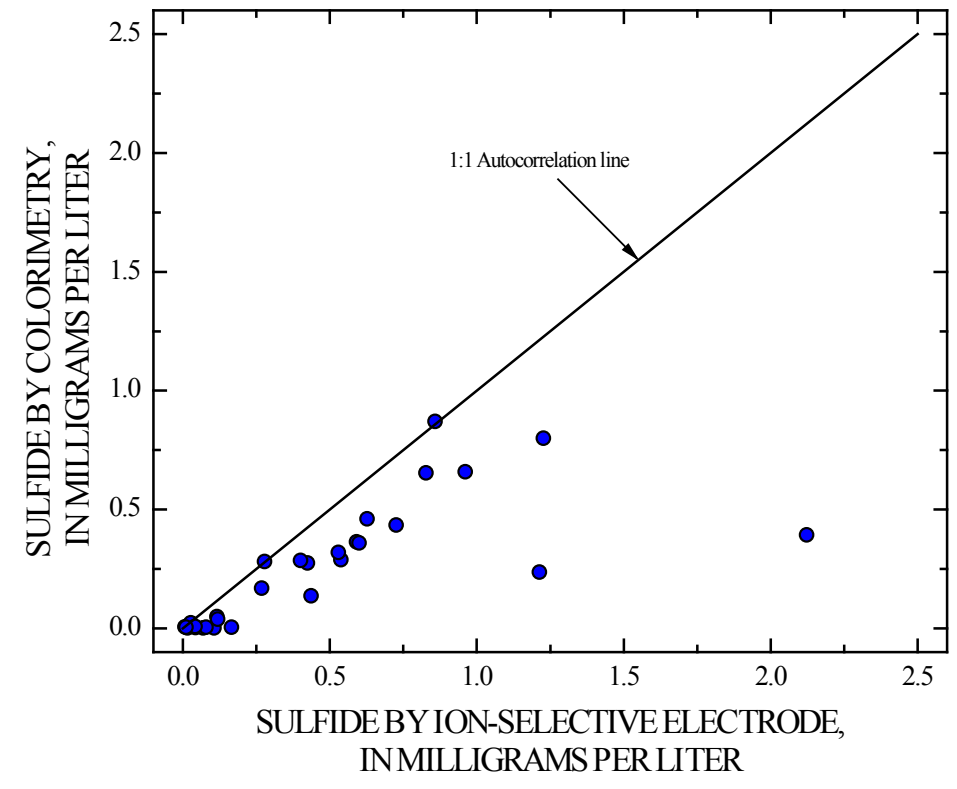

Figure 15. Comparison of results of sulfide determinations using the ion-selective electrode method and best values from the methylene-blue colorimetric method. 


\section{Major Cation and Trace Metal Determinations}

Concentrations of major cations and trace metals were determined using inductively coupled plasma-optical emission spectrometry (ICP-OES) at the USGS laboratory in Boulder, Colorado. Major cations were determined using the radial plasma viewing orientation while the axial plasma viewing orientation was used for trace metals. A cesium chloride ionization buffer was added to the samples for ICP-OES measurement of Li and $\mathrm{K}$ concentrations. Wavelengths and plasma viewing orientations are specified in table 3 .

Concentrations of $\mathrm{Al}, \mathrm{Sb}, \mathrm{Cd}, \mathrm{Cr}, \mathrm{Co}, \mathrm{Cu}, \mathrm{Ni}, \mathrm{Pb}, \mathrm{Se}$, and $\mathrm{Zn}$ were measured using graphite-furnace atomic absorption spectrometry (GFAAS) with a transversely heated graphite atomizer (THGA) and Zeeman-effect background correction. Wavelengths, atomization temperatures, and matrix modifiers used are listed in table 3.

Hydride-generation atomic absorption spectrometry was used to measure total dissolved arsenic $(\mathrm{As}(\mathrm{T}))$, dissolved arsenite (As(III)), and total dissolved antimony $(\mathrm{Sb}(\mathrm{T}))$ concentrations in HCl-acidified samples. A flow-injection analysis system (FIAS) was used to generate arsine (McCleskey and others, 2003) or stibine. Total dissolved iron ( $\mathrm{Fe}(\mathrm{T}))$ and ferrous iron (Fe(II)) concentrations were determined in samples preserved with $\mathrm{HCl}$ using a modification of the FerroZine colorimetric method (Stookey, 1970; Gibbs, 1976; To and others, 1999).

Samples were analyzed for total dissolved $\mathrm{Hg}$ at the USGS laboratory in Boulder, Colorado. Samples were analyzed for methylmercury $\left(\mathrm{CH}_{3} \mathrm{Hg}\right)$ at the USGS Mercury Research Laboratory in Middleton, Wisconsin. Total dissolved $\mathrm{Hg}$ was determined by direct cold-vapor atomic fluorescence spectroscopy (CVAFS) following oxidation with chromate, reduction by $\mathrm{SnCl}_{2}$, and purge and trap of the evolved zero-valent $\mathrm{Hg}$ onto gold-coated glass-bead columns (Taylor and others, 1997; Roth and others, 2001). The method detection limit was 0.4 nanograms per liter $(\mathrm{ng} / \mathrm{L})$.

Methylmercury concentrations were determined using the distillation/ethylation/gasphase separation method of Olson and DeWild (1999) and detection by CVAFS (Horvat and others, 1993). The method detection limit was $0.04 \mathrm{ng} / \mathrm{L}$. All samples were analyzed in duplicate and reanalyzed if the relative percent difference was greater than 10 percent. Analytical precision was about $\pm 0.02 \mathrm{ng} / \mathrm{L}$ (DeWild and others, 2002).

\section{Major Anion Determinations}

Concentrations of $\mathrm{Br}, \mathrm{Cl}, \mathrm{F}$, and $\mathrm{SO}_{4}$ were determined at the USGS laboratory in Boulder, Colorado, by ion chromatography (IC) (Brinton and others, 1995). Fluoride concentrations were determined using an ion-selective electrode (ISE). Samples for F determination by ISE were mixed 1:1 with a total ionic strength adjustment buffer (TISAB III) (Barnard and Nordstrom, 1980). Alkalinity was determined in the mobile field laboratory or at the USGS laboratory in Boulder, Colorado, by automated titration using standardized $\mathrm{H}_{2} \mathrm{SO}_{4}$ (Barringer and Johnsson, 1996).

\section{Nutrient and Dissolved Organic Carbon Determinations}

Nutrient concentrations were determined at the USGS laboratory in Boulder, Colorado. Ammonium concentrations were determined by IC with $50 \mathrm{mN} \mathrm{H}_{2} \mathrm{SO}_{4}$ eluent. Nitrate was measured by IC with a $3.5 \mathrm{mM} \mathrm{NaHCO}_{3}+1.0 \mathrm{mM} \mathrm{Na}_{2} \mathrm{CO}_{3}$ eluent (Brinton and others, 1995).

Dissolved organic carbon concentrations were measured using the wet oxidation method (Aiken, 1992) with Oceanography International Model 700 TOC Analyzer. Potassium 
biphthalate was used to calibrate the instrument, and sodium benzoate was used as a different organic carbon source to check the calibration. Analyses of inorganic carbon, $\mathrm{HCO}_{3}$, alongside the samples provided a daily verification that the acid valve was functioning properly.

\section{Water Isotope Determinations}

Hydrogen and oxygen isotope ratios were determined at the USGS Reston Stable Isotope Laboratory in Reston, Virginia (http://isotopes.usgs.gov/). Hydrogen isotope ratios were determined using a hydrogen equilibration technique (Coplen and others, 1991; Revesz and Coplen, 2008). Oxygen isotope ratios were determined using the $\mathrm{CO}_{2}$ equilibration technique of Epstein and Mayeda (1953), which has been automated by Revesz and Coplen (2008). The isotopic concentration is reported in "delta notation," which compares the isotope ratio of a sample to that of a reference standard. For the example of ${ }^{18} \mathrm{O} /{ }^{16} \mathrm{O}$ ratios, delta notation is:

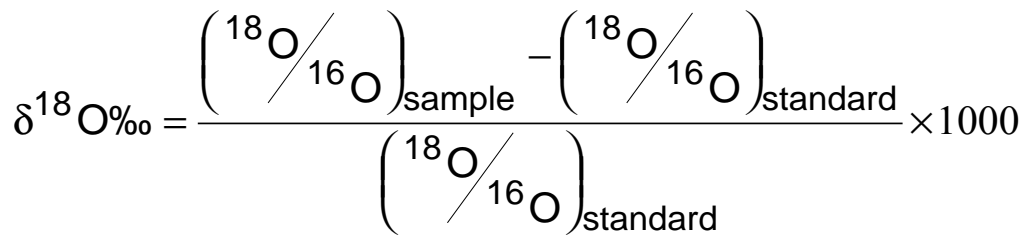

where $\%$ is per mil, which is equivalent to parts per thousand.

Oxygen and hydrogen isotopic results are reported relative to the standard VSMOW (Vienna Standard Mean Ocean Water) and normalized (Coplen, 1994) on scales such that the oxygen and hydrogen isotopic values of SLAP (Standard Light Antarctic Precipitation) are -55.5 per mil and -428 per mil, respectively.

\section{Acidity Determinations}

Total acidity was determined in the mobile field laboratory or at the USGS laboratory in Boulder, Colorado, by titrating samples having $\mathrm{pH}$ less than 4 to $\mathrm{pH}$ greater than 7 using an autotitrator and standardized sodium hydroxide $(\mathrm{NaOH})$ solution. The $\mathrm{NaOH}$ titrant $(0.01-0.05$ $\mathrm{M}$ ) was standardized daily by titrating a known quantity of potassium hydrogen phthalate $\left(\mathrm{KHC}_{8} \mathrm{H}_{4} \mathrm{O}_{4}\right)$. The titrator was programmed for 50 - to $100-\mu \mathrm{L}$ constant-volume additions or constant change in millivolts $(\mathrm{mV})$, typically $10 \mathrm{mV}$ per addition. Equivalence points were determined using a modified Gran's function (Gran, 1952; Barringer and Johnsson, 1996):

$$
\mathrm{F}_{\text {acid }}=\left(\mathrm{v}_{0}+\mathrm{v}_{\mathrm{NaOH}}\right) \times 10^{-\mathrm{pOH}}
$$

where $\mathrm{F}_{\text {acid }}=$ the Gran function, $\mathrm{v}_{0}=$ sample volume, $\mathrm{v}_{\mathrm{NaOH}}=$ volume of $\mathrm{NaOH}$ titrant added, and $\mathrm{pOH}=14$ minus $\mathrm{pH}$.

The principal components contributing to acidity were discussed by Ball and others (2006). Free $\mathrm{H}^{+}$was derived from the total acidity by subtracting the hydrogen ions produced by 
hydrolysis of $\mathrm{SO}_{4}, \mathrm{Fe}, \mathrm{Al}, \mathrm{As}(\mathrm{V})$, and $\mathrm{F}$, as calculated by PHREEQCi, a graphical-user-interface version (Charlton and others, 1997) of program PHREEQC (Parkhurst and Appelo, 1999) incorporating the WATEQ4F database, as follows:

$$
\begin{aligned}
& \text { Acidity }_{\mathrm{SO}_{4}}=\mathrm{HSO}_{4}^{-} \\
& \text {Acidity }_{\mathrm{Fe}}=3\left(\mathrm{Fe}^{\mathrm{III}}(\mathrm{tot})-\mathrm{Fe}(\mathrm{OH})_{2}^{+}\right)+2 \mathrm{FeOH}{ }^{2+}+\mathrm{Fe}(\mathrm{OH})_{2}^{+}+2 \mathrm{Fe}^{\mathrm{II}} \text { (tot) } \\
& \text { Acidity }_{\mathrm{Al}}=3\left(\mathrm{Al}(\mathrm{tot})-\mathrm{AlOH}^{2+}-\mathrm{Al}(\mathrm{OH})_{2}^{+}\right)+2 \mathrm{AlOH}^{2+}+\mathrm{Al}(\mathrm{OH})_{2}^{+} \\
& \text {Acidity }_{\mathrm{As}^{+}}=2\left(\mathrm{As}^{\mathrm{V}}(\mathrm{tot})-\mathrm{H}_{2} \mathrm{AsO}_{4}^{-}\right)+\mathrm{H}_{2} \mathrm{AsO}_{4}^{-} \\
& \text {Acidity }_{\mathrm{F}}=\mathrm{HF}^{0} \\
& \text { Acidity }_{\mathrm{H}^{+}}=\mathrm{Acidity}_{\mathrm{Total}^{-}} \text {-Acidity }
\end{aligned}
$$

Concentrations for the above equations are expressed in moles per kilogram of water. Sample $\mathrm{pH}$ from the acidity titration (acidity $\mathrm{pH}$ ) was calculated by computing the product of the $\mathrm{H}^{+}$activity coefficient (calculated by PHREEQCi) and the free $\mathrm{H}^{+}$molality (eq. 8, calculated using a spreadsheet) and computing the negative common logarithm of the resulting activity. This $\mathrm{pH}$ value was refined by repeating the PHREEQCi calculation and varying the input $\mathrm{pH}$ until the $\mathrm{pH}$ calculated from the PHREEQCi speciation was equal to the input $\mathrm{pH}$. A flow-chart illustrating the process for refining the acidity $\mathrm{pH}$ is shown in figure 16. 


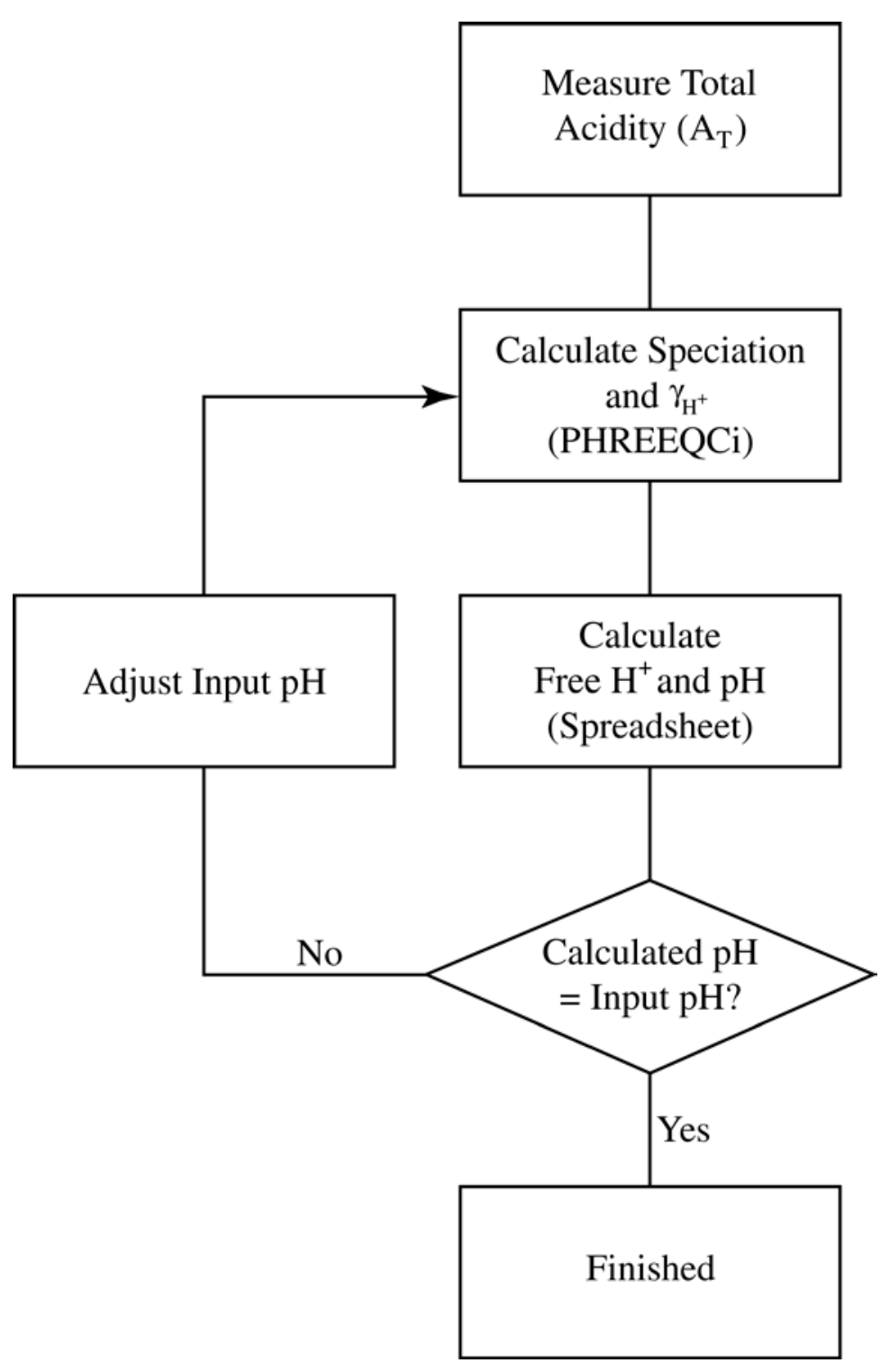

Figure 16. Flow chart illustrating the process for refining the acidity $\mathrm{pH}$ value.

\section{Revised pH Measurements}

Accurate measurement of $\mathrm{pH}$ is of primary importance for interpretation of aqueous chemical speciation. The free hydrogen ion $\left(\mathrm{H}^{+}\right)$is usually the major cation in samples with $\mathrm{pH}$ less than 2.5 in geothermal waters (Ball and others, 2002), is important in controlling geochemical reactions, and is critical in calculating the charge imbalance (C.I.) for waters with $\mathrm{pH}<3$. For the subset of 47 samples with $\mathrm{pH}<4, \mathrm{pH}$ values determined using four different techniques are shown in table 4: (1) $\mathrm{pH}$ measured in the field; (2) $\mathrm{pH}$ measured at the USGS laboratory in Boulder, Colorado; (3) acidity $\mathrm{pH}$ (calculated as discussed in the previous section); and (4) $\mathrm{pH}$ calculated by adjusting the concentration of $\mathrm{H}^{+}$to yield a speciated C.I. of zero. 
Comparison of $\mathrm{pH}$ values from the four sources allows evaluation of the measurements and estimation of more accurate $\mathrm{pH}$ values.

A flow chart showing the $\mathrm{pH}$ selection process is shown in figure 17. Field $\mathrm{pH}$ was considered to be the most accurate because $\mathrm{pH}$ measurements made in the laboratory may be biased from temperature changes and hydrolysis reactions, and the acidity $\mathrm{pH}$ calculation relies on measurements of $\mathrm{SO}_{4}, \mathrm{Fe}, \mathrm{Al}, \mathrm{As}$, and $\mathrm{F}$, all of which are subject to analytical uncertainties. In this report, $\mathrm{pH}$ calculated by using the concentration of $\mathrm{H}^{+}$to adjust the speciated C.I. to zero was used only as a reference because C.I. $\mathrm{pH}$ relies on measurements of many constituents.

Differences between field and laboratory $\mathrm{pH}$ values were calculated by subtracting the laboratory $\mathrm{pH}$ value from the field $\mathrm{pH}$ value. Difference values were found to be greatest for samples with initial $\mathrm{pH}$ values between 5 and 8 (fig. 18). Laboratory $\mathrm{pH}$ measurements typically are made at about $22^{\circ} \mathrm{C}$ which can be as much as $70^{\circ} \mathrm{C}$ less than the temperature at which the sample $\mathrm{pH}$ was measured in the field. Differences in $\mathrm{pH}$ values between field and laboratory measurements are a function of the changes in sample temperature and composition that occur between the time of collection and the time that laboratory $\mathrm{pH}$ values are determined. For samples with $\mathrm{pH}$ less than 4.5 , differences are greatest at $\mathrm{pH}$ greater than 3.0 and higher sample temperatures (fig. 19). For samples with $\mathrm{pH}$ greater than 4.5, many of the samples that had $\mathrm{pH}$ reductions most likely were poorly buffered, whereas samples that had $\mathrm{pH}$ increases likely were initially supersaturated with respect to atmospheric $\mathrm{CO}_{2}$. Thus, positive differences, indicating laboratory $\mathrm{pH}$ values that are less than field $\mathrm{pH}$ values, likely are the result of Fe oxidation and hydrolysis (Nordstrom and Alpers, 1999) or oxidation of reduced S species, whereas negative values result from degassing of $\mathrm{CO}_{2}$ (Nordstrom and others, 1990; fig. 20).

Charge imbalance variations also were found to be strong functions of temperature and $\mathrm{pH}$. When comparing C.I. for samples calculated using $\mathrm{pH}$ measured in the field to C.I. for the same samples calculated using $\mathrm{pH}$ measured in the laboratory, the C.I. difference is seen to be greatest for samples with the lowest $\mathrm{pH}$ values and high temperatures. Figure 21 shows the difference between C.I. calculated at $22^{\circ} \mathrm{C}$ and C.I. calculated at field temperature as a function of field temperature. This difference can be attributed to the hydrolysis of $\mathrm{SO}_{4}$, which is a function of both temperature and $\mathrm{pH}$ (Nordstrom and others, 2009) and possibly to other aspects of speciation such as the temperature dependence of activity coefficients.

Field $\mathrm{pH}$ was selected for all samples with $\mathrm{pH}$ greater than 3.5. For samples having a $\mathrm{pH}$ less than 3.5, field $\mathrm{pH}$ was selected unless the sample had a speciated C.I. greater than 10 percent using field $\mathrm{pH}$. For samples with $\mathrm{pH}$ less than 3.5 and speciated C.I. greater than 10 percent using field $\mathrm{pH}$, laboratory $\mathrm{pH}$ was selected if the speciated C.I. was less than 10 percent. For samples with $\mathrm{pH}$ less than 3.5 and speciated C.I. greater than 10 percent using both field and laboratory $\mathrm{pH}$, acidity $\mathrm{pH}$ was selected if the speciated C.I. was less than 10 percent. For samples with $\mathrm{pH}$ less than 3.5 and having field, laboratory, and acidity $\mathrm{pH}$ values that produced speciated C.I.s all greater than 10 percent, the $\mathrm{pH}$ that produced the lowest speciated C.I. was selected from among field, laboratory, and acidity $\mathrm{pH}$.

Using the process described above and illustrated in figure 17 for the subset of 47 samples, field $\mathrm{pH}$ was selected for 33 samples, laboratory $\mathrm{pH}$ was selected for 13 samples, and acidity $\mathrm{pH}$ was selected for one sample. Values of the selected $\mathrm{pH}$ are found in the tables of chemical data (tables 6-14) along with the speciated C.I. calculated using the selected $\mathrm{pH}$ and field temperature. 


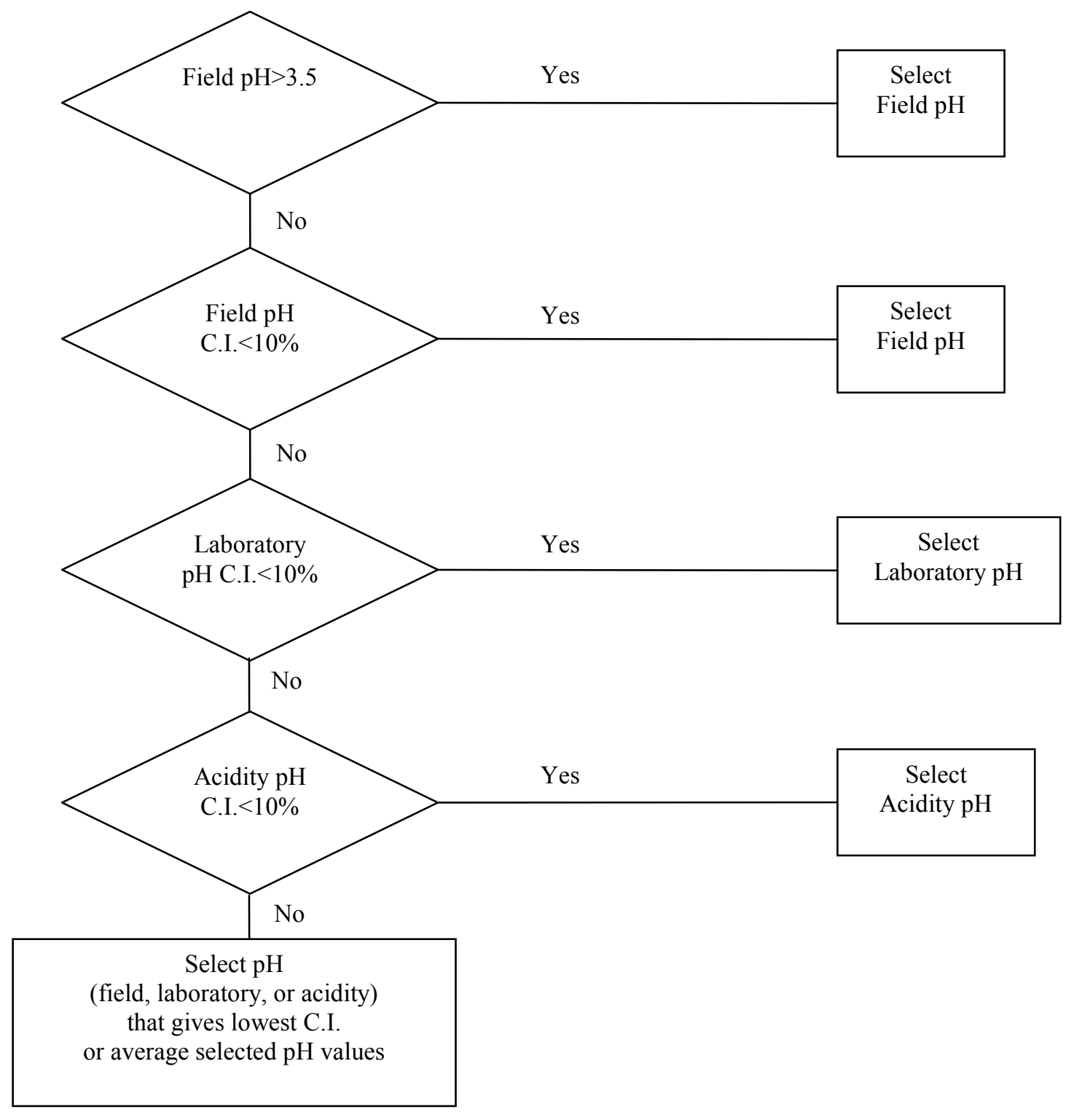

Figure 17. Flow chart showing the $\mathrm{pH}$ selection process. C.I., charge imbalance; >, greater than; <, less than. 


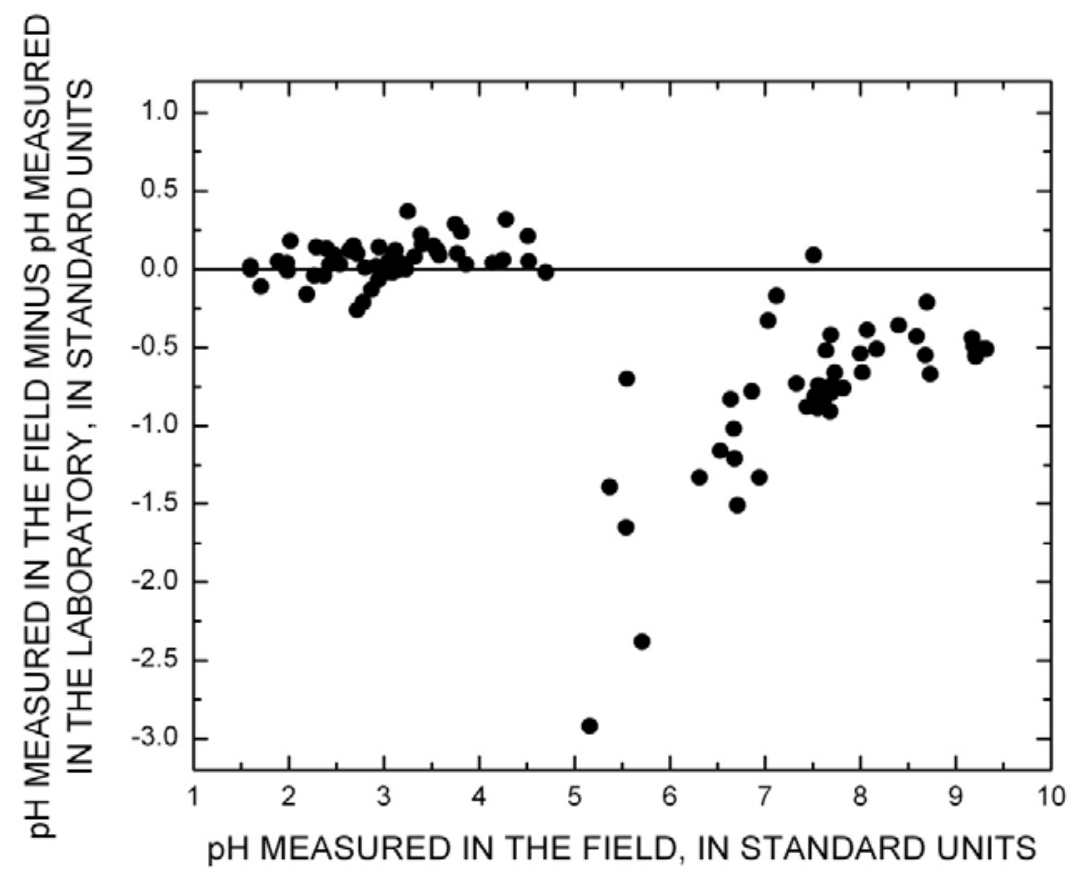

Figure 18. Difference between field and laboratory $\mathrm{pH}$ as a function of field $\mathrm{pH}$.

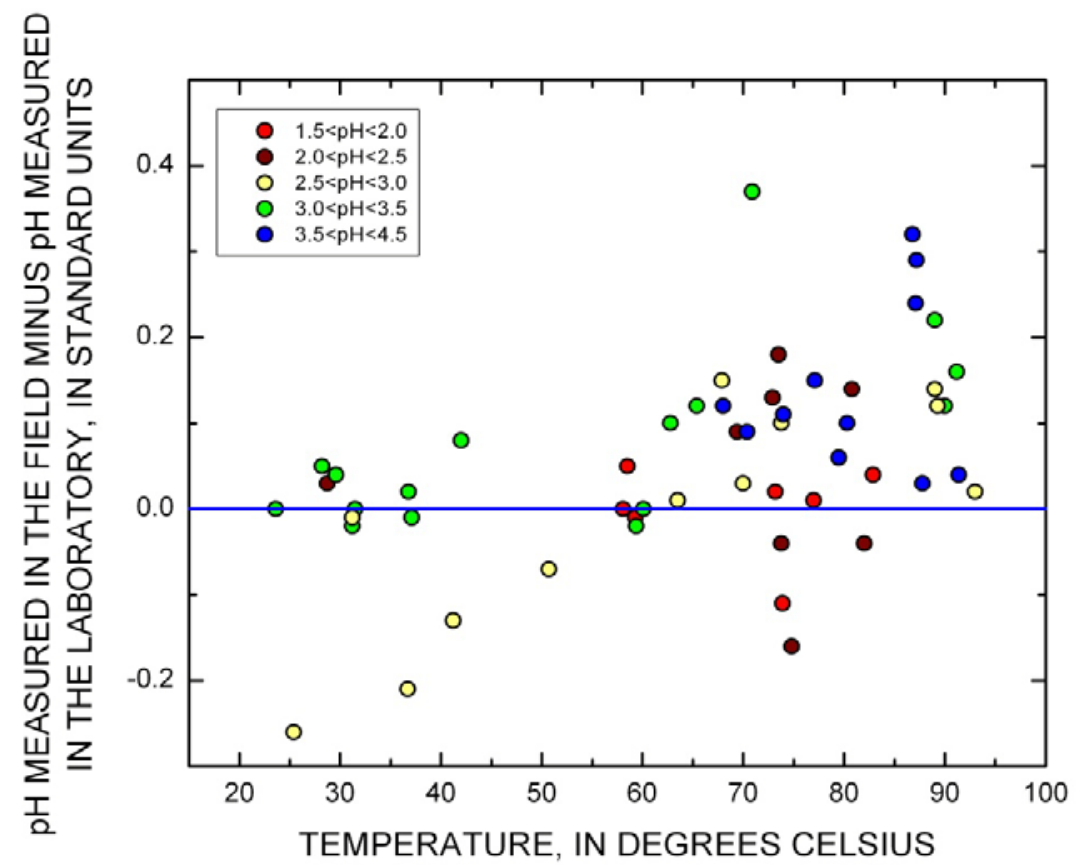

Figure 19. Difference between field and laboratory $\mathrm{pH}$ as a function of sample temperature, for samples with $\mathrm{pH}$ less than $(<) 4.5$. 


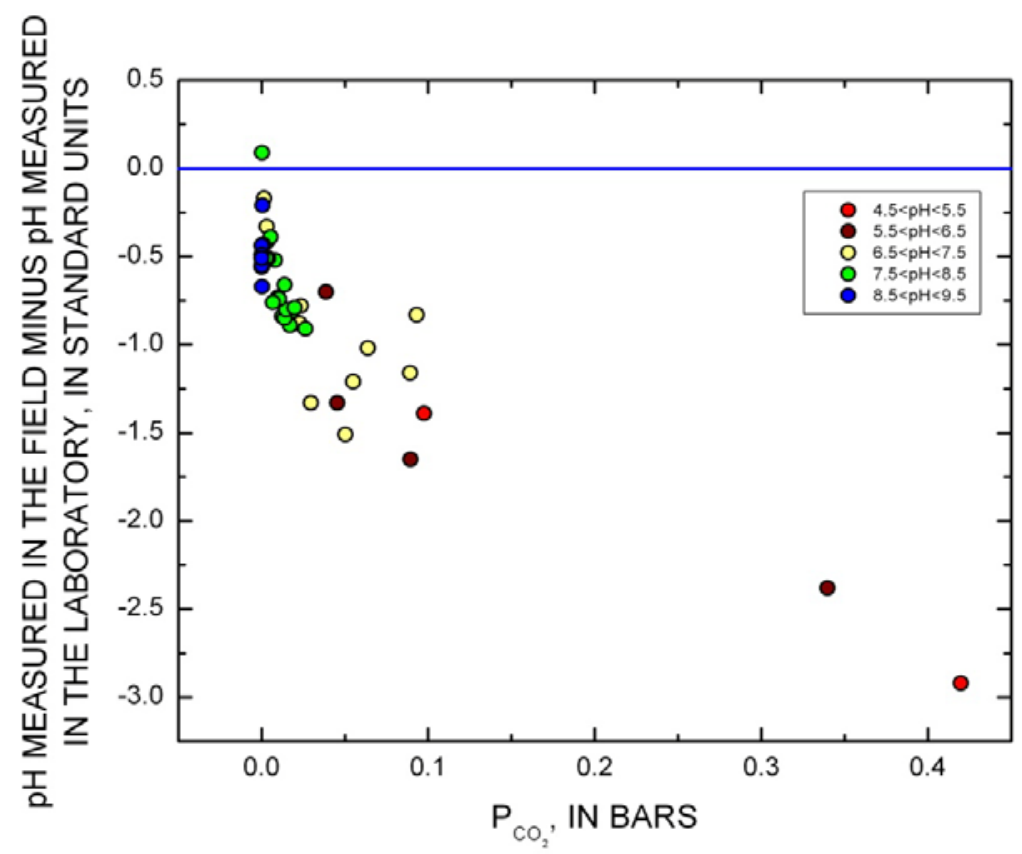

Figure 20. Difference between field and laboratory $\mathrm{pH}$ as a function of partial pressure of $\mathrm{CO}_{2}$.

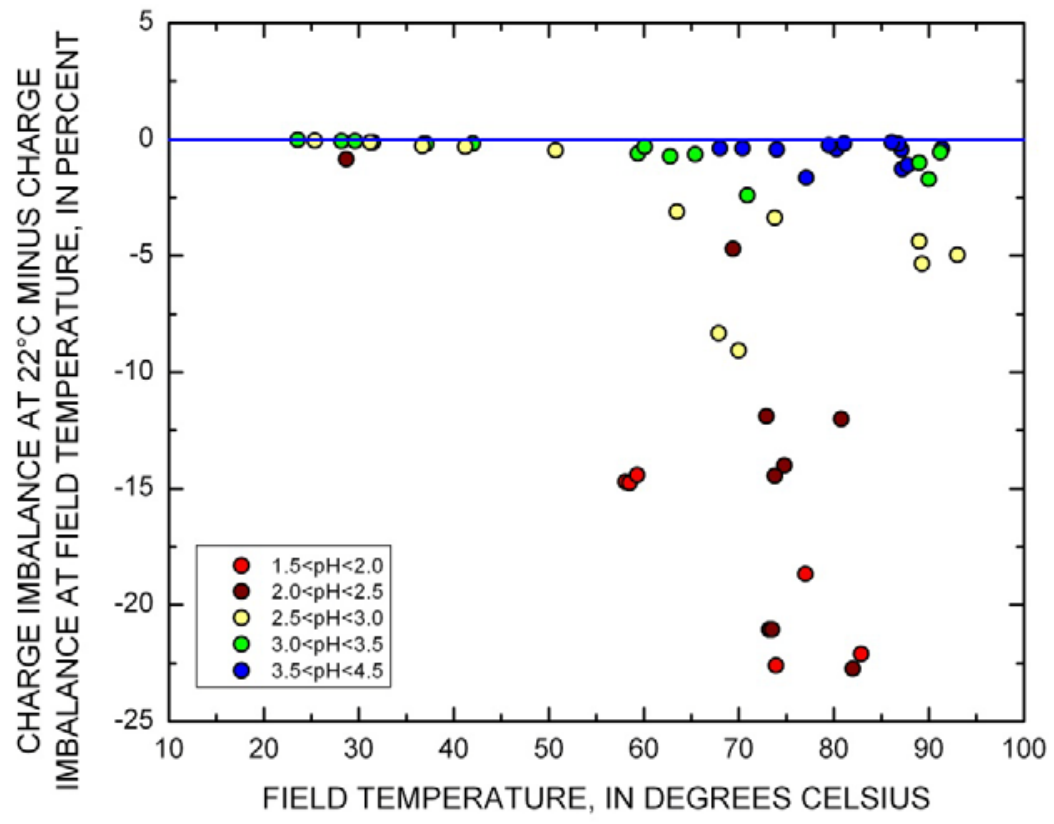

Figure 21. Difference between speciated charge imbalance at $22^{\circ} \mathrm{C}$ and speciated charge imbalance at field temperature in relation to field temperature. 
Table 4. Sample pH measured in the field and laboratory and calculated from acidity titration and speciated charge imbalance, for samples with pH less than 4.

\begin{tabular}{|c|c|c|c|c|c|}
\hline \multirow[b]{2}{*}{ Sampl e } & \multicolumn{5}{|c|}{ - - - - - - pH, in st andard uni ts - - - - - } \\
\hline & $\begin{array}{c}\text { Measur ed } \\
\text { In the } \\
\text { f i el d } \\
\end{array}$ & $\begin{array}{c}\text { Measur ed } \\
\text { In t he } \\
\text { I abor at or y }\end{array}$ & $\begin{array}{c}\text { Cal cul at ed } \\
\text { f rom aci di t y } \\
\text { ti trat i on }\end{array}$ & $\begin{array}{c}\text { Cal cul at ed } \\
\text { f r om char ge } \\
\text { i mbal ance }\end{array}$ & Sel ect ed \\
\hline $\begin{array}{l}\text { 06WA106, Gibbon River inflow from } \\
\text { Realgar Spring area }\end{array}$ & 2.99 & 3.00 & 2.95 & 2.78 & 2.99 \\
\hline 06WA107, Tantalus Creek at weir & 3.09 & 3.11 & 3.07 & 2.76 & 3.09 \\
\hline $\begin{array}{l}\text { 06WA109, Unnamed thermal feature near } \\
\text { Cinder Pool }\end{array}$ & 3.56 & 3.45 & 3.41 & 3.32 & 3.56 \\
\hline 06WA1 10, 'Orpiment Puddle 3" & 2.43 & 2.40 & 2.43 & 2.38 & 2.43 \\
\hline 06WA111, Crystal Spring - western side & 3.77 & 3.67 & 3.68 & 3.25 & 3.77 \\
\hline 06WA117, "Titanic Spring" & 3.56 & 3.44 & 3.42 & 3.06 & 3.56 \\
\hline 06WA120, The Gap southwest outflow & 3.08 & 3.06 & 3.06 & 3.24 & 3.08 \\
\hline 06WA121, The Gap northeast outflow & 3.32 & 3.24 & 3.21 & 2.78 & 3.32 \\
\hline 06WA126, "Succession Spring" & 3.12 & 3.00 & 2.97 & 2.88 & 3.12 \\
\hline $\begin{array}{l}\text { 06WA127, 'Succession Spring" overflow } \\
\text { channel }\end{array}$ & 3.02 & 3.04 & 3.01 & 2.83 & 3.02 \\
\hline $\begin{array}{l}\text { 06WA128, 'Succession Spring" overflow } \\
\text { channel }\end{array}$ & 2.94 & 3.01 & 2.98 & 2.79 & 2.94 \\
\hline $\begin{array}{l}\text { 06WA129, "Succession Spring" overflow } \\
\text { channel }\end{array}$ & 2.87 & 3.00 & 2.98 & 2.75 & 2.87 \\
\hline $\begin{array}{l}\text { 06WA130, 'Succession Spring" overflow } \\
\text { channel }\end{array}$ & 2.78 & 2.99 & 2.96 & 2.77 & 2.78 \\
\hline $\begin{array}{l}\text { 06WA133, Unnamed acid spring near } \\
\text { Perpetual Spouter }\end{array}$ & 2.95 & 2.81 & 2.95 & 2.84 & 2.95 \\
\hline $\begin{array}{l}\text { 06WA134, Tantalus Creek at Back Basin } \\
\text { exit }\end{array}$ & 3.13 & 3.14 & 3.07 & 2.88 & 3.13 \\
\hline 06WA139, Realgar Creek near mouth & 3.05 & 3.00 & 2.99 & 2.70 & 3.05 \\
\hline 06WA158, Tantalus Creek at weir & 3.02 & 3.02 & 2.99 & 2.78 & 3.02 \\
\hline 06WA164, Sulphur Caldron & 2.02 & 1.84 & 2.06 & 1.99 & 2.02 \\
\hline 06WA165, Turbulent Pool & 1.89 & 1.84 & 1.88 & 1.82 & 1.84 \\
\hline 06WA167, Washburn Inkpot \#3 & 3.25 & 2.88 & 3.24 & 2.63 & 2.88 \\
\hline 07WA105, "Persnickety Geyser" & 3.39 & 3.17 & 3.28 & 3.15 & 3.39 \\
\hline 07WA106, "Kaolin Spring" & 2.37 & 2.41 & 2.41 & 2.41 & 2.37 \\
\hline $\begin{array}{l}\text { 07WA107, Hot spring next to "Orpiment } \\
\text { Puddle 2" }\end{array}$ & 3.12 & 3.00 & 3.08 & 3.02 & 3.12 \\
\hline $\begin{array}{l}\text { 07WA108, Unnamed thermal feature, } \\
\text { Geyser Springs Group }\end{array}$ & 2.19 & 2.35 & 2.45 & 2.44 & 2.35 \\
\hline
\end{tabular}


Table 4. Sample $\mathrm{pH}$ m easured in the $\mathrm{f}$ ield and $\mathrm{I}$ aboratory a nd $\mathrm{c}$ alculated $\mathrm{f}$ rom ac idity $\mathrm{t}$ itration an $\mathrm{d}$ speciated charge imbalance, for samples with $\mathrm{pH}$ less than 4-Continued.

\begin{tabular}{|c|c|c|c|c|c|}
\hline \multirow[b]{2}{*}{ Sampl e } & \multicolumn{5}{|c|}{$-\ldots-\ldots$} \\
\hline & $\begin{array}{c}\text { Measur ed } \\
\text { In the } \\
\text { f i el d } \\
\end{array}$ & $\begin{array}{c}\text { Measur ed } \\
\text { In t he } \\
\text { I abor at or y }\end{array}$ & $\begin{array}{c}\text { Cal cul at ed } \\
\text { f rom aci di t y } \\
\text { ti trat i on }\end{array}$ & $\begin{array}{c}\text { Cal cul at ed } \\
\text { f r om char ge } \\
\text { i mbal ance }\end{array}$ & Sel ect ed \\
\hline 07WA109, Turbulent Pool & 1.99 & 2.00 & 2.02 & 1.93 & 1.99 \\
\hline 07WA1 10, Sulphur Caldron & 1.71 & 1.82 & 1.77 & 1.63 & 1.71 \\
\hline 07WA111, Sulphur Spring & 3.86 & 3.83 & 3.31 & 2.83 & 3.86 \\
\hline $\begin{array}{l}\text { 07WA1 } 12 \text {, Unnamed thermal feature, } \\
\text { Crater H ills area }\end{array}$ & 2.27 & 2.31 & 2.36 & 2.14 & 2.36 \\
\hline 07WA1 14, "Titanic Spring" & 3.10 & 3.00 & 3.06 & 2.87 & 3.10 \\
\hline 07WA115, "Lifeboat Spring" & 2.48 & 2.39 & 2.50 & 2.47 & 2.48 \\
\hline $\begin{array}{l}\text { 07WA145, Unnamed acid spring near } \\
\text { Perpetual Spouter }\end{array}$ & 2.92 & 2.90 & 2.97 & 2.83 & 2.92 \\
\hline 07WA146, Crystal Spring - western side & 1.98 & 1.94 & 2.03 & 1.92 & 1.94 \\
\hline 07WA147, Crystal Spring - eastern side & 1.97 & 1.96 & 2.06 & 1.81 & 1.96 \\
\hline 08WA105, Tantalus Creek at weir & 3.06 & 3.06 & 3.06 & 2.45 & 3.06 \\
\hline 08WA114, Sulphur Caldron & 1.60 & 1.58 & 1.97 & 1.54 & 1.58 \\
\hline 08WA1 15, Sulphur Spring & 3.75 & 3.46 & 3.28 & 2.66 & 3.46 \\
\hline $\begin{array}{l}\text { 08WA1 16, Unnamed acid pool near } \\
\text { Sulphur Spring }\end{array}$ & 2.72 & 2.62 & 2.76 & 2.32 & 2.62 \\
\hline $\begin{array}{l}\text { 08WA1 17, Unnamed acid pool near } \\
\text { "Bullseye Spring," Geyser Springs Group }\end{array}$ & 2.68 & 2.53 & 2.90 & 2.18 & 2.53 \\
\hline 08WA1 19, Deep circular pool & 1.60 & 1.60 & 1.98 & 1.42 & 1.60 \\
\hline 08WA120, Crystal Spring - western side & 2.29 & 2.15 & 2.40 & 2.12 & 2.15 \\
\hline 08WA121, Unnamed pool near The Gap & 3.40 & 3.24 & 3.28 & 2.58 & 3.24 \\
\hline $\begin{array}{l}\text { 08WA122, Unnamed pool upslope from } \\
\text { "Bullseye Spring," Geyser Springs Group }\end{array}$ & 2.40 & 2.27 & 2.49 & 2.23 & 2.27 \\
\hline $\begin{array}{l}08 \mathrm{WA} 12340 \mathrm{~cm} \text { diameter circular clay } \\
\text { hot spring } 2 \text { meters from } 08 \mathrm{WA} 121\end{array}$ & 2.80 & 2.79 & 2.87 & 2.70 & 2.80 \\
\hline $\begin{array}{l}\text { 08WA124, Appendage side spring to } \\
\text { "Lifeboat Spring" }\end{array}$ & 2.64 & 2.52 & 2.74 & 2.38 & 2.52 \\
\hline 08WA125 "Lifeboat Spring" & 3.23 & 3.23 & 3.35 & 2.57 & 3.23 \\
\hline 08WA126, Echinus Geyser & 3.52 & 3.37 & 3.44 & 3.03 & 3.52 \\
\hline 08WA131, "Kaolin Spring" & 2.54 & 2.51 & 2.67 & 2.54 & 2.54 \\
\hline
\end{tabular}




\section{Water-Chemistry Data}

Site data and water analyses for YNP thermal features, their overflow drainage channels, and surface waters sampled in 2006 to 2008 are presented in tables 1 and 5-13. To organize presentation of the results, samples are sorted first by basin, then alphabetically by spring, then by date of sample collection, and then by sampling site along the drainage channel, if present. Photographs of most of the sample sites are in the Appendix.

\section{Overflow Drainage Chemical Profiles}

Samples were collected from four overflow drainage channels to investigate changes in $\mathrm{S}, \mathrm{Fe}, \mathrm{As}, \mathrm{Sb}$, and $\mathrm{N}$ redox species and loss of solutes through volatilization or precipitation. Results are plotted on the same distance scales for each of the respective transects for comparison. For the 2006 Succession Spring transect sampling event, sample temperature, $\mathrm{pH}$, specific conductance, and concentrations of $\mathrm{Ca}, \mathrm{SO}_{4}, \mathrm{Cl}, \mathrm{F}$, and $\mathrm{Al}$ are plotted as a function of distance from the discharge source (figs. $22 \mathrm{~A}, \mathrm{~B}, \mathrm{C}$, and D). Concentrations of $\mathrm{Hg}$, As(total), $\mathrm{Fe}\left(\right.$ total), $\mathrm{Fe}(\mathrm{II}), \mathrm{Fe}(\mathrm{III}), \mathrm{Na}, \mathrm{NH}_{4}$, and delta-deuterium and delta-oxygen-18 are plotted on figures $23 \mathrm{~A}, \mathrm{~B}, \mathrm{C}$, and D. Specific conductance, concentrations of $\mathrm{SO}_{4}, \mathrm{Cl}, \mathrm{Al}$, and $\mathrm{Fe}(\mathrm{III})$, and $\delta \mathrm{D}$ and $\delta^{18} \mathrm{O}$ increased with distance, whereas temperature, $\mathrm{pH}$, and concentrations of $\mathrm{Hg}$, As(total), $\mathrm{NH}_{4}$, and $\mathrm{Fe}$ (II) decreased with distance, and concentrations of $\mathrm{Ca}, \mathrm{F}, \mathrm{Na}$, and $\mathrm{Fe}$ (total) were essentially unchanged.

For the 2008 Ojo Caliente transect sampling event, sample temperature, $\mathrm{pH}$, field and laboratory specific conductance, and concentrations of $\mathrm{SO}_{4}, \mathrm{Cl}, \mathrm{Li}$, and $\mathrm{B}$ are plotted as a function of distance from the discharge source on figures $24 \mathrm{~A}, \mathrm{~B}, \mathrm{C}$, and $\mathrm{D}$. Concentrations of $\mathrm{Na}, \mathrm{K}, \mathrm{NO}_{3}$, dissolved oxygen, and As(total), and delta-deuterium and delta-oxygen-18 are plotted as a function of distance from the discharge source on figures $25 \mathrm{~A}, \mathrm{~B}$, and $\mathrm{C}$. Sample $\mathrm{pH}$, field and laboratory specific conductance, and concentrations of $\mathrm{SO}_{4}, \mathrm{Cl}, \mathrm{Li}$, and $\mathrm{B}, \mathrm{Na}, \mathrm{K}, \mathrm{NO}_{3}$, dissolved oxygen and As(total), and delta-deuterium and delta-oxygen-18 increased with distance, whereas temperature decreased with distance and $\mathrm{K}$ concentration was essentially unchanged.

For the upper Rabbit Creek area transect sampling event (06WA114), sample temperature, $\mathrm{pH}$, specific conductance, concentrations of $\mathrm{Ca}, \mathrm{SO}_{4}, \mathrm{Cl}, \mathrm{F}, \mathrm{Al}$, are plotted as a function of distance from the discharge source on figures $26 \mathrm{~A}, \mathrm{~B}, \mathrm{C}$, and D. Concentrations of $\mathrm{Na}, \mathrm{Li}, \mathrm{B}, \mathrm{Sb}$ (total), $\mathrm{Hg}$, and $\mathrm{As}$ (total), and delta-deuterium and delta-oxygen-18 are plotted on figures $27 \mathrm{~A}, \mathrm{~B}, \mathrm{C}$, and D. Sample $\mathrm{pH}$, specific conductance, concentrations of $\mathrm{Ca}, \mathrm{SO}_{4}, \mathrm{Cl}, \mathrm{F}$, $\mathrm{Al}, \mathrm{Na}, \mathrm{Li}, \mathrm{B}$, and As(total), and delta-deuterium and delta-oxygen-18 increased with distance, whereas temperature and concentrations of $\mathrm{Sb}$ (total) and $\mathrm{Hg}$ decreased with distance.

For the lower Rabbit Creek area transect sampling event (06WA116), sample temperature, $\mathrm{pH}$, specific conductance, concentrations of $\mathrm{Ca}, \mathrm{SO}_{4}, \mathrm{Cl}, \mathrm{F}, \mathrm{Al}$, are plotted as a function of distance from the discharge source on figures $28 \mathrm{~A}, \mathrm{~B}, \mathrm{C}$, and D. Concentrations of $\mathrm{B}, \mathrm{Li}, \mathrm{Na}, \mathrm{HCO}_{3}, \mathrm{Hg}$, and $\mathrm{As}$ (total), and delta-deuterium and delta-oxygen-18 are plotted on figures $29 \mathrm{~A}, \mathrm{~B}, \mathrm{C}$, and D. Sample $\mathrm{pH}$, specific conductance, concentrations of $\mathrm{Ca}, \mathrm{SO}_{4}, \mathrm{Cl}, \mathrm{F}$, $\mathrm{Al}, \mathrm{Na}, \mathrm{B}, \mathrm{Na}$, alkalinity, and As(total), and delta-deuterium and delta-oxygen-18 increased with distance, whereas temperature and concentration of $\mathrm{Hg}$ decreased with distance.

Mercury results, plotted on figures 23 A, 27 C, and 29 C, illustrate generally decreasing $\mathrm{Hg}(\mathrm{T})$ concentrations with distance. Mercury concentration in the Succession Spring transect decreased from 127 to $21 \mathrm{ng} / \mathrm{L}$, and mercury concentration in the lower Rabbit Creek transect decreased from 175 to $70 \mathrm{ng} / \mathrm{L}$. 

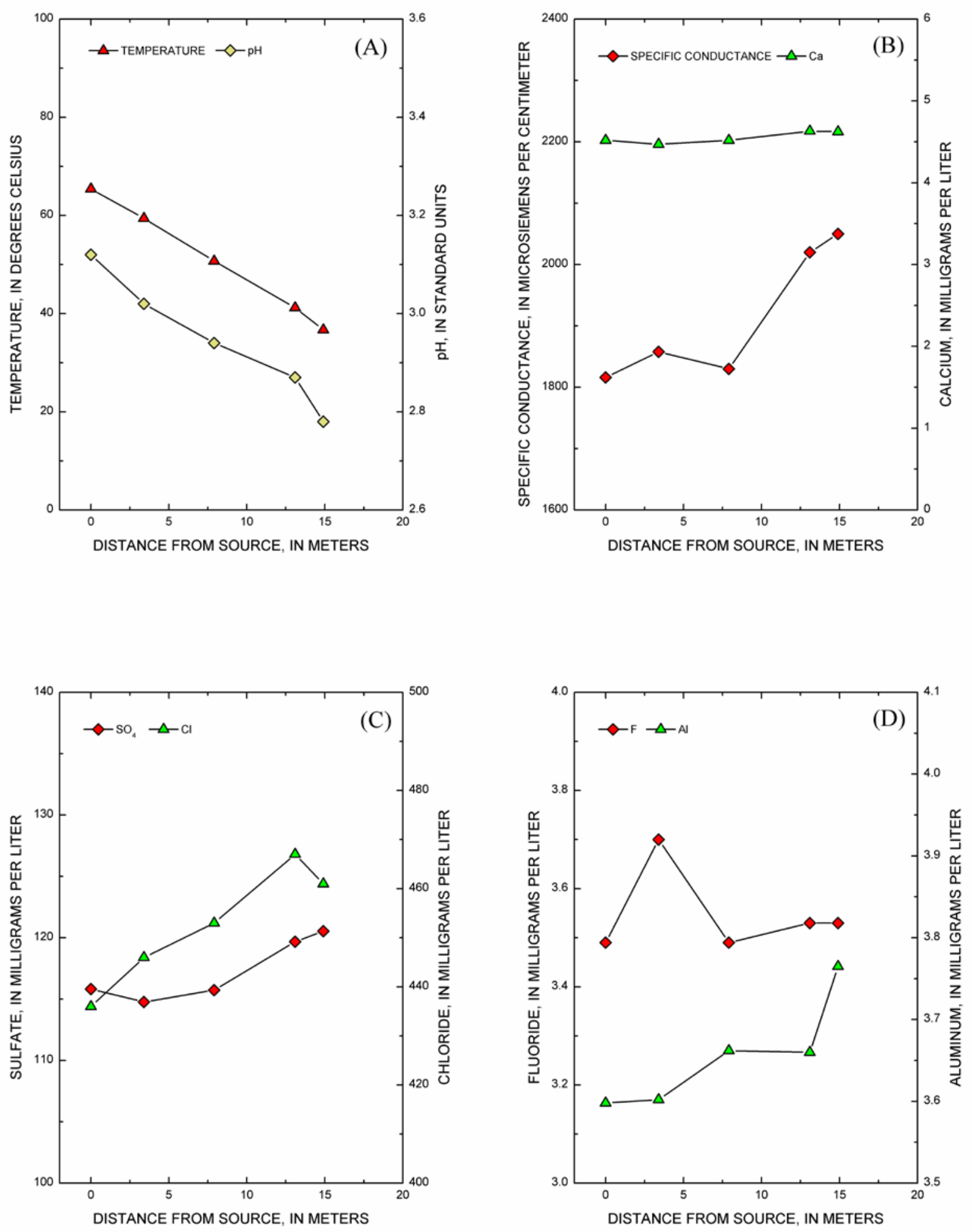

Figure 22. Sample temperature and pH (A), specific conductance and concentration of calcium (B), concentrations of sulfate and chloride (C), and concentrations of fluoride and aluminum (D) as a function of drainage distance for Succession Spring and its overflow drainage channel for samples collected in 2006. 

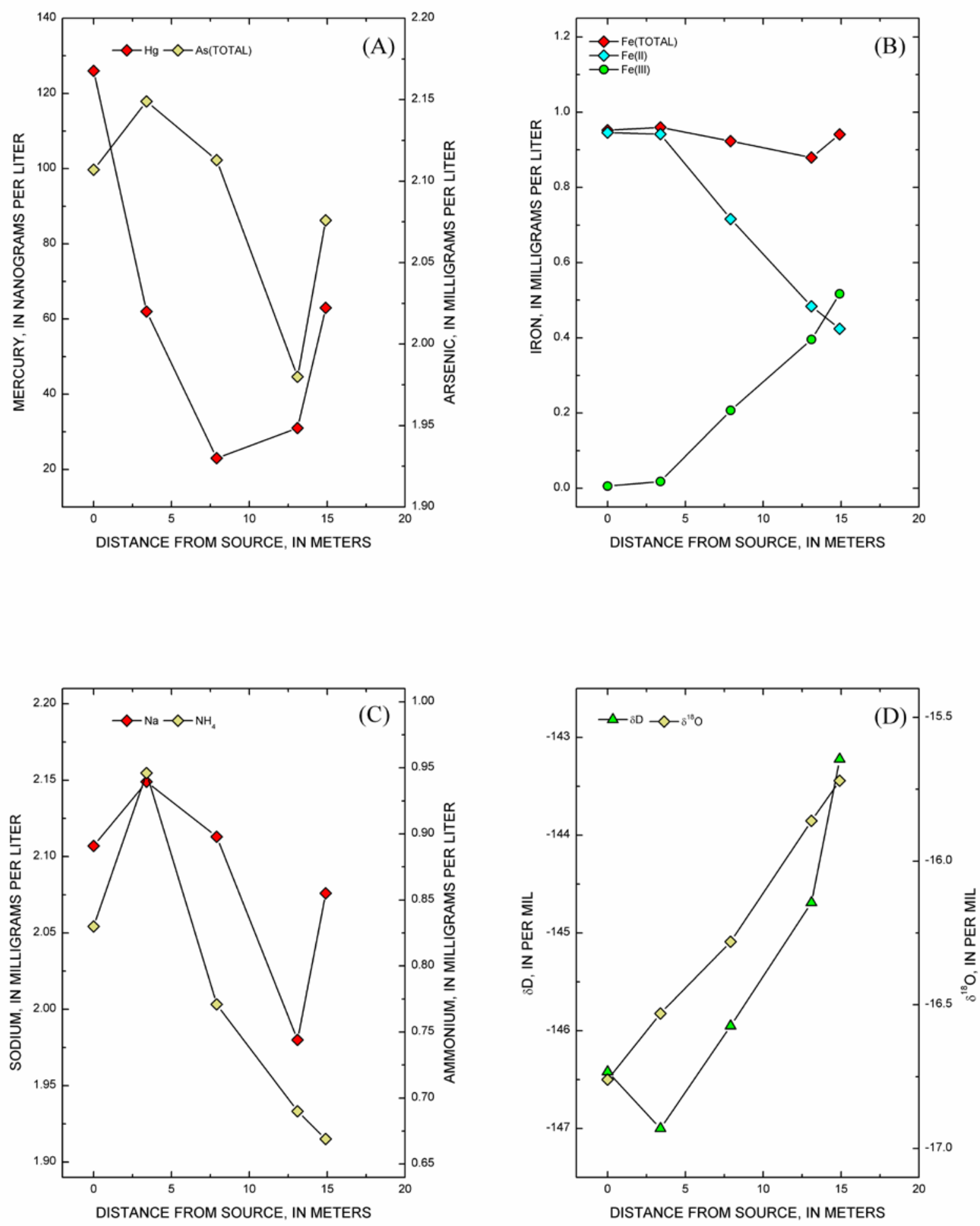

Figure 23. Concentrations of mercury and arsenic(total) (A), concentrations of iron (total), iron (II), and iron (III) (B), concentrations of sodium and ammonium (C), and delta deuterium and delta ${ }^{18} \mathrm{O}$ (D) as a function of drainage distance for Succession Spring and its overflow drainage channel for samples collected in 2006. 

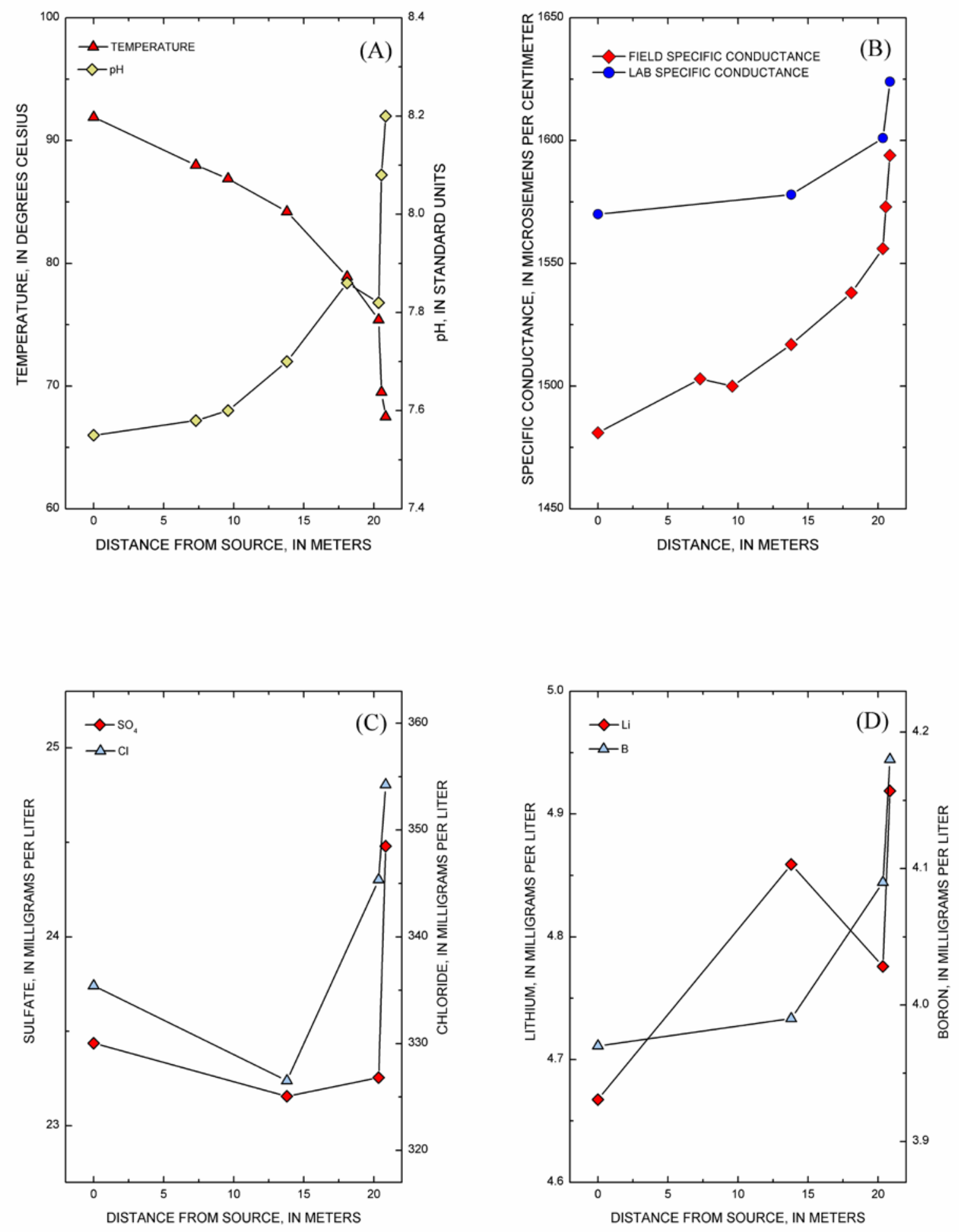

Figure 24. Sample temperature and $\mathrm{pH}(\mathrm{A})$, field and laboratory specific conductance (B), concentrations of sulfate and chloride (C), and concentrations of lithium and boron (D) as a function of drainage distance for Ojo Caliente Spring and its overflow drainage channel for samples collected in 2008. 

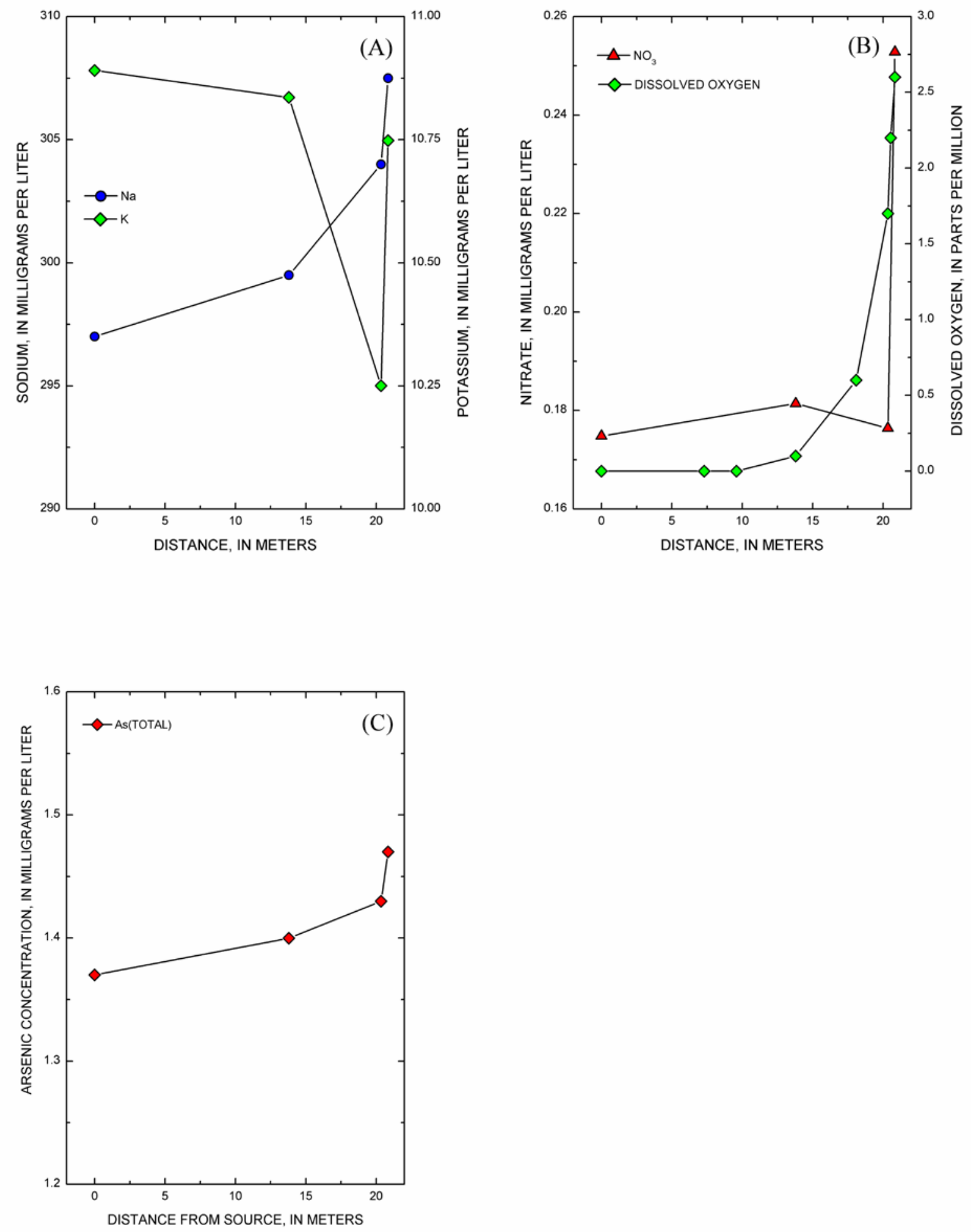

Figure 25. Concentrations of sodium and potassium (A), concentrations of nitrate and dissolved oxygen (B) and concentration of arsenic (total) (C) as a function of drainage distance for Ojo Caliente Spring and its overflow drainage channel for samples collected in 2008. 

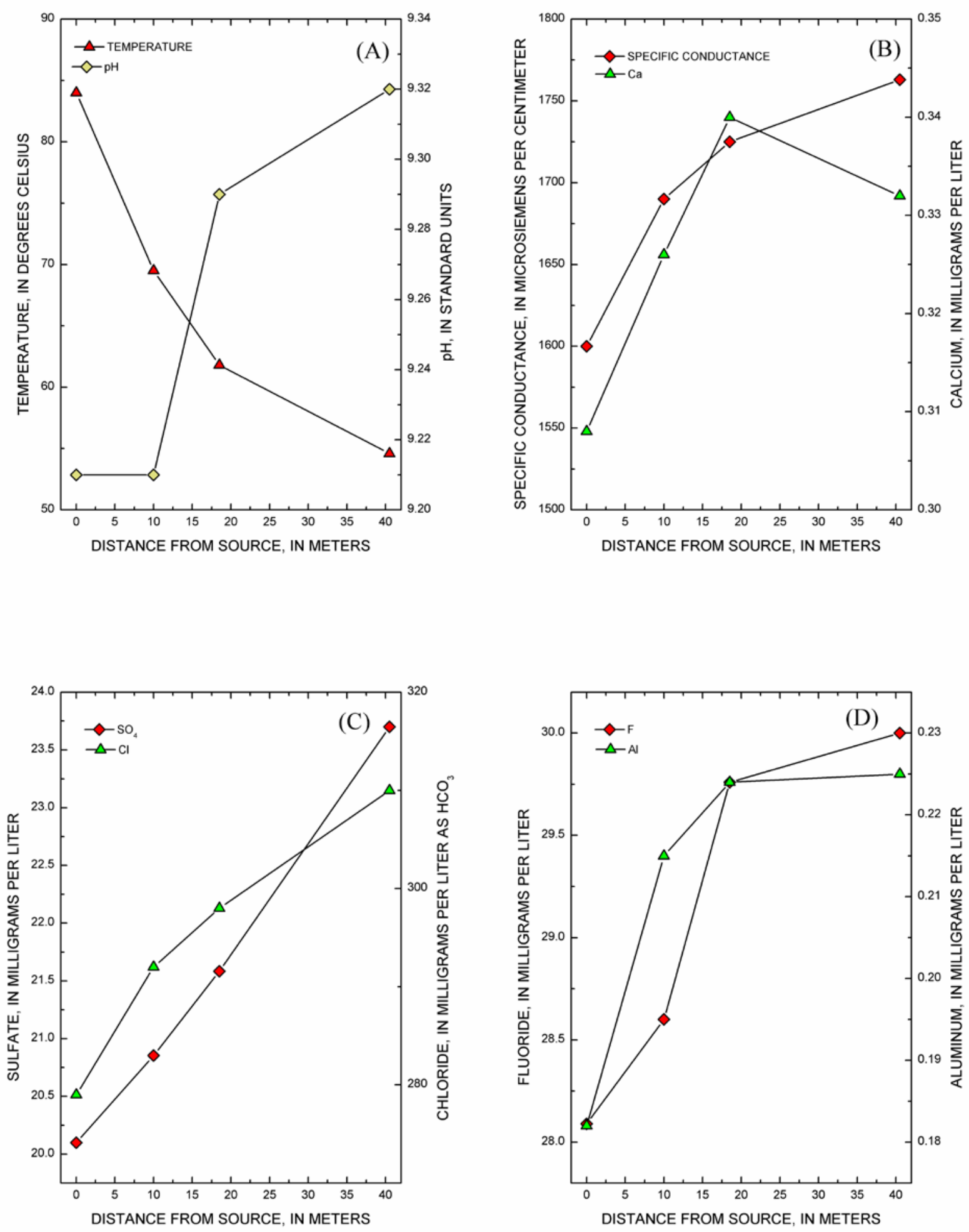

Figure 26. Sample temperature and pH (A), specific conductance and concentration of calcium (B), concentrations of sulfate and chloride (C) and concentrations of fluoride and aluminum (D) as a function of drainage distance for an unnamed thermal feature and its overflow drainage channel in the upper Rabbit Creek area for samples collected in 2006. 

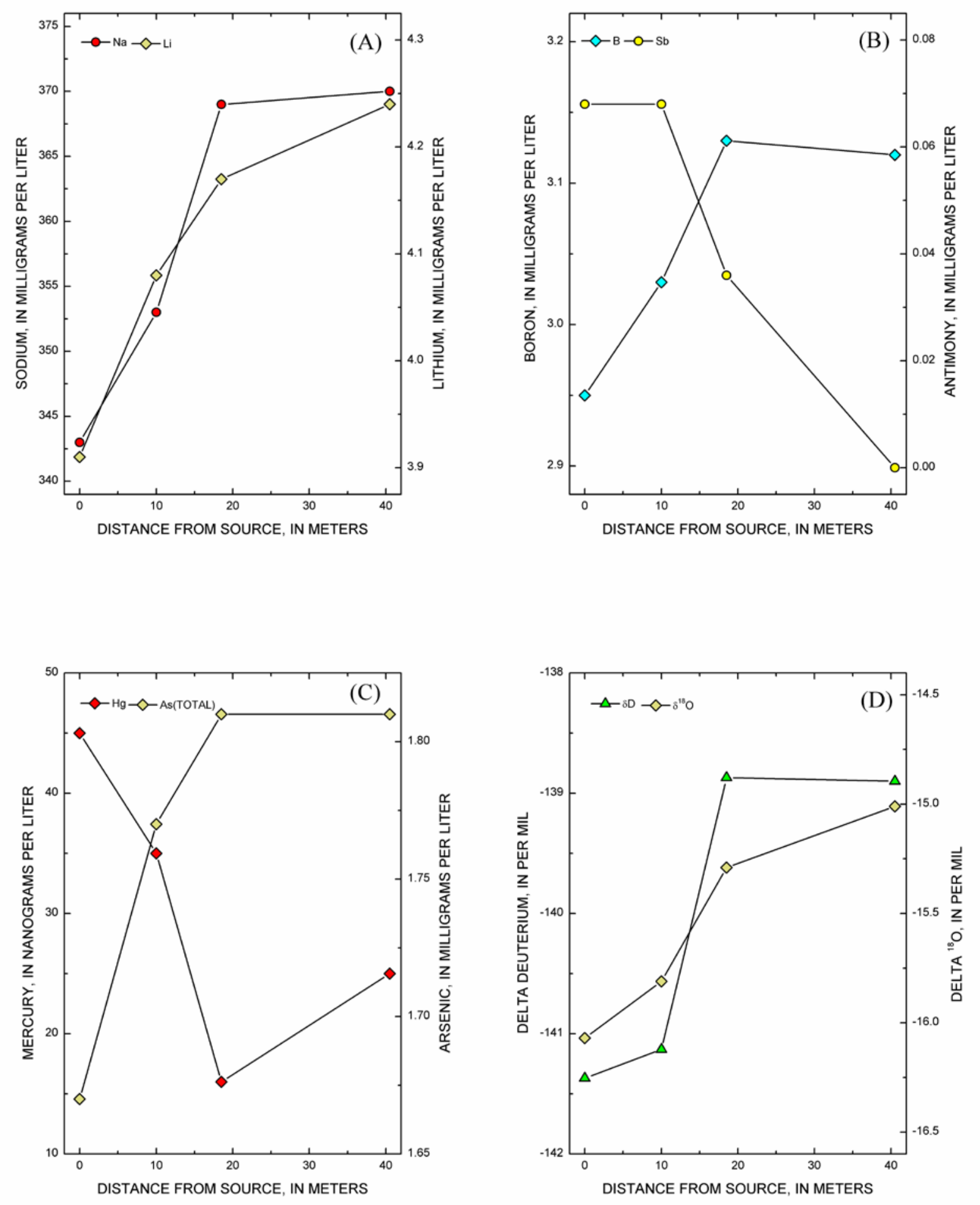

Figure 27. Concentrations of sodium and lithium (A), concentrations of boron and antimony (total) (B), concentrations of mercury and arsenic (total) (C), and delta deuterium and delta ${ }^{18} \mathrm{O}(\mathrm{D})$ as a function of drainage distance for an unnamed thermal feature and its overflow drainage channel in the upper Rabbit Creek area for samples collected in 2006. 

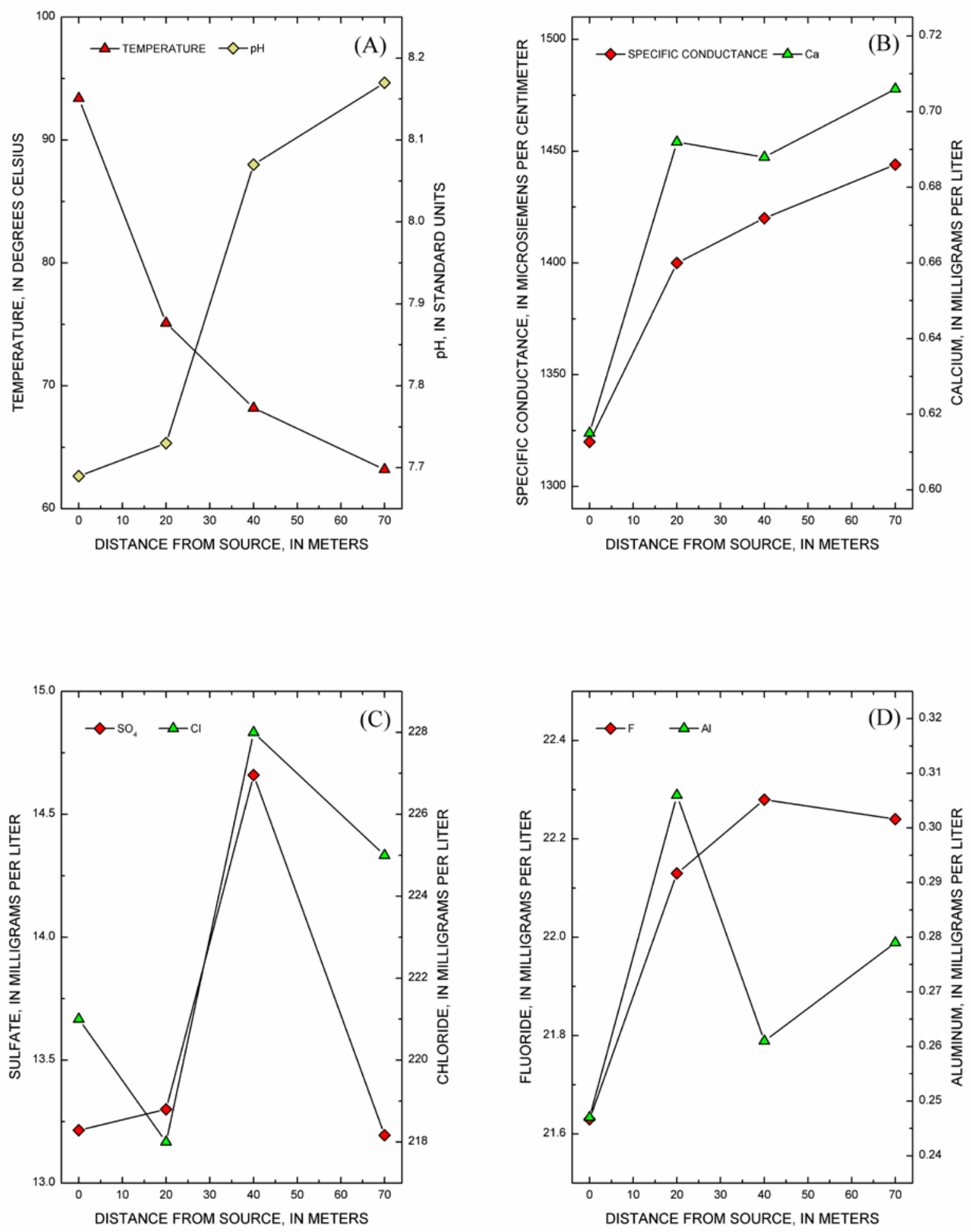

Figure 28. Sample temperature and $\mathrm{pH}(\mathrm{A})$, specific conductance, and concentration of calcium (B), concentrations of sulfate and chloride (C), and concentrations of fluoride and aluminum (D) as a function of drainage distance for an unnamed thermal feature and its overflow drainage channel in the lower Rabbit Creek area for samples collected in 2006. 

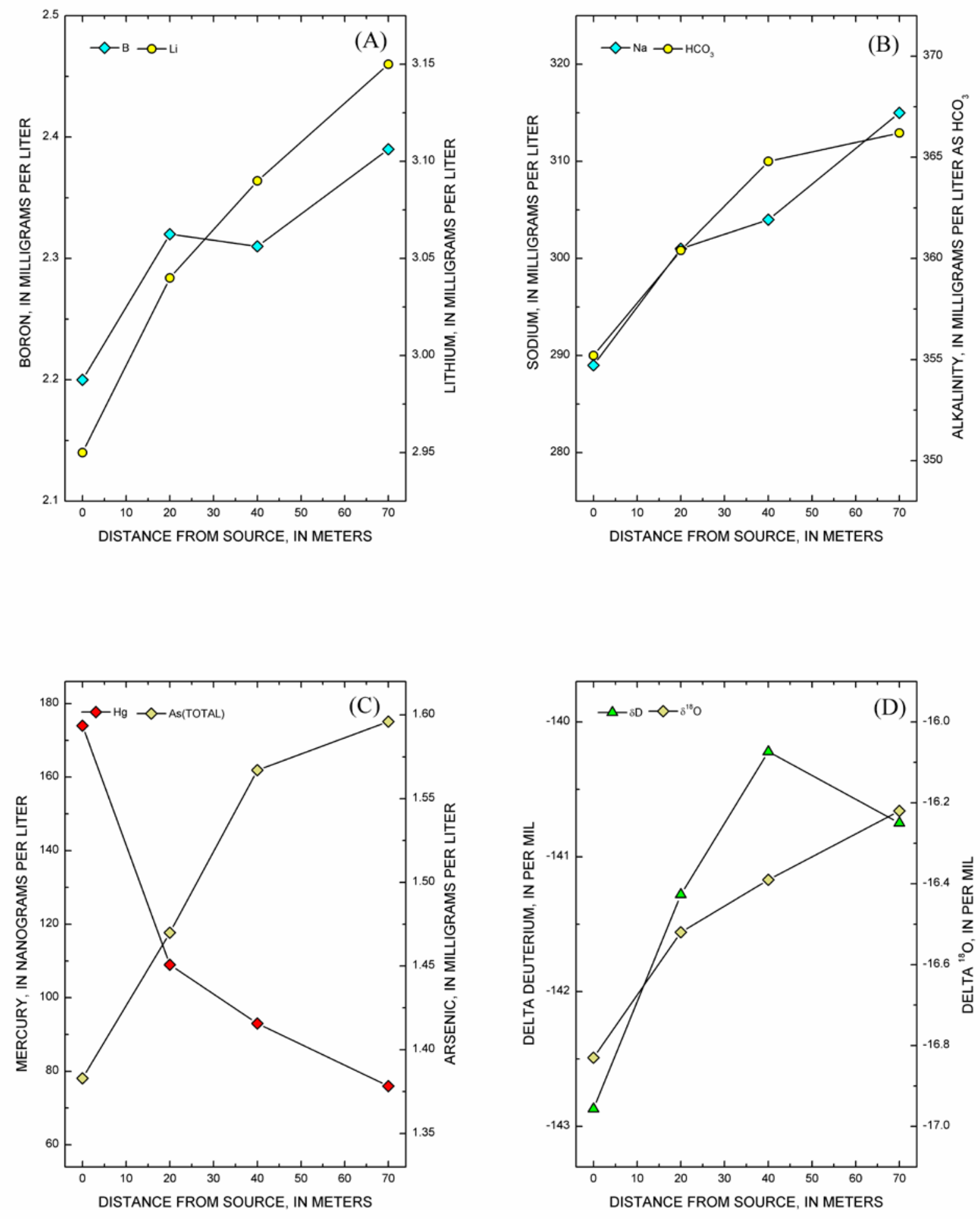

Figure 29. Concentrations of boron and lithium (A), concentrations of sodium and bicarbonate (B), concentrations of mercury and arsenic(total) (C), and delta deuterium and delta 180 (D) as a function of drainage distance for an unnamed thermal feature and its overflow drainage channel in the lower Rabbit Creek area for samples collected in 2006. 
Table 5. Results of water analyses for Norris-Mammoth corridor and the West Nymph Creek thermal area, 2006-2008.

[ $\delta \mathrm{D}$ and $\delta 18 \mathrm{O}$, isotopic composition of hydrogen and oxygen relative to VSMOW; $\mathrm{m}$, meters; meq/L, milliequivalents per liter; $\mathrm{mg} / \mathrm{L}$, milligrams per liter; $\mathrm{mM}$, millimolar; $\mu \mathrm{S} / \mathrm{cm}$, microsiemens per centimeter; ng/L nanograms per liter; $\mathrm{V}$, volts; ---, not measured; ${ }^{\circ} \mathrm{C}$, degrees Celsius; $<$, less than]

\begin{tabular}{|c|c|c|c|c|}
\hline & \multicolumn{2}{|c|}{ Norris-Mammoth Corridor } & \multicolumn{2}{|c|}{ West Nymph Creek Thermal Area } \\
\hline Location & Apollinaris Spring & Nymphy Creek & $\begin{array}{l}\text { Unnamed most upslope } \\
\text { pool }\end{array}$ & $\begin{array}{l}\text { Unnamed sunken } \\
\text { circular pool }\end{array}$ \\
\hline Sample ID & 06WA124 & 06WA136 & 08WA118 & 08WA119 \\
\hline Collection Date / Time & $5 / 16 / 0612: 20$ & $9 / 12 / 0610: 00$ & 9/22/08 16:00 & $9 / 22 / 0817: 20$ \\
\hline Temperature, ${ }^{\circ} \mathrm{C}$ & 10.5 & 15.5 & 61.8 & 58.1 \\
\hline pH (field / laboratory) & $5.16 / 8.08$ & $6.86 / 7.64$ & $5.71 / 8.09$ & $1.60 / 1.60$ \\
\hline Specific conductance & & & & \\
\hline (field / laboratory), $\mu \mathrm{S} / \mathrm{cm}$ & $138 / 136$ & $741 / 739$ & $347 / 350$ & $7650 / 11,830$ \\
\hline $\mathrm{Eh}, \mathrm{V}$ & 0.448 & 0.445 & 0.120 & 0.570 \\
\hline Density, $\mathrm{g} / \mathrm{mL}$ at $20^{\circ} \mathrm{C}$ & 0.9983 & 0.9987 & 0.9985 & 1.0004 \\
\hline Dissolved oxygen (DO), mg/L & --- & 7.0 & --- & --- \\
\hline \multicolumn{5}{|l|}{ Constituent, $\mathrm{mg} / \mathrm{L}^{1}$} \\
\hline Calcium (Ca) & 13.8 & 11.4 & 6.05 & 4.32 \\
\hline Magnesium (Mg) & 3.46 & 1.25 & 0.376 & 0.880 \\
\hline Sodium (Na) & 6.94 & 137 & 58.6 & 25.5 \\
\hline Potassium (K) & 3.06 & 13.2 & 4.67 & 32.2 \\
\hline Strontium (Sr) & 0.047 & 0.027 & 0.011 & 0.018 \\
\hline Barium (Ba) & 0.004 & 0.014 & 0.014 & 0.065 \\
\hline Lithium (Li) & 0.021 & 0.649 & 0.303 & 0.048 \\
\hline Sulfate $\left(\mathrm{SO}_{4}\right)$ & 5.15 & 64.3 & 46.0 & 2820 \\
\hline Thiosulfate $\left(\mathrm{S}_{2} \mathrm{O}_{3}\right)$ & --- & --- & --- & $<0.1$ \\
\hline Polythionate $\left(\mathrm{S}_{\mathrm{n}} \mathrm{O}_{6}\right), \mathrm{mM}$ & --- & -- & --- & $<0.001$ \\
\hline Hydrogen sulfide $\left(\mathrm{H}_{2} \mathrm{~S}\right)$ & --- & --- & --- & --- \\
\hline Alkalinity $\left(\mathrm{HCO}_{3}\right)$ & 71.3 & 198 & 95.9 & --- \\
\hline Acidity (free / total), mM & --- & --- & --- & $34.2 / 42.4$ \\
\hline Fluoride (F) & 4.39 & 5.18 & 6.28 & 0.370 \\
\hline Chloride (Cl) & 0.667 & 76.8 & 7.68 & 4.54 \\
\hline Bromide (Br) & $<0.03$ & 0.23 & $<0.03$ & $<0.03$ \\
\hline Nitrate $\left(\mathrm{NO}_{3}\right)$ & 0.37 & 0.12 & 0.09 & 0.19 \\
\hline Ammonium $\left(\mathrm{NH}_{4}\right)$ & $<0.07$ & $<0.07$ & 0.300 & 29.0 \\
\hline Silica $\left(\mathrm{SiO}_{2}\right)$ & 54.5 & 115 & 96.8 & 202 \\
\hline Boron (B) & 0.013 & 1.15 & 0.227 & 0.556 \\
\hline Aluminum (Al) & 1.95 & 0.20 & 0.24 & 59.3 \\
\hline Iron total $(\mathrm{Fe}(\mathrm{T}))$ & 0.005 & 0.050 & 0.014 & 17.7 \\
\hline Ferrous iron (Fe(II)) & $<0.002$ & 0.050 & 0.014 & 17.0 \\
\hline Manganese (Mn) & 0.011 & 0.012 & 0.139 & 0.523 \\
\hline Copper $(\mathrm{Cu})$ & 0.0042 & $<0.0005$ & $<0.0005$ & $<0.0005$ \\
\hline Zinc (Zn) & 0.022 & $<0.004$ & $<0.004$ & 0.129 \\
\hline Cadmium (Cd) & 0.0002 & $<0.0001$ & $<0.0001$ & 0.0002 \\
\hline Chromium (Cr) & 0.0006 & $<0.0005$ & $<0.0005$ & 0.0042 \\
\hline Cobalt (Co) & $<0.0007$ & $<0.0007$ & $<0.0007$ & $<0.0007$ \\
\hline Mercury total (Hg), ng/L & 11.0 & 11.0 & 94.4 & 140 \\
\hline Methylmercury $\left(\mathrm{CH}_{3} \mathrm{Hg}\right), \mathrm{ng} / \mathrm{L}$ & --- & 0.21 & 0.15 & 0.05 \\
\hline Nickel (Ni) & $<0.0005$ & $<0.0005$ & $<0.0005$ & $<0.0005$ \\
\hline Lead $(\mathrm{Pb})$ & $<0.0008$ & $<0.0008$ & $<0.0008$ & 0.040 \\
\hline Beryllium (Be) & 0.004 & $<0.001$ & 0.001 & 0.005 \\
\hline Vanadium (V) & $<0.005$ & $<0.005$ & $<0.005$ & 0.008 \\
\hline Molybdenum (Mo) & $<0.007$ & $<0.007$ & 0.010 & $<0.007$ \\
\hline Antimony (Sb) & $<0.001$ & 0.006 & $<0.001$ & $<0.001$ \\
\hline Selenium (Se) & $<0.001$ & $<0.001$ & $<0.001$ & $<0.001$ \\
\hline Arsenic (As) & 0.0015 & 0.307 & 0.001 & 0.002 \\
\hline Arsenite (As(III)) & 0.0011 & 0.006 & 0.001 & 0.002 \\
\hline Dissolved organic carbon (DOC) & 1.7 & 2.7 & 1.1 & 10.5 \\
\hline$\delta \mathrm{D}$, per mil & -139.03 & -134.74 & -139.64 & -103.66 \\
\hline$\delta^{18} 0$, per mil & -18.39 & -16.46 & -17.13 & -4.06 \\
\hline sum cations, meq/L & 1.4 & 7.0 & 3.0 & 36.7 \\
\hline sum anions, meq/L & 1.4 & 7.0 & 3.0 & 34.5 \\
\hline Charge imbalance, percent & 4.7 & 1.0 & 0.8 & 6.1 \\
\hline
\end{tabular}

${ }^{1}$ Except for acidity (mM), mercury (ng/L), methylmercury (ng/L), and $\delta \mathrm{D}$ and $\delta^{18} \mathrm{O}$ (per mil) 
Table 6. Results of water analyses for Norris Geyser Basin, 2006-2008.

$[\delta \mathrm{D}$ and $\delta 18 \mathrm{O}$, isotopic composition of hydrogen and oxygen relative to VSMOW; m, meters; meq/L, milliequivalents per liter; $\mathrm{mg} / \mathrm{L}$, milligrams per liter; $\mathrm{mM}$, millimolar; $\mu \mathrm{S} / \mathrm{cm}$, microsiemens per centimeter; $\mathrm{ng} / \mathrm{L}$ nanograms per liter; $\mathrm{V}$, volts; ---, not measured; ${ }^{\circ} \mathrm{C}$, degrees Celsius; $<$, less than]

\begin{tabular}{|c|c|c|c|c|}
\hline \multirow[b]{2}{*}{ Location } & \multicolumn{4}{|c|}{ 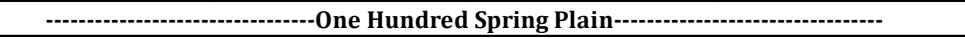 } \\
\hline & Cinder Pool & $\begin{array}{c}\text { Realgar Creek near } \\
\text { mouth }\end{array}$ & $\begin{array}{c}\text { Realgar Creek near } \\
\text { mouth }\end{array}$ & Tantalus Creek at weir \\
\hline Sample ID & 07WA113 & 06WA106 & 06WA139 & 06WA107 \\
\hline Collection Date / Time & $9 / 12 / 0713: 00$ & $5 / 13 / 0611: 30$ & 9/12/06 15:00 & $5 / 13 / 0613: 15$ \\
\hline Temperature, ${ }^{\circ} \mathrm{C}$ & 86.8 & 31.2 & 28.2 & 31.2 \\
\hline pH (field / laboratory) & $4.28 / 3.96$ & $2.99 / 3.00$ & $3.05 / 3.00$ & $3.09 / 3.11$ \\
\hline \multicolumn{5}{|l|}{ Specific conductance } \\
\hline (field / laboratory), $\mu \mathrm{S} / \mathrm{cm}$ & $2090 / 2260$ & $2210 / 2260$ & $2330 / 2340$ & 1998 / 2040 \\
\hline Eh, V & -0.042 & 0.329 & 0.702 & 0.381 \\
\hline Density, $\mathrm{g} / \mathrm{mL}$ at $20^{\circ} \mathrm{C}$ & 0.9994 & 0.9992 & 0.9993 & 0.9991 \\
\hline Dissolved oxygen (DO), mg/L & $--\cdot$ & --- & $--\cdot$ & 7.5 \\
\hline \multicolumn{5}{|l|}{ Constituent, $\mathrm{mg} / \mathrm{L}^{1}$} \\
\hline Calcium (Ca) & 5.22 & 4.49 & 4.58 & 3.59 \\
\hline Magnesium (Mg) & 0.020 & 0.226 & 0.210 & 0.242 \\
\hline Sodium (Na) & 409 & 292 & 310 & 263 \\
\hline Potassium (K) & 41.4 & 44.9 & 50.1 & 44.7 \\
\hline Strontium (Sr) & 0.015 & 0.028 & 0.025 & 0.014 \\
\hline Barium (Ba) & 0.015 & 0.101 & 0.111 & 0.076 \\
\hline Lithium (Li) & 3.96 & 2.28 & 2.72 & 2.87 \\
\hline Sulfate $\left(\mathrm{SO}_{4}\right)$ & 63.7 & 152 & 152 & 133 \\
\hline Thiosulfate $\left(\mathrm{S}_{2} \mathrm{O}_{3}\right)$ & 19.4 & --- & --- & --- \\
\hline Polythionate $\left(\mathrm{S}_{\mathrm{n}} \mathrm{O}_{6}\right), \mathrm{mM}$ & $<0.001$ & -- & -- & -- \\
\hline Hydrogen sulfide $\left(\mathrm{H}_{2} \mathrm{~S}\right)$ & 2.12 & --- & --- & --- \\
\hline Alkalinity $\left(\mathrm{HCO}_{3}\right)$ & --- & --- & --- & --- \\
\hline Acidity (free / total), mM & --- & $1.15 / 1.90$ & $1.02 / 1.71$ & $0.93 / 1.40$ \\
\hline Fluoride (F) & 6.83 & 4.12 & 4.31 & 3.75 \\
\hline Chloride $(\mathrm{Cl})$ & 633 & 456 & 499 & 426 \\
\hline Bromide (Br) & 1.82 & 1.51 & 1.63 & 1.42 \\
\hline Nitrate $\left(\mathrm{NO}_{3}\right)$ & $<0.05$ & 0.11 & $<0.05$ & 0.09 \\
\hline Ammonium $\left(\mathrm{NH}_{4}\right)$ & 6.77 & 2.18 & 1.29 & 1.97 \\
\hline Silica $\left(\mathrm{SiO}_{2}\right)$ & 383 & 250 & 261 & 315 \\
\hline Boron (B) & 9.70 & 6.96 & 8.06 & 6.17 \\
\hline Aluminum (Al) & 0.95 & 3.99 & 3.59 & 2.47 \\
\hline Iron total $(\mathrm{Fe}(\mathrm{T}))$ & 0.012 & 1.20 & 1.10 & 1.07 \\
\hline Ferrous iron (Fe(II)) & 0.012 & 0.527 & 0.296 & 0.437 \\
\hline Manganese (Mn) & 0.004 & 0.038 & 0.037 & 0.066 \\
\hline Copper $(\mathrm{Cu})$ & $<0.0005$ & $<0.0005$ & 0.0006 & $<0.0005$ \\
\hline Zinc (Zn) & $<0.004$ & 0.008 & 0.009 & 0.008 \\
\hline Cadmium (Cd) & $<0.0001$ & 0.0003 & $<0.0001$ & $<0.0001$ \\
\hline Chromium (Cr) & $<0.0005$ & 0.0006 & $<0.0005$ & 0.0009 \\
\hline Cobalt (Co) & $<0.0007$ & $<0.0007$ & $<0.0007$ & 0.0007 \\
\hline Mercury total (Hg), ng/L & 53.0 & --- & 22.0 & --- \\
\hline Methylmercury $\left(\mathrm{CH}_{3} \mathrm{Hg}\right), \mathrm{ng} / \mathrm{L}$ & 0.04 & --- & 0.05 & --- \\
\hline Nickel (Ni) & $<0.0005$ & $<0.0005$ & $<0.0005$ & $<0.0005$ \\
\hline Lead $(\mathrm{Pb})$ & $<0.0008$ & $<0.0008$ & $<0.0008$ & 0.0008 \\
\hline Beryllium (Be) & $<0.001$ & 0.002 & 0.002 & 0.002 \\
\hline Vanadium (V) & $<0.005$ & $<0.005$ & $<0.005$ & $<0.005$ \\
\hline Molybdenum (Mo) & 0.108 & $<0.007$ & $<0.007$ & 0.048 \\
\hline Antimony (Sb) & 0.019 & 0.033 & 0.041 & 0.050 \\
\hline Selenium (Se) & $<0.001$ & $<0.001$ & $<0.001$ & $<0.001$ \\
\hline Arsenic (As) & 2.35 & 1.37 & 1.52 & 1.48 \\
\hline Arsenite (As(III)) & $1.09^{2}$ & 0.061 & 0.035 & 0.032 \\
\hline Dissolved organic carbon (DOC) & 0.8 & 0.9 & 0.8 & 1.4 \\
\hline$\delta \mathrm{D}$, per mil & -125.31 & -135.89 & -131.64 & -132.16 \\
\hline$\delta^{18} 0$, per mil & -10.69 & -13.94 & -13.27 & -13.61 \\
\hline sum cations, meq/L & 20.1 & 15.9 & 16.6 & 14.3 \\
\hline sum anions, meq/L & 19.4 & 15.9 & 17.1 & 14.7 \\
\hline Charge imbalance, percent & 3.7 & 0.2 & -3.0 & -2.7 \\
\hline
\end{tabular}


Table 6. Results of water analyses for Norris Geyser Basin, 2006-2008-Continued.

\begin{tabular}{|c|c|c|c|}
\hline & \multicolumn{3}{|c|}{ - } \\
\hline Location & Tantalus Creek at weir & Tantalus Creek at weir & $\begin{array}{l}\text { Unnamed thermal } \\
\text { feature northeast of } \\
\text { Cinder Pool }\end{array}$ \\
\hline Sample ID & 06WA158 & 08WA105 & 06WA109 \\
\hline Collection Date / Time & 9/14/06 18:30 & $9 / 17 / 0815: 30$ & 5/13/06 15:50 \\
\hline Temperature, ${ }^{\circ} \mathrm{C}$ & 23.6 & 31.5 & 74.0 \\
\hline pH (field / laboratory) & $3.02 / 3.02$ & $3.06 / 3.06$ & $3.56 / 3.45$ \\
\hline $\begin{array}{l}\text { Specific conductance } \\
\text { (field / laboratory), } \mu \mathrm{S} / \mathrm{cm}\end{array}$ & $2290 / 2300$ & $2140 / 2150$ & 1720 / 1880 \\
\hline Eh, V & --- & 0.670 & 0.142 \\
\hline Density, $\mathrm{g} / \mathrm{mL}$ at $20^{\circ} \mathrm{C}$ & 0.9993 & 0.9991 & 0.9991 \\
\hline Dissolved oxygen (DO), mg/L & --- & --- & --- \\
\hline \multicolumn{4}{|l|}{ Constituent, mg/L ${ }^{1}$} \\
\hline Calcium (Ca) & 4.09 & 3.49 & 3.41 \\
\hline Magnesium (Mg) & 0.231 & 0.204 & 0.223 \\
\hline Sodium (Na) & 310 & 330 & 269 \\
\hline Potassium (K) & 56.3 & 53.5 & 46.8 \\
\hline Strontium (Sr) & 0.016 & 0.014 & 0.019 \\
\hline Barium (Ba) & 0.083 & 0.079 & 0.164 \\
\hline Lithium (Li) & 3.93 & 3.54 & 2.92 \\
\hline Sulfate $\left(\mathrm{SO}_{4}\right)$ & 155 & 152 & 91.2 \\
\hline Thiosulfate $\left(\mathrm{S}_{2} \mathrm{O}_{3}\right)$ & --- & --- & $<0.1$ \\
\hline Polythionate $\left(\mathrm{S}_{\mathrm{n}} \mathrm{O}_{6}\right), \mathrm{mM}$ & -- & --- & $<0.001$ \\
\hline Hydrogen sulfide $\left(\mathrm{H}_{2} \mathrm{~S}\right)$ & --- & --- & 0.30 \\
\hline Alkalinity $\left(\mathrm{HCO}_{3}\right)$ & --- & --- & --- \\
\hline Acidity (free / total), mM & $1.07 / 1.61$ & $0.83 / 1.39$ & $0.40 / 0.81$ \\
\hline Fluoride (F) & 4.27 & 4.61 & 3.33 \\
\hline Chloride (Cl) & 490 & 480 & 417 \\
\hline Bromide (Br) & 2.03 & 2.07 & 1.45 \\
\hline Nitrate $\left(\mathrm{NO}_{3}\right)$ & 0.10 & 0.24 & $<0.05$ \\
\hline Ammonium $\left(\mathrm{NH}_{4}\right)$ & 1.40 & 2.56 & 2.65 \\
\hline Silica $\left(\mathrm{SiO}_{2}\right)$ & 364 & 371 & 385 \\
\hline Boron (B) & 7.63 & 7.06 & 6.16 \\
\hline Aluminum (Al) & 2.47 & 2.24 & 1.57 \\
\hline Iron total $(\mathrm{Fe}(\mathrm{T}))$ & 1.27 & 0.944 & 1.48 \\
\hline Ferrous iron (Fe(II)) & 0.203 & 0.388 & 1.48 \\
\hline Manganese (Mn) & 0.080 & 0.066 & 0.059 \\
\hline Copper $(\mathrm{Cu})$ & 0.0006 & $<0.0005$ & $<0.0005$ \\
\hline Zinc (Zn) & 0.018 & 0.008 & 0.017 \\
\hline Cadmium (Cd) & $<0.0001$ & $<0.0001$ & $<0.0001$ \\
\hline Chromium (Cr) & $<0.0005$ & $<0.0005$ & $<0.0005$ \\
\hline Cobalt (Co) & 0.0019 & $<0.0007$ & 0.0012 \\
\hline Mercury total (Hg), ng/L & 17.0 & 35.3 & --- \\
\hline Methylmercury $\left(\mathrm{CH}_{3} \mathrm{Hg}\right), \mathrm{ng} / \mathrm{L}$ & 0.04 & 0.16 & --- \\
\hline Nickel (Ni) & $<0.0005$ & $<0.0005$ & $<0.0005$ \\
\hline Lead $(\mathrm{Pb})$ & $<0.0008$ & $<0.0008$ & $<0.0008$ \\
\hline Beryllium (Be) & 0.002 & 0.002 & 0.002 \\
\hline Vanadium (V) & $<0.005$ & $<0.005$ & $<0.005$ \\
\hline Molybdenum (Mo) & 0.048 & 0.047 & $<0.007$ \\
\hline Antimony (Sb) & 0.060 & 0.053 & 0.001 \\
\hline Selenium (Se) & 0.003 & $<0.001$ & $<0.001$ \\
\hline Arsenic (As) & 1.85 & 1.70 & 1.39 \\
\hline Arsenite $(\mathrm{As}(\mathrm{III}))$ & 0.029 & 0.058 & 0.90 \\
\hline Dissolved organic carbon (DOC) & 1.2 & 1.3 & 0.8 \\
\hline$\delta \mathrm{D}$, per mil & -131.62 & -132.49 & -138.83 \\
\hline$\delta^{18} \mathrm{O}$, per mil & -13.02 & -13.03 & -14.79 \\
\hline sum cations, meq/L & 16.9 & 17.7 & 14.1 \\
\hline sum anions, meq/L & 17.0 & 16.6 & 13.6 \\
\hline Charge imbalance, percent & -0.2 & 6.0 & 3.3 \\
\hline
\end{tabular}


Table 6. Results of water analyses for Norris Geyser Basin, 2006-2008-Continued.

\begin{tabular}{|c|c|c|c|c|c|}
\hline \multicolumn{6}{|c|}{ - } \\
\hline Location & Cistern Spring & Cistern Spring & Cistern Spring & Cistern Spring & Echinus Geyser \\
\hline Sample ID & 06WA123 & 06WA159 & 07WA116 & 08WA127 & 08WA126 \\
\hline Collection Date / Time & $5 / 16 / 0612: 20$ & $9 / 15 / 0611: 20$ & 9/13/07 13:35 & $9 / 24 / 0810: 15$ & $9 / 24 / 089: 30$ \\
\hline Temperature, ${ }^{\circ} \mathrm{C}$ & 85.7 & 84.5 & 81.1 & 79.5 & 77.1 \\
\hline pH (field / laboratory) & $5.37 / 6.76$ & $5.54 / 7.19$ & $4.51 / 4.30$ & $4.25 / 4.19$ & $3.52 / 3.37$ \\
\hline $\begin{array}{l}\text { Specific conductance } \\
\text { (field / laboratory), } \mu \mathrm{S} / \mathrm{cm}\end{array}$ & 1792 / 1934 & 1995 / 2120 & $1643 / 1708$ & $1660 / 1783$ & $1136 / 1288$ \\
\hline Eh, V & 0.057 & -0.052 & 0.019 & 0.140 & 0.480 \\
\hline Density, $\mathrm{g} / \mathrm{mL}$ at $20^{\circ} \mathrm{C}$ & 0.9993 & 0.9994 & 0.9991 & 0.9992 & 0.9989 \\
\hline Dissolved oxygen (DO), mg/L & --- & --- & --- & --- & --- \\
\hline \multicolumn{6}{|l|}{ Constituent, mg/L ${ }^{1}$} \\
\hline Calcium (Ca) & 2.13 & 2.50 & 1.80 & 1.54 & 4.28 \\
\hline Magnesium (Mg) & 0.097 & 0.100 & 0.092 & 0.046 & 0.487 \\
\hline Sodium (Na) & 335 & 353 & 318 & 280 & 176 \\
\hline Potassium (K) & 45.5 & 63.6 & 33.6 & 38.4 & 48.1 \\
\hline Strontium (Sr) & 0.008 & 0.010 & 0.006 & 0.006 & 0.007 \\
\hline Barium (Ba) & 0.018 & 0.021 & 0.013 & 0.013 & 0.055 \\
\hline Lithium (Li) & 3.52 & 4.46 & 3.08 & 3.38 & 0.874 \\
\hline Sulfate $\left(\mathrm{SO}_{4}\right)$ & 69.3 & 57.7 & 98.3 & 69.7 & 282 \\
\hline Thiosulfate $\left(\mathrm{S}_{2} \mathrm{O}_{3}\right)$ & 12.1 & 13.8 & 22.2 & --- & $<0.1$ \\
\hline Polythionate $\left(\mathrm{S}_{\mathrm{n}} \mathrm{O}_{6}\right), \mathrm{mM}$ & $<0.001$ & $<0.001$ & $<0.001$ & --- & $<0.001$ \\
\hline Hydrogen sulfide $\left(\mathrm{H}_{2} \mathrm{~S}\right)$ & 0.32 & 0.63 & 0.53 & 0.73 & 0.10 \\
\hline Alkalinity $\left(\mathrm{HCO}_{3}\right)$ & 9.1 & 12.7 & --- & --- & -- \\
\hline Acidity (free / total), mM & --- & --- & --- & --- & $0.46 / 0.96$ \\
\hline Fluoride (F) & 5.96 & 5.76 & 5.64 & 5.62 & 5.53 \\
\hline Chloride $(\mathrm{Cl})$ & 498 & 559 & 430 & 450 & 159 \\
\hline Bromide (Br) & 2.00 & 1.83 & 1.19 & 1.44 & 0.45 \\
\hline Nitrate $\left(\mathrm{NO}_{3}\right)$ & $<0.05$ & $<0.05$ & $<0.05$ & 0.01 & 0.03 \\
\hline Ammonium $\left(\mathrm{NH}_{4}\right)$ & 1.44 & 1.35 & 7.86 & 6.76 & 1.15 \\
\hline Silica $\left(\mathrm{SiO}_{2}\right)$ & 649 & 609 & 412 & 432 & 297 \\
\hline Boron (B) & 7.63 & 8.72 & 7.81 & 7.76 & 2.43 \\
\hline Aluminum (Al) & 0.11 & 0.216 & 0.13 & 0.13 & 2.22 \\
\hline Iron total $(\mathrm{Fe}(\mathrm{T}))$ & $<0.002$ & 0.009 & 0.010 & 0.024 & 2.21 \\
\hline Ferrous iron (Fe(II)) & $<0.002$ & 0.009 & 0.010 & 0.024 & 2.15 \\
\hline Manganese (Mn) & 0.039 & 0.048 & 0.059 & 0.037 & 0.255 \\
\hline Copper $(\mathrm{Cu})$ & $<0.0005$ & $<0.0005$ & $<0.0005$ & $<0.0005$ & $<0.0005$ \\
\hline Zinc (Zn) & $<0.004$ & $<0.004$ & 0.005 & $<0.004$ & 0.015 \\
\hline Cadmium (Cd) & $<0.0001$ & $<0.0001$ & $<0.0001$ & $<0.0001$ & 0.0001 \\
\hline Chromium (Cr) & $<0.0005$ & 0.0006 & $<0.0005$ & $<0.0005$ & $<0.0005$ \\
\hline Cobalt (Co) & 0.0035 & 0.0050 & $<0.0007$ & $<0.0007$ & $<0.0007$ \\
\hline Mercury total (Hg), ng/L & 132 & 19.0 & 42.0 & 340 & 36.5 \\
\hline Methylmercury $\left(\mathrm{CH}_{3} \mathrm{Hg}\right), \mathrm{ng} / \mathrm{L}$ & -- & 0.04 & 0.78 & 0.58 & --- \\
\hline Nickel (Ni) & $<0.0005$ & $<0.0005$ & $<0.0005$ & $<0.0005$ & $<0.0005$ \\
\hline Lead $(\mathrm{Pb})$ & $<0.0008$ & $<0.0008$ & $<0.0008$ & $<0.0008$ & $<0.0008$ \\
\hline Beryllium (Be) & $<0.001$ & $<0.001$ & $<0.001$ & $<0.001$ & 0.007 \\
\hline Vanadium (V) & $<0.005$ & $<0.005$ & $<0.005$ & $<0.005$ & $<0.005$ \\
\hline Molybdenum (Mo) & 0.096 & 0.125 & 0.071 & 0.103 & $<0.007$ \\
\hline Antimony (Sb) & 0.084 & 0.085 & 0.060 & 0.061 & $<0.001$ \\
\hline Selenium (Se) & $<0.001$ & $<0.001$ & $<0.001$ & $<0.001$ & $<0.001$ \\
\hline Arsenic (As) & 1.77 & 2.09 & 1.49 & 1.52 & 0.257 \\
\hline Arsenite (As(III)) & $1.45^{2}$ & $1.58^{2}$ & $1.30^{2}$ & $1.27^{2}$ & 0.061 \\
\hline Dissolved organic carbon (DOC) & 1.2 & 0.6 & 0.9 & 0.7 & 1.1 \\
\hline$\delta \mathrm{D}$, per mil & -128.57 & -128.47 & -117.94 & -121.06 & -140.67 \\
\hline$\delta^{18} 0$, per mil & -12.04 & -12.30 & -8.08 & -8.48 & -16.48 \\
\hline sum cations, meq/L & 16.4 & 17.8 & 15.7 & 14.2 & 9.7 \\
\hline sum anions, meq/L & 15.9 & 17.5 & 14.4 & 14.4 & 10.0 \\
\hline Charge imbalance, percent & 3.0 & 2.0 & 8.6 & -1.1 & -3.8 \\
\hline
\end{tabular}


Table 6. Results of water analyses for Norris Geyser Basin, 2006-2008-Continued.

\begin{tabular}{|c|c|c|c|c|c|}
\hline & 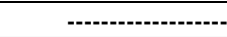 & - & --Back Basin---- & 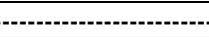 & 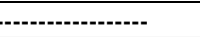 \\
\hline Location & Perpetual Spouter & Perpetual Spouter & Perpetual Spouter & Porkchop Geyser & Porkchop Geyser \\
\hline Sample ID & 06WA132 & 07WA144 & 08WA130 & 07WA143 & 08WA129 \\
\hline Collection Date / Time & $5 / 17 / 0614: 15$ & 9/18/07 15:15 & 9/24/08 13:21 & 9/18/07 14:10 & 9/24/08 12:07 \\
\hline Temperature, ${ }^{\circ} \mathrm{C}$ & 90.7 & 78.1 & 88.0 & 86.1 & 80.1 \\
\hline pH (field / laboratory) & $7.12 / 7.29$ & $7.03 / 7.36$ & $7.51 / 7.42$ & $4.52 / 4.47$ & $8.40 / 8.76$ \\
\hline \multicolumn{6}{|l|}{ Specific conductance } \\
\hline Eh, V & 0.030 & -0.102 & 0.060 & -0.059 & 0.020 \\
\hline Density, $\mathrm{g} / \mathrm{mL}$ at $20^{\circ} \mathrm{C}$ & 0.9995 & 0.9994 & 0.9993 & 0.9992 & 0.9993 \\
\hline Dissolved oxygen (DO), mg/L & --- & --- & --- & --- & --- \\
\hline \multicolumn{6}{|l|}{ Constituent, $\mathrm{mg} / \mathrm{L}^{1}$} \\
\hline Calcium (Ca) & 9.49 & 9.80 & 8.41 & 4.37 & 3.35 \\
\hline Magnesium (Mg) & 0.070 & 0.065 & 0.059 & 0.018 & 0.004 \\
\hline Sodium (Na) & 463 & 478 & 429 & 348 & 394 \\
\hline Potassium (K) & 42.9 & 45.1 & 46.1 & 53.0 & 77.7 \\
\hline Strontium (Sr) & 0.034 & 0.030 & 0.031 & 0.012 & 0.012 \\
\hline Barium (Ba) & 0.013 & 0.013 & 0.010 & 0.008 & 0.007 \\
\hline Lithium (Li) & 5.26 & 5.52 & 5.09 & 4.55 & 5.18 \\
\hline Sulfate $\left(\mathrm{SO}_{4}\right)$ & 33.2 & 41.2 & 34.8 & 51.1 & 23.7 \\
\hline Thiosulfate $\left(\mathrm{S}_{2} \mathrm{O}_{3}\right)$ & $<0.1$ & $<0.1$ & --- & 42.1 & 0.3 \\
\hline Polythionate $\left(\mathrm{S}_{\mathrm{n}} \mathrm{O}_{6}\right), \mathrm{mM}$ & $<0.001$ & $<0.001$ & --- & $<0.001$ & $<0.001$ \\
\hline Hydrogen sulfide $\left(\mathrm{H}_{2} \mathrm{~S}\right)$ & 0.019 & 0.12 & 0.04 & 1.23 & 0.11 \\
\hline Alkalinity $\left(\mathrm{HCO}_{3}\right)$ & 12.9 & 18.6 & 15.2 & $<1$ & 55.9 \\
\hline Acidity (free / total), mM & --- & --- & --- & -- & -- \\
\hline Fluoride (F) & 6.82 & 8.50 & 7.88 & 5.67 & 7.05 \\
\hline Chloride $(\mathrm{Cl})$ & 749 & 788 & 754 & 572 & 644 \\
\hline Bromide (Br) & 2.73 & 2.67 & 2.48 & 1.86 & 2.19 \\
\hline Nitrate $\left(\mathrm{NO}_{3}\right)$ & 0.26 & 0.12 & 0.27 & 0.11 & 0.24 \\
\hline Ammonium $\left(\mathrm{NH}_{4}\right)$ & 0.718 & 1.47 & 0.620 & 11.3 & 1.58 \\
\hline Silica $\left(\mathrm{SiO}_{2}\right)$ & 362 & 304 & 302 & 378 & 567 \\
\hline Boron (B) & 11.2 & 12.4 & 11.5 & 8.98 & 9.66 \\
\hline Aluminum (Al) & 0.183 & 0.165 & 0.131 & 0.041 & 0.015 \\
\hline Iron total $(\mathrm{Fe}(\mathrm{T}))$ & 0.072 & 0.108 & 0.013 & 0.030 & $<0.002$ \\
\hline Ferrous iron (Fe(II)) & 0.009 & 0.072 & 0.006 & 0.030 & $<0.002$ \\
\hline Manganese (Mn) & 0.037 & 0.040 & 0.034 & 0.033 & 0.003 \\
\hline Copper $(\mathrm{Cu})$ & $<0.0005$ & $<0.0005$ & $<0.0005$ & $<0.0005$ & $<0.0005$ \\
\hline Zinc (Zn) & $<0.004$ & $<0.004$ & $<0.004$ & 0.008 & $<0.004$ \\
\hline Cadmium (Cd) & $<0.0001$ & $<0.0001$ & $<0.0001$ & $<0.0001$ & $<0.0001$ \\
\hline Chromium (Cr) & 0.0039 & $<0.0005$ & $<0.0005$ & $<0.0005$ & $<0.0005$ \\
\hline Cobalt (Co) & 0.0092 & $<0.0007$ & $<0.0007$ & $<0.0007$ & $<0.0007$ \\
\hline Mercury total (Hg), ng/L & 22.0 & 120 & 23.2 & 3.4 & 48.5 \\
\hline Methylmercury $\left(\mathrm{CH}_{3} \mathrm{Hg}\right), \mathrm{ng} / \mathrm{L}$ & --- & 0.04 & --- & 0.06 & --- \\
\hline Nickel (Ni) & $<0.0005$ & $<0.0005$ & $<0.0005$ & $<0.0005$ & $<0.0005$ \\
\hline Lead $(\mathrm{Pb})$ & $<0.0008$ & $<0.0008$ & $<0.0008$ & $<0.0008$ & $<0.0008$ \\
\hline Beryllium (Be) & $<0.001$ & $<0.001$ & $<0.001$ & $<0.001$ & $<0.001$ \\
\hline Vanadium (V) & $<0.005$ & $<0.005$ & $<0.005$ & $<0.005$ & $<0.005$ \\
\hline Molybdenum (Mo) & 0.071 & 0.086 & 0.077 & 0.164 & 0.214 \\
\hline Antimony (Sb) & 0.125 & 0.112 & 0.161 & 0.056 & 0.147 \\
\hline Selenium (Se) & $<0.001$ & $<0.001$ & $<0.001$ & $<0.001$ & $<0.001$ \\
\hline Arsenic (As) & 3.01 & 2.70 & 2.92 & 1.79 & 2.48 \\
\hline Arsenite (As(III)) & 1.28 & 2.26 & 1.98 & 1.76 & 2.02 \\
\hline Dissolved organic carbon (DOC) & 0.7 & 0.3 & 0.9 & 0.5 & 1.0 \\
\hline$\delta \mathrm{D}$, per mil & -139.42 & -141.30 & -140.54 & -119.02 & -143.14 \\
\hline$\delta^{18} 0$, per mil & -14.85 & -14.83 & -14.93 & -7.09 & -15.00 \\
\hline sum cations, meq/L & 22.5 & 23.3 & 21.1 & 18.0 & 20.2 \\
\hline sum anions, meq/L & 22.5 & 23.9 & 22.8 & 17.5 & 20.8 \\
\hline Charge imbalance, percent & 0.1 & -2.5 & -7.7 & 3.0 & -3.3 \\
\hline
\end{tabular}


Table 6. Results of water analyses for Norris Geyser Basin, 2006-2008-Continued.

\begin{tabular}{|c|c|c|c|c|c|}
\hline & 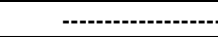 & 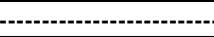 & --Back Basin---. & - & - \\
\hline Location & "Second Erupter" & "Second Erupter" & "Second Erupter" & $\begin{array}{c}\text { Tantalus Creek - } \\
\text { Back Basin Drainage }\end{array}$ & $\begin{array}{c}\text { Unnamed hot spring } \\
\text { near Perpetual } \\
\text { Spouter }\end{array}$ \\
\hline Sample ID & 06WA122 & 07WA142 & 08WA128 & 06WA134 & 06WA133 \\
\hline Collection Date / Time & 5/16/06 11:30 & 9/18/07 13:10 & 9/24/08 11:10 & $5 / 17 / 0614: 20$ & 5/17/06 16:00 \\
\hline Temperature, ${ }^{\circ} \mathrm{C}$ & 89.3 & 83.3 & 89.1 & 37.1 & 89.0 \\
\hline pH (field / laboratory) & $8.02 / 8.68$ & $6.31 / 7.64$ & $8.00 / 8.54$ & $3.13 / 3.14$ & $2.95 / 2.81$ \\
\hline Specific conductance & & & & & \\
\hline (field / laboratory), $\mu \mathrm{S} / \mathrm{cm}$ & $2230 / 2410$ & $2420 / 2530$ & $2170 / 2330$ & $2040 / 2110$ & $1380 / 1852$ \\
\hline Eh, V & 0.027 & -0.130 & 0.010 & 0.659 & 0.432 \\
\hline Density, $\mathrm{g} / \mathrm{mL}$ at $20^{\circ} \mathrm{C}$ & 0.9995 & 0.9995 & 0.9995 & 0.9992 & 0.9990 \\
\hline Dissolved oxygen (DO), mg/L & --- & --- & --- & --- & --- \\
\hline Constituent, $\mathrm{mg} / \mathrm{L}^{1}$ & & & & & \\
\hline Calcium (Ca) & 4.04 & 6.11 & 3.63 & 3.63 & 3.14 \\
\hline Magnesium (Mg) & 0.013 & 0.041 & 0.008 & 0.245 & 0.357 \\
\hline Sodium (Na) & 398 & 453 & 368 & 285 & 163 \\
\hline Potassium (K) & 71.2 & 70.1 & 76.8 & 52.4 & 59.7 \\
\hline Strontium (Sr) & 0.014 & 0.018 & 0.013 & 0.012 & 0.017 \\
\hline Barium (Ba) & 0.008 & 0.014 & 0.007 & 0.057 & 0.111 \\
\hline Lithium (Li) & 5.37 & 5.91 & 5.08 & 3.41 & 1.000 \\
\hline Sulfate $\left(\mathrm{SO}_{4}\right)$ & 21.0 & 61.4 & 23.6 & 126 & 214 \\
\hline Thiosulfate $\left(\mathrm{S}_{2} \mathrm{O}_{3}\right)$ & $<0.1$ & 14.4 & $<0.1$ & --- & $<0.1$ \\
\hline Polythionate $\left(\mathrm{S}_{\mathrm{n}} \mathrm{O}_{6}\right), \mathrm{mM}$ & $<0.001$ & $<0.001$ & $<0.001$ & --- & $<0.001$ \\
\hline Hydrogen sulfide $\left(\mathrm{H}_{2} \mathrm{~S}\right)$ & 0.13 & 0.60 & 0.36 & --- & 0.008 \\
\hline Alkalinity $\left(\mathrm{HCO}_{3}\right)$ & 55.5 & 39.8 & 54.2 & --- & -- \\
\hline Acidity (free / total), mM & --- & --- & --- & $0.87 / 1.33$ & $1.85 / 2.17$ \\
\hline Fluoride (F) & 6.66 & 7.49 & 7.02 & 4.16 & 0.746 \\
\hline Chloride (Cl) & 647 & 764 & 631 & 454 & 234 \\
\hline Bromide (Br) & 2.32 & 2.40 & 2.13 & 1.53 & 0.84 \\
\hline Nitrate $\left(\mathrm{NO}_{3}\right)$ & $<0.05$ & 0.08 & 0.33 & $<0.05$ & 0.15 \\
\hline Ammonium $\left(\mathrm{NH}_{4}\right)$ & 0.351 & 2.33 & 1.02 & 1.32 & 0.09 \\
\hline Silica $\left(\mathrm{SiO}_{2}\right)$ & 780 & 427 & 625 & 371 & 412 \\
\hline Boron (B) & 9.75 & 11.8 & 9.57 & 7.05 & 3.92 \\
\hline Aluminum (Al) & 0.011 & 0.015 & 0.014 & 2.06 & 0.57 \\
\hline Iron total $(\mathrm{Fe}(\mathrm{T}))$ & $<0.002$ & $<0.002$ & $<0.002$ & 0.875 & 5.51 \\
\hline Ferrous iron (Fe(II)) & $<0.002$ & $<0.002$ & $<0.002$ & 0.432 & 5.12 \\
\hline Manganese (Mn) & 0.006 & 0.052 & 0.006 & 0.080 & 0.112 \\
\hline Copper $(\mathrm{Cu})$ & $<0.0005$ & $<0.0005$ & 0.0604 & $<0.0005$ & $<0.0005$ \\
\hline Zinc (Zn) & $<0.004$ & $<0.004$ & $<0.004$ & 0.006 & 0.059 \\
\hline Cadmium (Cd) & $<0.0001$ & $<0.0001$ & $<0.0001$ & $<0.0001$ & $<0.0001$ \\
\hline Chromium (Cr) & 0.0008 & $<0.0005$ & $<0.0005$ & 0.0005 & 0.0006 \\
\hline Cobalt (Co) & 0.0046 & $<0.0007$ & $<0.0007$ & 0.0028 & 0.0034 \\
\hline Mercury total (Hg), ng/L & 174 & 512 & 38.7 & 41.0 & 36.0 \\
\hline Methylmercury $\left(\mathrm{CH}_{3} \mathrm{Hg}\right), \mathrm{ng} / \mathrm{L}$ & -- & 0.06 & 0.04 & -- & --- \\
\hline Nickel (Ni) & $<0.0005$ & $<0.0005$ & $<0.0005$ & $<0.0005$ & $<0.0005$ \\
\hline Lead $(\mathrm{Pb})$ & $<0.0008$ & $<0.0008$ & $<0.0008$ & $<0.0008$ & 0.0015 \\
\hline Beryllium (Be) & $<0.001$ & $<0.001$ & $<0.001$ & 0.002 & 0.004 \\
\hline Vanadium (V) & $<0.005$ & $<0.005$ & $<0.005$ & $<0.005$ & $<0.005$ \\
\hline Molybdenum (Mo) & 0.215 & 0.222 & 0.221 & 0.079 & $<0.007$ \\
\hline Antimony (Sb) & 0.141 & 0.093 & 0.177 & 0.093 & 0.023 \\
\hline Selenium (Se) & $<0.001$ & $<0.001$ & $<0.001$ & $<0.001$ & $<0.001$ \\
\hline Arsenic (As) & 2.67 & 2.68 & 2.43 & 1.82 & 0.943 \\
\hline Arsenite (As(III)) & 2.41 & 2.53 & 2.23 & 0.104 & 0.444 \\
\hline Dissolved organic carbon (DOC) & 5.0 & 0.5 & 0.4 & 1.5 & 0.7 \\
\hline$\delta \mathrm{D}$, per mil & -142.98 & -120.54 & -143.55 & -130.02 & -136.35 \\
\hline$\delta^{18} 0$, per mil & -15.54 & -9.47 & -15.64 & -12.98 & -15.38 \\
\hline sum cations, meq/L & 20.1 & 22.8 & 19.0 & 15.4 & 10.3 \\
\hline sum anions, meq/L & 20.3 & 23.9 & 19.7 & 15.4 & 10.4 \\
\hline Charge imbalance, percent & -0.9 & -4.7 & -3.6 & 0.1 & -0.3 \\
\hline
\end{tabular}


Table 6. Results of water analyses for Norris Geyser Basin, 2006-2008_Continued.

\begin{tabular}{|c|c|c|c|c|c|}
\hline & \multicolumn{3}{|c|}{ - } & \multicolumn{2}{|c|}{ 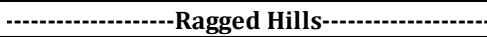 } \\
\hline Location & $\begin{array}{l}\text { Unnamed hot spring } \\
\text { near Perpetual } \\
\text { Spouter }\end{array}$ & $\begin{array}{c}\text { Unnamed hot spring, } \\
\text { North end of Elk } \\
\text { Park }\end{array}$ & $\begin{array}{c}\text { Unnamed hot spring, } \\
\text { North end of Elk } \\
\text { Park }\end{array}$ & $\begin{array}{c}\text { Appendage side } \\
\text { spring to "Lifeboat } \\
\text { Spring" }\end{array}$ & $\begin{array}{l}\text { Circular clay hot } \\
\text { spring 2m from } \\
08 W A 121\end{array}$ \\
\hline Sample ID & 07WA145 & 07WA140 & 07WA141 & 08WA124 & 08 WA123 \\
\hline Collection Date / Time & $9 / 18 / 07$ 15:55 & $9 / 18 / 079: 50$ & 9/18/07 10:40 & $9 / 23 / 0815: 40$ & 9/23/08 15:09 \\
\hline Temperature, ${ }^{\circ} \mathrm{C}$ & 93.0 & 68.3 & 91.4 & 89.3 & 63.5 \\
\hline pH (field / laboratory) & $2.92 / 2.90$ & $7.69 / 8.11$ & $4.14 / 4.10$ & $2.64 / 2.52$ & $2.80 / 2.79$ \\
\hline Specific conductance & & & & & \\
\hline (field / laboratory), $\mu \mathrm{S} / \mathrm{cm}$ & $1325 / 1776$ & $2150 / 2170$ & $2050 / 2190$ & $2020 /---$ & $840 / 1150$ \\
\hline Eh, V & 0.039 & 0.013 & -0.070 & 0.460 & 0.170 \\
\hline Density, $\mathrm{g} / \mathrm{mL}$ at $20^{\circ} \mathrm{C}$ & 0.9991 & 0.9993 & 0.9993 & --- & 0.9986 \\
\hline Dissolved oxygen (DO), mg/L & --- & --- & --- & --- & --- \\
\hline \multicolumn{6}{|l|}{ Constituent, $\mathrm{mg} / \mathrm{L}^{1}$} \\
\hline Calcium (Ca) & 3.58 & 5.99 & 6.78 & 3.03 & 0.872 \\
\hline Magnesium (Mg) & 0.360 & 0.051 & 0.109 & 0.174 & 0.059 \\
\hline Sodium (Na) & 176 & 436 & 410 & 263 & 60.0 \\
\hline Potassium (K) & 51.2 & 21.3 & 25.4 & 42.7 & 15.5 \\
\hline Strontium (Sr) & 0.016 & 0.014 & 0.016 & 0.010 & 0.004 \\
\hline Barium (Ba) & 0.113 & 0.009 & 0.035 & 0.069 & 0.081 \\
\hline Lithium (Li) & 0.936 & 2.96 & 3.16 & 3.02 & 0.618 \\
\hline Sulfate $\left(\mathrm{SO}_{4}\right)$ & 224 & 30.5 & 85.6 & 316 & 119 \\
\hline Thiosulfate $\left(\mathrm{S}_{2} \mathrm{O}_{3}\right)$ & $<0.1$ & $<0.1$ & 0.5 & --- & --- \\
\hline Polythionate $\left(\mathrm{S}_{\mathrm{n}} \mathrm{O}_{6}\right), \mathrm{mM}$ & $<0.001$ & $<0.001$ & $<0.001$ & --- & --- \\
\hline Hydrogen sulfide $\left(\mathrm{H}_{2} \mathrm{~S}\right)$ & 0.03 & 0.007 & 0.44 & --- & --- \\
\hline Alkalinity $\left(\mathrm{HCO}_{3}\right)$ & -- & 92.8 & --- & --- & -- \\
\hline Acidity (free / total), mM & $1.97 / 2.13$ & --- & --- & $3.13 / 4.36$ & $1.51 / 1.95$ \\
\hline Fluoride (F) & 0.900 & 13.2 & 10.1 & 4.76 & 0.659 \\
\hline Chloride (Cl) & 254 & 613 & 619 & 442 & 110 \\
\hline Bromide (Br) & 0.86 & 2.07 & 1.99 & 1.27 & 0.32 \\
\hline Nitrate $\left(\mathrm{NO}_{3}\right)$ & 0.12 & 0.11 & 0.10 & 0.24 & $<0.05$ \\
\hline Ammonium $\left(\mathrm{NH}_{4}\right)$ & 3.24 & 4.54 & 1.71 & --- & 0.200 \\
\hline Silica $\left(\mathrm{SiO}_{2}\right)$ & 426 & 304 & 303 & 238 & 211 \\
\hline Boron (B) & 4.00 & 9.25 & 9.38 & 5.71 & 1.54 \\
\hline Aluminum (Al) & 0.50 & 0.153 & 1.57 & 8.94 & 0.92 \\
\hline Iron total $(\mathrm{Fe}(\mathrm{T}))$ & 6.17 & 0.005 & 0.181 & 2.79 & 1.23 \\
\hline Ferrous iron (Fe(II)) & 6.17 & 0.005 & 0.181 & 2.61 & 1.21 \\
\hline Manganese (Mn) & 0.118 & 0.044 & 0.269 & 0.023 & 0.014 \\
\hline Copper $(\mathrm{Cu})$ & 0.0006 & $<0.0005$ & $<0.0005$ & 0.0012 & $<0.0005$ \\
\hline Zinc $(\mathrm{Zn})$ & 0.061 & $<0.004$ & $<0.004$ & 0.042 & 0.006 \\
\hline Cadmium (Cd) & $<0.0001$ & $<0.0001$ & $<0.0001$ & 0.0001 & $<0.0001$ \\
\hline Chromium (Cr) & $<0.0005$ & $<0.0005$ & 0.0009 & 0.0014 & $<0.0005$ \\
\hline Cobalt (Co) & $<0.0007$ & $<0.0007$ & $<0.0007$ & $<0.0007$ & $<0.0007$ \\
\hline Mercury total (Hg), ng/L & 25.0 & 42.0 & 300 & --- & 110 \\
\hline Methylmercury $\left(\mathrm{CH}_{3} \mathrm{Hg}\right), \mathrm{ng} / \mathrm{L}$ & 0.06 & 0.10 & 0.22 & --- & --- \\
\hline Nickel (Ni) & $<0.0005$ & $<0.0005$ & $<0.0005$ & 0.0009 & $<0.0005$ \\
\hline Lead $(\mathrm{Pb})$ & 0.0012 & $<0.0008$ & $<0.0008$ & $<0.0008$ & $<0.0008$ \\
\hline Beryllium (Be) & 0.004 & $<0.001$ & 0.001 & $<0.001$ & $<0.001$ \\
\hline Vanadium (V) & $<0.005$ & $<0.005$ & $<0.005$ & $<0.005$ & $<0.005$ \\
\hline Molybdenum (Mo) & $<0.007$ & 0.223 & 0.223 & 0.030 & $<0.007$ \\
\hline Antimony (Sb) & 0.034 & 0.082 & 0.075 & 0.142 & 0.018 \\
\hline Selenium (Se) & $<0.001$ & $<0.001$ & $<0.001$ & $<0.001$ & $<0.001$ \\
\hline Arsenic (As) & 0.949 & 1.76 & 1.86 & 1.75 & 5.54 \\
\hline Arsenite (As(III)) & 0.579 & 0.022 & 1.86 & 0.470 & 2.87 \\
\hline Dissolved organic carbon (DOC) & 0.5 & 0.6 & 0.5 & --- & 0.7 \\
\hline$\delta \mathrm{D}$, per mil & -136.49 & -141.73 & -129.96 & -132.11 & -147.20 \\
\hline$\delta^{18} 0$, per mil & -15.23 & -14.49 & -11.16 & -10.25 & -15.36 \\
\hline sum cations, meq/L & 11.0 & 20.4 & 19.4 & 16.4 & 5.0 \\
\hline sum anions, meq/L & 11.0 & 20.2 & 19.5 & 17.5 & 5.3 \\
\hline Charge imbalance, percent & -0.2 & 1.1 & -0.2 & -6.4 & -5.1 \\
\hline
\end{tabular}


Table 6. Results of water analyses for Norris Geyser Basin, 2006-2008-Continued.

\begin{tabular}{|c|c|c|c|c|c|}
\hline \multirow[b]{2}{*}{ Location } & \multicolumn{5}{|c|}{ 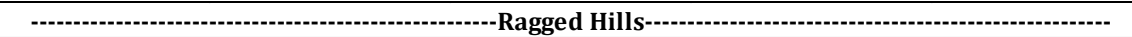 } \\
\hline & $\begin{array}{l}\text { Crystal Spring, } \\
\text { eastern side }\end{array}$ & $\begin{array}{l}\text { Crystal Spring, } \\
\text { eastern side }\end{array}$ & $\begin{array}{c}\text { Crystal Spring, } \\
\text { western side }\end{array}$ & $\begin{array}{l}\text { Crystal Spring, } \\
\text { western side }\end{array}$ & $\begin{array}{c}\text { Crystal Spring, } \\
\text { western side }\end{array}$ \\
\hline Sample ID & 06WA112 & 07WA147 & 06WA111 & 07WA146 & 08WA120 \\
\hline Collection Date / Time & $5 / 13 / 0618: 00$ & 9/18/07 17:05 & $5 / 13 / 0617: 35$ & 9/18/07 16:40 & 9/23/08 12:30 \\
\hline Temperature, ${ }^{\circ} \mathrm{C}$ & 80.6 & 77.0 & 80.3 & 82.9 & 80.8 \\
\hline pH (field / laboratory) & $5.55 / 6.25$ & $1.97 / 1.96$ & $3.77 / 3.67$ & $1.98 / 1.94$ & $2.29 / 2.15$ \\
\hline $\begin{array}{l}\text { Specific conductance } \\
\text { (field / laboratory), } \mu \mathrm{S} / \mathrm{cm}\end{array}$ & $2320 / 2490$ & $3660 / 6550$ & $2360 / 2550$ & 3380 / 6500 & $2630 / 4340$ \\
\hline Eh, V & 0.047 & 0.500 & 0.143 & 0.180 & -0.140 \\
\hline Density, $\mathrm{g} / \mathrm{mL}$ at $20^{\circ} \mathrm{C}$ & 0.9994 & 0.9996 & 0.9994 & 0.9995 & 0.9994 \\
\hline Dissolved oxygen (DO), $\mathrm{mg} / \mathrm{L}$ & --- & --- & --- & --- & --- \\
\hline \multicolumn{6}{|l|}{ Constituent, $\mathrm{mg} / \mathrm{L}^{1}$} \\
\hline Calcium (Ca) & 6.25 & 2.54 & 6.08 & 2.15 & 4.41 \\
\hline Magnesium (Mg) & 0.005 & 0.212 & 0.005 & 0.212 & 0.323 \\
\hline Sodium (Na) & 448 & 98.4 & 429 & 84.9 & 215 \\
\hline Potassium (K) & 31.4 & 13.4 & 30.5 & 12.3 & 23.0 \\
\hline Strontium (Sr) & 0.014 & 0.010 & 0.013 & 0.010 & 0.017 \\
\hline Barium (Ba) & 0.007 & 0.058 & 0.009 & 0.054 & 0.045 \\
\hline Lithium (Li) & 4.92 & 1.04 & 4.74 & 0.820 & 2.42 \\
\hline Sulfate $\left(\mathrm{SO}_{4}\right)$ & 30.1 & 1180 & 51.1 & 1270 & 691 \\
\hline Thiosulfate $\left(\mathrm{S}_{2} \mathrm{O}_{3}\right)$ & 2.3 & $<0.1$ & 1.6 & $<0.1$ & --- \\
\hline Polythionate $\left(\mathrm{S}_{\mathrm{n}} \mathrm{O}_{6}\right), \mathrm{mM}$ & $<0.001$ & $<0.001$ & $<0.001$ & $<0.001$ & --- \\
\hline Hydrogen sulfide $\left(\mathrm{H}_{2} \mathrm{~S}\right)$ & 0.14 & 0.04 & 0.09 & 0.17 & 0.12 \\
\hline Alkalinity $\left(\mathrm{HCO}_{3}\right)$ & 6.1 & -- & -- & -- & -- \\
\hline Acidity (free / total), mM & --- & $20.5 / 23.5$ & $0.29 / 0.52$ & $20.7 / 23.3$ & $7.67 / 9.93$ \\
\hline Fluoride (F) & 6.22 & 1.17 & 5.68 & 1.15 & 2.55 \\
\hline Chloride $(\mathrm{Cl})$ & 688 & 138 & 674 & 103 & 287 \\
\hline Bromide (Br) & 2.95 & 0.49 & 2.36 & 0.47 & 1.01 \\
\hline Nitrate $\left(\mathrm{NO}_{3}\right)$ & $<0.05$ & 0.09 & $<0.05$ & 0.09 & 0.14 \\
\hline Ammonium $\left(\mathrm{NH}_{4}\right)$ & 1.19 & 14.8 & 1.39 & 14.6 & 14.8 \\
\hline Silica $\left(\mathrm{SiO}_{2}\right)$ & 181 & 259 & 384 & 228 & 217 \\
\hline Boron (B) & 10.2 & 2.37 & 9.78 & 1.90 & 4.56 \\
\hline Aluminum (Al) & 0.24 & 25.7 & 0.67 & 22.8 & 14.2 \\
\hline Iron total $(\mathrm{Fe}(\mathrm{T}))$ & 0.020 & 4.23 & 0.034 & 3.10 & 2.10 \\
\hline Ferrous iron (Fe(II)) & 0.019 & 2.01 & 0.034 & 2.94 & 2.03 \\
\hline Manganese (Mn) & 0.004 & 0.040 & 0.004 & 0.037 & 0.046 \\
\hline Copper $(\mathrm{Cu})$ & $<0.0005$ & $<0.0005$ & $<0.0005$ & $<0.0005$ & $<0.0005$ \\
\hline Zinc (Zn) & $<0.004$ & 0.012 & $<0.004$ & 0.015 & 0.021 \\
\hline Cadmium (Cd) & $<0.0001$ & $<0.0001$ & $<0.0001$ & $<0.0001$ & 0.0005 \\
\hline Chromium (Cr) & $<0.0005$ & 0.0032 & $<0.0005$ & 0.0042 & 0.0016 \\
\hline Cobalt (Co) & 0.0023 & $<0.0007$ & 0.0020 & $<0.0007$ & $<0.0007$ \\
\hline Mercury total (Hg), ng/L & 139 & 270 & 97.0 & 470 & 350 \\
\hline Methylmercury $\left(\mathrm{CH}_{3} \mathrm{Hg}\right), \mathrm{ng} / \mathrm{L}$ & --- & 0.54 & --- & 1.70 & 1.60 \\
\hline Nickel (Ni) & $<0.0005$ & $<0.0005$ & $<0.0005$ & $<0.0005$ & $<0.0005$ \\
\hline Lead $(\mathrm{Pb})$ & $<0.0008$ & 0.0069 & $<0.0008$ & 0.0078 & 0.0044 \\
\hline Beryllium (Be) & $<0.001$ & $<0.001$ & $<0.001$ & $<0.001$ & $<0.001$ \\
\hline Vanadium (V) & $<0.005$ & $<0.005$ & $<0.005$ & $<0.005$ & $<0.005$ \\
\hline Molybdenum (Mo) & 0.209 & 0.127 & 0.203 & 0.072 & 0.045 \\
\hline Antimony (Sb) & 0.180 & 0.146 & 0.141 & 0.107 & 0.077 \\
\hline Selenium (Se) & $<0.001$ & $<0.001$ & $<0.001$ & $<0.001$ & $<0.001$ \\
\hline Arsenic (As) & 2.78 & 0.708 & 2.69 & 0.701 & 1.27 \\
\hline Arsenite (As(III)) & $2.39^{2}$ & 0.074 & $2.46^{2}$ & 0.498 & 0.597 \\
\hline Dissolved organic carbon (DOC) & 0.4 & 2.9 & 0.6 & 3.8 & 9.8 \\
\hline$\delta \mathrm{D}$, per mil & -135.49 & -115.91 & -131.68 & -117.42 & -119.35 \\
\hline$\delta^{18} 0$, per mil & -12.94 & -6.30 & -11.01 & -7.61 & -7.57 \\
\hline sum cations, meq/L & 21.4 & 19.4 & 20.7 & 18.3 & 18.0 \\
\hline sum anions, meq/L & 20.5 & 18.9 & 20.2 & 18.8 & 17.9 \\
\hline Charge imbalance, percent & 4.4 & 2.6 & 2.4 & -2.3 & 0.5 \\
\hline
\end{tabular}


Table 6. Results of water analyses for Norris Geyser Basin, 2006-2008-Continued.

\begin{tabular}{|c|c|c|c|c|c|}
\hline & \multicolumn{5}{|c|}{ 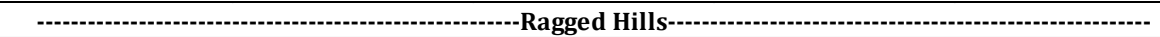 } \\
\hline Location & $\begin{array}{c}\text { The Gap northeast } \\
\text { outflow }\end{array}$ & $\begin{array}{c}\text { The Gap southwest } \\
\text { outflow }\end{array}$ & $\begin{array}{l}\text { Hot Spring next to } \\
\text { "Orpiment Puddle 2" }\end{array}$ & "Kaolin Spring" & "Kaolin Spring" \\
\hline Sample ID & 06WA121 & 06WA120 & 07WA107 & 07WA106 & 08WA131 \\
\hline Collection Date / Time & 5/15/06 13:45 & 5/15/06 13:00 & $9 / 10 / 0716: 30$ & 9/10/07 14:30 & 9/24/08 14:00 \\
\hline Temperature, ${ }^{\circ} \mathrm{C}$ & 42.0 & 36.8 & 90.0 & 73.8 & 70.0 \\
\hline pH (field / laboratory) & $3.32 / 3.24$ & $3.08 / 3.06$ & $3.12 / 3.00$ & $2.37 / 2.41$ & $2.54 / 2.51$ \\
\hline $\begin{array}{l}\text { Specific conductance } \\
\text { (field / laboratory), } \mu \mathrm{S} / \mathrm{cm}\end{array}$ & $2280 / 2350$ & $1815 / 1880$ & $1228 / 1502$ & $1442 / 2430$ & $690 / 1667$ \\
\hline Eh, V & 0.635 & 0.694 & 0.124 & 0.093 & 0.500 \\
\hline Density, $\mathrm{g} / \mathrm{mL}$ at $20^{\circ} \mathrm{C}$ & 0.9995 & 0.9991 & 0.9989 & 0.9988 & 0.9987 \\
\hline Dissolved oxygen (DO), mg/L & 5.0 & --- & --- & --- & --- \\
\hline \multicolumn{6}{|l|}{ Constituent, $\mathrm{mg} / \mathrm{L}^{1}$} \\
\hline Calcium (Ca) & 5.52 & 3.53 & 3.10 & 2.33 & 3.09 \\
\hline Magnesium (Mg) & 0.154 & 0.381 & 0.065 & 0.480 & 0.612 \\
\hline Sodium (Na) & 341 & 254 & 195 & 37.6 & 43.5 \\
\hline Potassium (K) & 42.5 & 28.3 & 22.6 & 10.9 & 16.9 \\
\hline Strontium (Sr) & 0.017 & 0.015 & 0.014 & 0.008 & 0.013 \\
\hline Barium (Ba) & 0.094 & 0.118 & 0.162 & 0.035 & 0.046 \\
\hline Lithium (Li) & 4.13 & 2.09 & 2.32 & 0.325 & 0.378 \\
\hline Sulfate $\left(\mathrm{SO}_{4}\right)$ & 105 & 115 & 98.0 & 398 & 278 \\
\hline Thiosulfate $\left(\mathrm{S}_{2} \mathrm{O}_{3}\right)$ & --- & --- & $<0.1$ & $<0.1$ & --- \\
\hline Polythionate $\left(\mathrm{S}_{\mathrm{n}} \mathrm{O}_{6}\right), \mathrm{mM}$ & --- & --- & $<0.001$ & $<0.001$ & --- \\
\hline Hydrogen sulfide $\left(\mathrm{H}_{2} \mathrm{~S}\right)$ & --- & --- & 0.04 & 0.54 & 0.001 \\
\hline Alkalinity $\left(\mathrm{HCO}_{3}\right)$ & -- & -- & --- & --- & --- \\
\hline Acidity (free / total), mM & $0.71 / 1.08$ & $1.01 / 1.51$ & $1.13 / 1.66$ & $6.40 / 7.65$ & $3.06 / 4.28$ \\
\hline Fluoride (F) & 4.79 & 4.10 & 3.09 & 0.550 & 0.480 \\
\hline Chloride (Cl) & 561 & 361 & 305 & 46.5 & 70.0 \\
\hline Bromide (Br) & 1.91 & 1.24 & 1.02 & 0.15 & 0.22 \\
\hline Nitrate $\left(\mathrm{NO}_{3}\right)$ & $<0.05$ & 0.08 & 0.06 & $<0.05$ & 0.24 \\
\hline Ammonium $\left(\mathrm{NH}_{4}\right)$ & 1.04 & 0.930 & $<0.07$ & 0.850 & 1.10 \\
\hline Silica $\left(\mathrm{SiO}_{2}\right)$ & 399 & 302 & 258 & 239 & 241 \\
\hline Boron (B) & 7.78 & 5.49 & 4.52 & 0.761 & 1.04 \\
\hline Aluminum (Al) & 1.59 & 2.52 & 2.92 & 9.30 & 7.09 \\
\hline Iron total $(\mathrm{Fe}(\mathrm{T}))$ & 1.02 & 3.04 & 5.04 & 12.9 & 8.55 \\
\hline Ferrous iron (Fe(II)) & 0.402 & 1.23 & 4.97 & 12.9 & 8.55 \\
\hline Manganese (Mn) & 0.034 & 0.077 & 0.015 & 0.065 & 0.086 \\
\hline Copper $(\mathrm{Cu})$ & $<0.0005$ & $<0.0005$ & 0.0074 & 0.0018 & $<0.0005$ \\
\hline Zinc (Zn) & 0.012 & 0.016 & 0.010 & 0.048 & 0.031 \\
\hline Cadmium (Cd) & $<0.0001$ & $<0.0001$ & 0.0001 & 0.0002 & $<0.0001$ \\
\hline Chromium (Cr) & $<0.0005$ & $<0.0005$ & $<0.0005$ & 0.0011 & 0.0006 \\
\hline Cobalt (Co) & 0.0029 & 0.0017 & $<0.0007$ & $<0.0007$ & $<0.0007$ \\
\hline Mercury total (Hg), ng/L & 89.0 & 26.0 & 260 & 540 & 68.1 \\
\hline Methylmercury $\left(\mathrm{CH}_{3} \mathrm{Hg}\right), \mathrm{ng} / \mathrm{L}$ & --- & --- & 0.19 & 0.40 & 2.60 \\
\hline Nickel (Ni) & $<0.0005$ & $<0.0005$ & $<0.0005$ & 0.0067 & $<0.0005$ \\
\hline Lead $(\mathrm{Pb})$ & $<0.0008$ & $<0.0008$ & 0.0018 & $<0.0008$ & $<0.0008$ \\
\hline Beryllium (Be) & 0.002 & 0.002 & $<0.001$ & 0.002 & 0.002 \\
\hline Vanadium (V) & $<0.005$ & $<0.005$ & $<0.005$ & 0.006 & $<0.005$ \\
\hline Molybdenum (Mo) & 0.101 & $<0.007$ & $<0.007$ & $<0.007$ & $<0.007$ \\
\hline Antimony (Sb) & 0.105 & 0.037 & 0.095 & 0.021 & 0.009 \\
\hline Selenium (Se) & $<0.001$ & 0.001 & $<0.001$ & $<0.001$ & $<0.001$ \\
\hline Arsenic (As) & 1.77 & 0.874 & 11.2 & 0.295 & 0.251 \\
\hline Arsenite (As(III)) & 0.106 & 0.049 & 11.2 & $0.190^{2}$ & $0.188^{2}$ \\
\hline Dissolved organic carbon (DOC) & 0.8 & 0.9 & 1.5 & 2.8 & 2.4 \\
\hline$\delta \mathrm{D}$, per mil & -133.18 & -138.80 & -132.40 & -125.11 & -127.20 \\
\hline$\delta^{18} 0$, per mil & -12.68 & -14.82 & -12.03 & -7.92 & -8.92 \\
\hline sum cations, meq/L & 17.4 & 13.4 & 10.7 & 7.7 & 6.4 \\
\hline sum anions, meq/L & 18.0 & 12.5 & 10.4 & 7.0 & 6.3 \\
\hline Charge imbalance, percent & -3.4 & 6.8 & 3.1 & 9.5 & 2.6 \\
\hline
\end{tabular}


Table 6. Results of water analyses for Norris Geyser Basin, 2006-2008-Continued.

\begin{tabular}{|c|c|c|c|c|c|}
\hline & 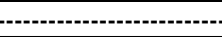 & 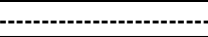 & ----Ragged Hills---. & 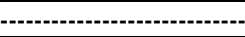 & 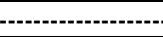 \\
\hline Location & "Lifeboat Spring" & "Lifeboat Spring" & "Lifeboat Spring" & "Orpiment Puddle 3" & $\begin{array}{c}\text { "Persnickety } \\
\text { Geyser" }\end{array}$ \\
\hline Sample ID & 06WA118 & 07WA115 & 08WA125 & 06WA110 & 06WA119 \\
\hline Collection Date / Time & 5/15/06 12:00 & 9/13/07 11:30 & 9/23/08 16:05 & 5/13/06 17:00 & $5 / 15 / 0612: 10$ \\
\hline Temperature, ${ }^{\circ} \mathrm{C}$ & 70.4 & 69.4 & 60.1 & 28.7 & 87.1 \\
\hline pH (field / laboratory) & $3.58 / 3.49$ & $2.48 / 2.39$ & $3.23 / 3.23$ & $2.43 / 2.40$ & $3.81 / 3.57$ \\
\hline Specific conductance & & & & & \\
\hline (field / laboratory), $\mu \mathrm{S} / \mathrm{cm}$ & $2170 / 2320$ & $2550 / 3330$ & $2230 / 2380$ & $2170 / 2360$ & $2060 / 2240$ \\
\hline Eh, V & --- & 0.124 & 0.620 & 0.273 & --- \\
\hline Density, $\mathrm{g} / \mathrm{mL}$ at $20^{\circ} \mathrm{C}$ & --- & 0.9994 & 0.9995 & 0.9987 & --- \\
\hline Dissolved oxygen (DO), mg/L & --- & --- & --- & --- & --- \\
\hline Constituent, $\mathrm{mg} / \mathrm{L}^{1}$ & & & & & \\
\hline Calcium (Ca) & 5.41 & 4.19 & 5.27 & 1.25 & 5.54 \\
\hline Magnesium (Mg) & 0.116 & 0.264 & 0.125 & 0.143 & 0.133 \\
\hline Sodium (Na) & 365 & 282 & 376 & 75.2 & 353 \\
\hline Potassium (K) & 47.5 & 35.9 & 58.7 & 18.0 & 49.1 \\
\hline Strontium (Sr) & 0.014 & 0.014 & 0.014 & 0.009 & 0.017 \\
\hline Barium (Ba) & 0.054 & 0.128 & 0.047 & 0.077 & 0.057 \\
\hline Lithium (Li) & 4.48 & 2.86 & 4.65 & 0.803 & 4.25 \\
\hline Sulfate $\left(\mathrm{SO}_{4}\right)$ & 70.8 & 405 & 100.0 & 307 & 84.4 \\
\hline Thiosulfate $\left(\mathrm{S}_{2} \mathrm{O}_{3}\right)$ & --- & 1.2 & --- & 0.9 & --- \\
\hline Polythionate $\left(\mathrm{S}_{\mathrm{n}} \mathrm{O}_{6}\right), \mathrm{mM}$ & --- & $<0.001$ & --- & 0.003 & --- \\
\hline Hydrogen sulfide $\left(\mathrm{H}_{2} \mathrm{~S}\right)$ & --- & 0.08 & --- & 1.80 & --- \\
\hline Alkalinity $\left(\mathrm{HCO}_{3}\right)$ & --- & -- & -- & -- & -- \\
\hline Acidity (free / total), mM & --- & $5.81 / 6.98$ & $0.40 / 0.90$ & $4.85 / 5.72$ & --- \\
\hline Fluoride (F) & 6.30 & 3.36 & 5.40 & 0.817 & 5.86 \\
\hline Chloride (Cl) & 598 & 412 & 631 & 118 & 561 \\
\hline Bromide (Br) & 1.99 & 1.09 & 3.17 & 0.38 & 1.80 \\
\hline Nitrate $\left(\mathrm{NO}_{3}\right)$ & $<0.05$ & $<0.05$ & 1.70 & $<0.05$ & $<0.05$ \\
\hline Ammonium $\left(\mathrm{NH}_{4}\right)$ & --- & 1.80 & 0.890 & $<0.07$ & --- \\
\hline Silica $\left(\mathrm{SiO}_{2}\right)$ & 464 & 369 & 452 & 318 & 436 \\
\hline Boron (B) & 8.26 & 5.98 & 8.58 & 1.62 & 7.74 \\
\hline Aluminum (Al) & 0.98 & 2.86 & 2.45 & 2.15 & 1.10 \\
\hline Iron total $(\mathrm{Fe}(\mathrm{T}))$ & 0.719 & 37.1 & 1.24 & 16.7 & 0.882 \\
\hline Ferrous iron (Fe(II)) & 0.363 & 37.1 & 0.940 & 16.7 & 0.698 \\
\hline Manganese (Mn) & 0.023 & 0.054 & 0.026 & 0.020 & 0.031 \\
\hline Copper $(\mathrm{Cu})$ & $<0.0005$ & 0.0007 & 0.0007 & $<0.0005$ & 0.0017 \\
\hline Zinc (Zn) & 0.095 & 0.034 & 0.008 & 0.023 & 0.400 \\
\hline Cadmium (Cd) & $<0.0001$ & $<0.0001$ & $<0.0001$ & $<0.0001$ & $<0.0001$ \\
\hline Chromium (Cr) & $<0.0005$ & $<0.0005$ & $<0.0005$ & 0.0012 & $<0.0005$ \\
\hline Cobalt (Co) & 0.0031 & $<0.0007$ & $<0.0007$ & 0.0013 & 0.0027 \\
\hline Mercury total (Hg), ng/L & --- & 94.0 & 19.8 & 223 & --- \\
\hline Methylmercury $\left(\mathrm{CH}_{3} \mathrm{Hg}\right), \mathrm{ng} / \mathrm{L}$ & -- & 0.59 & 0.16 & --- & --- \\
\hline Nickel (Ni) & 0.0007 & $<0.0005$ & $<0.0005$ & $<0.0005$ & $<0.0005$ \\
\hline Lead $(\mathrm{Pb})$ & $<0.0008$ & 0.0015 & $<0.0008$ & 0.0011 & 0.0009 \\
\hline Beryllium (Be) & 0.001 & 0.002 & $<0.001$ & $<0.001$ & 0.002 \\
\hline Vanadium (V) & $<0.005$ & $<0.005$ & $<0.005$ & $<0.005$ & $<0.005$ \\
\hline Molybdenum (Mo) & 0.131 & 0.265 & 0.130 & $<0.007$ & 0.123 \\
\hline Antimony (Sb) & 0.057 & 0.062 & 0.141 & 0.003 & 0.051 \\
\hline Selenium (Se) & $<0.001$ & $<0.001$ & $<0.001$ & 0.001 & $<0.001$ \\
\hline Arsenic (As) & 1.32 & 14.6 & 1.40 & 1.75 & 1.65 \\
\hline Arsenite (As(III)) & $0.087^{2}$ & 1.95 & 0.026 & $0.746^{2}$ & $1.03^{2}$ \\
\hline Dissolved organic carbon (DOC) & --- & 1.6 & 1.2 & 2.3 & --- \\
\hline$\delta \mathrm{D}$, per mil & --- & -127.47 & -136.98 & -121.62 & --- \\
\hline$\delta^{18} 0$, per mil & --- & -10.97 & -13.40 & -11.34 & --- \\
\hline sum cations, meq/L & 18.3 & 18.8 & 19.7 & 8.6 & 17.7 \\
\hline sum anions, meq/L & 18.4 & 18.1 & 19.9 & 8.8 & 17.6 \\
\hline Charge imbalance, percent & -0.5 & 3.6 & -1.1 & -2.4 & 0.4 \\
\hline
\end{tabular}


Table 6. Results of water analyses for Norris Geyser Basin, 2006-2008-Continued.

\begin{tabular}{|c|c|c|c|c|c|}
\hline \multirow[b]{2}{*}{ Location } & \multicolumn{5}{|c|}{ 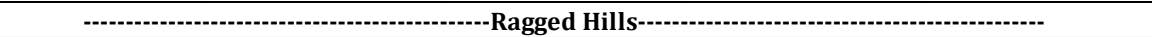 } \\
\hline & $\begin{array}{l}\text { "Persnickety } \\
\text { Geyser" }\end{array}$ & "Succession Spring" & $\begin{array}{l}\text { "Succession Spring" } \\
\text { drainage channel }\end{array}$ & $\begin{array}{l}\text { "Succession Spring" } \\
\text { drainage channel }\end{array}$ & $\begin{array}{l}\text { "Succession Spring" } \\
\text { drainage channel }\end{array}$ \\
\hline Sample ID & 07WA105 & 06WA126 & 06WA127 & 06WA128 & 06WA129 \\
\hline Collection Date / Time & 9/10/07 12:30 & $5 / 17 / 0610: 47$ & $5 / 17 / 0611: 20$ & $5 / 17 / 060: 00$ & 5/17/06 12:35 \\
\hline Temperature, ${ }^{\circ} \mathrm{C}$ & 89.0 & 65.4 & 59.4 & 50.7 & 41.2 \\
\hline pH (field / laboratory) & $3.39 / 3.17$ & $3.12 / 3.00$ & $3.02 / 3.04$ & $2.94 / 3.01$ & $2.87 / 3.00$ \\
\hline $\begin{array}{l}\text { Specific conductance } \\
\text { (field / laboratory), } \mu \mathrm{S} / \mathrm{cm}\end{array}$ & $1938 / 2220$ & $1816 / 2040$ & $1858 / 2030$ & $1830 / 2070$ & $2020 / 2130$ \\
\hline Eh, V & -0.017 & -- & --- & -- & -- \\
\hline Density, $\mathrm{g} / \mathrm{mL}$ at $20^{\circ} \mathrm{C}$ & 0.9993 & 0.9989 & 0.9991 & 0.9990 & 0.9991 \\
\hline Dissolved oxygen (DO), mg/L & --- & --- & --- & --- & --- \\
\hline \multicolumn{6}{|l|}{ Constituent, $\mathrm{mg} / \mathrm{L}^{1}$} \\
\hline Calcium (Ca) & 6.04 & 4.52 & 4.47 & 4.52 & 4.63 \\
\hline Magnesium (Mg) & 0.171 & 0.282 & 0.281 & 0.282 & 0.291 \\
\hline Sodium (Na) & 357 & 261 & 264 & 265 & 272 \\
\hline Potassium (K) & 44.0 & 30.0 & 28.8 & 29.0 & 29.4 \\
\hline Strontium (Sr) & 0.018 & 0.014 & 0.014 & 0.014 & 0.014 \\
\hline Barium (Ba) & 0.063 & 0.105 & 0.103 & 0.104 & 0.106 \\
\hline Lithium (Li) & 4.25 & 2.69 & 2.73 & 2.73 & 2.75 \\
\hline Sulfate $\left(\mathrm{SO}_{4}\right)$ & 145 & 112 & 111 & 112 & 116 \\
\hline Thiosulfate $\left(\mathrm{S}_{2} \mathrm{O}_{3}\right)$ & $<0.1$ & $<0.1$ & $<0.1$ & $<0.1$ & $<0.1$ \\
\hline Polythionate $\left(\mathrm{S}_{\mathrm{n}} \mathrm{O}_{6}\right), \mathrm{mM}$ & $<0.001$ & $<0.001$ & $<0.001$ & $<0.001$ & $<0.001$ \\
\hline Hydrogen sulfide $\left(\mathrm{H}_{2} \mathrm{~S}\right)$ & 0.73 & 2.00 & 0.10 & --- & --- \\
\hline Alkalinity $\left(\mathrm{HCO}_{3}\right)$ & -- & --- & --- & -- & -- \\
\hline Acidity (free / total), mM & $0.76 / 1.09$ & $1.01 / 1.86$ & $0.99 / 1.70$ & $1.08 / 1.76$ & $1.13 / 1.76$ \\
\hline Fluoride (F) & 5.09 & 2.72 & 2.74 & 2.83 & 2.84 \\
\hline Chloride (Cl) & 520 & 415 & 425 & 431 & 445 \\
\hline Bromide $(\mathrm{Br})$ & 1.82 & 1.45 & 1.56 & 1.54 & 1.57 \\
\hline Nitrate $\left(\mathrm{NO}_{3}\right)$ & $<0.05$ & 0.08 & 0.10 & $<0.05$ & $<0.05$ \\
\hline Ammonium $\left(\mathrm{NH}_{4}\right)$ & 1.72 & 0.830 & 0.946 & 0.771 & 0.690 \\
\hline Silica $\left(\mathrm{SiO}_{2}\right)$ & 430 & 280 & 377 & 243 & 245 \\
\hline Boron (B) & 8.21 & 6.13 & 6.22 & 6.22 & 6.55 \\
\hline Aluminum (Al) & 1.68 & 3.60 & 3.60 & 3.66 & 3.66 \\
\hline Iron total $(\mathrm{Fe}(\mathrm{T}))$ & 2.95 & 0.952 & 0.960 & 0.923 & 0.880 \\
\hline Ferrous iron (Fe(II)) & 2.95 & 0.946 & 0.942 & 0.716 & 0.484 \\
\hline Manganese (Mn) & 0.041 & 0.040 & 0.041 & 0.041 & 0.042 \\
\hline Copper $(\mathrm{Cu})$ & $<0.0005$ & $<0.0005$ & $<0.0005$ & $<0.0005$ & $<0.0005$ \\
\hline Zinc (Zn) & 0.020 & 0.010 & 0.009 & 0.008 & 0.010 \\
\hline Cadmium (Cd) & $<0.0001$ & $<0.0001$ & $<0.0001$ & $<0.0001$ & $<0.0001$ \\
\hline Chromium (Cr) & 0.0005 & $<0.0005$ & $<0.0005$ & $<0.0005$ & $<0.0005$ \\
\hline Cobalt (Co) & $<0.0007$ & 0.0008 & 0.0007 & 0.0009 & 0.0015 \\
\hline Mercury total (Hg), ng/L & 100 & 126 & 62.0 & 23.0 & 31.0 \\
\hline Methylmercury $\left(\mathrm{CH}_{3} \mathrm{Hg}\right), \mathrm{ng} / \mathrm{L}$ & 0.49 & --- & --- & --- & --- \\
\hline Nickel (Ni) & $<0.0005$ & $<0.0005$ & $<0.0005$ & $<0.0005$ & $<0.0005$ \\
\hline Lead $(\mathrm{Pb})$ & $<0.0008$ & $<0.0008$ & $<0.0008$ & $<0.0008$ & $<0.0008$ \\
\hline Beryllium (Be) & 0.002 & 0.001 & 0.001 & 0.001 & 0.001 \\
\hline Vanadium (V) & $<0.005$ & $<0.005$ & $<0.005$ & $<0.005$ & $<0.005$ \\
\hline Molybdenum (Mo) & 0.056 & $<0.007$ & $<0.007$ & $<0.007$ & $<0.007$ \\
\hline Antimony (Sb) & 0.039 & $<0.001$ & $<0.001$ & $<0.001$ & $<0.001$ \\
\hline Selenium (Se) & $<0.001$ & $<0.001$ & $<0.001$ & $<0.001$ & $<0.001$ \\
\hline Arsenic (As) & 2.35 & 2.11 & 2.15 & 2.11 & 1.98 \\
\hline Arsenite (As(III)) & 2.32 & $0.896^{2}$ & $1.56^{2}$ & $0.416^{2}$ & 0.131 \\
\hline Dissolved organic carbon (DOC) & 1.4 & 0.6 & 0.5 & 0.6 & 0.6 \\
\hline$\delta \mathrm{D}$, per mil & -134.59 & -146.42 & -147.00 & -145.95 & -144.69 \\
\hline$\delta^{18} 0$, per mil & -12.98 & -16.76 & -16.53 & -16.28 & -15.86 \\
\hline sum cations, meq/L & 18.2 & 13.9 & 14.2 & 14.5 & 15.0 \\
\hline sum anions, meq/L & 17.5 & 13.9 & 14.1 & 14.3 & 14.8 \\
\hline Charge imbalance, percent & 4.1 & 0.2 & 0.9 & 1.2 & 1.6 \\
\hline
\end{tabular}


Table 6. Results of water analyses for Norris Geyser Basin, 2006-2008-Continued.

\begin{tabular}{|c|c|c|c|c|c|c|}
\hline \multirow[b]{2}{*}{ Location } & \multicolumn{6}{|c|}{ 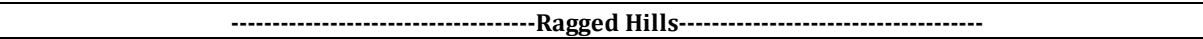 } \\
\hline & $\begin{array}{c}\text { "Succession } \\
\text { Spring" drainage } \\
\text { channel }\end{array}$ & $\begin{array}{l}\text { "Succession } \\
\text { Spring" Mat }\end{array}$ & "Titanic Spring" & "Titanic Spring" & $\begin{array}{l}\text { Unnamed pool } \\
\text { near the Gap }\end{array}$ & $\begin{array}{c}\text { Unnamed pool } \\
\text { near "Succession } \\
\text { Spring" }\end{array}$ \\
\hline Sample ID & 06WA130 & 06WA131 & 06WA117 & 07WA114 & 08WA121 & 06WA125 \\
\hline Collection Date / Time & $5 / 17 / 0612: 45$ & $5 / 17 / 060: 00$ & $5 / 15 / 0611: 40$ & 9/13/07 11:00 & $9 / 23 / 0814: 20$ & $5 / 17 / 069: 35$ \\
\hline Temperature, ${ }^{\circ} \mathrm{C}$ & 36.7 & 25.4 & 68.0 & 62.8 & 91.2 & 84.0 \\
\hline pH (field / laboratory) & $2.78 / 2.99$ & $2.72 / 2.98$ & $3.56 / 3.44$ & $3.10 / 3.00$ & $3.40 / 3.24$ & $4.70 / 4.72$ \\
\hline $\begin{array}{l}\text { Specific conductance } \\
\text { (field / laboratory), } \mu \mathrm{S} / \mathrm{cm}\end{array}$ & $2050 / 2150$ & $2610 / 2780$ & $2100 / 2250$ & $2160 / 2310$ & $1900 / 2170$ & $2380 / 2560$ \\
\hline Eh, V & -- & -- & 0.321 & 0.151 & 0.190 & 0.242 \\
\hline Density, $\mathrm{g} / \mathrm{mL}$ at $20^{\circ} \mathrm{C}$ & 0.9990 & --- & 0.9994 & 0.9993 & 0.9996 & 0.9994 \\
\hline Dissolved oxygen (DO), mg/L & --- & --- & --- & --- & --- & 1.1 \\
\hline \multicolumn{7}{|l|}{ Constituent, $\mathrm{mg} / \mathrm{L}^{1}$} \\
\hline Calcium (Ca) & 4.62 & 5.47 & 5.55 & 6.04 & 4.73 & 6.97 \\
\hline Magnesium (Mg) & 0.297 & 0.284 & 0.147 & 0.195 & 0.068 & 0.021 \\
\hline Sodium (Na) & 271 & 400 & 358 & 348 & 312 & 447 \\
\hline Potassium (K) & 29.7 & 50.4 & 48.5 & 43.3 & 38.5 & 34.8 \\
\hline Strontium (Sr) & 0.014 & 0.022 & 0.017 & 0.018 & 0.016 & 0.023 \\
\hline Barium (Ba) & 0.101 & 0.087 & 0.058 & 0.073 & 0.141 & 0.070 \\
\hline Lithium (Li) & 2.74 & 3.98 & 4.32 & 4.09 & 4.25 & 5.14 \\
\hline Sulfate $\left(\mathrm{SO}_{4}\right)$ & 117 & 135 & 89.5 & 176 & 78.9 & 27.2 \\
\hline Thiosulfate $\left(\mathrm{S}_{2} \mathrm{O}_{3}\right)$ & $<0.1$ & $<0.1$ & $<0.1$ & $<0.1$ & $<0.1$ & $<0.1$ \\
\hline Polythionate $\left(\mathrm{S}_{\mathrm{n}} \mathrm{O}_{6}\right), \mathrm{mM}$ & $<0.001$ & $<0.001$ & $<0.001$ & $<0.001$ & $<0.001$ & $<0.001$ \\
\hline Hydrogen sulfide $\left(\mathrm{H}_{2} \mathrm{~S}\right)$ & --- & --- & --- & 0.016 & 0.017 & 0.002 \\
\hline Alkalinity $\left(\mathrm{HCO}_{3}\right)$ & --- & -- & --- & -- & -- & $<1$ \\
\hline Acidity (free / total), mM & $1.18 / 1.81$ & --- & $0.46 / 0.78$ & $1.21 / 1.62$ & $0.53 / 1.14$ & -- \\
\hline Fluoride $(\mathrm{F})$ & 2.89 & 3.43 & 4.85 & 4.44 & 4.79 & 6.71 \\
\hline Chloride $(\mathrm{Cl})$ & 439 & 631 & 556 & 523 & 543 & 713 \\
\hline Bromide (Br) & 1.54 & 1.89 & 1.86 & 1.44 & 1.80 & 2.49 \\
\hline Nitrate $\left(\mathrm{NO}_{3}\right)$ & $<0.05$ & $<0.05$ & $<0.05$ & 0.08 & 0.11 & 0.06 \\
\hline Ammonium $\left(\mathrm{NH}_{4}\right)$ & 0.669 & 0.716 & 1.10 & 2.29 & 2.20 & 0.746 \\
\hline Silica $\left(\mathrm{SiO}_{2}\right)$ & 246 & 280 & 513 & 432 & 265 & 400 \\
\hline Boron (B) & 6.32 & 9.54 & 7.88 & 7.72 & 7.87 & 10.3 \\
\hline Aluminum (Al) & 3.77 & 4.04 & 1.05 & 1.91 & 3.08 & 0.14 \\
\hline Iron total $(\mathrm{Fe}(\mathrm{T}))$ & 0.941 & 1.83 & 1.05 & 4.58 & 0.810 & 0.011 \\
\hline Ferrous iron $(\mathrm{Fe}(\mathrm{II}))$ & 0.424 & 1.81 & 0.607 & 4.16 & 0.810 & 0.009 \\
\hline Manganese (Mn) & 0.042 & 0.039 & 0.034 & 0.046 & 0.017 & 0.004 \\
\hline Copper $(\mathrm{Cu})$ & $<0.0005$ & $<0.0005$ & $<0.0005$ & $<0.0005$ & $<0.0005$ & $<0.0005$ \\
\hline Zinc (Zn) & 0.009 & 0.113 & 0.014 & 0.027 & $<0.004$ & $<0.004$ \\
\hline Cadmium (Cd) & $<0.0001$ & $<0.0001$ & $<0.0001$ & $<0.0001$ & $<0.0001$ & $<0.0001$ \\
\hline Chromium (Cr) & $<0.0005$ & 0.0034 & 0.0005 & $<0.0005$ & 0.0009 & $<0.0005$ \\
\hline Cobalt (Co) & 0.0008 & 0.0032 & 0.0033 & $<0.0007$ & $<0.0007$ & 0.0013 \\
\hline Mercury total (Hg), ng/L & 63.0 & 71.0 & 30.0 & 38.0 & 100 & 41.0 \\
\hline Methylmercury $\left(\mathrm{CH}_{3} \mathrm{Hg}\right), \mathrm{ng} / \mathrm{L}$ & --- & 1.61 & --- & 0.10 & 0.39 & -- \\
\hline Nickel (Ni) & $<0.0005$ & $<0.0005$ & $<0.0005$ & $<0.0005$ & $<0.0005$ & $<0.0005$ \\
\hline Lead $(\mathrm{Pb})$ & $<0.0008$ & 0.0011 & $<0.0008$ & $<0.0008$ & $<0.0008$ & $<0.0008$ \\
\hline Beryllium (Be) & 0.001 & 0.002 & 0.002 & 0.002 & $<0.001$ & $<0.001$ \\
\hline Vanadium (V) & $<0.005$ & $<0.005$ & $<0.005$ & $<0.005$ & $<0.005$ & $<0.005$ \\
\hline Molybdenum (Mo) & $<0.007$ & $<0.007$ & 0.112 & 0.054 & 0.008 & 0.200 \\
\hline Antimony (Sb) & $<0.001$ & $<0.001$ & 0.101 & 0.021 & 0.164 & 0.168 \\
\hline Selenium (Se) & $<0.001$ & $<0.001$ & $<0.001$ & $<0.001$ & $<0.001$ & $<0.001$ \\
\hline Arsenic (As) & 2.08 & 2.88 & 1.62 & 2.61 & 3.10 & 3.06 \\
\hline Arsenite (As(III)) & 0.076 & $0.521^{2}$ & 0.083 & 0.403 & 2.87 & 0.702 \\
\hline Dissolved organic carbon (DOC) & 1.0 & 15.1 & 0.5 & 0.7 & 0.8 & 0.7 \\
\hline$\delta \mathrm{D}$, per mil & -143.22 & -128.86 & -136.27 & -129.61 & -132.13 & -141.89 \\
\hline$\delta^{18} 0$, per mil & -15.72 & -12.36 & -13.62 & -12.23 & -12.34 & -14.54 \\
\hline sum cations, meq/L & 15.3 & 22.0 & 18.1 & 18.3 & 16.2 & 21.5 \\
\hline sum anions, meq/L & 14.6 & 20.4 & 17.6 & 18.2 & 16.9 & 21.1 \\
\hline Charge imbalance, percent & 4.7 & 7.6 & 2.9 & 0.8 & -4.3 & 2.0 \\
\hline
\end{tabular}


Table 7. Results of water analyses for Gibbon Canyon and Geyser Springs Group, 2006-2008.

[ $\delta \mathrm{D}$ and $\delta 18 \mathrm{O}$, isotopic composition of hydrogen and oxygen relative to VSMOW; $\mathrm{m}$, meters; meq/L, milliequivalents per liter; $\mathrm{mg} / \mathrm{L}$, milligrams per liter; $\mathrm{mM}$, millimolar; $\mu \mathrm{S} / \mathrm{cm}$, microsiemens per centimeter; $\mathrm{ng} / \mathrm{L}$ nanograms per liter; $\mathrm{V}$, volts; ---, not measured; ${ }^{\circ} \mathrm{C}$, degrees Celsius; $<$, less than]

\begin{tabular}{|c|c|c|c|c|c|}
\hline & \multicolumn{2}{|c|}{--------Gibbon Canyon-------- } & \multicolumn{3}{|c|}{ 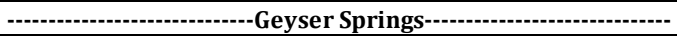 } \\
\hline Location & Beryl Spring & Beryl Spring & "Bullseye Spring" & $\begin{array}{c}\text { Unnamed pool } \\
\text { upslope from } \\
\text { "Bullseye Spring" }\end{array}$ & $\begin{array}{c}\text { Unnamed acid pool } \\
\text { near "Bullseye } \\
\text { Spring" }\end{array}$ \\
\hline Sample ID & 06WA135 & 06WA157 & 07WA108 & 08WA122 & 08WA117 \\
\hline Collection Date / Time & $5 / 17 / 0619: 45$ & $9 / 14 / 0616: 45$ & $9 / 10 / 07$ 17:10 & $9 / 20 / 0812: 00$ & $9 / 20 / 0812: 45$ \\
\hline Temperature, ${ }^{\circ} \mathrm{C}$ & 90.7 & 91.2 & 74.8 & 72.9 & 67.9 \\
\hline pH (field / laboratory) & $6.71 / 8.22$ & $6.67 / 7.69$ & $2.19 / 2.35$ & $2.40 / 2.27$ & $2.68 / 2.53$ \\
\hline $\begin{array}{l}\text { Specific conductance } \\
\text { (field / laboratory), } \mu \mathrm{S} / \mathrm{cm}\end{array}$ & $2040 / 2080$ & $2040 / 2190$ & 1868 / 2950 & $1940 / 3160$ & $1479 / 2230$ \\
\hline Eh, V & -0.030 & -0.164 & 0.087 & --- & --- \\
\hline Density, $\mathrm{g} / \mathrm{mL}$ at $20^{\circ} \mathrm{C}$ & 0.9993 & 0.9993 & 0.9990 & 0.9990 & 0.9992 \\
\hline Dissolved oxygen (DO), mg/L & --- & --- & --- & -- & --- \\
\hline \multicolumn{6}{|l|}{ Constituent, $\mathrm{mg} / \mathrm{L}^{1}$} \\
\hline Calcium (Ca) & 3.67 & 3.74 & 2.55 & 2.20 & 3.25 \\
\hline Magnesium (Mg) & 0.012 & 0.008 & 0.125 & 0.151 & 0.426 \\
\hline Sodium (Na) & 409 & 410 & 148 & 136 & 111 \\
\hline Potassium $(\mathrm{K})$ & 17.5 & 19.8 & 10.4 & 11.3 & 22.8 \\
\hline Strontium (Sr) & 0.007 & 0.007 & 0.002 & 0.002 & 0.011 \\
\hline Barium (Ba) & 0.001 & 0.001 & 0.001 & $<0.0008$ & 0.028 \\
\hline Lithium (Li) & 4.82 & 5.52 & 1.57 & 1.36 & 1.12 \\
\hline Sulfate $\left(\mathrm{SO}_{4}\right)$ & 62.2 & 67.8 & 714 & 552 & 673 \\
\hline Thiosulfate $\left(\mathrm{S}_{2} \mathrm{O}_{3}\right)$ & $<0.1$ & 0.2 & $<0.1$ & $<0.1$ & $--\cdot$ \\
\hline Polythionate $\left(\mathrm{S}_{\mathrm{n}} \mathrm{O}_{6}\right), \mathrm{mM}$ & $<0.001$ & 0.001 & $<0.001$ & $<0.001$ & --- \\
\hline Hydrogen sulfide $\left(\mathrm{H}_{2} \mathrm{~S}\right)$ & 0.39 & 0.42 & 0.07 & --- & --- \\
\hline Alkalinity $\left(\mathrm{HCO}_{3}\right)$ & 97.0 & 111 & --- & --- & --- \\
\hline Acidity (free / total), mM & --- & --- & $6.42 / 7.05$ & $6.01 / 6.96$ & $3.45 / 7.47$ \\
\hline Fluoride (F) & 17.1 & 18.4 & 3.51 & 2.78 & 0.589 \\
\hline Chloride (Cl) & 523 & 537 & 130 & 115 & 30.2 \\
\hline Bromide (Br) & 1.79 & 1.66 & 0.41 & 0.32 & 0.09 \\
\hline Nitrate $\left(\mathrm{NO}_{3}\right)$ & 0.18 & $<0.05$ & $<0.05$ & 0.19 & 0.09 \\
\hline Ammonium $\left(\mathrm{NH}_{4}\right)$ & 0.697 & 0.08 & 4.25 & 4.01 & --- \\
\hline Silica $\left(\mathrm{SiO}_{2}\right)$ & 299 & 284 & 221 & 217 & 172 \\
\hline Boron (B) & 7.14 & 7.41 & 1.87 & 1.55 & 0.499 \\
\hline Aluminum (Al) & 0.16 & 0.19 & 5.00 & 5.05 & 38.0 \\
\hline Iron total $(\mathrm{Fe}(\mathrm{T}))$ & $<0.002$ & 0.006 & 0.504 & 0.478 & 2.71 \\
\hline Ferrous iron $(\mathrm{Fe}(\mathrm{II}))$ & $<0.002$ & 0.006 & 0.112 & 0.092 & 2.58 \\
\hline Manganese (Mn) & 0.015 & 0.016 & 0.029 & 0.029 & 0.066 \\
\hline Copper $(\mathrm{Cu})$ & $<0.0005$ & 0.0010 & 0.0007 & $<0.0005$ & $<0.0005$ \\
\hline Zinc (Zn) & $<0.004$ & $<0.004$ & 0.016 & 0.011 & 0.135 \\
\hline Cadmium (Cd) & $<0.0001$ & $<0.0001$ & $<0.0001$ & $<0.0001$ & $<0.0001$ \\
\hline Chromium (Cr) & $<0.0005$ & $<0.0005$ & $<0.0005$ & $<0.0005$ & 0.0029 \\
\hline Cobalt (Co) & $<0.0007$ & $<0.0007$ & $<0.0007$ & $<0.0007$ & $<0.0007$ \\
\hline Mercury total (Hg), ng/L & 88.0 & 71.0 & 130 & 28.3 & --- \\
\hline Methylmercury $\left(\mathrm{CH}_{3} \mathrm{Hg}\right), \mathrm{ng} / \mathrm{L}$ & --- & 0.06 & 0.06 & 0.13 & --- \\
\hline Nickel (Ni) & $<0.0005$ & 0.0017 & $<0.0005$ & $<0.0005$ & 0.0080 \\
\hline Lead $(\mathrm{Pb})$ & $<0.0008$ & $<0.0008$ & $<0.0008$ & $<0.0008$ & 0.0080 \\
\hline Beryllium (Be) & 0.001 & 0.001 & 0.001 & 0.001 & 0.003 \\
\hline Vanadium (V) & $<0.005$ & $<0.005$ & $<0.005$ & $<0.005$ & $<0.005$ \\
\hline Molybdenum (Mo) & 0.131 & 0.136 & 0.014 & 0.017 & $<0.007$ \\
\hline Antimony (Sb) & 0.080 & 0.095 & 0.032 & 0.081 & 0.003 \\
\hline Selenium (Se) & $<0.001$ & 0.004 & $<0.001$ & $<0.001$ & $<0.001$ \\
\hline Arsenic (As) & 2.90 & 2.79 & 0.637 & 0.501 & 0.086 \\
\hline Arsenite (As(III)) & $2.45^{2}$ & $2.49^{2}$ & 0.099 & 0.033 & 0.062 \\
\hline Dissolved organic carbon (DOC) & 0.8 & 0.8 & 0.8 & 0.6 & --- \\
\hline$\delta \mathrm{D}$, per mil & -138.05 & -137.33 & -126.43 & -128.22 & --- \\
\hline$\delta^{18} 0$, per mil & -14.52 & -14.57 & -11.31 & -11.95 & $--\cdot$ \\
\hline sum cations, meq/L & 19.1 & 19.3 & 14.8 & 11.4 & 9.9 \\
\hline sum anions, meq/L & 18.6 & 19.3 & 14.0 & 11.9 & 10.7 \\
\hline Charge imbalance, percent & 2.9 & -0.2 & 5.7 & -4.1 & -8.0 \\
\hline
\end{tabular}


Table 8. Results of water analyses for the Crater Hills area, 2006-2008.

[ $\delta \mathrm{D}$ and $\delta 18 \mathrm{O}$, isotopic composition of hydrogen and oxygen relative to VSMOW; m, meters; meq/L, milliequivalents per liter; $\mathrm{mg} / \mathrm{L}$, milligrams per liter; $\mathrm{mM}$, millimolar; $\mu \mathrm{S} / \mathrm{cm}$, microsiemens per centimeter; $\mathrm{ng} / \mathrm{L}$ nanograms per liter; V, volts; ---, not measured; ${ }^{\circ} \mathrm{C}$, degrees Celsius; <, less than]

\begin{tabular}{|c|c|c|c|c|}
\hline \multirow[b]{2}{*}{ Location } & \multicolumn{4}{|c|}{ 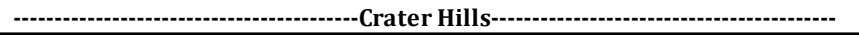 } \\
\hline & Sulphur Spring & Sulphur Spring & $\begin{array}{l}\text { Unnamed pool near } \\
\text { Sulphur Spring }\end{array}$ & $\begin{array}{c}\text { Unnamed acid pool } \\
\text { near Sulphur } \\
\text { Spring }\end{array}$ \\
\hline Sample ID & 07WA111 & 08WA115 & 07WA112 & 08WA116 \\
\hline Collection Date / Time & $9 / 11 / 0715: 15$ & $9 / 19 / 0813: 30$ & $9 / 11 / 0716: 30$ & 9/19/08 15:00 \\
\hline Temperature, ${ }^{\circ} \mathrm{C}$ & 87.8 & 87.2 & 82.0 & 73.8 \\
\hline pH (field / laboratory) & $3.86 / 3.83$ & $3.75 / 3.46$ & $2.27 / 2.31$ & $2.72 / 2.62$ \\
\hline \multicolumn{5}{|l|}{ Specific conductance } \\
\hline Eh, V & -0.029 & 0.110 & 0.179 & 0.210 \\
\hline Density, $\mathrm{g} / \mathrm{mL}$ at $20^{\circ} \mathrm{C}$ & 1.0004 & 1.0003 & 0.9988 & 1.0002 \\
\hline Dissolved oxygen (DO), mg/L & $--\cdot$ & --- & $--\cdot$ & --- \\
\hline \multicolumn{5}{|l|}{ Constituent, $\mathrm{mg} / \mathrm{L}^{1}$} \\
\hline Calcium (Ca) & 6.18 & 5.63 & 0.630 & 9.41 \\
\hline Magnesium (Mg) & 0.343 & 0.300 & 0.231 & 6.53 \\
\hline Sodium (Na) & 625 & 576 & 1.41 & 500 \\
\hline Potassium (K) & 106 & 117 & 1.38 & 94.3 \\
\hline Strontium (Sr) & 0.100 & 0.105 & 0.021 & 0.129 \\
\hline Barium (Ba) & 0.071 & 0.076 & 0.060 & 0.056 \\
\hline Lithium (Li) & 6.25 & 5.67 & 0.002 & 4.91 \\
\hline Sulfate $\left(\mathrm{SO}_{4}\right)$ & 454 & 425 & 787 & 650 \\
\hline Thiosulfate $\left(\mathrm{S}_{2} \mathrm{O}_{3}\right)$ & 1.6 & 1.4 & $<0.1$ & 0.2 \\
\hline Polythionate $\left(\mathrm{S}_{\mathrm{n}} \mathrm{O}_{6}\right), \mathrm{mM}$ & $<0.001$ & $<0.001$ & $<0.001$ & $<0.001$ \\
\hline Hydrogen sulfide $\left(\mathrm{H}_{2} \mathrm{~S}\right)$ & 1.21 & 1.03 & 0.16 & 0.73 \\
\hline Alkalinity $\left(\mathrm{HCO}_{3}\right)$ & --- & --- & --- & --- \\
\hline Acidity (free / total), mM & $0.81 / 1.79$ & $0.67 / 1.88$ & $8.50 / 8.77$ & $2.62 / 5.66$ \\
\hline Fluoride (F) & 38.1 & 37.3 & 0.039 & 18.7 \\
\hline Chloride (Cl) & 848 & 802 & 1.80 & 695 \\
\hline Bromide (Br) & 2.50 & 3.04 & 0.08 & 2.88 \\
\hline Nitrate $\left(\mathrm{NO}_{3}\right)$ & 0.07 & 0.34 & 0.42 & 0.05 \\
\hline Ammonium $\left(\mathrm{NH}_{4}\right)$ & 17.3 & 16.8 & 83.1 & 16.9 \\
\hline Silica $\left(\mathrm{SiO}_{2}\right)$ & 694 & 743 & 127 & 452 \\
\hline Boron (B) & 25.2 & 23.5 & 0.174 & 19.8 \\
\hline Aluminum (Al) & 6.15 & 5.92 & 2.97 & 17.9 \\
\hline Iron total $(\mathrm{Fe}(\mathrm{T}))$ & 0.154 & 0.141 & 0.588 & 0.704 \\
\hline Ferrous iron (Fe(II)) & 0.149 & 0.141 & 0.588 & 0.704 \\
\hline Manganese (Mn) & 0.234 & 0.226 & 0.010 & 0.634 \\
\hline Copper $(\mathrm{Cu})$ & $<0.0005$ & $<0.0005$ & 0.0008 & $<0.0005$ \\
\hline Zinc (Zn) & 0.009 & 0.004 & 0.026 & 0.039 \\
\hline Cadmium (Cd) & $<0.0001$ & $<0.0001$ & $<0.0001$ & $<0.0001$ \\
\hline Chromium (Cr) & 0.0011 & 0.0007 & 0.0031 & 0.0020 \\
\hline Cobalt (Co) & $<0.0007$ & $<0.0007$ & $<0.0007$ & $<0.0007$ \\
\hline Mercury total (Hg), ng/L & 7.6 & 6.4 & 380 & 35.8 \\
\hline Methylmercury $\left(\mathrm{CH}_{3} \mathrm{Hg}\right), \mathrm{ng} / \mathrm{L}$ & 0.18 & 0.42 & 1.09 & 0.12 \\
\hline Nickel (Ni) & $<0.0005$ & $<0.0005$ & 0.0045 & $<0.0005$ \\
\hline Lead $(\mathrm{Pb})$ & $<0.0008$ & 0.0050 & 0.0059 & 0.0080 \\
\hline Beryllium (Be) & 0.014 & 0.013 & $<0.001$ & 0.014 \\
\hline Vanadium (V) & $<0.005$ & $<0.005$ & $<0.005$ & 0.007 \\
\hline Molybdenum (Mo) & $<0.007$ & $<0.007$ & $<0.007$ & $<0.007$ \\
\hline Antimony (Sb) & 0.150 & 0.090 & 0.001 & 0.002 \\
\hline Selenium (Se) & $<0.001$ & $<0.001$ & $<0.001$ & $<0.001$ \\
\hline Arsenic (As) & 5.87 & 5.57 & 0.032 & 4.45 \\
\hline Arsenite (As(III)) & $5.61^{2}$ & $4.87^{2}$ & $<0.0005$ & $3.86^{2}$ \\
\hline Dissolved organic carbon (DOC) & 1.0 & 1.0 & 6.0 & 1.0 \\
\hline$\delta \mathrm{D}$, per mil & -124.94 & -126.58 & -109.32 & -116.88 \\
\hline$\delta^{18} 0$, per mil & -12.31 & -12.38 & -6.83 & -8.04 \\
\hline sum cations, meq/L & 31.8 & 30.0 & 10.9 & 29.4 \\
\hline sum anions, meq/L & 33.6 & 31.7 & 11.3 & 30.7 \\
\hline Charge imbalance, percent & -5.5 & -5.3 & -4.2 & -4.4 \\
\hline
\end{tabular}


Table 9. Results of water analyses for Ojo Caliente Spring and its discharge channel, Lower Geyser Basin, 20062008.

[ $\delta \mathrm{D}$ and $\delta 18 \mathrm{O}$, isotopic composition of hydrogen and oxygen relative to VSMOW; $\mathrm{m}$, meters; meq/L, milliequivalents per liter; $\mathrm{mg} / \mathrm{L}$, milligrams per liter; $\mathrm{mM}$, millimolar; $\mu \mathrm{S} / \mathrm{cm}$, microsiemens per centimeter; ng/L nanograms per liter; $\mathrm{V}$, volts; ---, not measured; ${ }^{\circ} \mathrm{C}$, degrees Celsius; $<$, less than]

\begin{tabular}{|c|c|c|c|c|c|}
\hline Location & $\begin{array}{c}\text { Ojo Caliente Spring } \\
\text { at discharge }\end{array}$ & $\begin{array}{l}\text { Ojo Caliente Spring } \\
\text { at pool exit }\end{array}$ & $\begin{array}{l}\text { Ojo Caliente Spring } \\
7.5 \text { m downstream } \\
\text { from pool exit }\end{array}$ & $\begin{array}{l}\text { Ojo Caliente Spring } \\
20.6 \mathrm{~m} \text { downstream } \\
\text { from pool exit }\end{array}$ & $\begin{array}{l}\text { Ojo Caliente Spring } \\
\text { at midpoint of pool }\end{array}$ \\
\hline Sample ID & 06WA160 & 06WA161 & 06WA162 & 06WA163 & 08WA113 \\
\hline Collection Date / Time & $9 / 16 / 069: 52$ & $9 / 16 / 069: 55$ & $9 / 16 / 069: 59$ & $9 / 16 / 0615: 10$ & $9 / 18 / 0817: 20$ \\
\hline Temperature, ${ }^{\circ} \mathrm{C}$ & 93.2 & 91.4 & 86.8 & 74.8 & 93.2 \\
\hline pH (field / laboratory) & $7.44 / 8.32$ & $7.52 / 8.33$ & $7.61 / 8.46$ & $---/ 8.63$ & $7.61 / 8.41$ \\
\hline $\begin{array}{l}\text { Specific conductance } \\
\text { (field / laboratory), } \mu \mathrm{S} / \mathrm{cm}\end{array}$ & $1470 / 1577$ & $1495 / 1578$ & $1530 / 1587$ & $1590 / 1618$ & $1470 / 1562$ \\
\hline Eh, V & -0.125 & --- & --- & --- & --- \\
\hline Density, $\mathrm{g} / \mathrm{mL}$ at $20^{\circ} \mathrm{C}$ & 0.9991 & 0.9991 & 0.9992 & 0.9992 & 0.9990 \\
\hline Dissolved oxygen (DO), mg/L & --- & --- & --- & --- & --- \\
\hline \multicolumn{6}{|l|}{ Constituent, mg/L ${ }^{1}$} \\
\hline Calcium (Ca) & 0.848 & 0.867 & 0.834 & 0.850 & 0.792 \\
\hline Magnesium (Mg) & $<0.001$ & $<0.001$ & $<0.001$ & $<0.001$ & $<0.001$ \\
\hline Sodium (Na) & 318 & 323 & 326 & 336 & 317 \\
\hline Potassium (K) & 9.30 & 9.18 & 9.41 & 9.32 & 8.91 \\
\hline Strontium (Sr) & 0.006 & 0.006 & 0.006 & 0.006 & 0.006 \\
\hline Barium (Ba) & $<0.0008$ & $<0.0008$ & $<0.0008$ & $<0.0008$ & $<0.0008$ \\
\hline Lithium (Li) & 3.52 & 3.58 & 3.61 & 3.72 & 3.41 \\
\hline Sulfate $\left(\mathrm{SO}_{4}\right)$ & 21.9 & 21.6 & 21.4 & 22.7 & 23.2 \\
\hline Thiosulfate $\left(\mathrm{S}_{2} \mathrm{O}_{3}\right)$ & 0.2 & 0.1 & 0.2 & 0.2 & 0.4 \\
\hline Polythionate $\left(\mathrm{S}_{\mathrm{n}} \mathrm{O}_{6}\right), \mathrm{mM}$ & 0.001 & $<0.001$ & $<0.001$ & $<0.001$ & -- \\
\hline Hydrogen sulfide $\left(\mathrm{H}_{2} \mathrm{~S}\right)$ & 0.86 & 0.96 & 0.59 & 0.12 & 0.86 \\
\hline Alkalinity $\left(\mathrm{HCO}_{3}\right)$ & 231 & 232 & 235 & 239 & 228 \\
\hline Acidity (free / total), mM & -- & -- & -- & -- & -- \\
\hline Fluoride $(\mathrm{F})$ & 30.7 & 34.5 & 34.5 & 31.4 & 35.2 \\
\hline Chloride (Cl) & 310 & 318 & 319 & 324 & 320 \\
\hline Bromide (Br) & 1.07 & 1.01 & 1.07 & 1.04 & 1.05 \\
\hline Nitrate $\left(\mathrm{NO}_{3}\right)$ & $<0.05$ & $<0.05$ & $<0.05$ & $<0.05$ & 0.15 \\
\hline Ammonium $\left(\mathrm{NH}_{4}\right)$ & $<0.07$ & $<0.07$ & $<0.07$ & $<0.07$ & 0.530 \\
\hline Silica $\left(\mathrm{SiO}_{2}\right)$ & 259 & 257 & 263 & 263 & 270 \\
\hline Boron (B) & 4.07 & 4.13 & 4.09 & 4.20 & 3.96 \\
\hline Aluminum (Al) & 0.25 & 0.26 & 0.25 & 0.26 & 0.26 \\
\hline Iron total $(\mathrm{Fe}(\mathrm{T}))$ & 0.003 & 0.007 & 0.008 & 0.002 & 0.004 \\
\hline Ferrous iron (Fe(II)) & 0.003 & 0.007 & 0.006 & 0.001 & 0.004 \\
\hline Manganese (Mn) & $<0.001$ & $<0.001$ & $<0.001$ & $<0.001$ & $<0.001$ \\
\hline Copper (Cu) & $<0.0005$ & $<0.0005$ & $<0.0005$ & $<0.0005$ & 0.0006 \\
\hline Zinc (Zn) & $<0.004$ & $<0.004$ & $<0.004$ & $<0.004$ & $<0.004$ \\
\hline Cadmium (Cd) & $<0.0001$ & $<0.0001$ & $<0.0001$ & $<0.0001$ & $<0.0001$ \\
\hline Chromium (Cr) & $<0.0005$ & $<0.0005$ & $<0.0005$ & $<0.0005$ & $<0.0005$ \\
\hline Cobalt (Co) & $<0.0007$ & $<0.0007$ & 0.0007 & $<0.0007$ & $<0.0007$ \\
\hline Mercury total (Hg), ng/L & 200 & 60.0 & 51.0 & 31.0 & 31.6 \\
\hline Methylmercury $\left(\mathrm{CH}_{3} \mathrm{Hg}\right), \mathrm{ng} / \mathrm{L}$ & 0.04 & 0.04 & 0.04 & 0.04 & --- \\
\hline Nickel (Ni) & $<0.0005$ & $<0.0005$ & $<0.0005$ & $<0.0005$ & $<0.0005$ \\
\hline Lead $(\mathrm{Pb})$ & $<0.0008$ & $<0.0008$ & $<0.0008$ & $<0.0008$ & $<0.0008$ \\
\hline Beryllium (Be) & 0.001 & 0.001 & 0.001 & 0.001 & $<0.001$ \\
\hline Vanadium (V) & $<0.005$ & $<0.005$ & $<0.005$ & $<0.005$ & $<0.005$ \\
\hline Molybdenum (Mo) & 0.036 & 0.038 & 0.040 & 0.042 & 0.034 \\
\hline Antimony (Sb) & 0.094 & 0.077 & 0.080 & 0.067 & 0.010 \\
\hline Selenium (Se) & $<0.001$ & $<0.001$ & $<0.001$ & 0.001 & $<0.001$ \\
\hline Arsenic (As) & 1.30 & 1.43 & 1.50 & 1.57 & 1.27 \\
\hline Arsenite (As(III)) & $1.02^{2}$ & $1.10^{2}$ & $1.24^{2}$ & $1.31^{2}$ & $0.831^{2}$ \\
\hline Dissolved organic carbon (DOC) & 0.9 & 0.5 & 0.5 & 0.6 & 0.7 \\
\hline$\delta \mathrm{D}$, per mil & -140.61 & -140.82 & -140.58 & -139.61 & -140.15 \\
\hline$\delta^{18} 0$, per mil & -16.49 & -16.52 & -16.43 & -16.18 & -16.51 \\
\hline sum cations, meq/L & 14.6 & 14.8 & 14.9 & 15.3 & 14.6 \\
\hline sum anions, meq/L & 14.6 & 15.0 & 15.1 & 15.0 & 15.1 \\
\hline Charge imbalance, percent & -0.3 & -1.4 & -0.9 & 1.6 & -3.5 \\
\hline
\end{tabular}


Table 9. Results of water analyses for Ojo Caliente Spring and its discharge channel, Lower Geyser Basin, 20062008-Continued.

\begin{tabular}{|c|c|c|c|c|c|}
\hline Location & $\begin{array}{l}\text { 0jo Caliente Spring } \\
\text { source, } 50 \text { feet } \\
\text { below surface }\end{array}$ & $\begin{array}{c}\text { Ojo Caliente Spring } \\
\text { at pool exit }\end{array}$ & $\begin{array}{c}\text { Ojo Caliente Spring } \\
13.8 \mathrm{~m} \text { downstream } \\
\text { from pool exit }\end{array}$ & $\begin{array}{l}\text { Ojo Caliente Spring } \\
20.4 \text { m downstream } \\
\text { from pool exit }\end{array}$ & $\begin{array}{l}\text { Ojo Caliente Spring } \\
25.9 \mathrm{~m} \text { downstream } \\
\text { from pool exit }\end{array}$ \\
\hline Sample ID & 08WA112 & 08WA111 & 08WA110 & 08WA109 & 08WA108 \\
\hline Collection Date / Time & 9/18/08 15:15 & 9/18/08 14:00 & $9 / 18 / 0812: 00$ & 9/18/08 11:00 & 9/18/08 10:20 \\
\hline Temperature, ${ }^{\circ} \mathrm{C}$ & --- & 91.9 & 84.2 & 75.4 & 67.5 \\
\hline pH (field / laboratory) & $7.60 / 8.44$ & $7.55 / 8.44$ & $7.70 / 8.44$ & $7.82 / 8.58$ & 8.17 / 8.68 \\
\hline $\begin{array}{l}\text { Specific conductance } \\
\text { (field / laboratory), } \mu \mathrm{S} / \mathrm{cm}\end{array}$ & $1523 / 1552$ & $1481 / 1570$ & $1517 / 1578$ & 1556 / 1601 & 1594 / 1624 \\
\hline Eh, V & -- & -- & --- & --- & 0.110 \\
\hline Density, $\mathrm{g} / \mathrm{mL}$ at $20^{\circ} \mathrm{C}$ & 0.9990 & 0.9990 & 0.9991 & 0.9990 & 0.9990 \\
\hline Dissolved oxygen (DO), mg/L & --- & --- & 0.1 & 1.7 & 2.6 \\
\hline \multicolumn{6}{|l|}{ Constituent, $\mathrm{mg} / \mathrm{L}^{1}$} \\
\hline Calcium (Ca) & 0.803 & 0.806 & 0.793 & 0.812 & 0.819 \\
\hline Magnesium (Mg) & $<0.001$ & $<0.001$ & $<0.001$ & $<0.001$ & $<0.001$ \\
\hline Sodium $(\mathrm{Na})$ & 311 & 336 & 328 & 327 & 336 \\
\hline Potassium (K) & 8.02 & 8.44 & 8.59 & 8.99 & 9.33 \\
\hline Strontium (Sr) & 0.006 & 0.006 & 0.006 & 0.006 & 0.006 \\
\hline Barium (Ba) & $<0.0008$ & $<0.0008$ & $<0.0008$ & $<0.0008$ & $<0.0008$ \\
\hline Lithium (Li) & 3.42 & 3.43 & 3.48 & 3.55 & 3.54 \\
\hline Sulfate $\left(\mathrm{SO}_{4}\right)$ & 22.3 & 21.8 & 21.8 & 22.4 & 23.4 \\
\hline Thiosulfate $\left(\mathrm{S}_{2} \mathrm{O}_{3}\right)$ & 0.4 & 0.5 & 0.7 & 0.8 & 0.4 \\
\hline Polythionate $\left(\mathrm{S}_{\mathrm{n}} \mathrm{O}_{6}\right), \mathrm{mM}$ & --- & --- & --- & --- & --- \\
\hline Hydrogen sulfide $\left(\mathrm{H}_{2} \mathrm{~S}\right)$ & 0.95 & 0.88 & 0.63 & 0.06 & 0.03 \\
\hline Alkalinity $\left(\mathrm{HCO}_{3}\right)$ & 226 & 229 & 230 & 233 & 236 \\
\hline Acidity (free / total), mM & --- & --- & --- & --- & --- \\
\hline Fluoride (F) & 35.1 & 35.4 & 35.8 & 36.5 & 37.0 \\
\hline Chloride (Cl) & 322 & 322 & 326 & 330 & 339 \\
\hline Bromide (Br) & 1.16 & 0.98 & 1.00 & 1.00 & 1.24 \\
\hline Nitrate $\left(\mathrm{NO}_{3}\right)$ & 0.17 & 0.17 & 0.18 & 0.18 & 0.25 \\
\hline Ammonium $\left(\mathrm{NH}_{4}\right)$ & 0.540 & 0.620 & 0.550 & 0.450 & 0.370 \\
\hline Silica $\left(\mathrm{SiO}_{2}\right)$ & 244 & 229 & 225 & 237 & 256 \\
\hline Boron (B) & 3.88 & 3.97 & 3.99 & 4.09 & 4.18 \\
\hline Aluminum (Al) & 0.27 & 0.28 & 0.26 & 0.27 & 0.27 \\
\hline Iron total $(\mathrm{Fe}(\mathrm{T}))$ & $<0.002$ & $<0.002$ & 0.005 & $<0.002$ & 0.005 \\
\hline Ferrous iron $(\mathrm{Fe}(\mathrm{II}))$ & $<0.002$ & $<0.002$ & 0.005 & $<0.002$ & 0.004 \\
\hline Manganese (Mn) & $<0.001$ & $<0.001$ & $<0.001$ & $<0.001$ & $<0.001$ \\
\hline Copper $(\mathrm{Cu})$ & $<0.0005$ & $<0.0005$ & $<0.0005$ & $<0.0005$ & $<0.0005$ \\
\hline Zinc (Zn) & $<0.004$ & $<0.004$ & $<0.004$ & $<0.004$ & $<0.004$ \\
\hline Cadmium (Cd) & $<0.0001$ & $<0.0001$ & $<0.0001$ & $<0.0001$ & $<0.0001$ \\
\hline Chromium (Cr) & $<0.0005$ & $<0.0005$ & $<0.0005$ & $<0.0005$ & $<0.0005$ \\
\hline Cobalt (Co) & $<0.0007$ & $<0.0007$ & $<0.0007$ & $<0.0007$ & $<0.0007$ \\
\hline Mercury total (Hg), ng/L & 30.0 & 33.4 & 35.6 & 27.0 & 20.2 \\
\hline Methylmercury $\left(\mathrm{CH}_{3} \mathrm{Hg}\right), \mathrm{ng} / \mathrm{L}$ & --- & --- & --- & --- & --- \\
\hline Nickel (Ni) & $<0.0005$ & $<0.0005$ & $<0.0005$ & $<0.0005$ & $<0.0005$ \\
\hline Lead $(\mathrm{Pb})$ & $<0.0008$ & $<0.0008$ & $<0.0008$ & $<0.0008$ & $<0.0008$ \\
\hline Beryllium (Be) & 0.001 & 0.001 & $<0.001$ & $<0.001$ & $<0.001$ \\
\hline Vanadium (V) & $<0.005$ & $<0.005$ & $<0.005$ & $<0.005$ & $<0.005$ \\
\hline Molybdenum (Mo) & 0.037 & 0.037 & 0.038 & 0.042 & 0.039 \\
\hline Antimony (Sb) & 0.026 & 0.037 & 0.063 & 0.065 & 0.068 \\
\hline Selenium (Se) & $<0.001$ & $<0.001$ & $<0.001$ & $<0.001$ & $<0.001$ \\
\hline Arsenic (As) & 1.34 & 1.37 & 1.40 & 1.43 & 1.47 \\
\hline Arsenite (As(III)) & $0.566^{2}$ & $0.866^{2}$ & $1.15^{2}$ & $1.04^{2}$ & $0.307^{2}$ \\
\hline Dissolved organic carbon (DOC) & 0.7 & 0.6 & 0.6 & 0.5 & 1.2 \\
\hline$\delta \mathrm{D}$, per mil & -141.01 & -140.03 & -138.72 & -137.48 & -137.47 \\
\hline$\delta^{18} 0$, per mil & -16.47 & -16.41 & -16.28 & -16.09 & -15.89 \\
\hline sum cations, meq/L & 14.3 & 15.3 & 15.0 & 15.0 & 15.4 \\
\hline sum anions, meq/L & 15.1 & 15.1 & 15.3 & 15.5 & 15.9 \\
\hline Charge imbalance, percent & -5.4 & 1.5 & -1.8 & -3.1 & -3.0 \\
\hline
\end{tabular}

\footnotetext{
${ }^{1}$ Except for acidity (mM), mercury (ng/L), methylmercury (ng/L), and $\delta \mathrm{D}$ and $\delta^{18} \mathrm{O}$ (per mil)
}

${ }^{2}$ Likely formed arsenic - sulfide precipitate upon acidification; therefore, value may be biased low 
Table 10. Results of water analyses for the Porcupine Hills area, 2006-2008.

[ $\delta \mathrm{D}$ and $\delta 18 \mathrm{O}$, isotopic composition of hydrogen and oxygen relative to VSMOW; m, meters; meq/L, milliequivalents per liter; $\mathrm{mg} / \mathrm{L}$, milligrams per liter; $\mathrm{mM}$, millimolar; $\mu \mathrm{S} / \mathrm{cm}$, microsiemens per centimeter; $\mathrm{ng} / \mathrm{L}$ nanograms per liter; V, volts; ---, not measured; ${ }^{\circ} \mathrm{C}$, degrees Celsius; $<$, less than]

\begin{tabular}{|c|c|c|c|c|}
\hline Location & Snort Geyser & $\begin{array}{l}\text { Unnamed hot spring, } \\
\text { Morning Mist Group }\end{array}$ & $\begin{array}{l}\text { Unnamed hot spring, } \\
\text { Quagmire Group }\end{array}$ & $\begin{array}{l}\text { Unnamed hot spring, } \\
\text { Quagmire Group }\end{array}$ \\
\hline Sample ID & 06WA173 & 06WA170 & 06WA171 & 06WA172 \\
\hline Collection Date / Time & $9 / 19 / 0614: 35$ & $9 / 19 / 0610: 45$ & 9/19/06 12:15 & $9 / 19 / 0613: 55$ \\
\hline Temperature, ${ }^{\circ} \mathrm{C}$ & 92.0 & 75.2 & 93.8 & 65.9 \\
\hline pH (field / laboratory) & $8.73 / 9.40$ & $9.17 / 9.61$ & $7.64 / 8.16$ & $6.68 / 7.89$ \\
\hline \multicolumn{5}{|l|}{ Specific conductance } \\
\hline (field / laboratory), $\mu \mathrm{S} / \mathrm{cm}$ & $1450 / 1542$ & 1557 / 1588 & 1310 / 1398 & $1638 / 1565$ \\
\hline Eh, V & -0.185 & -0.086 & -0.145 & 0.136 \\
\hline Density, $\mathrm{g} / \mathrm{mL}$ at $20^{\circ} \mathrm{C}$ & 0.9991 & 0.9991 & 0.9990 & 0.9991 \\
\hline Dissolved oxygen (DO), mg/L & --- & --- & --- & --- \\
\hline \multicolumn{5}{|l|}{ Constituent, mg/L ${ }^{1}$} \\
\hline Calcium (Ca) & 1.35 & 0.791 & 1.40 & 1.26 \\
\hline Magnesium (Mg) & $<0.001$ & $<0.001$ & $<0.001$ & $<0.001$ \\
\hline Sodium (Na) & 311 & 323 & 278 & 311 \\
\hline Potassium (K) & 10.6 & 8.55 & 8.43 & 9.76 \\
\hline Strontium (Sr) & 0.003 & 0.003 & 0.001 & 0.003 \\
\hline Barium (Ba) & $<0.0008$ & 0.001 & $<0.0008$ & 0.001 \\
\hline Lithium (Li) & 3.46 & 3.43 & 3.23 & 2.76 \\
\hline Sulfate $\left(\mathrm{SO}_{4}\right)$ & 17.9 & 19.3 & 19.3 & 55.0 \\
\hline Thiosulfate $\left(\mathrm{S}_{2} \mathrm{O}_{3}\right)$ & $<0.1$ & 0.3 & $<0.1$ & $<0.1$ \\
\hline Polythionate $\left(\mathrm{S}_{\mathrm{n}} \mathrm{O}_{6}\right), \mathrm{mM}$ & $<0.001$ & $<0.001$ & $<0.001$ & $<0.001$ \\
\hline Hydrogen sulfide $\left(\mathrm{H}_{2} \mathrm{~S}\right)$ & 0.83 & 0.03 & 0.28 & --- \\
\hline Alkalinity $\left(\mathrm{HCO}_{3}\right)$ & 170 & 252 & 133 & 144 \\
\hline Acidity (free / total), mM & --- & --- & --- & --- \\
\hline Fluoride (F) & 30.8 & 31.0 & 29.0 & 28.6 \\
\hline Chloride (Cl) & 335 & 302 & 310 & 330 \\
\hline Bromide (Br) & 1.13 & 0.96 & 0.99 & 1.03 \\
\hline Nitrate $\left(\mathrm{NO}_{3}\right)$ & $<0.05$ & $<0.05$ & $<0.05$ & $<0.05$ \\
\hline Ammonium $\left(\mathrm{NH}_{4}\right)$ & $<0.07$ & 0.170 & $<0.07$ & $<0.07$ \\
\hline Silica $\left(\mathrm{SiO}_{2}\right)$ & 206 & 218 & 240 & 239 \\
\hline Boron (B) & 4.46 & 4.10 & 4.07 & 4.43 \\
\hline Aluminum (Al) & 0.12 & 0.12 & 0.15 & 0.13 \\
\hline Iron total $(\mathrm{Fe}(\mathrm{T}))$ & 0.006 & 0.011 & 0.007 & 0.005 \\
\hline Ferrous iron (Fe(II)) & 0.006 & 0.009 & 0.006 & 0.001 \\
\hline Manganese (Mn) & $<0.001$ & $<0.001$ & $<0.001$ & 0.001 \\
\hline Copper $(\mathrm{Cu})$ & $<0.0005$ & 0.0020 & $<0.0005$ & $<0.0005$ \\
\hline Zinc (Zn) & $<0.004$ & $<0.004$ & $<0.004$ & 0.007 \\
\hline Cadmium (Cd) & $<0.0001$ & $<0.0001$ & $<0.0001$ & $<0.0001$ \\
\hline Chromium (Cr) & $<0.0005$ & $<0.0005$ & $<0.0005$ & $<0.0005$ \\
\hline Cobalt (Co) & $<0.0007$ & $<0.0007$ & $<0.0007$ & $<0.0007$ \\
\hline Mercury total $(\mathrm{Hg}), \mathrm{ng} / \mathrm{L}$ & 100 & 73.0 & 118 & 47.0 \\
\hline Methylmercury $\left(\mathrm{CH}_{3} \mathrm{Hg}\right), \mathrm{ng} / \mathrm{L}$ & 0.04 & 0.04 & 0.04 & 0.04 \\
\hline Nickel (Ni) & $<0.0005$ & $<0.0005$ & $<0.0005$ & $<0.0005$ \\
\hline Lead $(\mathrm{Pb})$ & $<0.0008$ & $<0.0008$ & $<0.0008$ & $<0.0008$ \\
\hline Beryllium (Be) & $<0.001$ & $<0.001$ & $<0.001$ & $<0.001$ \\
\hline Vanadium (V) & $<0.005$ & $<0.005$ & $<0.005$ & $<0.005$ \\
\hline Molybdenum (Mo) & 0.164 & 0.105 & 0.149 & 0.095 \\
\hline Antimony (Sb) & 0.093 & 0.062 & 0.081 & 0.123 \\
\hline Selenium (Se) & $<0.001$ & 0.001 & $<0.001$ & 0.002 \\
\hline Arsenic (As) & 1.69 & 1.35 & 1.56 & 2.08 \\
\hline Arsenite (As(III)) & $1.35^{2}$ & $0.402^{2}$ & $1.34^{2}$ & $0.069^{2}$ \\
\hline Dissolved organic carbon (DOC) & 0.6 & 0.8 & 0.6 & 0.8 \\
\hline$\delta \mathrm{D}$, per mil & -141.17 & -140.51 & -139.67 & -132.92 \\
\hline$\delta^{18} 0$, per mil & -16.52 & -16.18 & -16.21 & -13.13 \\
\hline sum cations, meq/L & 14.2 & 14.6 & 12.8 & 14.2 \\
\hline sum anions, meq/L & 14.2 & 14.5 & 12.9 & 14.3 \\
\hline Charge imbalance, percent & 0.4 & 0.4 & -0.4 & -0.7 \\
\hline
\end{tabular}


Table 10. Results of water analyses for the Porcupine Hills area, 2006-2008-Continued.

\begin{tabular}{|c|c|c|c|c|}
\hline Location & $\begin{array}{l}\text { Unnamed hot spring, } \\
\text { Quagmire Group }\end{array}$ & $\begin{array}{l}\text { Unnamed hot spring, } \\
\text { Quagmire Group }\end{array}$ & $\begin{array}{l}\text { Unnamed hot spring, } \\
\text { Quagmire Group }\end{array}$ & $\begin{array}{l}\text { Unnamed hot spring, } \\
\text { Quagmire Group }\end{array}$ \\
\hline Sample ID & 06WA174 & 08WA132 & 08WA133 & 08WA134 \\
\hline Collection Date / Time & $9 / 19 / 0615: 20$ & $9 / 24 / 0816: 15$ & $9 / 24 / 0817: 08$ & 9/24/08 18:00 \\
\hline Temperature, ${ }^{\circ} \mathrm{C}$ & 84.8 & 92.9 & 65.6 & 92.3 \\
\hline pH (field / laboratory) & $8.68 / 9.23$ & $7.56 / 8.30$ & $6.94 / 8.27$ & $8.59 / 9.02$ \\
\hline Specific conductance & & & & \\
\hline (field / laboratory), $\mu \mathrm{S} / \mathrm{cm}$ & $1400 / 1452$ & 1303 / 1371 & $1500 / 1525$ & $1340 / 1424$ \\
\hline Eh, V & -0.124 & 0.000 & --- & -0.050 \\
\hline Density, $\mathrm{g} / \mathrm{mL}$ at $20^{\circ} \mathrm{C}$ & 0.9990 & 0.9989 & 0.9990 & 0.9989 \\
\hline Dissolved oxygen (DO), mg/L & --- & --- & --- & --- \\
\hline \multicolumn{5}{|l|}{ Constituent, mg/ $\mathrm{L}^{1}$} \\
\hline Calcium (Ca) & 1.17 & 1.38 & 1.21 & 1.15 \\
\hline Magnesium (Mg) & $<0.001$ & 0.001 & 0.001 & 0.002 \\
\hline Sodium (Na) & 295 & 260 & 289 & 274 \\
\hline Potassium (K) & 8.40 & 6.49 & 8.21 & 7.57 \\
\hline Strontium (Sr) & 0.003 & 0.002 & 0.003 & 0.003 \\
\hline Barium (Ba) & $<0.0008$ & $<0.0008$ & $<0.0008$ & $<0.0008$ \\
\hline Lithium (Li) & 2.38 & 3.13 & 2.70 & 2.37 \\
\hline Sulfate $\left(\mathrm{SO}_{4}\right)$ & 20.6 & 20.8 & 55.3 & 22.0 \\
\hline Thiosulfate $\left(\mathrm{S}_{2} \mathrm{O}_{3}\right)$ & 0.2 & 0.6 & $<0.1$ & 0.2 \\
\hline Polythionate $\left(\mathrm{S}_{\mathrm{n}} \mathrm{O}_{6}\right), \mathrm{mM}$ & $<0.001$ & --- & --- & --- \\
\hline Hydrogen sulfide $\left(\mathrm{H}_{2} \mathrm{~S}\right)$ & 0.27 & 0.44 & 0.001 & 0.50 \\
\hline Alkalinity $\left(\mathrm{HCO}_{3}\right)$ & 196 & 128 & 143 & 187 \\
\hline Acidity (free / total), mM & --- & --- & --- & --- \\
\hline Fluoride (F) & 27.5 & 34.0 & 33.6 & 30.2 \\
\hline Chloride $(\mathrm{Cl})$ & 300 & 299 & 311 & 292 \\
\hline Bromide (Br) & 0.95 & 1.04 & 1.02 & 0.90 \\
\hline Nitrate $\left(\mathrm{NO}_{3}\right)$ & $<0.05$ & 0.21 & 0.24 & 0.23 \\
\hline Ammonium $\left(\mathrm{NH}_{4}\right)$ & $<0.07$ & 0.140 & 0.190 & 0.170 \\
\hline Silica $\left(\mathrm{SiO}_{2}\right)$ & 190 & 212 & 231 & 212 \\
\hline Boron (B) & 3.88 & 3.86 & 4.23 & 3.78 \\
\hline Aluminum (Al) & 0.16 & 0.15 & 0.18 & 0.17 \\
\hline Iron total $(\mathrm{Fe}(\mathrm{T}))$ & 0.009 & $<0.002$ & $<0.002$ & $<0.002$ \\
\hline Ferrous iron (Fe(II)) & 0.006 & $<0.002$ & $<0.002$ & $<0.002$ \\
\hline Manganese (Mn) & $<0.001$ & 0.002 & 0.002 & $<0.001$ \\
\hline Copper $(\mathrm{Cu})$ & $<0.0005$ & $<0.0005$ & $<0.0005$ & 0.0008 \\
\hline Zinc (Zn) & $<0.004$ & $<0.004$ & $<0.004$ & $<0.004$ \\
\hline Cadmium (Cd) & $<0.0001$ & $<0.0001$ & $<0.0001$ & $<0.0001$ \\
\hline Chromium (Cr) & $<0.0005$ & $<0.0005$ & $<0.0005$ & $<0.0005$ \\
\hline Cobalt (Co) & $<0.0007$ & $<0.0007$ & $<0.0007$ & $<0.0007$ \\
\hline Mercury total (Hg), ng/L & 57.0 & 74.4 & 42.1 & 31.3 \\
\hline Methylmercury $\left(\mathrm{CH}_{3} \mathrm{Hg}\right), \mathrm{ng} / \mathrm{L}$ & 0.04 & --- & --- & --- \\
\hline Nickel (Ni) & $<0.0005$ & $<0.0005$ & $<0.0005$ & $<0.0005$ \\
\hline Lead $(\mathrm{Pb})$ & $<0.0008$ & $<0.0008$ & $<0.0008$ & $<0.0008$ \\
\hline Beryllium (Be) & $<0.001$ & $<0.001$ & $<0.001$ & $<0.001$ \\
\hline Vanadium (V) & $<0.005$ & $<0.005$ & $<0.005$ & $<0.005$ \\
\hline Molybdenum (Mo) & 0.173 & 0.147 & 0.092 & 0.167 \\
\hline Antimony (Sb) & 0.085 & 0.098 & 0.121 & 0.096 \\
\hline Selenium (Se) & $<0.001$ & $<0.001$ & $<0.001$ & $<0.001$ \\
\hline Arsenic (As) & 1.56 & 1.50 & 1.95 & 1.52 \\
\hline Arsenite (As(III)) & $1.35^{2}$ & $1.28^{2}$ & $0.094^{2}$ & $1.16^{2}$ \\
\hline Dissolved organic carbon (DOC) & 0.7 & 1.1 & 0.8 & 1.3 \\
\hline$\delta \mathrm{D}$, per mil & -140.49 & -139.59 & -132.54 & -142.38 \\
\hline$\delta^{18} 0$, per mil & -16.14 & -16.22 & -13.01 & -16.25 \\
\hline sum cations, meq/L & 13.3 & 12.0 & 13.2 & 12.4 \\
\hline sum anions, meq/L & 13.4 & 12.8 & 14.0 & 13.3 \\
\hline Charge imbalance, percent & -1.2 & -6.4 & -6.0 & -7.0 \\
\hline
\end{tabular}


Table 11. Results of water analyses for Midway Geyser Basin and the Rabbit Creek area, 2006-2008.

[ $\delta \mathrm{D}$ and $\delta 18 \mathrm{O}$, isotopic composition of hydrogen and oxygen relative to VSMOW; m, meters; meq/L, milliequivalents per liter; $\mathrm{mg} / \mathrm{L}$, milligrams per liter; $\mathrm{mM}$, millimolar; $\mu \mathrm{S} / \mathrm{cm}$, microsiemens per centimeter; $\mathrm{ng} / \mathrm{L}$ nanograms per liter; V, volts; ---, not measured; ${ }^{\circ} \mathrm{C}$, degrees Celsius; <, less than]

\begin{tabular}{|c|c|c|c|c|c|}
\hline & -Midway Geyser Basin- & - & Rabbit Cr & eek area---.-... & - \\
\hline Location & Excelsior Geyser Crater & $\begin{array}{c}\text { Unnamed hot } \\
\text { spring }\end{array}$ & $\begin{array}{c}\text { Unnamed hot } \\
\text { spring drainage } \\
\text { channel }\end{array}$ & $\begin{array}{c}\text { Unnamed hot } \\
\text { spring drainage } \\
\text { channel }\end{array}$ & $\begin{array}{c}\text { Unnamed hot } \\
\text { spring drainage } \\
\text { channel }\end{array}$ \\
\hline Sample ID & 07WA148 & 06WA113 & 06WA114 & 06WA114B & 06WA114C \\
\hline Collection Date / Time & 9/19/07 10:30 & $5 / 14 / 0611: 15$ & $5 / 14 / 0613: 00$ & 5/14/06 13:00 & $5 / 14 / 0613: 00$ \\
\hline Temperature, ${ }^{\circ} \mathrm{C}$ & 88.7 & 85.8 & 84.0 & 69.5 & 61.8 \\
\hline pH (field / laboratory) & $7.68 / 8.59$ & $9.19 / 9.68$ & $9.21 / 9.77$ & $9.21 / 9.76$ & $9.29 / 9.80$ \\
\hline \multicolumn{6}{|l|}{ Specific conductance } \\
\hline $\mathrm{Eh}, \mathrm{V}$ & -0.165 & 0.068 & 0.046 & --- & --- \\
\hline Density, $\mathrm{g} / \mathrm{mL}$ at $20^{\circ} \mathrm{C}$ & 0.9993 & 0.9992 & 0.9991 & 0.9993 & 0.9992 \\
\hline Dissolved oxygen (DO), mg/L & --- & --- & --- & --- & --- \\
\hline \multicolumn{6}{|l|}{ Constituent, $\mathrm{mg} / \mathrm{L}^{1}$} \\
\hline Calcium (Ca) & 1.54 & 0.347 & 0.308 & 0.326 & 0.340 \\
\hline Magnesium (Mg) & 0.007 & 0.001 & 0.001 & $<0.001$ & $<0.001$ \\
\hline Sodium (Na) & 374 & 323 & 343 & 353 & 369 \\
\hline Potassium (K) & 12.3 & 10.5 & 10.3 & 10.7 & 11.1 \\
\hline Strontium (Sr) & 0.006 & 0.002 & 0.002 & 0.002 & 0.002 \\
\hline Barium (Ba) & 0.003 & $<0.0008$ & $<0.0008$ & $<0.0008$ & $<0.0008$ \\
\hline Lithium (Li) & 2.37 & 3.60 & 3.91 & 4.08 & 4.17 \\
\hline Sulfate $\left(\mathrm{SO}_{4}\right)$ & 15.0 & 21.6 & 19.5 & 20.2 & 21.0 \\
\hline Thiosulfate $\left(\mathrm{S}_{2} \mathrm{O}_{3}\right)$ & $<0.1$ & 0.7 & 0.8 & 0.8 & $<0.1$ \\
\hline Polythionate $\left(\mathrm{S}_{\mathrm{n}} \mathrm{O}_{6}\right), \mathrm{mM}$ & $<0.001$ & $<0.001$ & $<0.001$ & $<0.001$ & $<0.001$ \\
\hline Hydrogen sulfide $\left(\mathrm{H}_{2} \mathrm{~S}\right)$ & 0.011 & 0.009 & 0.005 & $<0.002$ & --- \\
\hline Alkalinity $\left(\mathrm{HCO}_{3}\right)$ & 503 & 339 & 349 & 363 & 377 \\
\hline Acidity (free / total), mM & --- & --- & -- & --- & -- \\
\hline Fluoride (F) & 26.0 & 26.2 & 27.0 & 28.6 & 29.8 \\
\hline Chloride $(\mathrm{Cl})$ & 258 & 260 & 266 & 278 & 284 \\
\hline Bromide (Br) & 0.84 & 1.98 & 1.06 & 1.12 & 1.13 \\
\hline Nitrate $\left(\mathrm{NO}_{3}\right)$ & 0.14 & 0.18 & 0.38 & 0.11 & $<0.05$ \\
\hline Ammonium $\left(\mathrm{NH}_{4}\right)$ & $<0.07$ & 0.317 & 0.415 & --- & --- \\
\hline Silica $\left(\mathrm{SiO}_{2}\right)$ & 288 & 319 & 300 & 263 & 264 \\
\hline Boron (B) & 3.01 & 2.73 & 2.95 & 3.03 & 3.13 \\
\hline Aluminum (Al) & 0.27 & 0.18 & 0.18 & 0.22 & 0.22 \\
\hline Iron total $(\mathrm{Fe}(\mathrm{T}))$ & $<0.002$ & $<0.002$ & $<0.002$ & $<0.002$ & $<0.002$ \\
\hline Ferrous iron (Fe(II)) & $<0.002$ & $<0.002$ & $<0.002$ & $<0.002$ & $<0.002$ \\
\hline Manganese (Mn) & 0.011 & $<0.001$ & $<0.001$ & $<0.001$ & $<0.001$ \\
\hline Copper (Cu) & $<0.0005$ & $<0.0005$ & $<0.0005$ & $<0.0005$ & $<0.0005$ \\
\hline Zinc (Zn) & $<0.004$ & $<0.004$ & $<0.004$ & $<0.004$ & $<0.004$ \\
\hline Cadmium (Cd) & $<0.0001$ & $<0.0001$ & $<0.0001$ & $<0.0001$ & $<0.0001$ \\
\hline Chromium (Cr) & $<0.0005$ & $<0.0005$ & $<0.0005$ & $<0.0005$ & $<0.0005$ \\
\hline Cobalt (Co) & $<0.0007$ & $<0.0007$ & $<0.0007$ & $<0.0007$ & $<0.0007$ \\
\hline Mercury total (Hg), ng/L & 48.0 & 45.0 & 45.0 & 35.0 & 16.0 \\
\hline Methylmercury $\left(\mathrm{CH}_{3} \mathrm{Hg}\right), \mathrm{ng} / \mathrm{L}$ & 0.08 & --- & --- & --- & --- \\
\hline Nickel (Ni) & $<0.0005$ & $<0.0005$ & $<0.0005$ & $<0.0005$ & $<0.0005$ \\
\hline Lead $(\mathrm{Pb})$ & $<0.0008$ & $<0.0008$ & $<0.0008$ & $<0.0008$ & $<0.0008$ \\
\hline Beryllium (Be) & 0.003 & $<0.001$ & $<0.001$ & $<0.001$ & $<0.001$ \\
\hline Vanadium (V) & $<0.005$ & $<0.005$ & $<0.005$ & $<0.005$ & $<0.005$ \\
\hline Molybdenum (Mo) & 0.031 & 0.030 & 0.029 & 0.030 & 0.019 \\
\hline Antimony (Sb) & 0.067 & 0.082 & 0.086 & 0.000 & 0.000 \\
\hline Selenium (Se) & $<0.001$ & $<0.001$ & $<0.001$ & $<0.001$ & $<0.001$ \\
\hline Arsenic (As) & 1.87 & 1.54 & 1.67 & 1.77 & 1.81 \\
\hline Arsenite (As(III)) & 0.064 & $0.225^{2}$ & $0.250^{2}$ & $0.194^{2}$ & $0.084^{2}$ \\
\hline Dissolved organic carbon (DOC) & 1.2 & 1.3 & 1.3 & --- & --- \\
\hline$\delta \mathrm{D}$, per mil & -141.12 & -141.24 & -141.37 & -141.13 & -138.87 \\
\hline$\delta^{18} 0$, per mil & -16.37 & -16.14 & -16.07 & -15.81 & -15.29 \\
\hline sum cations, meq/L & 16.9 & 14.5 & 15.4 & 15.8 & 16.6 \\
\hline sum anions, meq/L & 17.1 & 14.5 & 14.7 & 15.3 & 15.8 \\
\hline Charge imbalance, percent & -1.4 & 0.4 & 4.3 & 3.1 & 4.6 \\
\hline
\end{tabular}


Table 11. Results of water analyses for Midway Geyser Basin and the Rabbit Creek area, 2006-2008_Continued.

\begin{tabular}{|c|c|c|c|c|c|c|}
\hline & \multicolumn{6}{|c|}{ - - - } \\
\hline Location & $\begin{array}{c}\text { Unnamed hot } \\
\text { spring drainage } \\
\text { channel }\end{array}$ & $\begin{array}{l}\text { Unnamed hot } \\
\text { spring }\end{array}$ & $\begin{array}{l}\text { Unnamed hot } \\
\text { spring }\end{array}$ & $\begin{array}{c}\text { Unnamed hot } \\
\text { spring drainage } \\
\text { channel }\end{array}$ & $\begin{array}{c}\text { Unnamed hot } \\
\text { spring drainage } \\
\text { channel }\end{array}$ & $\begin{array}{c}\text { Unnamed hot } \\
\text { spring drainage } \\
\text { channel }\end{array}$ \\
\hline Sample ID & 06WA114D & 06WA115 & 06WA116 & 06WA116B & 06WA116C & 06WA116D \\
\hline Collection Date / Time & $5 / 14 / 0613: 00$ & $5 / 14 / 0615: 45$ & $5 / 14 / 0617: 20$ & $5 / 14 / 0618: 20$ & $5 / 14 / 0618: 20$ & $5 / 14 / 0618: 20$ \\
\hline Temperature, ${ }^{\circ} \mathrm{C}$ & 54.6 & 54.3 & 93.4 & 75.1 & 68.2 & 63.2 \\
\hline pH (field / laboratory) & $9.32 / 9.83$ & $8.70 / 8.91$ & $7.69 / 8.48$ & $7.73 / 8.39$ & $8.07 / 8.46$ & $8.17 / 8.68$ \\
\hline $\begin{array}{l}\text { Specific conductance } \\
\text { (field / laboratory), } \mu \mathrm{S} / \mathrm{cm}\end{array}$ & 1763 / 1800 & 1197 / 1198 & $1320 / 1426$ & $1400 / 1427$ & 1420 / 1454 & 1444 / 1468 \\
\hline Eh, V & -- & 0.275 & 0.083 & -- & --- & --- \\
\hline Density, $\mathrm{g} / \mathrm{mL}$ at $20^{\circ} \mathrm{C}$ & 0.9992 & 0.9990 & 0.9990 & 0.9991 & 0.9990 & 0.9992 \\
\hline Dissolved oxygen (DO), mg/L & --- & 2.5 & --- & --- & --- & --- \\
\hline \multicolumn{7}{|l|}{ Constituent, $\mathrm{mg} / \mathrm{L}^{1}$} \\
\hline Calcium (Ca) & 0.332 & 0.806 & 0.615 & 0.692 & 0.688 & 0.706 \\
\hline Magnesium (Mg) & $<0.001$ & 0.048 & 0.044 & 0.058 & 0.058 & 0.059 \\
\hline Sodium (Na) & 370 & 236 & 289 & 301 & 304 & 315 \\
\hline Potassium (K) & 11.7 & 9.70 & 8.47 & 8.55 & 8.55 & 8.89 \\
\hline Strontium (Sr) & 0.002 & 0.002 & 0.003 & 0.004 & 0.004 & 0.004 \\
\hline Barium (Ba) & $<0.0008$ & $<0.0008$ & $<0.0008$ & $<0.0008$ & $<0.0008$ & $<0.0008$ \\
\hline Lithium (Li) & 4.24 & 2.43 & 2.95 & 3.04 & 3.09 & 3.15 \\
\hline Sulfate $\left(\mathrm{SO}_{4}\right)$ & 23.0 & 16.4 & 12.8 & 12.9 & 14.2 & 12.8 \\
\hline Thiosulfate $\left(\mathrm{S}_{2} \mathrm{O}_{3}\right)$ & $<0.1$ & $<0.1$ & 0.9 & 0.7 & $<0.1$ & $<0.1$ \\
\hline Polythionate $\left(\mathrm{S}_{\mathrm{n}} \mathrm{O}_{6}\right), \mathrm{mM}$ & $<0.001$ & $<0.001$ & $<0.001$ & $<0.001$ & $<0.001$ & $<0.001$ \\
\hline Hydrogen sulfide $\left(\mathrm{H}_{2} \mathrm{~S}\right)$ & --- & $<0.002$ & 0.007 & --- & --- & --- \\
\hline Alkalinity $\left(\mathrm{HCO}_{3}\right)$ & 378 & 252 & 355 & 360 & 365 & 366 \\
\hline Acidity (free / total), mM & --- & --- & --- & --- & --- & --- \\
\hline Fluoride (F) & 30.0 & 21.5 & 21.0 & 22.1 & 22.3 & 22.2 \\
\hline Chloride (Cl) & 295 & 188 & 210 & 208 & 217 & 214 \\
\hline Bromide $(\mathrm{Br})$ & 1.10 & 0.76 & 0.76 & 0.82 & 0.78 & 0.75 \\
\hline Nitrate $\left(\mathrm{NO}_{3}\right)$ & 1.60 & 0.84 & 0.11 & 0.09 & $<0.05$ & $<0.05$ \\
\hline Ammonium $\left(\mathrm{NH}_{4}\right)$ & --- & 0.173 & 0.261 & --- & --- & --- \\
\hline Silica $\left(\mathrm{SiO}_{2}\right)$ & 270 & 187 & 230 & 193 & 195 & 200 \\
\hline Boron (B) & 3.12 & 2.02 & 2.20 & 2.32 & 2.31 & 2.39 \\
\hline Aluminum (Al) & 0.23 & 0.096 & 0.25 & 0.31 & 0.26 & 0.28 \\
\hline Iron total $(\mathrm{Fe}(\mathrm{T}))$ & $<0.002$ & 0.017 & 0.006 & 0.006 & 0.006 & 0.007 \\
\hline Ferrous iron (Fe(II)) & $<0.002$ & $<0.002$ & $<0.002$ & $<0.002$ & $<0.002$ & $<0.002$ \\
\hline Manganese (Mn) & $<0.001$ & $<0.001$ & 0.003 & 0.004 & 0.004 & 0.003 \\
\hline Copper (Cu) & $<0.0005$ & $<0.0005$ & 0.0006 & $<0.0005$ & $<0.0005$ & $<0.0005$ \\
\hline Zinc (Zn) & $<0.004$ & $<0.004$ & $<0.004$ & $<0.004$ & $<0.004$ & $<0.004$ \\
\hline Cadmium (Cd) & $<0.0001$ & $<0.0001$ & $<0.0001$ & $<0.0001$ & $<0.0001$ & $<0.0001$ \\
\hline Chromium (Cr) & $<0.0005$ & $<0.0005$ & $<0.0005$ & $<0.0005$ & $<0.0005$ & $<0.0005$ \\
\hline Cobalt (Co) & $<0.0007$ & $<0.0007$ & $<0.0007$ & $<0.0007$ & $<0.0007$ & $<0.0007$ \\
\hline Mercury total $(\mathrm{Hg}), \mathrm{ng} / \mathrm{L}$ & 25.0 & 30.0 & 174 & 109 & 93.0 & 76.0 \\
\hline Methylmercury $\left(\mathrm{CH}_{3} \mathrm{Hg}\right), \mathrm{ng} / \mathrm{L}$ & -- & --- & -- & -- & --- & -- \\
\hline Nickel (Ni) & $<0.0005$ & $<0.0005$ & $<0.0005$ & $<0.0005$ & $<0.0005$ & $<0.0005$ \\
\hline Lead $(\mathrm{Pb})$ & $<0.0008$ & $<0.0008$ & $<0.0008$ & $<0.0008$ & $<0.0008$ & $<0.0008$ \\
\hline Beryllium (Be) & $<0.001$ & $<0.001$ & 0.001 & 0.001 & 0.001 & 0.001 \\
\hline Vanadium (V) & $<0.005$ & $<0.005$ & $<0.005$ & $<0.005$ & $<0.005$ & $<0.005$ \\
\hline Molybdenum (Mo) & 0.009 & 0.020 & 0.010 & 0.013 & 0.023 & 0.010 \\
\hline Antimony (Sb) & 0.000 & 0.068 & 0.071 & 0.000 & 0.000 & 0.000 \\
\hline Selenium (Se) & $<0.001$ & $<0.001$ & $<0.001$ & $<0.001$ & $<0.001$ & $<0.001$ \\
\hline Arsenic (As) & 1.81 & 1.43 & 1.38 & 1.47 & 1.57 & 1.60 \\
\hline Arsenite (As(III)) & $0.027^{2}$ & $0.018^{2}$ & 0.111 & 0.061 & 0.036 & 0.025 \\
\hline Dissolved organic carbon (DOC) & --- & 2.1 & 3.0 & --- & --- & --- \\
\hline$\delta \mathrm{D}$, per mil & -138.90 & -142.30 & -142.87 & -141.28 & -140.22 & -140.75 \\
\hline$\delta^{18} 0$, per mil & -15.01 & -16.77 & -16.83 & -16.52 & -16.39 & -16.22 \\
\hline sum cations, meq/L & 16.7 & 10.8 & 13.2 & 13.7 & 13.8 & 14.3 \\
\hline sum anions, meq/L & 16.3 & 10.9 & 13.1 & 13.1 & 13.5 & 13.4 \\
\hline Charge imbalance, percent & 2.4 & -0.5 & 0.5 & 4.3 & 2.5 & 6.7 \\
\hline
\end{tabular}


Table 12. Results of water analyses for the Mud Volcano area, 2006-2008.

[ $\delta \mathrm{D}$ and $\delta 18 \mathrm{O}$, isotopic composition of hydrogen and oxygen relative to VSMOW; m, meters; meq/L, milliequivalents per liter; $\mathrm{mg} / \mathrm{L}$, milligrams per liter; $\mathrm{mM}$, millimolar; $\mu \mathrm{S} / \mathrm{cm}$, microsiemens per centimeter; $\mathrm{ng} / \mathrm{L}$ nanograms per liter; V, volts; ---, not measured; ${ }^{\circ} \mathrm{C}$, degrees Celsius; <, less than]

\begin{tabular}{|c|c|c|c|c|c|}
\hline Location & Sulphur Caldron & Sulphur Caldron & Sulphur Caldron & Turbulent Pool & Turbulent Pool \\
\hline Sample ID & 06WA164 & 07WA110 & 08WA114 & 06WA165 & 07WA109 \\
\hline Collection Date / Time & $9 / 17 / 0614: 43$ & 9/11/07 11:45 & $9 / 19 / 0810: 00$ & $9 / 17 / 0616: 30$ & 9/11/07 11:00 \\
\hline Temperature, ${ }^{\circ} \mathrm{C}$ & 73.5 & 73.9 & 73.2 & 58.5 & 59.3 \\
\hline pH (field / laboratory) & $2.02 / 1.84$ & $1.71 / 1.82$ & $1.60 / 1.58$ & $1.89 / 1.84$ & $1.99 / 2.00$ \\
\hline $\begin{array}{l}\text { Specific conductance } \\
\text { (field / laboratory), } \mu \mathrm{S} / \mathrm{cm}\end{array}$ & $3950 / 7880$ & $6480 / 12,410$ & $6640 / 12,090$ & $4360 / 7070$ & $3660 / 5560$ \\
\hline Eh, V & 0.128 & 0.088 & --- & 0.071 & 0.017 \\
\hline Density, $\mathrm{g} / \mathrm{mL}$ at $20^{\circ} \mathrm{C}$ & 1.0011 & 1.0014 & 1.0009 & 1.0000 & 0.9996 \\
\hline Dissolved oxygen (DO), mg/L & --- & --- & --- & --- & --- \\
\hline \multicolumn{6}{|l|}{ Constituent, $\mathrm{mg} / \mathrm{L}^{1}$} \\
\hline Calcium (Ca) & 26.5 & 39.3 & 36.8 & 11.6 & 13.1 \\
\hline Magnesium (Mg) & 8.94 & 10.5 & 9.12 & 3.77 & 5.94 \\
\hline Sodium $(\mathrm{Na})$ & 21.0 & 34.6 & 29.4 & 15.5 & 18.8 \\
\hline Potassium (K) & 19.0 & 26.0 & 29.2 & 13.9 & 11.6 \\
\hline Strontium (Sr) & 0.113 & 0.174 & 0.182 & 0.114 & 0.139 \\
\hline Barium (Ba) & 0.045 & 0.061 & 0.080 & 0.052 & 0.055 \\
\hline Lithium (Li) & 0.024 & 0.025 & 0.030 & 0.007 & 0.012 \\
\hline Sulfate $\left(\mathrm{SO}_{4}\right)$ & 2580 & 3620 & 3490 & 1490 & 1370 \\
\hline Thiosulfate $\left(\mathrm{S}_{2} \mathrm{O}_{3}\right)$ & $<0.1$ & $<0.1$ & --- & $<0.1$ & $<0.1$ \\
\hline Polythionate $\left(\mathrm{S}_{\mathrm{n}} \mathrm{O}_{6}\right), \mathrm{mM}$ & $<0.001$ & $<0.001$ & $<0.001$ & $<0.001$ & $<0.001$ \\
\hline Hydrogen sulfide $\left(\mathrm{H}_{2} \mathrm{~S}\right)$ & 0.016 & 0.019 & --- & --- & 0.04 \\
\hline Alkalinity $\left(\mathrm{HCO}_{3}\right)$ & --- & --- & --- & --- & --- \\
\hline Acidity (free / total), mM & $43.5 / 46.0$ & $44.5 / 60.0$ & $35.3 / 45.6$ & $29.1 / 30.0$ & $17.8 / 23.3$ \\
\hline Fluoride (F) & 0.310 & 0.590 & 0.488 & 0.660 & 0.530 \\
\hline Chloride (Cl) & 4.12 & 7.52 & 2.86 & 4.80 & 7.01 \\
\hline Bromide (Br) & $<0.03$ & $<0.03$ & 0.03 & $<0.03$ & $<0.03$ \\
\hline Nitrate $\left(\mathrm{NO}_{3}\right)$ & $<0.05$ & $<0.05$ & 0.26 & $<0.05$ & $<0.05$ \\
\hline Ammonium $\left(\mathrm{NH}_{4}\right)$ & 59.0 & 62.7 & 86.6 & 15.2 & 14.2 \\
\hline Silica $\left(\mathrm{SiO}_{2}\right)$ & 412 & 332 & 291 & 243 & 249 \\
\hline Boron (B) & 3.97 & 2.61 & 3.28 & 1.05 & 0.850 \\
\hline Aluminum (Al) & 203 & 131 & 81.6 & 37.8 & 41.7 \\
\hline Iron total $(\mathrm{Fe}(\mathrm{T}))$ & 22.8 & 31.8 & 20.4 & 6.74 & 20.0 \\
\hline Ferrous iron (Fe(II)) & 22.6 & 31.4 & 19.5 & 6.71 & 20.0 \\
\hline Manganese (Mn) & 0.411 & 0.579 & 0.604 & 0.304 & 0.376 \\
\hline Copper $(\mathrm{Cu})$ & 0.0013 & 0.0018 & 0.0005 & 0.0079 & $<0.0005$ \\
\hline Zinc (Zn) & 0.205 & 0.303 & 0.174 & 0.069 & 0.097 \\
\hline Cadmium (Cd) & 0.0005 & 0.0006 & 0.0003 & 0.0001 & 0.0002 \\
\hline Chromium (Cr) & 0.089 & 0.079 & 0.045 & 0.014 & 0.028 \\
\hline Cobalt (Co) & 0.0040 & 0.0085 & 0.0046 & 0.0020 & 0.0040 \\
\hline Mercury total (Hg), ng/L & 127 & 210 & 65.0 & 80.0 & 39.0 \\
\hline Methylmercury $\left(\mathrm{CH}_{3} \mathrm{Hg}\right), \mathrm{ng} / \mathrm{L}$ & 0.78 & 10.3 & 9.30 & 5.04 & 5.82 \\
\hline Nickel (Ni) & 0.043 & 0.050 & 0.028 & 0.0094 & 0.016 \\
\hline Lead $(\mathrm{Pb})$ & 0.0030 & 0.018 & 0.025 & 0.0058 & 0.0086 \\
\hline Beryllium (Be) & 0.004 & 0.007 & 0.006 & 0.002 & 0.002 \\
\hline Vanadium (V) & 0.044 & 0.045 & 0.039 & 0.011 & 0.029 \\
\hline Molybdenum (Mo) & $<0.007$ & $<0.007$ & $<0.007$ & $<0.007$ & $<0.007$ \\
\hline Antimony (Sb) & $<0.0005$ & 0.006 & $<0.001$ & $<0.0005$ & 0.006 \\
\hline Selenium (Se) & 0.005 & 0.001 & $<0.001$ & 0.001 & $<0.001$ \\
\hline Arsenic (As) & 0.005 & 0.085 & 0.0037 & 0.0013 & 0.035 \\
\hline Arsenite (As(III)) & 0.002 & 0.004 & 0.0027 & $<0.001$ & 0.001 \\
\hline Dissolved organic carbon (DOC) & 7.7 & 5.8 & 5.8 & 4.0 & 6.1 \\
\hline$\delta \mathrm{D}$, per mil & -108.43 & -106.03 & -108.69 & -105.13 & -102.14 \\
\hline$\delta^{18} 0$, per mil & -8.77 & -7.80 & -8.37 & -7.80 & -7.55 \\
\hline sum cations, meq/L & 27.5 & 38.1 & 43.3 & 19.3 & 16.9 \\
\hline sum anions, meq/L & 27.5 & 40.8 & 39.9 & 19.5 & 18.1 \\
\hline Charge imbalance, percent & 0.2 & -7.0 & 8.2 & -0.8 & -7.0 \\
\hline
\end{tabular}


Table 13. Results of water analyses for Washburn Hot Springs, 2006-2008.

[ $\delta \mathrm{D}$ and $\delta 18 \mathrm{O}$, isotopic composition of hydrogen and oxygen relative to VSMOW; m, meters; meq/L, milliequivalents per liter; $\mathrm{mg} / \mathrm{L}$, milligrams per liter; $\mathrm{mM}$, millimolar; $\mu \mathrm{S} / \mathrm{cm}$, microsiemens per centimeter; $\mathrm{ng} / \mathrm{L}$ nanograms per liter; V, volts; ---, not measured; ${ }^{\circ} \mathrm{C}$, degrees Celsius; <, less than]

\begin{tabular}{|c|c|c|c|c|}
\hline Location & Inkpot \# 1 & Inkpot \#3 & $\begin{array}{c}\text { Unnamed, } 160 \text { feet } \\
\text { elevation above inkpots }\end{array}$ & Upper large hot pool \\
\hline Sample ID & 06WA166 & 06WA167 & 06WA169 & 06WA168 \\
\hline Collection Date / Time & 9/18/06 11:45 & $9 / 18 / 0613: 25$ & $9 / 18 / 0616: 32$ & 9/18/06 14:45 \\
\hline Temperature, ${ }^{\circ} \mathrm{C}$ & 80.2 & 70.9 & 88.2 & 74.5 \\
\hline pH (field / laboratory) & $6.53 / 7.69$ & $3.25 / 2.88$ & $7.33 / 8.06$ & $6.64 / 7.47$ \\
\hline \multicolumn{5}{|l|}{ Specific conductance } \\
\hline (field / laboratory), $\mu \mathrm{S} / \mathrm{cm}$ & $3140 / 3470$ & $5750 / 6730$ & $1330 / 1540$ & $3450 / 3710$ \\
\hline Eh, V & -0.164 & -0.037 & -0.078 & -0.146 \\
\hline Density, $\mathrm{g} / \mathrm{mL}$ at $20^{\circ} \mathrm{C}$ & 0.9997 & 1.0010 & 0.9988 & 0.9998 \\
\hline Dissolved oxygen (DO), mg/L & --- & --- & --- & --- \\
\hline \multicolumn{5}{|l|}{ Constituent, mg/L ${ }^{1}$} \\
\hline Calcium (Ca) & 24.0 & 53.4 & 2.76 & 28.7 \\
\hline Magnesium (Mg) & 11.0 & 26.9 & 1.80 & 13.7 \\
\hline Sodium $(\mathrm{Na})$ & 34.2 & 37.8 & 3.45 & 29.6 \\
\hline Potassium (K) & 13.5 & 14.4 & 3.09 & 12.4 \\
\hline Strontium (Sr) & 0.146 & 0.225 & 0.029 & 0.167 \\
\hline Barium (Ba) & 0.042 & 0.024 & 0.049 & 0.041 \\
\hline Lithium (Li) & 0.011 & 0.045 & $<0.001$ & 0.005 \\
\hline Sulfate $\left(\mathrm{SO}_{4}\right)$ & 1360 & 2710 & 473 & 1330 \\
\hline Thiosulfate $\left(\mathrm{S}_{2} \mathrm{O}_{3}\right)$ & 2.1 & $<0.1$ & 7.4 & 0.5 \\
\hline Polythionate $\left(\mathrm{S}_{\mathrm{n}} \mathrm{O}_{6}\right), \mathrm{mM}$ & 0.002 & $<0.001$ & $<0.001$ & $<0.001$ \\
\hline Hydrogen sulfide $\left(\mathrm{H}_{2} \mathrm{~S}\right)$ & 0.11 & 0.40 & 0.003 & 0.006 \\
\hline Alkalinity $\left(\mathrm{HCO}_{3}\right)$ & 137 & --- & 82.2 & 203 \\
\hline Acidity (free / total), mM & --- & $2.71 / 4.19$ & --- & --- \\
\hline Fluoride (F) & 0.650 & 0.790 & 0.05 & 0.400 \\
\hline Chloride (Cl) & 1.61 & 1.49 & 0.35 & 1.67 \\
\hline Bromide (Br) & $<0.03$ & $<0.03$ & $<0.03$ & $<0.03$ \\
\hline Nitrate $\left(\mathrm{NO}_{3}\right)$ & 0.09 & 0.06 & $<0.05$ & $<0.05$ \\
\hline Ammonium $\left(\mathrm{NH}_{4}\right)$ & 439 & 837 & 178 & 477 \\
\hline Silica $\left(\mathrm{SiO}_{2}\right)$ & 124 & 268 & 84.3 & 130 \\
\hline Boron (B) & 8.67 & 11.7 & 2.95 & 6.40 \\
\hline Aluminum (Al) & 0.053 & 11.3 & 0.26 & 0.064 \\
\hline Iron total $(\mathrm{Fe}(\mathrm{T}))$ & 0.010 & 5.93 & 0.010 & 0.006 \\
\hline Ferrous iron (Fe(II)) & 0.010 & 5.93 & 0.010 & 0.006 \\
\hline Manganese (Mn) & 0.173 & 0.773 & 0.042 & 0.241 \\
\hline Copper (Cu) & $<0.0005$ & $<0.0005$ & 0.0013 & $<0.0005$ \\
\hline Zinc (Zn) & $<0.004$ & 0.083 & $<0.004$ & $<0.004$ \\
\hline Cadmium (Cd) & $<0.0001$ & $<0.0001$ & $<0.0001$ & $<0.0001$ \\
\hline Chromium (Cr) & 0.0015 & 0.0083 & 0.0006 & 0.0020 \\
\hline Cobalt (Co) & $<0.0007$ & $<0.0007$ & $<0.0007$ & $<0.0007$ \\
\hline Mercury total (Hg), ng/L & 447 & 26.0 & 494 & 75.0 \\
\hline Methylmercury $\left(\mathrm{CH}_{3} \mathrm{Hg}\right), \mathrm{ng} / \mathrm{L}$ & 0.09 & 0.04 & 0.09 & 0.04 \\
\hline Nickel (Ni) & $<0.0005$ & $<0.0005$ & $<0.0005$ & $<0.0005$ \\
\hline Lead $(\mathrm{Pb})$ & $<0.0008$ & $<0.0008$ & $<0.0008$ & $<0.0008$ \\
\hline Beryllium (Be) & $<0.001$ & 0.006 & $<0.001$ & $<0.001$ \\
\hline Vanadium $(\mathrm{V})$ & $<0.005$ & 0.026 & 0.009 & $<0.005$ \\
\hline Molybdenum (Mo) & 0.007 & 0.014 & $<0.007$ & 0.008 \\
\hline Antimony (Sb) & $<0.0005$ & $<0.0005$ & $<0.0005$ & $<0.0005$ \\
\hline Selenium (Se) & 0.001 & $<0.001$ & $<0.001$ & 0.001 \\
\hline Arsenic (As) & 0.0017 & $<0.0005$ & 0.004 & 0.0007 \\
\hline Arsenite (As(III)) & $<0.0005$ & $<0.0005$ & 0.001 & $<0.0005$ \\
\hline Dissolved organic carbon (DOC) & --- & 5.4 & 2.3 & 7.0 \\
\hline$\delta \mathrm{D}$, per mil & -110.40 & -110.61 & -119.65 & -110.62 \\
\hline$\delta^{18} 0$, per mil & -3.07 & -3.76 & -7.42 & -2.39 \\
\hline sum cations, meq/L & 24.7 & 47.5 & 7.2 & 26.7 \\
\hline sum anions, meq/L & 27.9 & 46.9 & 10.8 & 28.1 \\
\hline Charge imbalance, percent & -12.1 & 1.4 & -40.1 & -4.9 \\
\hline
\end{tabular}




\section{Quality Assurance and Quality Control}

Several techniques were used to assure the quality of the analytical data. These techniques included calculation of charge imbalance (C.I.), analysis of USGS standard reference water samples (SRWS), replicate determinations in the laboratory, spike recovery determinations, and determination by alternative analytical methods.

The charge-balance calculation is one of the first and simplest of quality-assurance/qualitycontrol procedures to check the accuracy of a water analysis. It is based on the principle that an aqueous solution containing electrolytes, or charged ions, must be electrically neutral overall. The equivalent concentration of cations must equal that of anions:

$$
\sum_{i} i_{i} \quad 0 \quad \sum_{i}^{+}-\sum_{i} c_{i}^{-}
$$

where $c_{i}=$ the equivalent concentration (usually in milliequivalents per liter) for the $i$ th ion, $c_{i}^{+}=$ the equivalent concentration for the $i^{\text {th }}$ cation, and $c_{i}^{-}=$the equivalent concentration for the $i^{\text {th }}$ anion. Before an accurate charge balance can be calculated for acid waters, equivalent hydrogenion concentrations must be calculated based on the $\mathrm{pH}$ measurement and thermodynamic speciation calculations (see the Revised $\mathrm{pH}$ Measurements section). The charge balance calculation is discussed in more detail by Ball and others (2006).

Major cations and anions were analyzed for 104 samples and the data were checked for C.I. using the geochemical code WATEQ4F (Ball and Nordstrom, 1991). WATEQ4F uses equation 10 to calculate C.I.:

$$
\text { C.I. }(\text { percent })=\frac{100 \times(\text { sum cations }- \text { sum anions })}{(\text { sum cations }+ \text { sum anions })}
$$

where sum cations and sum anions are in meq/L.

The C.I., sum cations (meq/L), and sum anions (meq/L) are reported in tables 5-13 for samples having major cation and anion determinations. A frequency plot of C.I. for all samples with complete analyses is shown in figure 30 . The Gaussian fit C.I. mean is -0.9 percent with a standard deviation of 5.0 percent. Analyses having a C.I. less than \pm 10 percent are considered reliable for speciation calculations (Nordstrom and Munoz, 1994). Two sample analyses out of 104 had a C.I. (-12.1 and -40.1 percent) greater than \pm 10 percent. 


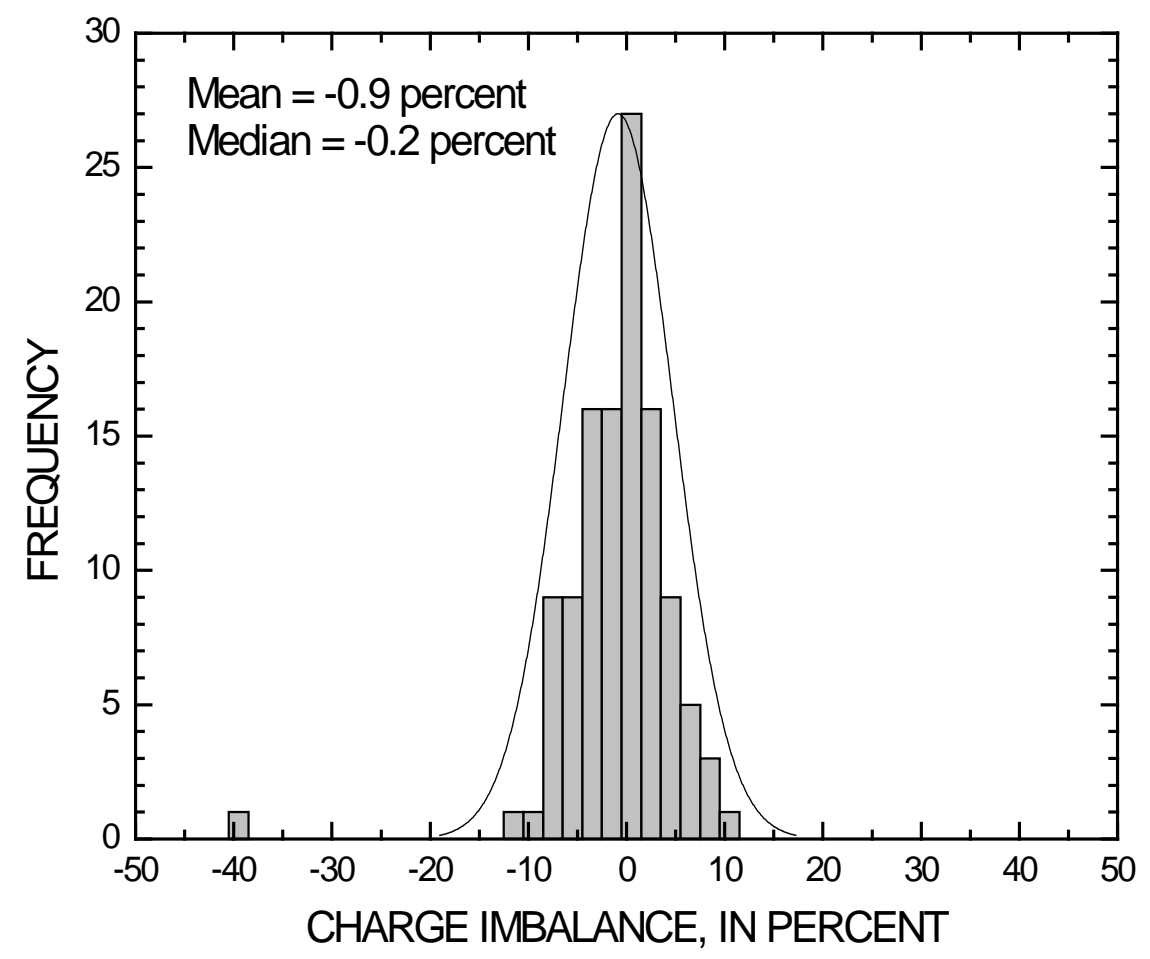

Figure 30. Frequency distribution of charge imbalance for samples having major cation and anion determinations.

U.S. Geological Survey SRWSs were analyzed as unknowns along with the samples to check for accuracy. Standard reference water samples AMW4, M150, M156, M158, M172, M182, T163, T179, T181, T183, T189, Hg7, and Hg22 were used to check the analytical methods for major and trace metals, anions, and $\mathrm{Hg}$ (Farrar, 2000; Connor and others, 2001). The SRWSs were analyzed several times during each analytical run. For each SRWS constituent, the analytical result, the most probable value (MPV), and the percent difference are presented in table 14 . Additional information about the USGS SRWS program can be obtained at URL http://bqs.usgs.gov/srs.

Replicate determinations were performed for all methods and all constituents. Samples were diluted as necessary to bring the analyte concentration within the optimal range of the method and for each dilution at least two separate aliquots of each sample were analyzed. Replicate determinations minimize errors owing to dilution, contamination, and analytical imprecision. Reported concentrations are the means, excluding outliers, of the replicate determinations.

Concentrations of F, Al, $\mathrm{Fe}(\mathrm{T}), \mathrm{Mn}, \mathrm{Cu}, \mathrm{Cd}, \mathrm{Cr}, \mathrm{Co}, \mathrm{Ni}, \mathrm{Pb}, \mathrm{Sb}(\mathrm{T})$, Se, and $\mathrm{As}(\mathrm{T})$ were determined by more than one method. Comparing analytical results from alternative methods can serve as an accuracy check, although in all cases one method was preferred over the other depending on sample matrix and proximity to the method detection limit. Concentrations of $\mathrm{Al}$, $\mathrm{As}(\mathrm{T}), \mathrm{Fe}(\mathrm{T}), \mathrm{Cd}, \mathrm{Co}, \mathrm{Cr}, \mathrm{Cu}, \mathrm{Ni}, \mathrm{Pb}, \mathrm{Sb}(\mathrm{T})$, and $\mathrm{Se}$ were determined by ICP-OES as well as by the preferred HGAAS method for $\mathrm{As}(\mathrm{T})$ and $\mathrm{Sb}(\mathrm{T})$, FerroZine method for $\mathrm{Fe}(\mathrm{T})$, and GFAAS for 
$\mathrm{Al}, \mathrm{Cd}, \mathrm{Co}, \mathrm{Cr}, \mathrm{Cu}, \mathrm{Pb}$, and $\mathrm{Se}$. Concentrations of $\mathrm{Al}$ were measured by GFAAS only for samples that were below the ICP-OES detection limit and $\mathrm{Cd}, \mathrm{Co}, \mathrm{Cr}, \mathrm{Cu}, \mathrm{Pb}$, and Se concentrations were near or below the ICP-OES detection limit for nearly all samples. Therefore, for these constituents comparison with the preferred method was of little value. The data reported in tables 5-13 were obtained using the preferred method unless there was insufficient sample volume to perform the determinations.

Comparison of analytical results from alternative methods for $\mathrm{F}$, total dissolved As, and total dissolved Fe are shown in figures $31 \mathrm{~A}$ to $\mathrm{C}$. The uncertainty line was calculated based on the method with the higher detection limit. At the method detection limit the uncertainty is \pm 100 percent and decreases to \pm 5 percent at 20 times the detection limit (quantitation limit).

Fluoride concentrations were determined by both IC and ISE (fig. 31A). Samples for F determination by ISE were mixed 1:1 with a total ionic strength adjustment buffer (TISAB III; Barnard and Nordstrom, 1980), which minimizes the effect of Al-F complexing on the analytical result. However, several of the samples that contained Al at concentrations greater than $5 \mathrm{mg} / \mathrm{L}$ yielded F concentrations by ISE that were substantially lower than those obtained by IC, suggesting that IC determinations for those samples were more accurate. For samples containing greater than $5 \mathrm{mg} / \mathrm{L} \mathrm{Al}$, the $\mathrm{F}$ concentrations determined by IC are reported.

Concentrations of As by HGAAS and Fe by FerroZine were determined on a subsample preserved with $\mathrm{HCl}$, whereas As and $\mathrm{Fe}$ by ICP-OES were determined on a subsample preserved with $\mathrm{HNO}_{3}$. Arsenic concentrations determined by ICP-OES were frequently higher than HGAAS. The difference in the As measurements most likely results from the difference in sample preservation. Samples containing high $\mathrm{S}^{2-}$ concentrations may form As-S precipitates when preserved with $\mathrm{HCl}$ (Planer-Friedrich and others, 2007). With only a few exceptions, $\mathrm{Fe}$ concentrations determined by FerroZine and by ICP-OES are in good agreement. The largest difference in Fe concentration was for a sample from Washburn Hot Springs (06WA135). Results for $\mathrm{As}$ and $\mathrm{Fe}$ are reported for the sample preserved with $\mathrm{HCl}$ because the redox species concentrations were obtained from this split. Considering the uncertainty of the measurements and that the analyses were performed on different splits, analytical results for total dissolved As and total dissolved $\mathrm{Fe}$ obtained by the alternative methods are in good agreement. 
Table 14. Measurement of concentrations of dissolved constituents in standard reference water samples.

[CVAFS, cold vapor atomic fluorescence spectrometry; GFAAS, graphite furnace atomic absorption spectrometry; HGAAS, hydride generation atomic absorption spectrometry; IC, ion chromatography; ICP-OES, inductively coupled plasma-optical emission spectrometry; ISE, ion selective electrode; $\mathrm{mg} / \mathrm{L}$, milligrams per liter; $\mathrm{n}$, number of analyses; ng/L, nanograms per liter; s, standard deviation; SRWS, standard reference water sample; USGS, U.S. Geological Survey; \%RSD, percent relative standard deviation; <, less than; ---, no data]

\begin{tabular}{|c|c|c|c|c|c|c|c|c|}
\hline Analyte & $\begin{array}{c}\text { Analytical } \\
\text { Method }\end{array}$ & $\begin{array}{r}\text { USGS } \\
\text { SRWS } \\
\end{array}$ & $\mathbf{n}$ & $\mathrm{mg} / \mathrm{L}^{\mathrm{a}}$ & $\mathbf{s}$ & $\begin{array}{c}\text { Percent } \\
\text { RSD } \\
\end{array}$ & $\begin{array}{c}\text { Most Probable } \\
\text { Value, mg/L }\end{array}$ & $\begin{array}{c}\text { Percent } \\
\text { Difference }\end{array}$ \\
\hline Calcium (Ca) & ICP-OES & T179 & 10 & 18.3 & 0.4 & 2 & $18.9 \pm 0.615$ & -3 \\
\hline Calcium (Ca) & ICP-OES & $\mathrm{T} 181$ & 14 & 13.2 & 0.8 & 6 & $13.4 \pm 0.556$ & -1 \\
\hline Calcium (Ca) & ICP-OES & $\mathrm{T} 183$ & 12 & 11.3 & 0.4 & 3 & $11.6 \pm 0.423$ & -2 \\
\hline Calcium (Ca) & ICP-OES & T189 & 16 & 29.7 & 1.2 & 4 & $30.6 \pm 1.11$ & -3 \\
\hline Magnesium (Mg) & ICP-OES & $\mathrm{T} 179$ & 7 & 4.55 & 0.12 & 3 & $4.59 \pm 0.185$ & -1 \\
\hline Magnesium (Mg) & ICP-OES & T181 & 9 & 3.07 & 0.27 & 9 & $3.05 \pm 0.115$ & 1 \\
\hline Magnesium (Mg) & ICP-OES & T183 & 13 & 7.73 & 0.25 & 3 & $7.89 \pm 0.341$ & -2 \\
\hline Magnesium (Mg) & ICP-OES & T189 & 13 & 8.06 & 0.19 & 2 & $8.13 \pm 0.256$ & -1 \\
\hline Sodium (Na) & ICP-OES & $\mathrm{T} 179$ & 14 & 26.0 & 1.3 & 5 & $26.2 \pm 1.00$ & -1 \\
\hline Sodium (Na) & ICP-OES & $\mathrm{T} 181$ & 12 & 15.0 & 0.4 & 2 & $15.0 \pm 0.519$ & 0 \\
\hline Sodium (Na) & ICP-OES & $\mathrm{T} 183$ & 16 & 12.8 & 0.6 & 5 & $12.7 \pm 0.530$ & 1 \\
\hline Sodium (Na) & ICP-OES & T189 & 15 & 48.5 & 2.2 & 5 & $50.0 \pm 1.89$ & -3 \\
\hline Potassium (K) & ICP-OES & $\mathrm{T} 179$ & 15 & 1.43 & 0.12 & 8 & $1.40 \pm 0.148$ & 2 \\
\hline Potassium (K) & ICP-OES & $\mathrm{T} 181$ & 13 & 1.40 & 0.05 & 4 & $1.52 \pm 0.090$ & -8 \\
\hline Potassium (K) & ICP-OES & T183 & 18 & 2.14 & 0.16 & 7 & $2.57 \pm 0.096$ & -17 \\
\hline Potassium (K) & ICP-OES & T189 & 20 & 3.63 & 0.21 & 6 & $3.84 \pm 0.193$ & -5 \\
\hline Strontium (Sr) & ICP-OES & $\mathrm{T} 179$ & 9 & 0.115 & 0.002 & 2 & $0.113 \pm 0.00404$ & 2 \\
\hline Strontium (Sr) & ICP-OES & $\mathrm{T} 181$ & 9 & 0.083 & 0.003 & 4 & $0.0816 \pm 0.00319$ & 2 \\
\hline Strontium (Sr) & ICP-OES & T183 & 13 & 0.084 & 0.002 & 3 & $0.0824 \pm 0.00248$ & 2 \\
\hline Strontium (Sr) & ICP-OES & T189 & 15 & 0.188 & 0.004 & 2 & $0.187 \pm 0.00519$ & 1 \\
\hline Barium (Ba) & ICP-OES & $\mathrm{T} 179$ & 6 & 0.036 & 0.001 & 3 & $0.036 \pm 0.0017$ & 0 \\
\hline Barium (Ba) & ICP-OES & $\mathrm{T} 181$ & 6 & 0.025 & 0.001 & 4 & $0.0255 \pm 0.00126$ & 0 \\
\hline Barium (Ba) & ICP-OES & T183 & 13 & 0.030 & 0.001 & 3 & $0.0299 \pm 0.0013$ & -1 \\
\hline Barium (Ba) & ICP-OES & T189 & 15 & 0.070 & 0.001 & 2 & $0.0715 \pm 0.00208$ & -2 \\
\hline Lithium (Li) & ICP-OES & $\mathrm{T} 179$ & 14 & 0.006 & 0.001 & 9 & $0.0065 \pm 0.000434$ & -1 \\
\hline Lithium (Li) & ICP-OES & T181 & 15 & 0.008 & 0.001 & 8 & $0.0084 \pm 0.00056$ & -6 \\
\hline Lithium (Li) & ICP-OES & T183 & 18 & 0.003 & 0.001 & 43 & $0.00333 \pm 0.000222$ & 4 \\
\hline Lithium (Li) & ICP-OES & T189 & 19 & 0.005 & 0.000 & 7 & $0.00395 \pm 0.000289$ & 17 \\
\hline Sulfate $\left(\mathrm{SO}_{4}\right)$ & IC & M150 & 2 & 5.57 & 0.16 & 2.8 & $5.50 \pm 0.54$ & 1 \\
\hline Sulfate $\left(\mathrm{SO}_{4}\right)$ & IC & M156 & 8 & 42.2 & 0.4 & 0.9 & $43.0 \pm 1.78$ & -2 \\
\hline Sulfate $\left(\mathrm{SO}_{4}\right)$ & IC & M172 & 6 & 12.2 & 0.4 & 3.0 & $12.2 \pm 0.604$ & 0 \\
\hline Sulfate $\left(\mathrm{SO}_{4}\right)$ & IC & M182 & 24 & 10.2 & 0.2 & 2.0 & $10.3 \pm 0.382$ & -1 \\
\hline
\end{tabular}


Table 14. Measurement of concentrations of dissolved constituents in standard reference water samplesContinued.

\begin{tabular}{|c|c|c|c|c|c|c|c|c|}
\hline Analyte & $\begin{array}{l}\text { Analytical } \\
\text { Method }\end{array}$ & \begin{tabular}{|l|} 
USGS \\
SRWS
\end{tabular} & n & $\mathrm{mg} / \mathbf{L}^{\mathrm{a}}$ & $\mathbf{s}$ & $\begin{array}{c}\text { Percent } \\
\text { RSD }\end{array}$ & $\begin{array}{l}\text { Most Probable } \\
\text { Value, mg/L }\end{array}$ & $\begin{array}{c}\text { Percent } \\
\text { Difference }\end{array}$ \\
\hline Alkalinity $\left(\mathrm{HCO}_{3}\right)$ & Titration & M156 & 15 & 73.5 & 1.2 & 1.6 & $75.4 \pm 3.1$ & -3 \\
\hline Alkalinity $\left(\mathrm{HCO}_{3}\right)$ & Titration & M158 & 1 & 75.6 & --- & --- & $77.6 \pm 3.1$ & -2 \\
\hline Alkalinity $\left(\mathrm{HCO}_{3}\right)$ & Titration & M182 & 14 & 41.3 & 0.6 & 1.5 & $42.6 \pm 1.9$ & -3 \\
\hline Fluoride $(\mathrm{F})$ & IC & M156 & 6 & 0.57 & 0.06 & 11.4 & $0.523 \pm 0.045$ & 9 \\
\hline Fluoride $(\mathrm{F})$ & IC & M172 & 4 & 0.37 & 0.04 & 11.4 & $0.381 \pm 0.027$ & -2 \\
\hline Fluoride (F) & IC & M182 & 16 & 0.18 & 0.01 & 3.9 & $0.160 \pm 0.022$ & 11 \\
\hline Fluoride (F) & ISE & M172 & 3 & 0.373 & 0.008 & 2.3 & $0.381 \pm 0.027$ & -2 \\
\hline Fluoride (F) & ISE & M182 & 3 & 0.173 & 0.004 & 2.4 & $0.160 \pm 0.022$ & 8 \\
\hline Chloride (Cl) & IC & M150 & 2 & 19.0 & 0.3 & 1.5 & $17.0 \pm 1.5$ & 12 \\
\hline Chloride (Cl) & IC & M156 & 6 & 67.4 & 1.8 & 2.7 & $64.7 \pm 1.9$ & 4 \\
\hline Chloride (Cl) & IC & M172 & 6 & 15.5 & 0.2 & 1.4 & $16.0 \pm 0.37$ & -3 \\
\hline Chloride (Cl) & IC & M182 & 23 & 21.2 & 0.6 & 2.6 & $21.3 \pm 0.482$ & 0 \\
\hline Bromide (Br) & IC & AMW4 & 16 & 0.41 & 0.04 & 9.1 & $0.430 \pm 0.037$ & -6 \\
\hline Silica $\left(\mathrm{SiO}_{2}\right)$ & ICP-OES & T179 & 14 & 14.9 & 0.5 & 3 & $14.9 \pm 0.604$ & 0 \\
\hline Silica $\left(\mathrm{SiO}_{2}\right)$ & ICP-OES & $\mathrm{T} 181$ & 14 & 13.0 & 0.4 & 3 & $13.1 \pm 0.519$ & -1 \\
\hline Silica $\left(\mathrm{SiO}_{2}\right)$ & ICP-OES & $\mathrm{T} 183$ & 18 & 8.52 & 0.47 & 6 & $8.27 \pm 0.448$ & 3 \\
\hline Silica $\left(\mathrm{SiO}_{2}\right)$ & ICP-OES & T189 & 21 & 12.6 & 0.6 & 5 & $12.2 \pm 0.667$ & 3 \\
\hline Boron (B) & ICP-OES & $\mathrm{T} 179$ & 11 & 0.026 & 0.003 & 12 & $0.028 \pm 0.00182$ & -8 \\
\hline Boron (B) & ICP-OES & $\mathrm{T} 181$ & 9 & 0.019 & 0.002 & 9 & $0.021 \pm 0.00156$ & -11 \\
\hline Boron (B) & ICP-OES & T183 & 11 & 0.057 & 0.004 & 7 & $0.0606 \pm 0.00311$ & -6 \\
\hline Boron (B) & ICP-OES & T189 & 11 & 0.019 & 0.003 & 17 & $0.021 \pm 0.00289$ & -10 \\
\hline Aluminum (Al) & ICP-OES & T179 & 10 & 0.054 & 0.016 & 29 & $0.0517 \pm 0.00319$ & 4 \\
\hline Aluminum (Al) & ICP-OES & $\mathrm{T} 181$ & 9 & $<0.08$ & --- & --- & $0.0162 \pm 0.00119$ & --- \\
\hline Aluminum (Al) & ICP-OES & $\mathrm{T} 183$ & 14 & 0.122 & 0.013 & 10 & $0.127 \pm 0.0089$ & -4 \\
\hline Aluminum (Al) & ICP-OES & T189 & 21 & $<0.08$ & --- & --- & $0.0167 \pm 0.00114$ & --- \\
\hline Aluminum (Al) & GFAAS & T179 & 4 & 0.052 & 0.002 & 3.1 & $0.517 \pm 0.00319$ & 0.0 \\
\hline Aluminum (Al) & GFAAS & $\mathrm{T} 181$ & 4 & 0.015 & 0.000 & 3.3 & $0.162 \pm 0.00119$ & -8.7 \\
\hline Aluminum (Al) & GFAAS & $\mathrm{T} 183$ & 4 & 0.127 & 0.013 & 10 & $0.127 \pm 0.0089$ & 0.0 \\
\hline Iron $(\mathrm{Fe})$ & Colorimetric & $\mathrm{T} 179$ & 2 & 0.121 & 0.003 & 2.5 & $0.125 \pm 0.00815$ & -3 \\
\hline Iron $(\mathrm{Fe})$ & Colorimetric & $\mathrm{T} 183$ & 4 & 0.206 & 0.003 & 1.2 & $0.206 \pm 0.0155$ & 0 \\
\hline Iron (Fe) & ICP-OES & $\mathrm{T} 179$ & 9 & 0.122 & 0.005 & 4 & $0.125 \pm 0.00815$ & -2 \\
\hline Iron (Fe) & ICP-OES & $\mathrm{T} 181$ & 9 & 0.121 & 0.009 & 8 & $0.119 \pm 0.0089$ & 2 \\
\hline Iron $(\mathrm{Fe})$ & ICP-OES & T183 & 13 & 0.198 & 0.009 & 4 & $0.206 \pm 0.0155$ & -4 \\
\hline Iron (Fe) & ICP-OES & T189 & 14 & 0.032 & 0.006 & 18 & $0.0325 \pm 0.00408$ & -2 \\
\hline
\end{tabular}


Table 14. Measurement of concentrations of dissolved constituents in standard reference water samplesContinued.

\begin{tabular}{|c|c|c|c|c|c|c|c|c|}
\hline Analyte & $\begin{array}{c}\text { Analytical } \\
\text { Method }\end{array}$ & \begin{tabular}{|l} 
USGS \\
SRWS \\
\end{tabular} & $\mathbf{n}$ & $\mathrm{mg} / \mathrm{L}^{\mathrm{a}}$ & $\mathbf{s}$ & \begin{tabular}{|c|} 
Percent \\
RSD \\
\end{tabular} & $\begin{array}{c}\text { Most Probable } \\
\text { Value, } \mathrm{mg} / \mathrm{L}\end{array}$ & $\begin{array}{c}\text { Percent } \\
\text { Difference }\end{array}$ \\
\hline Manganese (Mn) & GFAAS & $\mathrm{T} 181$ & 7 & 0.0127 & 0.0003 & 2.2 & $0.0116 \pm 0.000593$ & 9 \\
\hline Manganese (Mn) & GFAAS & T189 & 7 & 0.0069 & 0.0003 & 5.0 & $0.00655 \pm 0.000397$ & 6 \\
\hline Manganese (Mn) & ICP-OES & $\mathrm{T} 179$ & 6 & 0.043 & 0.002 & 4 & $0.0442 \pm 0.00156$ & -2 \\
\hline Manganese (Mn) & ICP-OES & $\mathrm{T} 181$ & 6 & 0.011 & 0.001 & 10 & $0.0116 \pm 0.000593$ & -4 \\
\hline Manganese (Mn) & ICP-OES & $\mathrm{T} 183$ & 13 & 0.051 & 0.003 & 6 & $0.0492 \pm 0.00156$ & 4 \\
\hline Manganese (Mn) & ICP-OES & T189 & 8 & 0.006 & 0.000 & 5 & $0.00655 \pm 0.000397$ & -13 \\
\hline Copper (Cu) & GFAAS & $\mathrm{T} 179$ & 9 & 0.0034 & 0.0003 & 9.8 & $0.00386 \pm 0.000345$ & -11 \\
\hline Copper $(\mathrm{Cu})$ & GFAAS & $\mathrm{T} 181$ & 16 & 0.0081 & 0.0006 & 6.9 & $0.00779 \pm 0.000452$ & 4 \\
\hline Copper $(\mathrm{Cu})$ & GFAAS & T189 & 18 & 0.0027 & 0.0004 & 13.7 & $0.00294 \pm 0.000189$ & -7 \\
\hline Copper (Cu) & ICP-OES & $\mathrm{T} 179$ & 5 & 0.003 & 0.002 & 55 & $0.00386 \pm 0.000345$ & -14 \\
\hline Copper $(\mathrm{Cu})$ & ICP-OES & $\mathrm{T} 181$ & 5 & 0.007 & 0.001 & 16 & $0.00779 \pm 0.000452$ & -10 \\
\hline Copper $(\mathrm{Cu})$ & ICP-OES & T183 & 9 & $<0.003$ & --- & --- & $0.00306 \pm 0.000156$ & --- \\
\hline Copper $(\mathrm{Cu})$ & ICP-OES & T189 & 11 & 0.003 & 0.001 & 32 & $0.00294 \pm 0.000189$ & -4 \\
\hline Zinc (Zn) & ICP-OES & $\mathrm{T} 179$ & 8 & 0.008 & 0.001 & 9 & $0.0085 \pm 0.000738$ & -11 \\
\hline Zinc (Zn) & ICP-OES & $\mathrm{T} 181$ & 9 & 0.009 & 0.001 & 10 & $0.0105 \pm 0.000741$ & -12 \\
\hline Zinc (Zn) & ICP-OES & $\mathrm{T} 183$ & 13 & 0.091 & 0.006 & 7 & $0.0864 \pm 0.00522$ & 5 \\
\hline Zinc (Zn) & ICP-OES & T189 & 8 & 0.007 & 0.001 & 9 & $0.0078 \pm 0.000604$ & -9 \\
\hline Cadmium (Cd) & GFAAS & $\mathrm{T} 179$ & 17 & 0.0045 & 0.0001 & 2.7 & $0.0044 \pm 0.00017$ & 1 \\
\hline Cadmium (Cd) & GFAAS & $\mathrm{T} 183$ & 17 & 0.0055 & 0.0002 & 2.9 & $0.0054 \pm 0.00029$ & 2 \\
\hline Cadmium (Cd) & ICP-OES & $\mathrm{T} 179$ & 7 & 0.004 & 0.000 & 3 & $0.00442 \pm 0.00017$ & -1 \\
\hline Cadmium (Cd) & ICP-OES & $\mathrm{T} 181$ & 6 & 0.002 & 0.000 & 14 & $0.0016 \pm 0.000093$ & -6 \\
\hline Cadmium (Cd) & ICP-OES & T183 & 9 & 0.006 & 0.000 & 6 & $0.00535 \pm 0.000289$ & 3 \\
\hline Cadmium (Cd) & ICP-OES & T189 & 12 & 0.002 & 0.000 & 14 & $0.00155 \pm 0.000067$ & 4 \\
\hline Chromium (Cr) & GFAAS & $\mathrm{T} 179$ & 17 & 0.0046 & 0.0003 & 7.0 & $0.00422 \pm 0.000334$ & 8 \\
\hline Chromium (Cr) & GFAAS & $\mathrm{T} 179$ & 19 & 0.0056 & 0.0005 & 8.3 & $0.00564 \pm 0.000222$ & 0 \\
\hline Chromium (Cr) & ICP-OES & T179 & 7 & 0.004 & 0.001 & 27 & $0.00422 \pm 0.000334$ & -11 \\
\hline Chromium (Cr) & ICP-OES & $\mathrm{T} 181$ & 8 & 0.005 & 0.001 & 27 & $0.00564 \pm 0.000222$ & -10 \\
\hline Chromium (Cr) & ICP-OES & T183 & 9 & $<0.002$ & --- & --- & $0.00211 \pm 0.000208$ & --- \\
\hline Chromium (Cr) & ICP-OES & T189 & 12 & 0.003 & 0.001 & 17 & $0.00325 \pm 0.000241$ & 0 \\
\hline Cobalt (Co) & GFAAS & $\mathrm{T} 179$ & 17 & 0.0011 & 0.0004 & 36.5 & $0.00110 \pm 0.000052$ & -1 \\
\hline Cobalt (Co) & GFAAS & T181 & 19 & 0.0053 & 0.0006 & 11.1 & $0.00550 \pm 0.000267$ & -4 \\
\hline Cobalt (Co) & ICP-OES & T179 & 7 & $<0.007$ & --- & --- & $0.0011 \pm 0.000052$ & --- \\
\hline Cobalt (Co) & ICP-OES & $\mathrm{T} 181$ & 7 & $<0.007$ & --- & --- & $0.0055 \pm 0.000267$ & --- \\
\hline Cobalt (Co) & ICP-OES & $\mathrm{T} 183$ & 9 & $<0.007$ & --- & --- & $0.00394 \pm 0.000222$ & --- \\
\hline Cobalt (Co) & ICP-OES & T189 & 12 & $<0.007$ & --- & --- & $0.0023 \pm 0.000107$ & --- \\
\hline
\end{tabular}


Table 14. Measurement of concentrations of dissolved constituents in standard reference water samplesContinued.

\begin{tabular}{|c|c|c|c|c|c|c|c|c|}
\hline Analyte & $\begin{array}{c}\text { Analytical } \\
\text { Method }\end{array}$ & \begin{tabular}{|l|} 
USGS \\
SRWS \\
\end{tabular} & $\mathbf{n}$ & $\mathrm{mg} / \mathbf{L}^{\mathrm{a}}$ & $\mathbf{s}$ & $\begin{array}{c}\text { Percent } \\
\text { RSD } \\
\end{array}$ & $\begin{array}{c}\text { Most Probable } \\
\text { Value, mg/L }\end{array}$ & $\begin{array}{c}\text { Percent } \\
\text { Difference }\end{array}$ \\
\hline Nickel (Ni) & GFAAS & T163 & 16 & 0.0154 & 0.0010 & 6.5 & $0.0154 \pm 0.00126$ & 0 \\
\hline Nickel (Ni) & GFAAS & T181 & 16 & 0.0045 & 0.0002 & 3.9 & $0.00472 \pm 0.000252$ & -6 \\
\hline Nickel (Ni) & GFAAS & T183 & 15 & 0.0011 & 0.0002 & 14.3 & $0.00124 \pm 0.000185$ & -10 \\
\hline Nickel (Ni) & ICP-OES & T179 & 5 & $<0.002$ & --- & --- & $0.00141 \pm 0.000445$ & --- \\
\hline Nickel (Ni) & ICP-OES & $\mathrm{T} 181$ & 5 & 0.004 & 0.001 & 35 & $0.00472 \pm 0.000252$ & -24 \\
\hline Nickel (Ni) & ICP-OES & T183 & 13 & $<0.002$ & --- & --- & $0.00124 \pm 0.000185$ & --- \\
\hline Nickel (Ni) & ICP-OES & T189 & 16 & $<0.002$ & --- & --- & $0.00073 \pm 0.000441$ & --- \\
\hline Lead (Pb) & GFAAS & T179 & 15 & 0.0017 & 0.0002 & 10.0 & $0.00186 \pm 0.000141$ & -8 \\
\hline Lead $(\mathrm{Pb})$ & GFAAS & T183 & 16 & 0.0058 & 0.0002 & 4.0 & $0.00589 \pm 0.000304$ & -2 \\
\hline Lead (Pb) & ICP-OES & T179 & 8 & $<0.008$ & --- & --- & $0.00186 \pm 0.000141$ & --- \\
\hline Lead (Pb) & ICP-OES & T181 & 7 & 0.009 & 0.003 & 38 & $0.0094 \pm 0.000482$ & -8 \\
\hline Lead $(\mathrm{Pb})$ & ICP-OES & T183 & 13 & $<0.008$ & --- & --- & $0.00589 \pm 0.000304$ & --- \\
\hline Lead $(\mathrm{Pb})$ & ICP-OES & T189 & 16 & $<0.008$ & --- & --- & $0.000117 \pm 0.000027$ & --- \\
\hline Beryllium (Be) & ICP-OES & T179 & 6 & 0.004 & 0.000 & 2 & $0.00374 \pm 0.000185$ & -1 \\
\hline Beryllium (Be) & ICP-OES & T181 & 6 & 0.004 & 0.000 & 8 & $0.0046 \pm 0.000263$ & -6 \\
\hline Beryllium (Be) & ICP-OES & T183 & 13 & 0.0008 & 0.000 & 10 & $0.00082 \pm 0.000043$ & 1 \\
\hline Beryllium (Be) & ICP-OES & T189 & 16 & 0.004 & 0.000 & 4 & $0.00369 \pm 0.000248$ & 0 \\
\hline Vanadium (V) & ICP-OES & T179 & 6 & 0.002 & 0.001 & 42 & $0.00284 \pm 0.000282$ & -25 \\
\hline Vanadium (V) & ICP-OES & T181 & 8 & $<0.005$ & --- & --- & $0.00371 \pm 0.000156$ & --- \\
\hline Vanadium (V) & ICP-OES & T183 & 9 & $<0.005$ & --- & --- & $0.00464 \pm 0.000222$ & --- \\
\hline Vanadium (V) & ICP-OES & T189 & 12 & $<0.005$ & --- & --- & $0.00295 \pm 0.000326$ & --- \\
\hline Molybdenum (Mo) & ICP-OES & T179 & 7 & $<0.007$ & --- & --- & $0.00218 \pm 0.000145$ & --- \\
\hline Molybdenum (Mo) & ICP-OES & T181 & 4 & $<0.007$ & --- & --- & $0.00449 \pm 0.000345$ & --- \\
\hline Molybdenum (Mo) & ICP-OES & T183 & 13 & $<0.007$ & --- & --- & $0.0021 \pm 0.000148$ & --- \\
\hline Molybdenum (Mo) & ICP-OES & T189 & 14 & $<0.007$ & --- & --- & $0.0024 \pm 0.000133$ & --- \\
\hline Antimony (Sb) & HGAAS & AMW4 & 1 & 0.0032 & --- & --- & $0.0277 \pm 0.00089$ & 16 \\
\hline Antimony (Sb) & HGAAS & T163 & 5 & 0.0307 & 0.0018 & 5.9 & $0.0325 \pm 0.00189$ & -5 \\
\hline Antimony (Sb) & ICP-OES & T179 & 4 & $<0.02$ & --- & --- & $0.00366 \pm 0.000189$ & --- \\
\hline Antimony (Sb) & ICP-OES & T181 & 6 & $<0.02$ & --- & --- & $0.00343 \pm 0.000141$ & --- \\
\hline Antimony (Sb) & ICP-OES & T183 & 13 & $<0.02$ & --- & --- & $0.00481 \pm 0.000259$ & --- \\
\hline Antimony (Sb) & ICP-OES & T189 & 16 & $<0.02$ & --- & --- & $0.00036 \pm 0.000028$ & --- \\
\hline Selenium (Se) & GFAAS & T189 & 9 & 0.0026 & 0.0008 & 32.6 & $0.00313 \pm 0.000497$ & -18 \\
\hline Selenium (Se) & ICP-OES & T179 & 2 & $<0.03$ & --- & --- & $0.0013 \pm 0.000267$ & --- \\
\hline Selenium (Se) & ICP-OES & $\mathrm{T} 181$ & 7 & $<0.03$ & --- & --- & $0.0013 \pm 0.000237$ & --- \\
\hline Selenium (Se) & ICP-OES & $\mathrm{T} 183$ & 9 & $<0.03$ & --- & --- & $0.0021 \pm 0.000245$ & --- \\
\hline Selenium (Se) & ICP-OES & T189 & 12 & $<0.03$ & --- & --- & $0.00313 \pm 0.000497$ & --- \\
\hline
\end{tabular}


Table 14. Measurement of concentrations of dissolved constituents in standard reference water samplesContinued.

\begin{tabular}{|c|c|c|c|c|c|c|c|c|}
\hline Analyte & $\begin{array}{c}\text { Analytical } \\
\text { Method }\end{array}$ & \begin{tabular}{|l} 
USGS \\
SRWS \\
\end{tabular} & n & $\mathbf{m g} / \mathbf{L}^{\mathrm{a}}$ & $\mathbf{S}$ & $\begin{array}{c}\text { Percent } \\
\text { RSD } \\
\end{array}$ & $\begin{array}{c}\text { Most Probable } \\
\text { Value, mg/L }\end{array}$ & $\begin{array}{c}\text { Percent } \\
\text { Difference }\end{array}$ \\
\hline Arsenic (As) & ICP-OES & T179 & 11 & $<0.02$ & --- & $\overline{---}$ & $0.0019 \pm 0.000189$ & $\overline{---}$ \\
\hline Arsenic (As) & ICP-OES & T181 & 9 & $<0.02$ & --- & --- & $0.0061 \pm 0.000319$ & --- \\
\hline Arsenic (As) & ICP-OES & T183 & 17 & $<0.02$ & --- & --- & $0.0045 \pm 0.000289$ & --- \\
\hline Arsenic (As) & ICP-OES & T189 & 21 & $<0.02$ & --- & --- & $0.00466 \pm 0.000315$ & --- \\
\hline Mercury $(\mathrm{Hg})^{\mathrm{a}}$ & CVAFS & Hg7 & 151 & 256 & 22 & & $220 \pm 80$ & 16 \\
\hline Mercury $(\mathrm{Hg})^{\mathrm{a}}$ & CVAFS & Hg22 & 69 & 1165 & 51 & & $1240 \pm 130$ & -6 \\
\hline \multicolumn{3}{|c|}{${ }^{a}$ Mercury concentrations are ng/L } & & & & & & \\
\hline
\end{tabular}



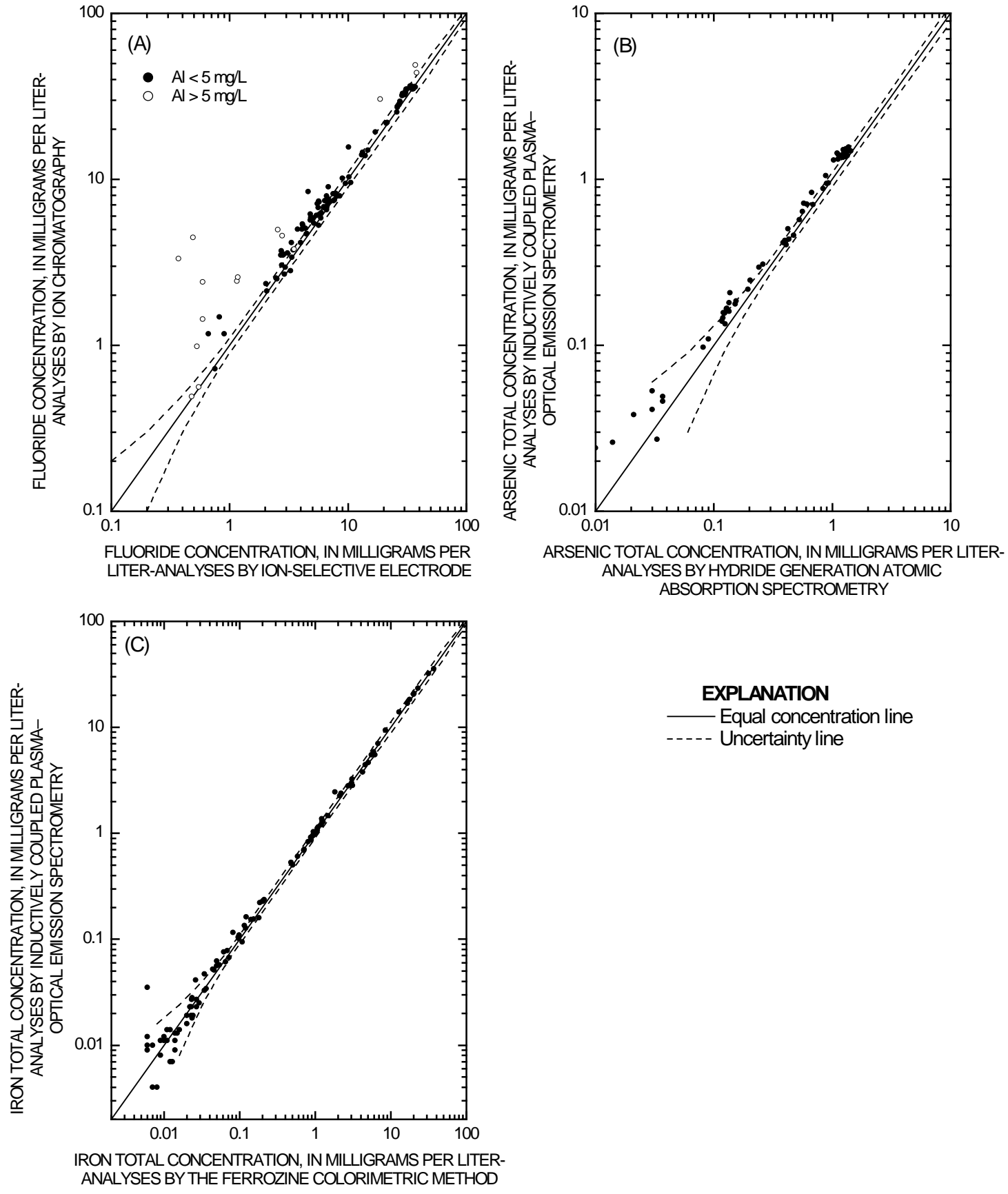

Figure 31. Fluoride (A), total dissolved arsenic (B), and total dissolved iron (C) concentrations determined by alternative methods. 


\section{Acknowledgments}

We extend our appreciation to the staff of Yellowstone National Park for permission to collect water samples and for their assistance on numerous occasions. In particular, we extend our thanks to Hank Haesler and Christie Hendrix of the Yellowstone Center for Natural Resources. We are deeply grateful to all of the Ranger staff at Norris Geyser Basin for escorting us and educating us about the many thermal features of Norris. We are grateful to Maria Perreira, Maria Pilar, Randall Chiu, and Nuno Durães for valuable assistance with field sampling. We also thank Kate Campbell and Kenna Butler of the USGS for many helpful review comments.

\section{References Cited}

Aiken, G.R., 1992, Chloride interference in the analysis of dissolved organic carbon by the wet oxidation method: Environmental Science and Technology, v. 26, p. 2435-2439.

American Public Health Association (APHA), 1998, Method 4500-S ${ }^{2-}$ G. Potentiometric method for sulfide, in Standard methods for the examination of water and wastewater (20th edition): Washington D.C., American Public Health Association, http://standardmethods.org/.

American Public Health Association (APHA), 1985, Method 428C. Methylene blue method for sulfide, in Standard methods for the examination of water and wastewater (14th edition): American Public Health Association, p. 403-405.

American Public Health Association (APHA), 1971, Method 218B. Dissolved oxygen - azide modification, in Standard methods for the examination of water and wastewater (13th edition): American Public Health Association, p. 477-482.

Ball, J.W., McCleskey, R.B., Nordstrom, D.K., and Holloway, J.M., 2006, Water-chemistry data for selected springs, geysers, and streams in Yellowstone National Park, Wyoming, 2003-2005: U.S. Geological Survey Open-File Report 2006-1339, 136 p.

Ball, J.W., McCleskey, R.B., Nordstrom, D.K., Holloway, J.M., and Verplanck, P.L., 2002, Waterchemistry data for selected springs, geysers, and streams in Yellowstone National Park, Wyoming, 1999-2000: U.S. Geological Survey Open-File Report 02-382, 108 p.

Ball, J.W., and Nordstrom, D.K., 1991, User's manual for WATEQ4F, with revised thermodynamic data base and test cases for calculating speciation of major, trace, and redox elements in natural waters: U.S. Geological Survey Open-File Report 91-183, 189 p.

Ball, J.W., Nordstrom, D.K., Jenne, E.A., and Vivit, D.V., 1998a, Chemical analyses of hot springs, pools, geysers, and surface waters from Yellowstone National Park, Wyoming, and vicinity, 1974-1975: U.S. Geological Survey Open-File Report 98-182, 45 p.

Ball, J.W., Nordstrom, D.K., Cunningham, K.M., Schoonen, M.A.A., Xu, Y., and DeMonge, J.M., 1998b, Water-chemistry and on $\llbracket$ site sulfur-speciation data for selected springs in Yellowstone National Park, Wyoming, 1994-1995: U.S. Geological Survey Open-File Report 98-574, 35 p.

Ball, J.W., Nordstrom, D.K., McCleskey, R.B., Schoonen, M.A.A., and Xu, Y., 2001, Waterchemistry and on $\square$ site sulfur-speciation data for selected springs in Yellowstone National Park, Wyoming, 1996-1998: U.S. Geological Survey Open-File Report 01-49, 42 p.

Barnard, W.R., and Nordstrom, D.K., 1980, Fluoride in precipitation-I. Methodology with the fluoride-selective electrode: Atmospheric Environment, v. 16, p. 99-103. 
Barringer, J.L., and Johnsson, P.A., 1996, Theoretical considerations and a simple method for measuring alkalinity and acidity in low-pH waters by Gran titration: U.S. Geological Survey Water-Resources Investigations Report 89-4029, 36 p.

Brinton, T.I., Antweiler, R.C., and Taylor, H.E., 1995, Method for the determination of dissolved chloride, nitrate, and sulfate in natural water using ion chromatography: U.S. Geological Survey Open-File Report 95-426A, 16 p.

Boyd, E.S., King, S.A., Tomberlin, J.K., Nordstrom, D.K., Krabbenhoft, D.P., Barkay, T., and Geesey, G.G., 2009, Methylmercury enters an aquatic food web through acidophilic microbial mats in Yellowstone National Park, Wyoming: Environmental Microbiology, v. 11, p. 950-959.

Charlton, S.R., Macklin, C.L., and Parkhurst, D.L., 1997, PHREEQCI--A graphical user interface for the geochemical computer program PHREEQC: U.S. Geological Survey Water-Resources Investigations Report 97-4222, 9p.

Connor, B.F., Currier, J.P., and Woodworth, M.T., 2001, Results of the U.S. Geological Survey's analytical evaluation program for standard reference samples distributed in October 2000: U.S. Geological Survey Open-File Report 01-137, 116 p.

Coplen, T.B., 1994, Reporting of stable hydrogen, carbon, and oxygen isotopic abundances: Pure and Applied Chemistry, v. 66, p. 273-276.

Coplen, T.B., Wildman, J.D., and Chen, J., 1991, Improvements in the gaseous hydrogen-water equilibrium technique for hydrogen isotope ratio analysis: Analytical Chemistry, v. 63, p. 910912.

DeWild, J.F., Olsen, M.L., and Olund, S.D., 2002, Determination of methylmercury by aqueous phase ethylation, followed by gas chromatographic separation with cold vapor atomic fluorescence detection: U.S. Geological Survey Open-File Report 2001-445, 14 p.

Epstein, S. and Mayeda, T., 1953, Variation of ${ }^{18} \mathrm{O}$ content of water from natural sources: Geochimica et Cosmochimica Acta, v. 4, p. 213-224.

Farrar, J.W., 2000, Results of the U.S. Geological Survey's analytical evaluation program for standard reference samples distributed in October 1999: U.S. Geological Survey Open-File Report 00-227, 143 p.

Fishman, M.J., and Friedman, L.C., eds., 1989, Methods for determination of inorganic substances in water and fluvial sediments: Techniques of Water-Resources Investigations of the U.S. Geological Survey, book 5, chapter A1, p. 55-56.

Gibbs, C.R., 1976, Characterization and application of FerroZine iron reagent as a ferrous iron indicator: Analytical Chemistry, v. 48, no. 8, p. 1197-1201.

Gran, G., 1952, Determination of the equivalence point in potentiometric titrations. Part II: The Analyst, v. 77, p. 661-671.

Hach Company, 1992, DR/2000 Spectrophotometer Procedures Manual: Loveland, Colo., Hach Company Publication 44879-00, p. 515-517.

Horvat, M., Bloom, N.S., and Liang, L., 1993, Comparison of distillation with other current isolation methods for the determination of methylmercury compounds in low level environmental samples. Part 1. Sediments: Analytica Chimica Acta, v. 281, p. 135-152. 
Kennedy, V.C., Jenne, E.A., and Burchard, J.M., 1976, Back-flushing filters for field processing of water samples prior to trace-element analyses: U.S. Geological Survey Open-File Report 76-126, $12 \mathrm{p}$.

King, S.A., Behnke, S., Slack, K., Krabbenhoft, D.P., Nordstrom, D.K., Burr, M.D., and Striegl, R.G., 2006, Mercury in water and biomass of microbial communities in hot springs of Yellowstone National Park, USA; Mercury; distribution, transport, and geochemical and microbial transformations from natural and anthropogenic sources: Applied Geochemistry, v. 21, no. 11, p. 1868-1879.

McCleskey, R.B., Nordstrom, D.K., and Ball, J.W., 2003, Metal interferences and their removal prior to the determination of $\mathrm{As}(\mathrm{T})$ and $\mathrm{As}(\mathrm{III})$ in acid mine waters by hydride generation atomic absorption spectrometry: U.S. Geological Survey Water-Resources Investigations Report 034117, $14 \mathrm{p}$.

McCleskey, R.B., Ball, J.W., Nordstrom, D.K., Holloway, J.M., and Taylor, H.E., 2004, Waterchemistry data for selected hot springs, geysers, and streams in Yellowstone National Park, Wyoming, 2001-2002: U.S. Geological Survey Open-File Report 2004-1316, 100 p.

Moses, C.O., Nordstrom, D.K., and Mills, A.L., 1984, Sampling and analysing mixtures of sulphate sulphite, thiosulphate and polythionate: Talanta, v. 31, p. 331-339.

Nordstrom, D.K., 1977, Thermochemical redox equilibria of ZoBell's solution: Geochimica et Cosmochimica Acta, v. 41, p. 1835-1841.

Nordstrom, D.K., and Alpers, C.N., 1999, Geochemistry of acid mine waters, in Plumlee, G.S., and Logsdon, M.J., eds., The environmental geochemistry of mineral deposits: Littleton, Colo., Society of Economic Geologists, Reviews in Economic Geology, v. 6A, p. 133-160.

Nordstrom, D.K., Ball, J.W., and McCleskey, R.B., 2005, Ground water to surface water: chemistry of thermal outflows in Yellowstone National Park, in Inskeep, W.P., and McDermott, T.R., eds., Geothermal biology and geochemistry in Yellowstone National Park: Bozeman, Montana State University Publications, p. 73-94.

Nordstrom, D.K., McCleskey, R.B., and Ball, J.W., 2009, Sulfur geochemistry of hydrothermal waters in Yellowstone National Park, Wyoming, USA. IV. Acid-sulfate waters: Applied Geochemistry, v. 24, p. 191-207.

Nordstrom, D.K., and Munoz, J.L., 1994, Geochemical Thermodynamics: Boston, Blackwell, $493 \mathrm{p}$.

Nordstrom, D.K., Puigdomènech, I., and McNutt, R.H., 1990, Geochemical modeling of waterrock interactions at the Osamu Utsumi mine and Morro do Ferro analogue study sites, Poços de Caldas, Brazil: Swedish Nuclear Fuel and Waste Management Company (SKB) Technical Report 90-23, 33 p.

Olson, M.L., and DeWild, J.F., 1999, Low-level collection techniques and species-specific analytical methods for mercury in water, sediment, and biota: U.S. Geological Survey WaterResources Investigations Report 99-4018-B, p. 191-199.

Parkhurst, D.L., and Appelo, C.A.J., 1999, User's guide to PHREEQC (version 2) - a computer program for speciation, batch-reaction, one-dimensional transport, and inverse geochemical calculations: U.S. Geological Survey Water-Resources Investigations Report 99-4259, 312 p. 
Planer-Friedrich, B., London, J., McCleskey, R.B., Nordstrom, D.K., and Wallschlaeger, D., 2007, Thioarsenates in geothermal waters of Yellowstone National Park; determination, preservation, and geochemical importance: Environmental Science and Technology, v. 41, no. 15, p. 52455251.

Revesz, K., and Coplen, T.B., 2003a, Hydrogen isotope ratio analysis of water by gaseous hydrogen-water equilibration: Standard Operating Procedure (SOP) \#1574, Techniques of the U.S. Geological Survey.

Révész, Kinga, and Coplen, T. B., 2008, Determination of the $\delta\left({ }^{18} \mathrm{O} /{ }^{16} \mathrm{O}\right)$ of Water: RSIL Lab Code 489, in Révész, Kinga, and Coplen, T.B., eds., Methods of the Reston Stable Isotope Laboratory: U.S. Geological Survey Techniques and Methods, book 10, chapt. C2, 28 p.

Roth, D.A., Taylor, H.E., Domagalski, J., Dileanis, P., Peart, D.B., Antweiler, R.C., and Alpers, C.N., 2001, Distribution of inorganic mercury in Sacramento River water and sediments: Archives of Environmental Contamination and Toxicology, v. 40, p. 161-172.

Sherman, L.S., Blum, J.D., Nordstrom, D.K., McCleskey, R.B., Barkay, T., and Vetriani, C., 2009, Mercury isotopic composition of hydrothermal systems in the Yellowstone Plateau volcanic field and Guaymas Basin sea-floor rift: Earth and Planetary Science Letters, v. 279, p. 86-96.

Smith, R.L., Baumgartner, L.K., Miller, D.N., Repert, D.A. and Bohlke, J.K., 2006, Assessment of nitrification potential in ground water using short term, single-well injection experiments: Microbial Ecology, v. 51, p. 22-35.

Stauffer, R.E., Jenne, E.A., and Ball, J.W., 1980. Chemical studies of selected trace elements in hot-spring drainages of Yellowstone National Park: U.S. Geological Survey Professional Paper 1044-F, $20 \mathrm{pp}$.

Stookey, L.L., 1970, Ferrozine-a new spectrophotometric reagent for iron: Analytical Chemistry, v. 42, p. 779-781.

Taylor, H.E., Berghoff, K., Andrews, E.D., Antweiler, R.C., Brinton, T.I., Miller, C., Peart, D.B., and Roth, D.A., 1997, Water quality of springs and seeps in Glen Canyon National Recreation Area: National Park Service Technical Report NPS/NRWRD/NRTR-97/128, 26 p.

To, T.B., Nordstrom, D.K., Cunningham, K.M., Ball, J.W., and McCleskey, R.B., 1999, New method for the direct determination of dissolved Fe(III) concentration in acid mine waters: Environmental Science and Technology, v. 33, no. 5, p. 807-813.

$\mathrm{Xu}, \mathrm{Y}$., and Schoonen, M.A.A., 1995. The stability of thiosulfate in the presence of pyrite in lowtemperature aqueous solutions: Geochimica et Cosmochimica Acta, 59: 4605-4622.

Xu, Y., Schoonen, M.A.A., Nordstrom, D.K., Cunningham, K.M., and Ball, J.W., 1998. Sulfur geochemistry of hydrothermal waters in Yellowstone National Park; I, The origin of thiosulfate in hot spring waters: Geochimica et Cosmochimica Acta, 62(23-24): 3729-3743.

Xu, Y., Schoonen, M.A.A., Nordstrom, D.K., Cunningham, K.M., and Ball, J.W., 2000. Sulfur geochemistry of hydrothermal waters in Yellowstone National Park, Wyoming, USA; II, Formation and decomposition of thiosulfate and polythionate in Cinder Pool: Journal of Volcanology and Geothermal Research, v. 97, p. 407-423.

Xu, Y., Schoonen, M.A.A., and Strongin, D.R., 1996. Thiosulfate oxidation: Catalysis of synthetic spalerited doped with transition metals: Geochimica et Cosmochimica Acta, 60: 4701-4710. 
Whittlesey, L.H., 1988, Wonderland nomenclature: a history of the place names of Yellowstone National Park: Montana Historical Society Press, 867 p.

ZoBell, C.E., 1946, Studies on redox potential of marine sediments: 8. Other methods: Bulletin of the American Association of Petroleum Geologists, v. 30, p. 477-509. 
Appendix-Photographs 


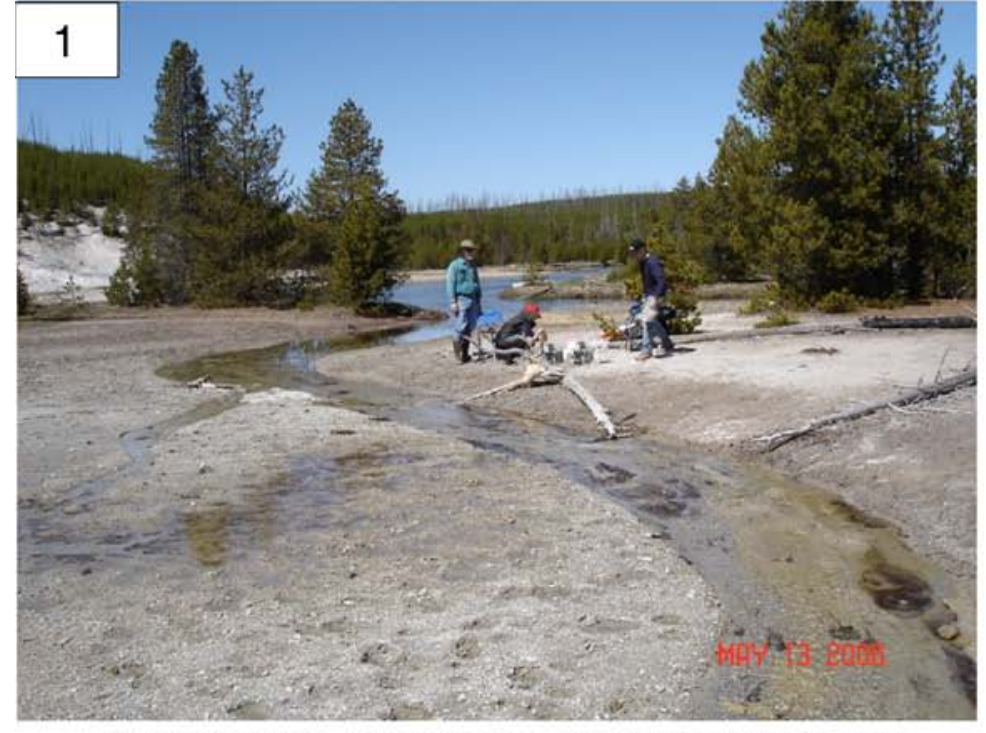

Kirk Nordstrom, Maria Pereira, and Blaine McCleskey

$\infty$

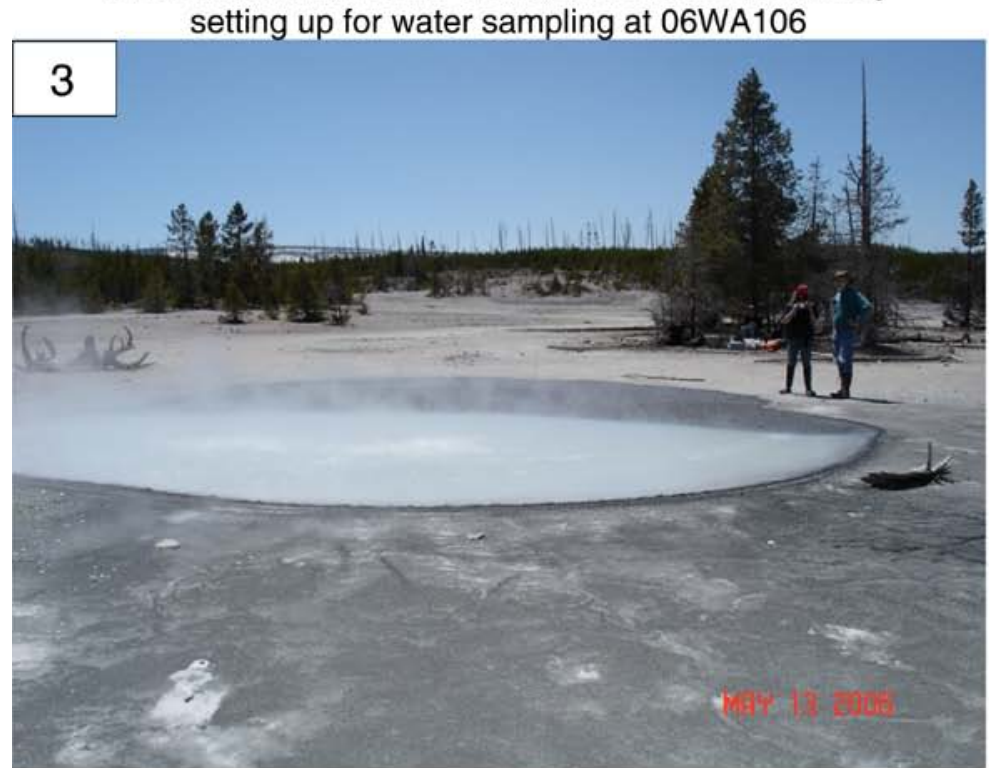

Maria Pereira and Kirk Nordstrom discussing sampling strategies at 06WA108, Cinder Pool

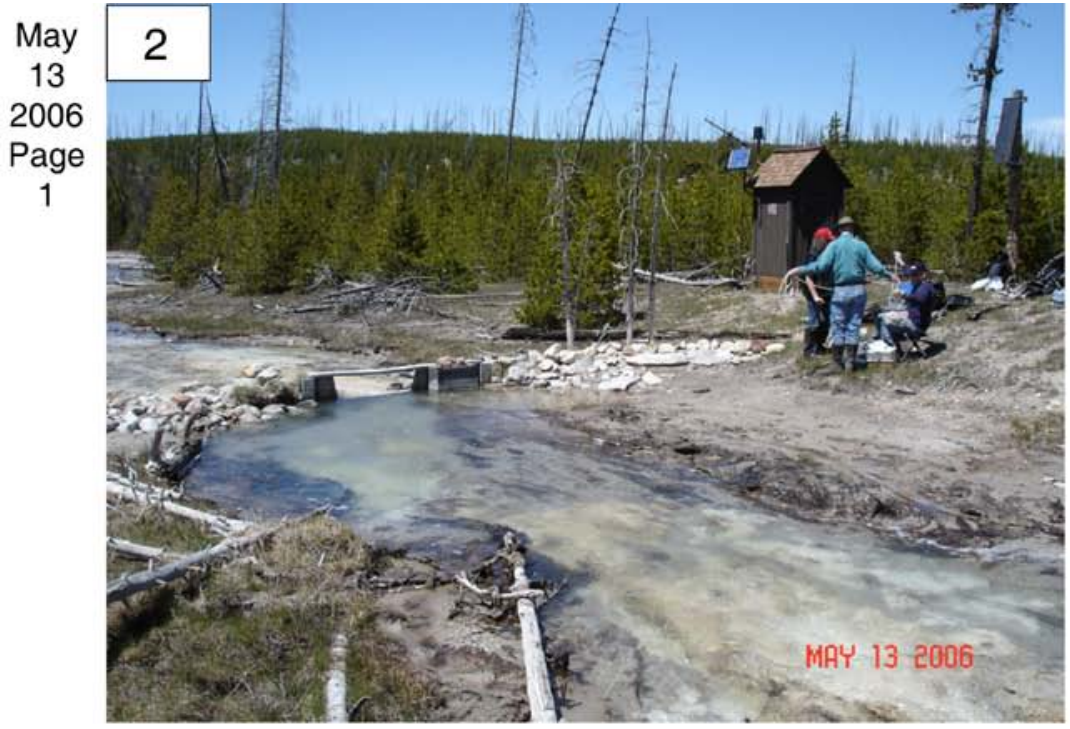

Maria Pereira, Kirk Nordstrom, and Blaine McCleskey setting up for water sampling at 06WA107

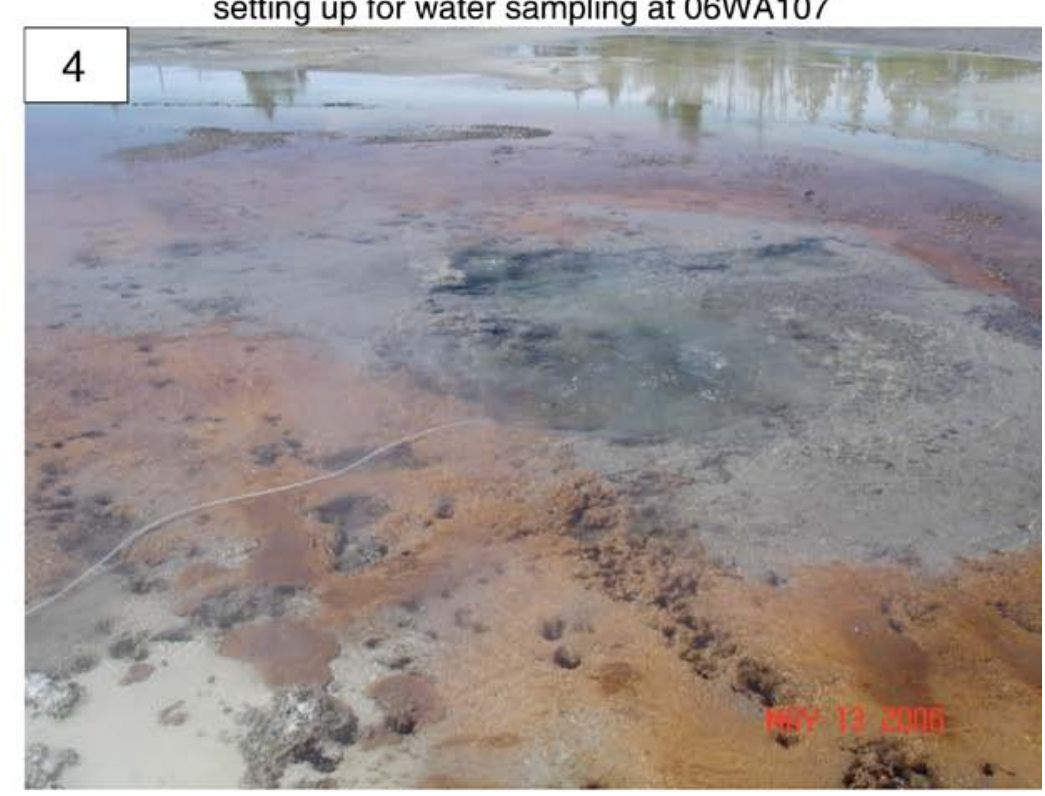

Sampling line in place at 06WA109 

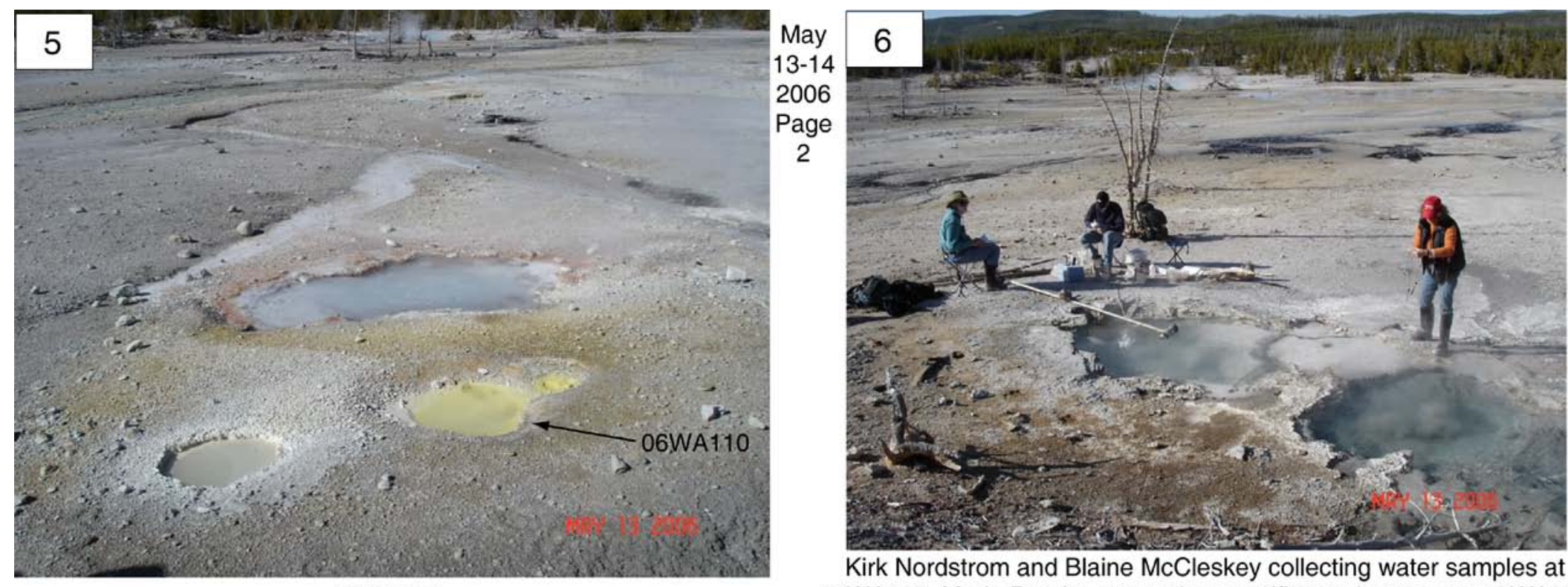

$\stackrel{\infty}{\forall}$

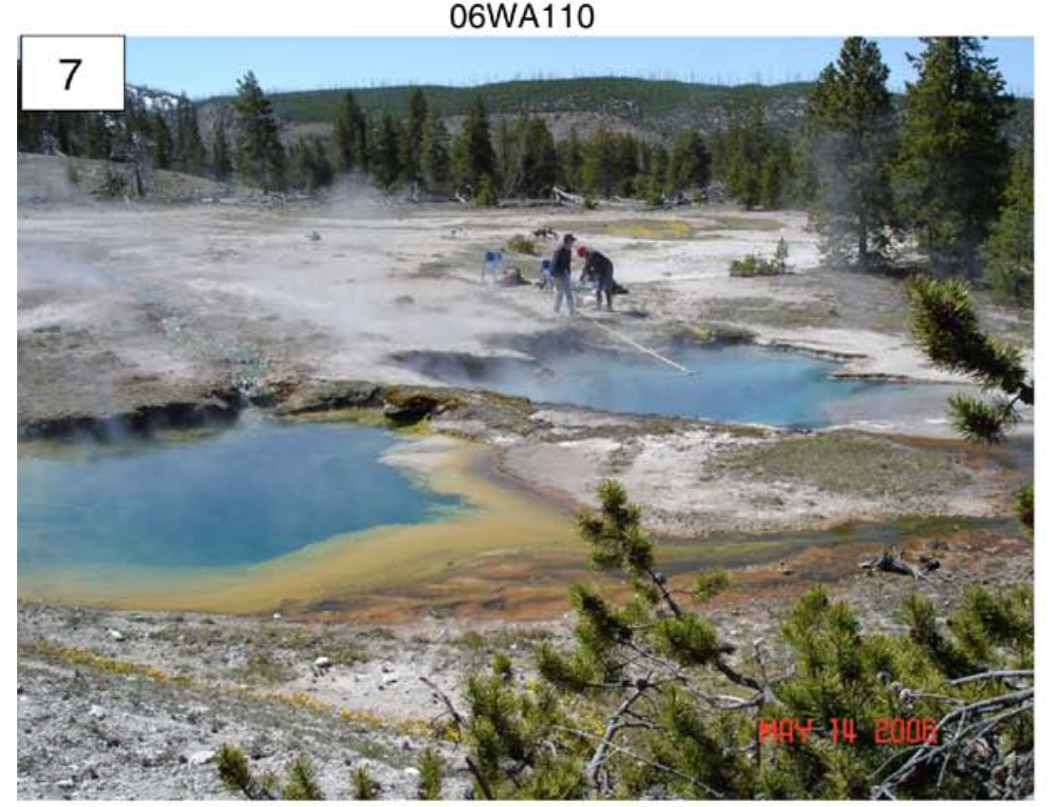

Blaine McCleskey and Maria Pereira water sampling at 06WA113

Kirk Nordstrom and Blaine McCleskey collecting water samples at 06WA111, Maria Pereira measuring specific conductance at 06WA112

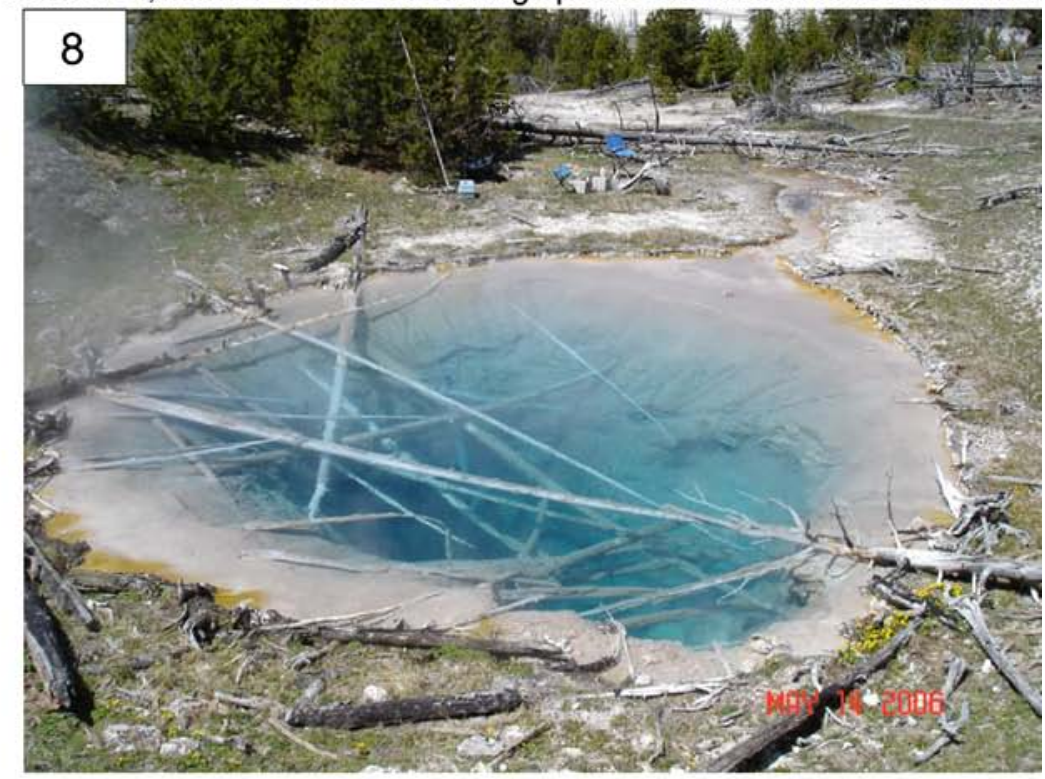

06WA114 


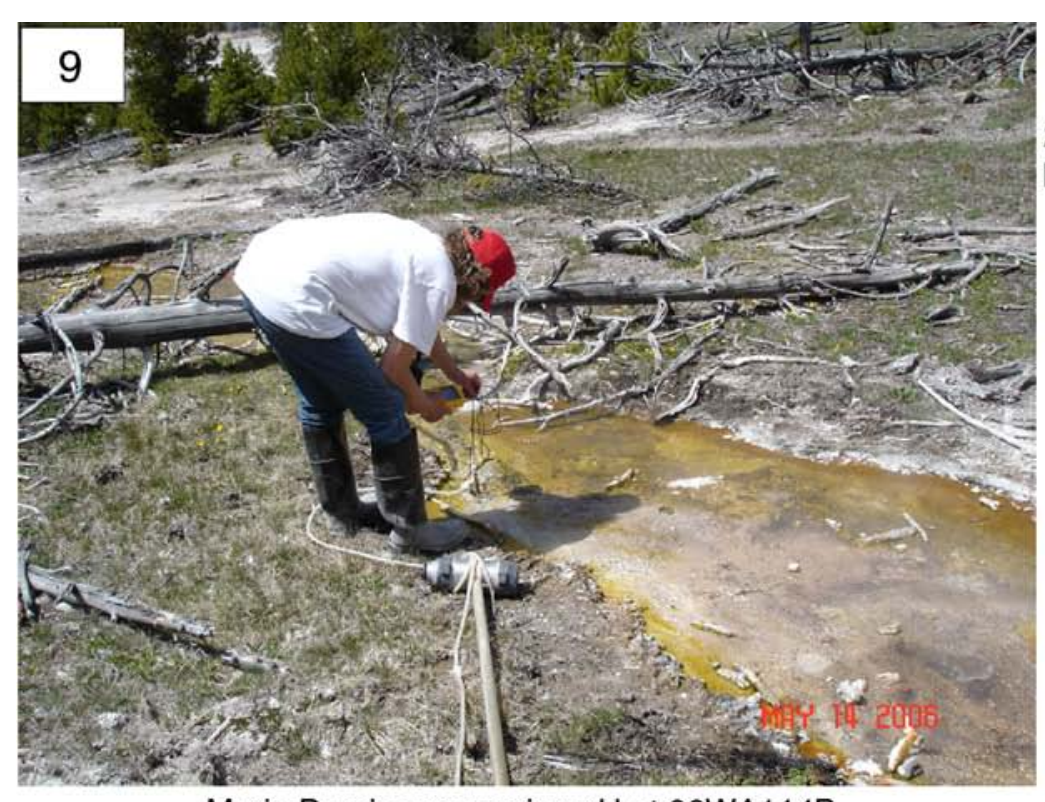

Maria Pereira measuring $\mathrm{pH}$ at 06WA114B

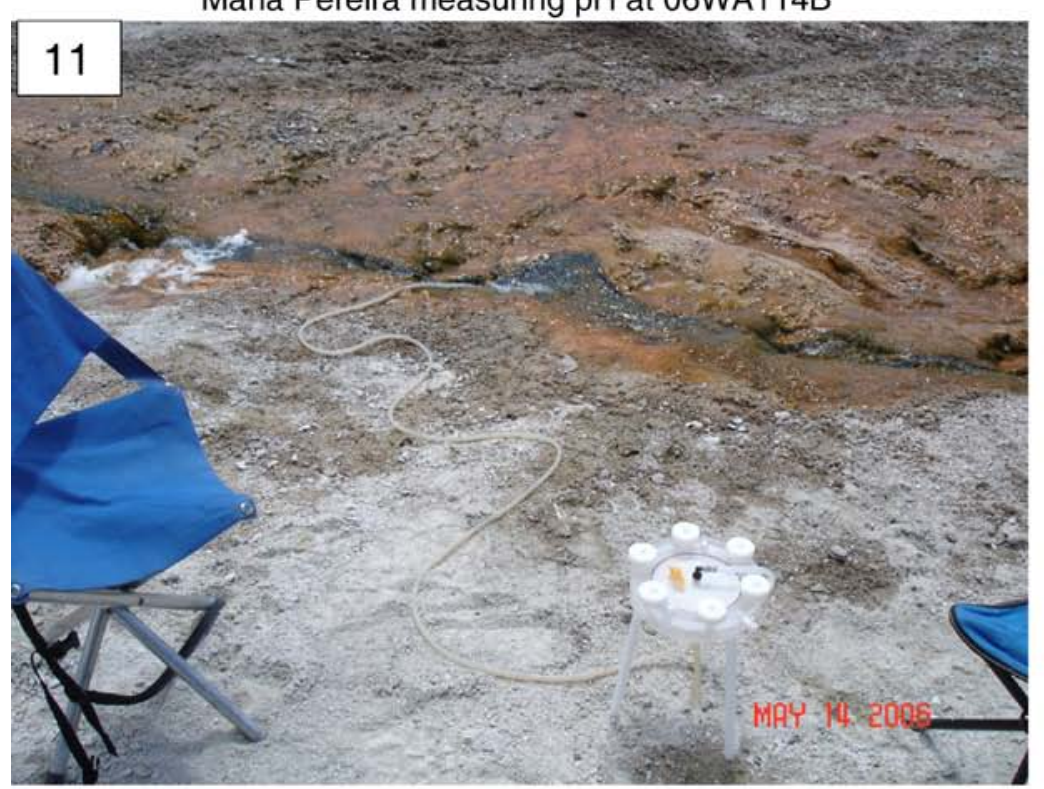

Sampling line and filtration assembly at 06WA114D

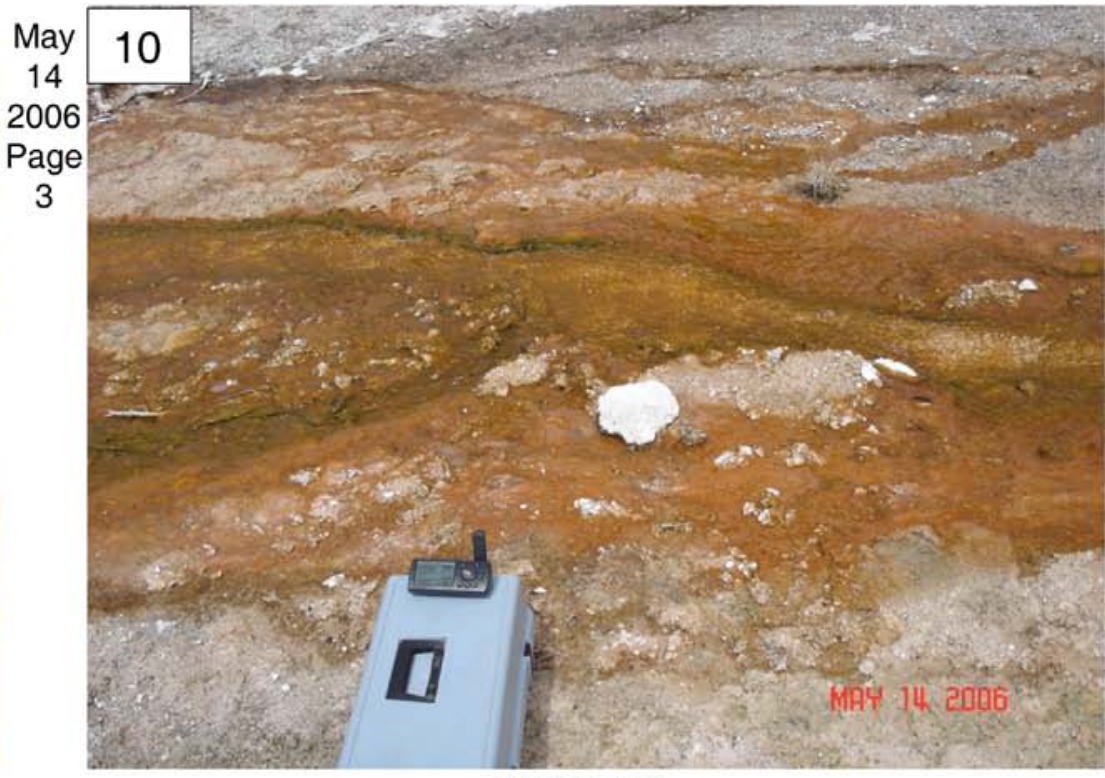

06WA114C

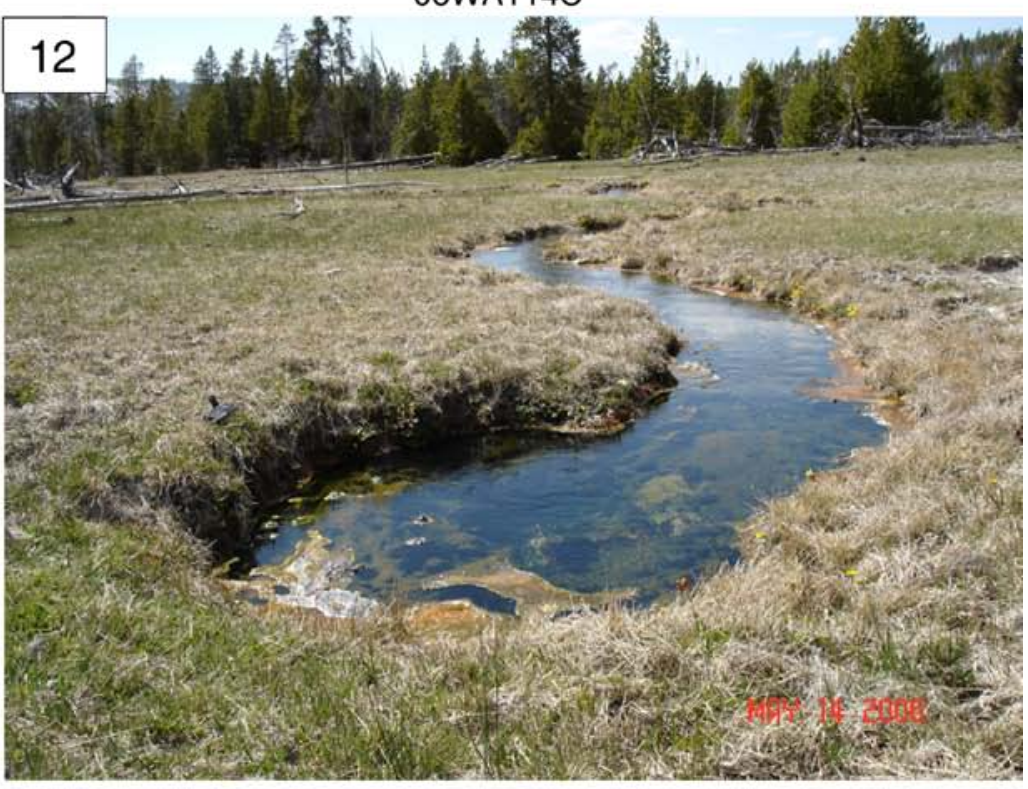

06WA115 


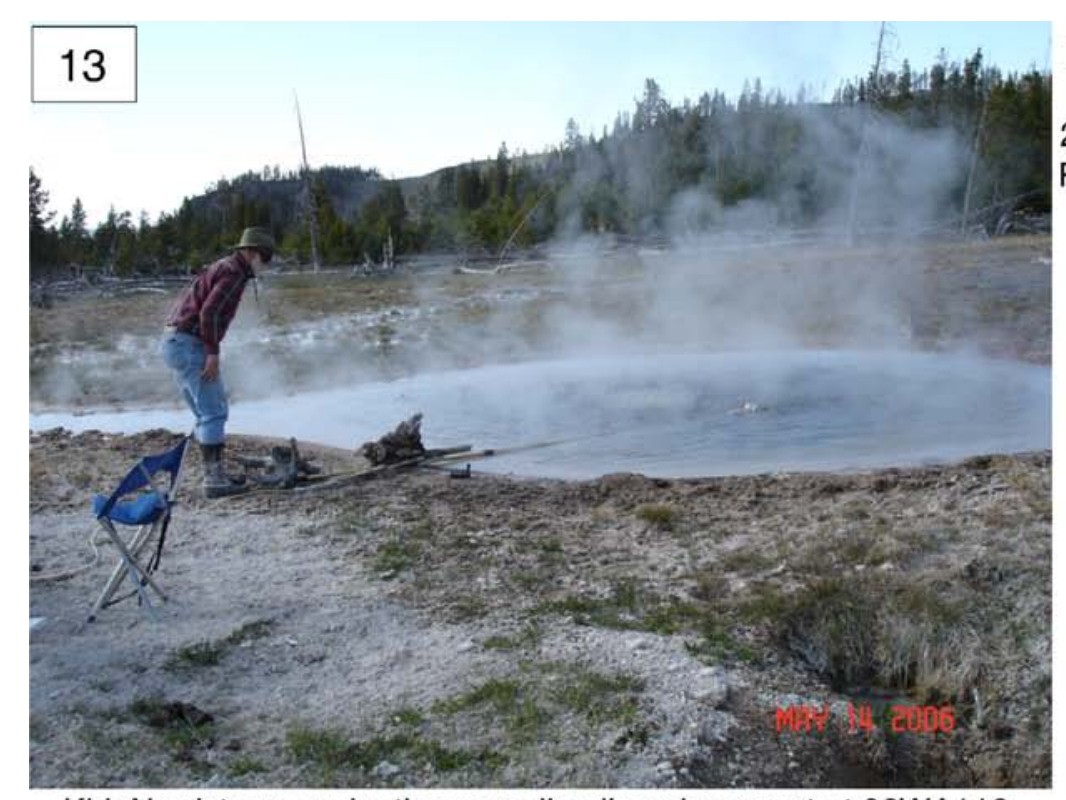

$\infty$

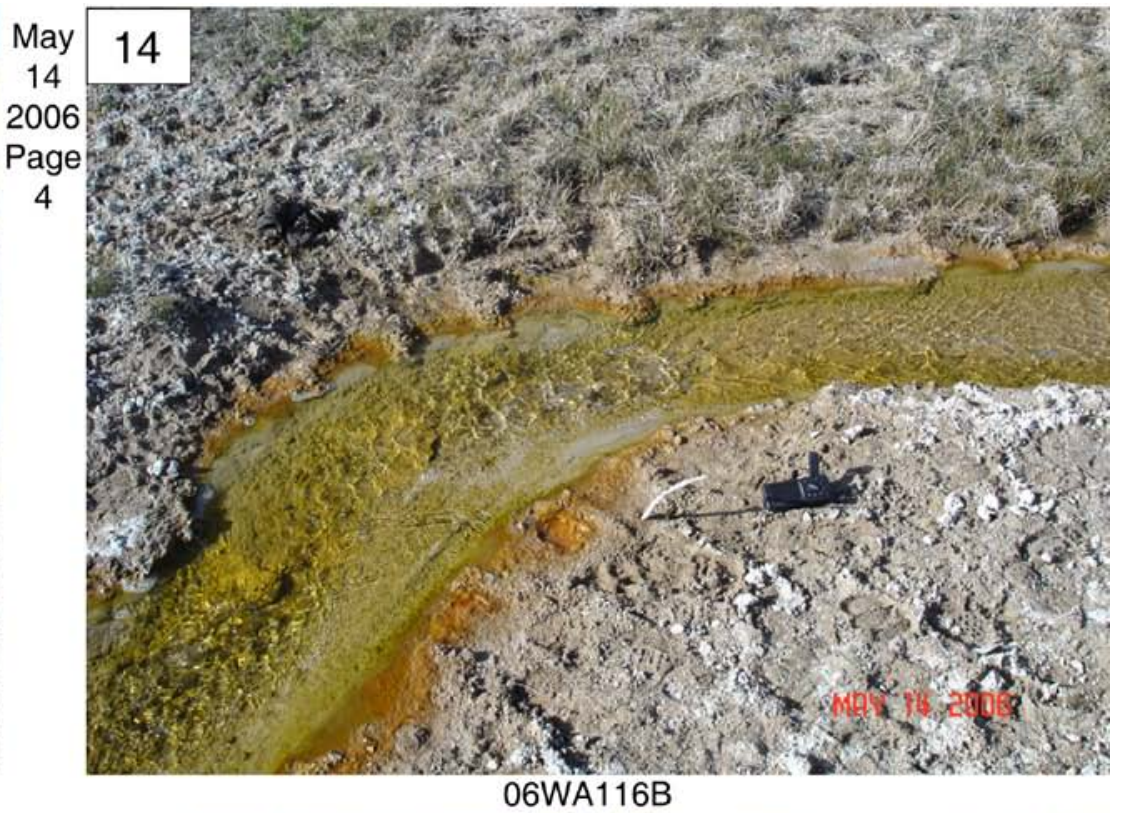
06WA116B

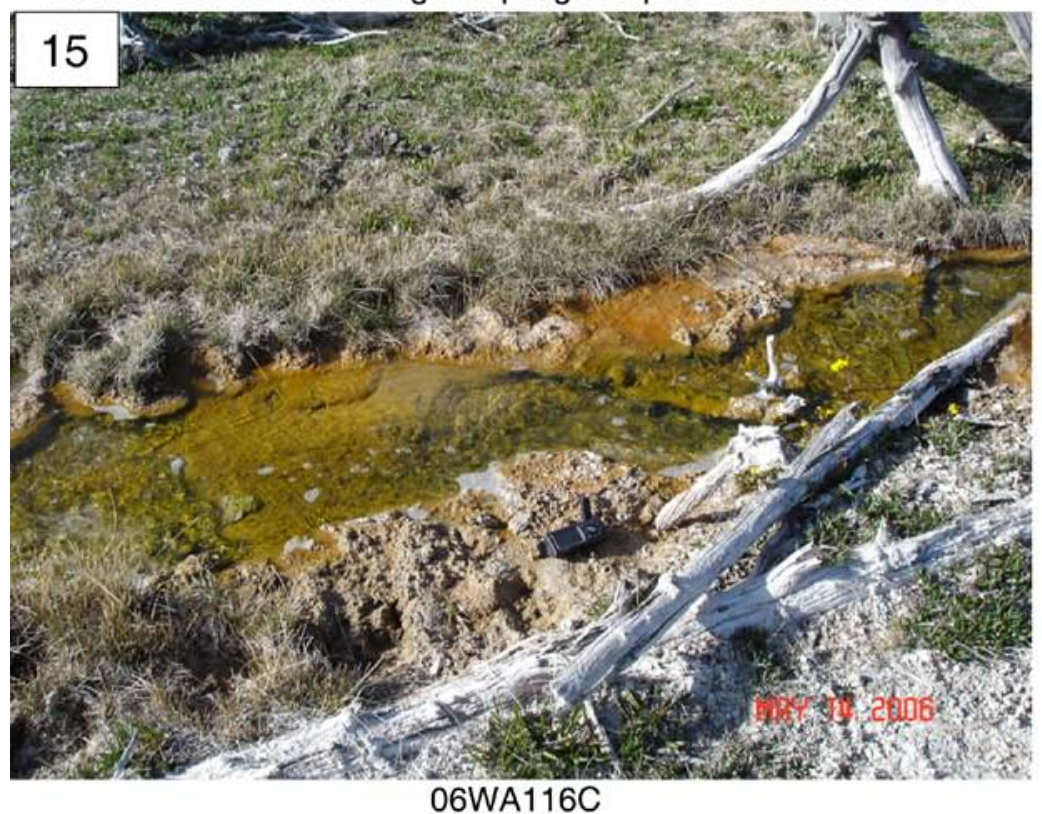

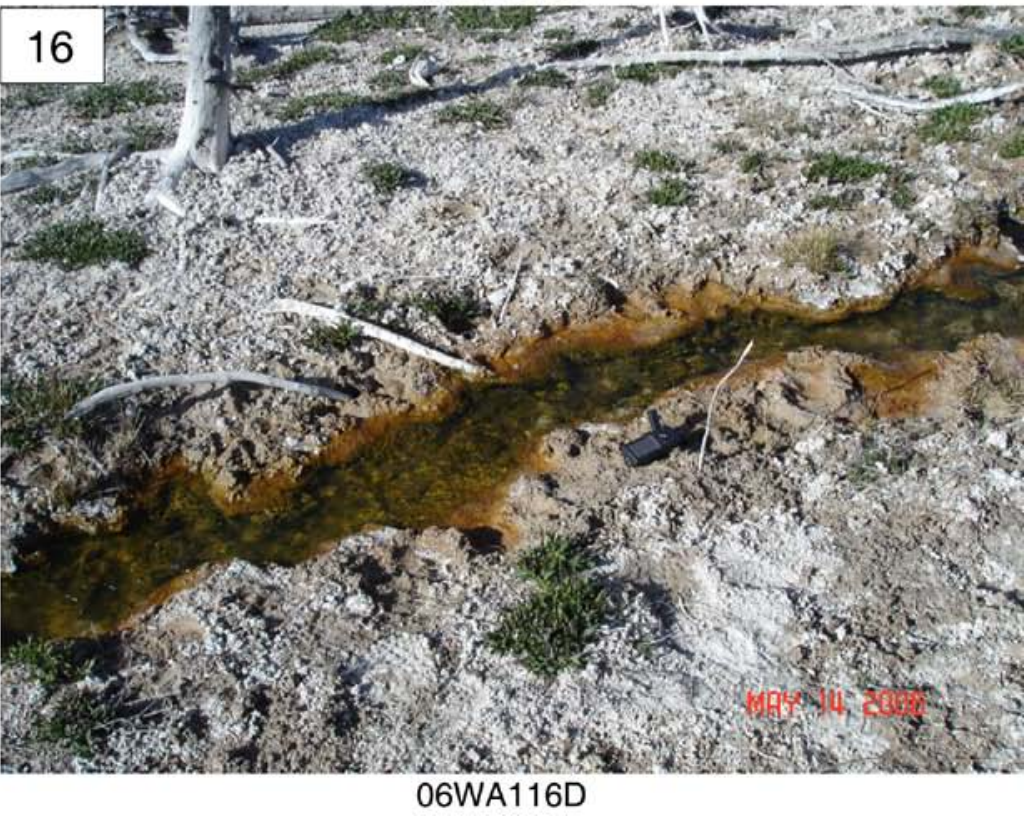



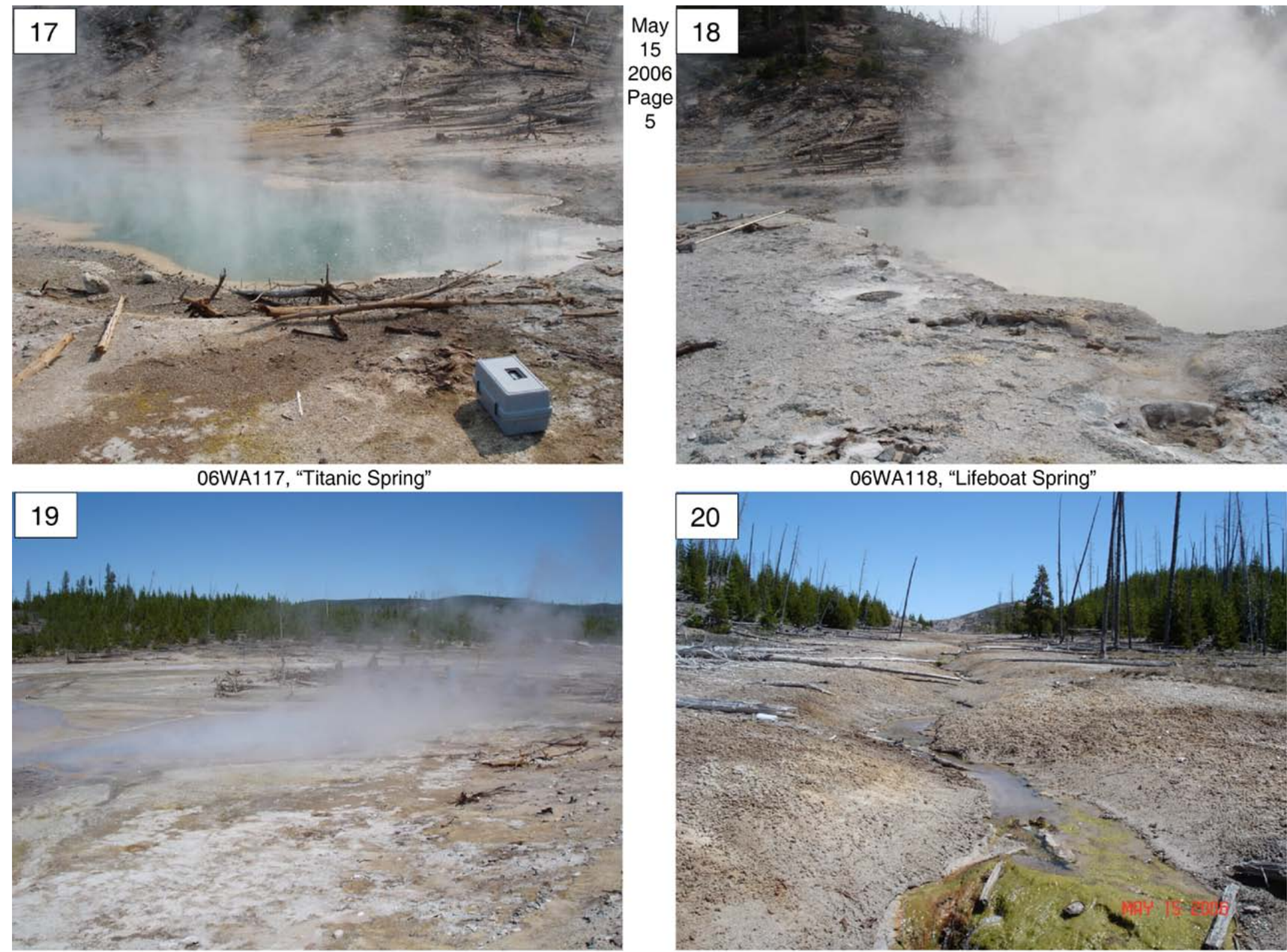

06WA119, "Persnickety Geyser"

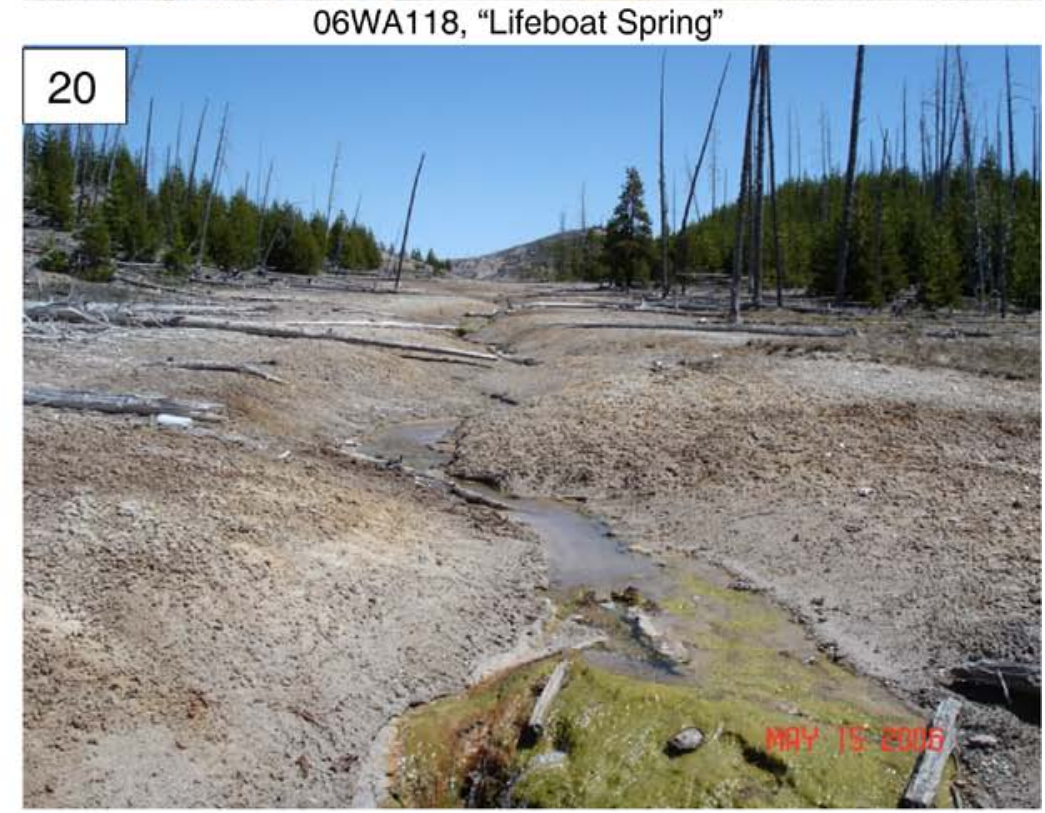

06WA120 

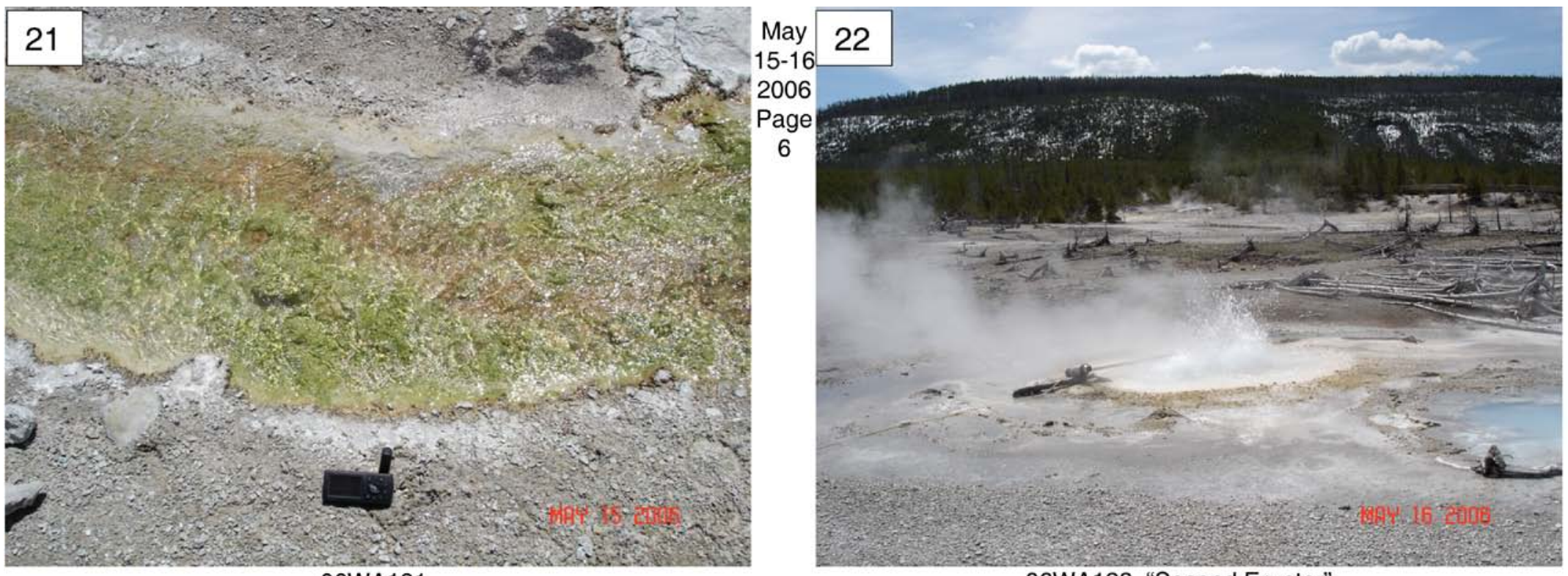

06WA121

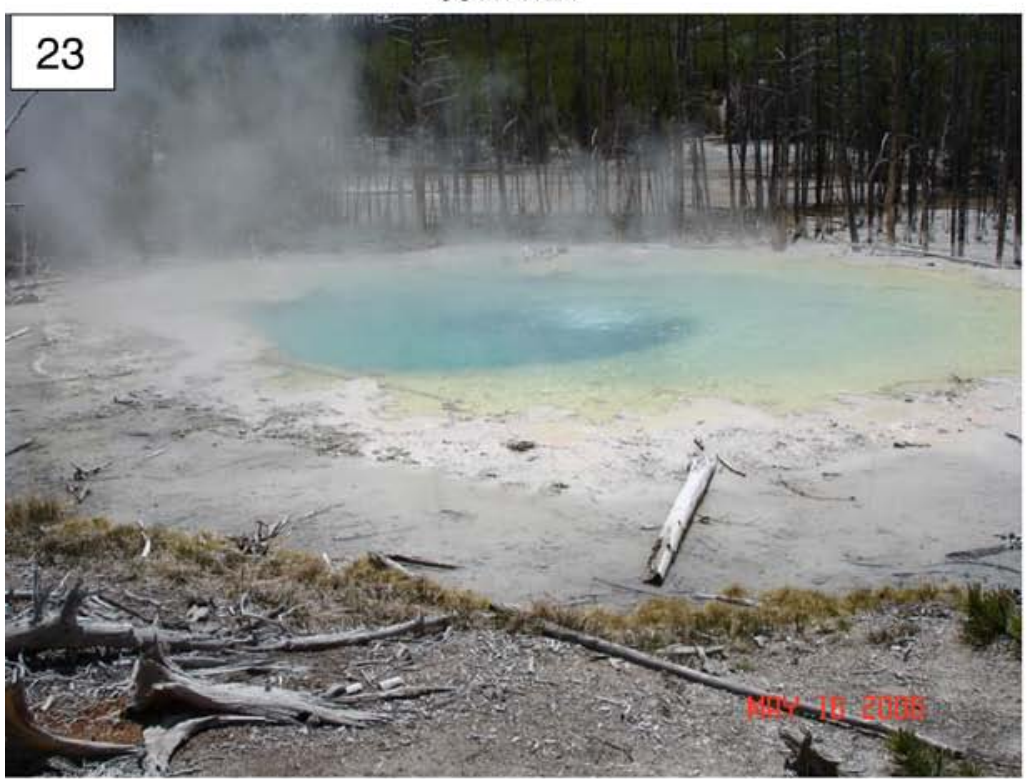

06WA123, Cistern Spring
06WA122, "Second Eruptor"

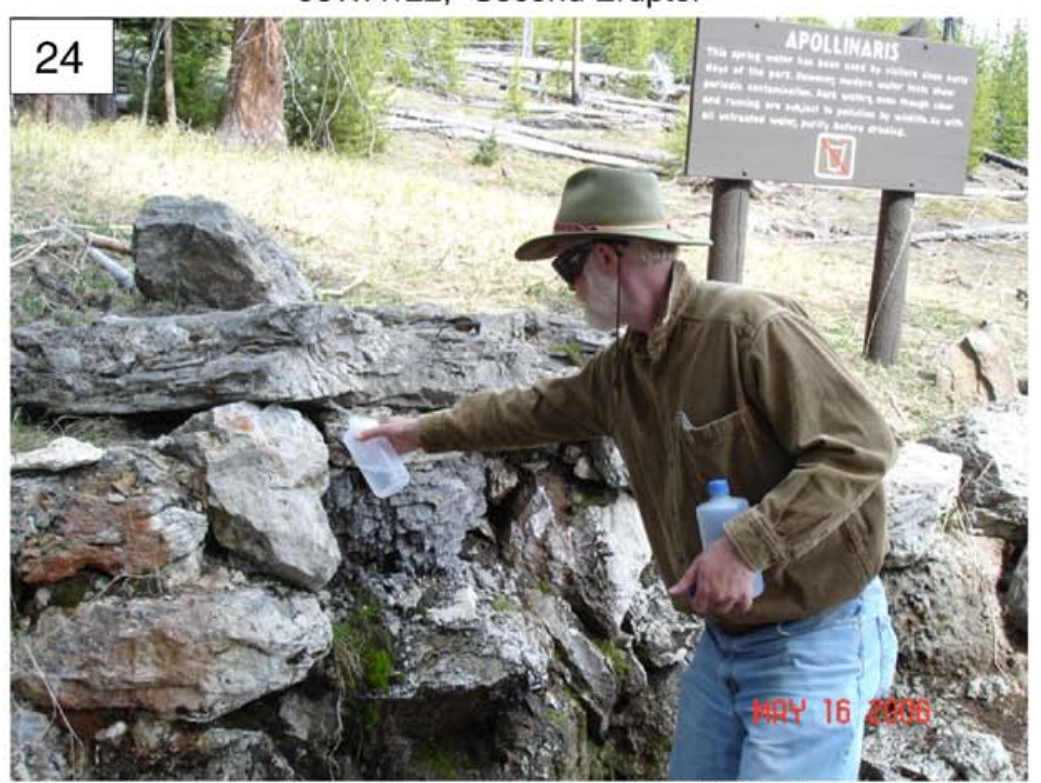

Kirk Nordstrom collecting a water sample at 06WA124, Apollinaris Spring 

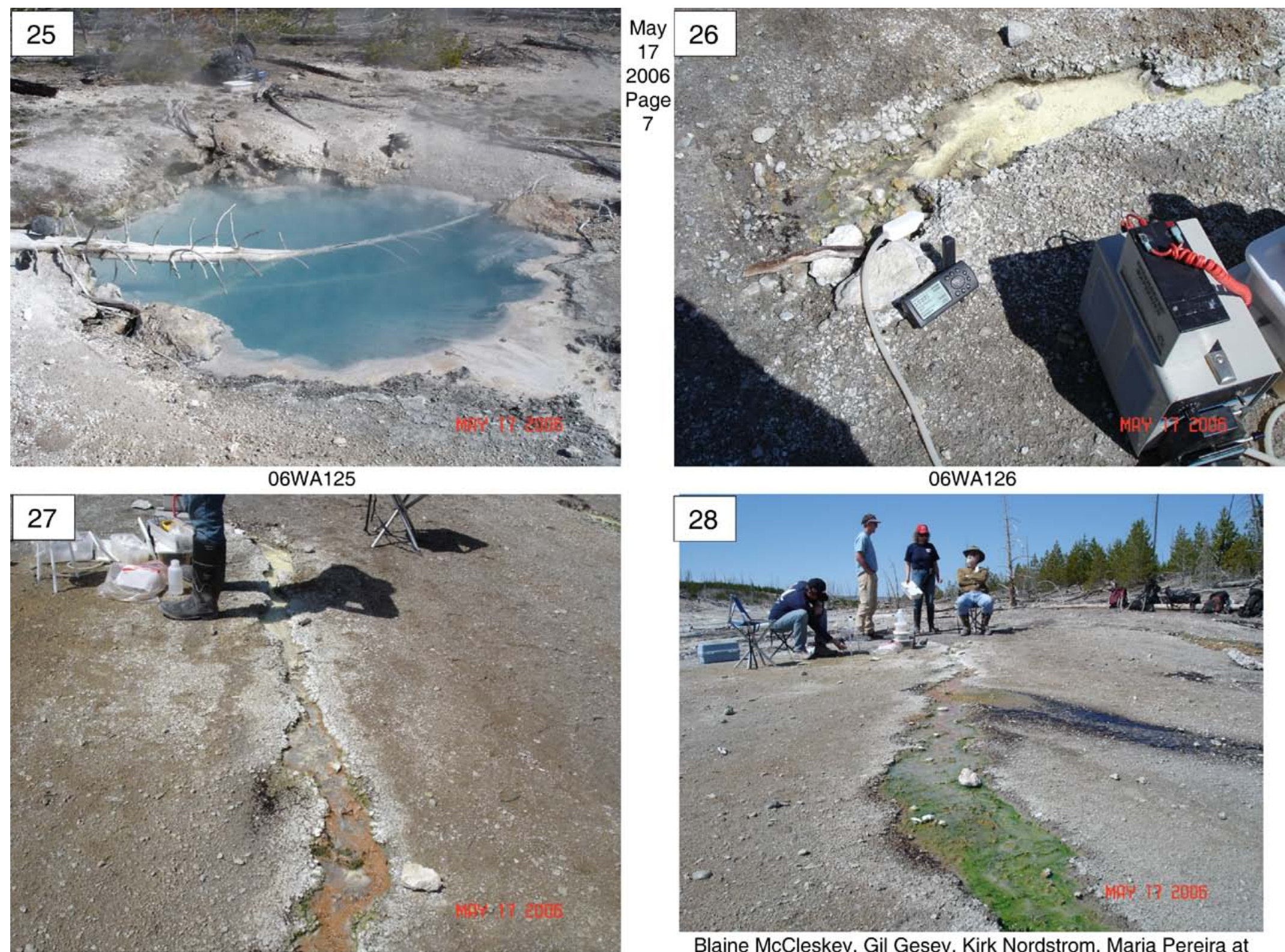

06WA127

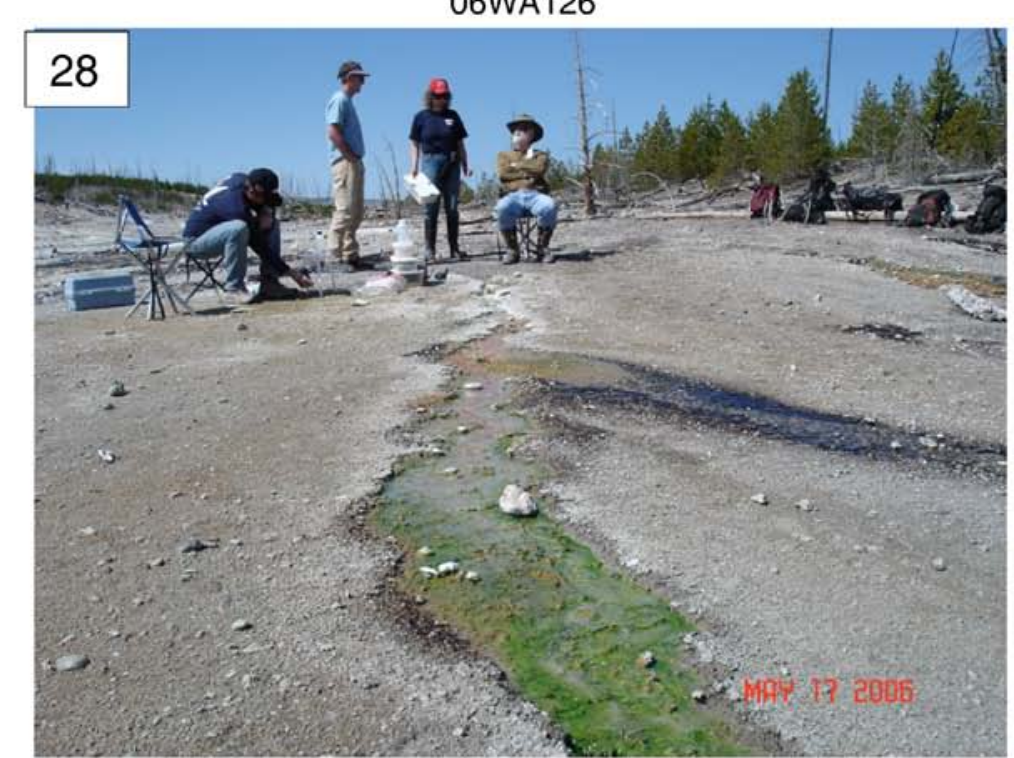

Blaine McCleskey, Gil Gesey, Kirk Nordstrom, Maria Pereira at 06WA126; 06WA128 in foreground 

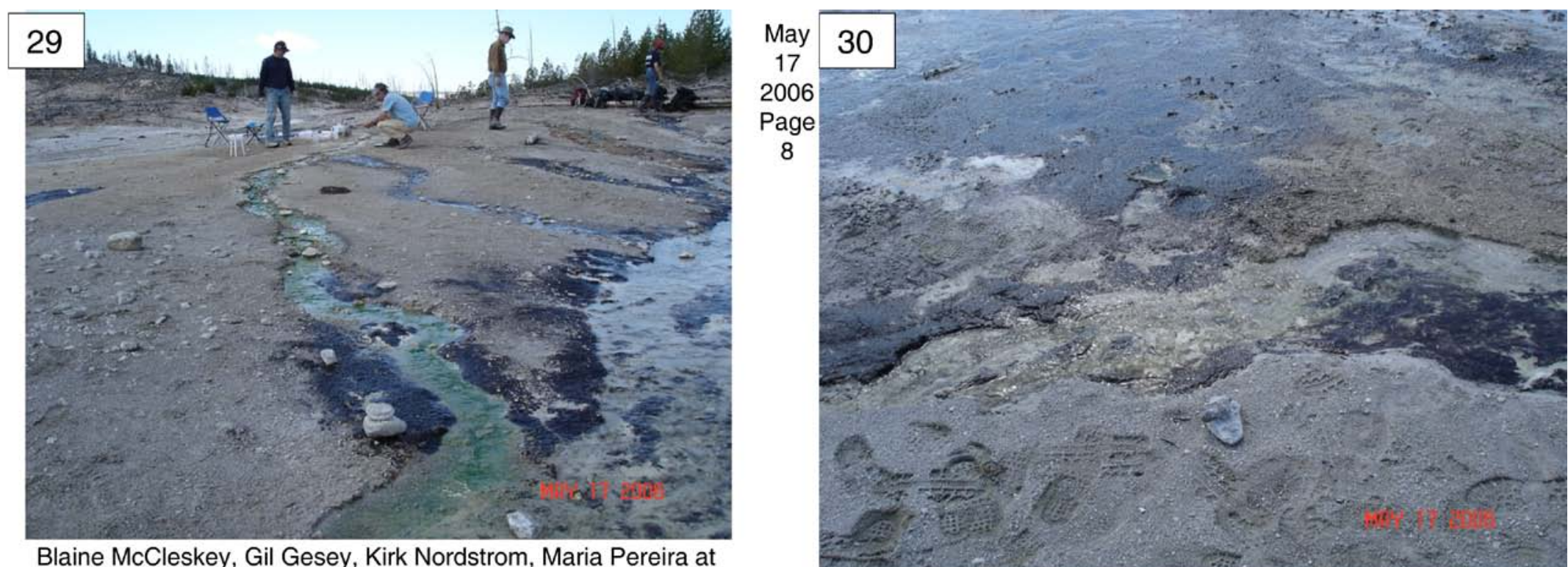

Blaine McCleskey, Gil Gesey, Kirk Nordstrom, Maria Pereira at

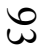
06WA126; 06WA129 and 06WA130 in foreground

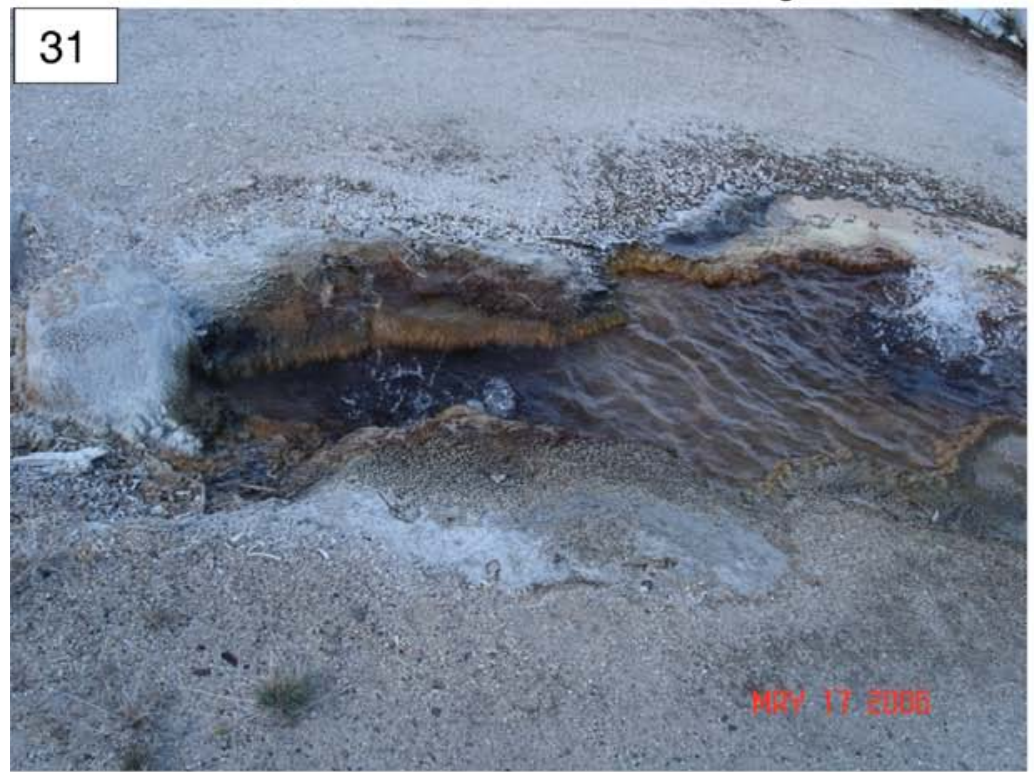

06WA132, Perpetual Spouter

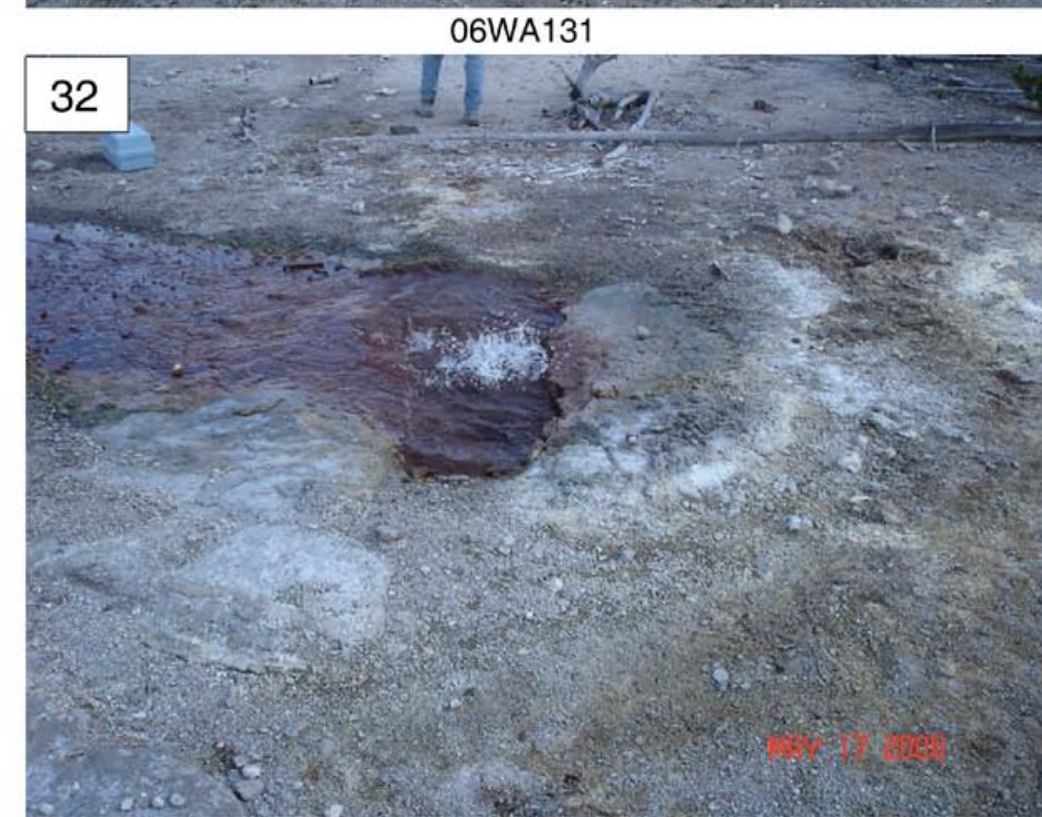

Blaine McCleskey at 06WA133 


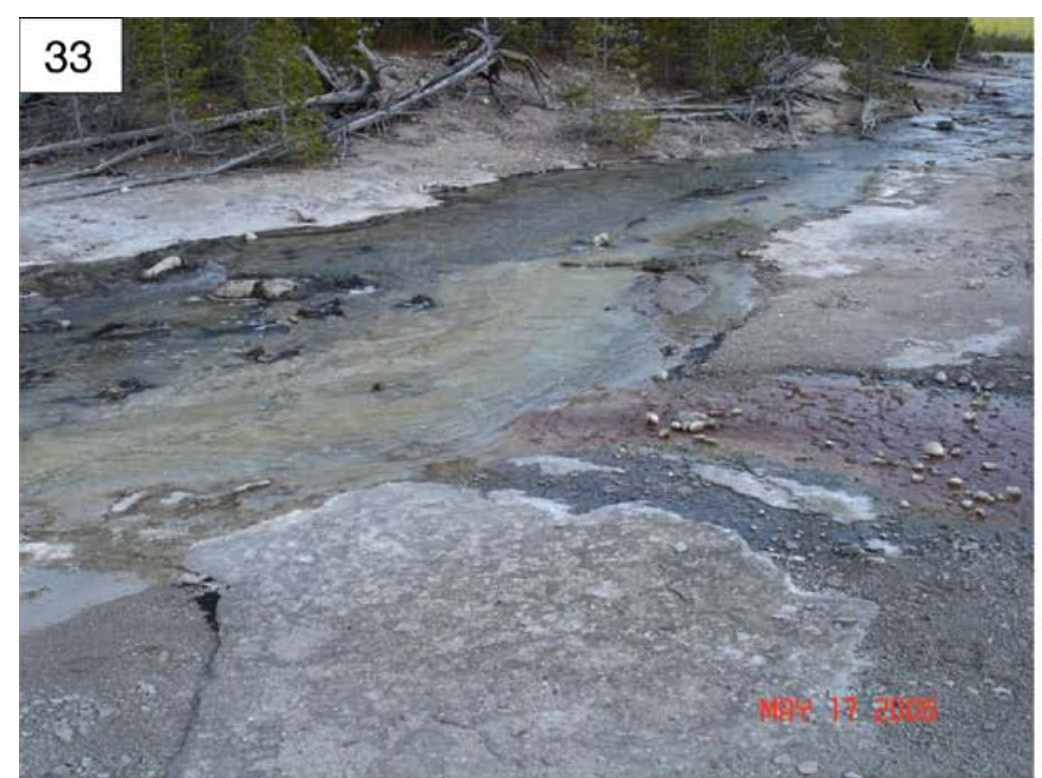

06WA134, Tantalus Creek upstream from Perpetual Spouter

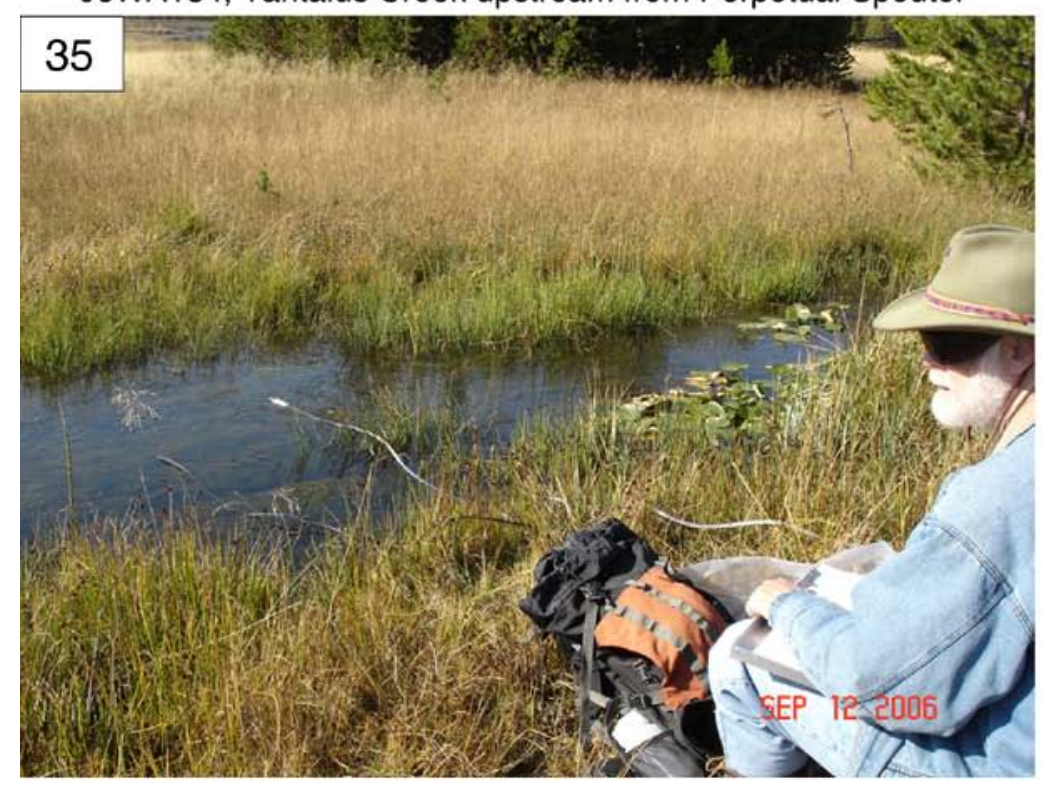

Kirk Nordstrom recording site data at 06WA136, Nymphy Creek

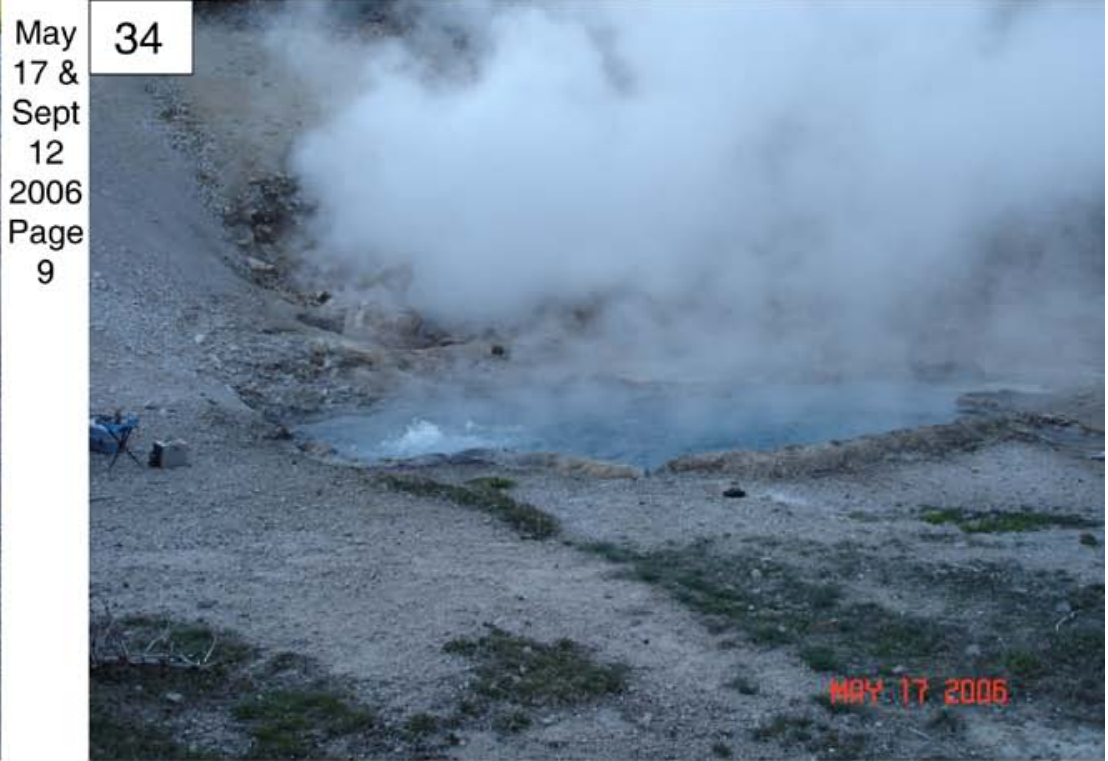

06WA135, Beryl Spring

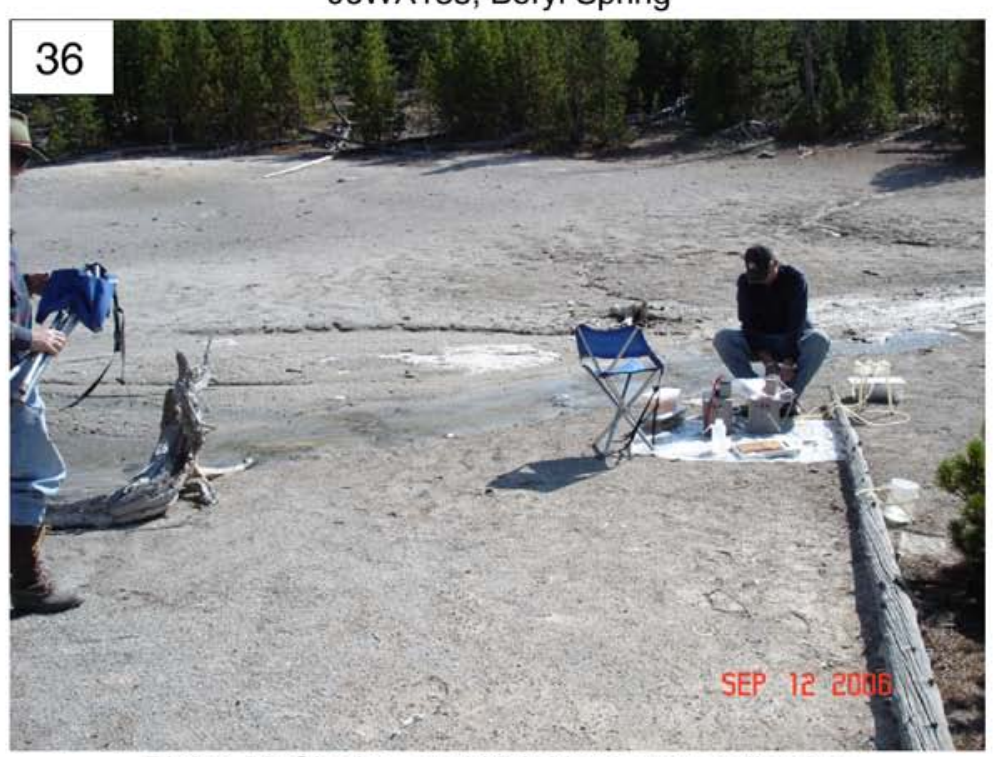

Blaine McCleskey and Kirk Nordstrom collecting water samples at 06WA139 

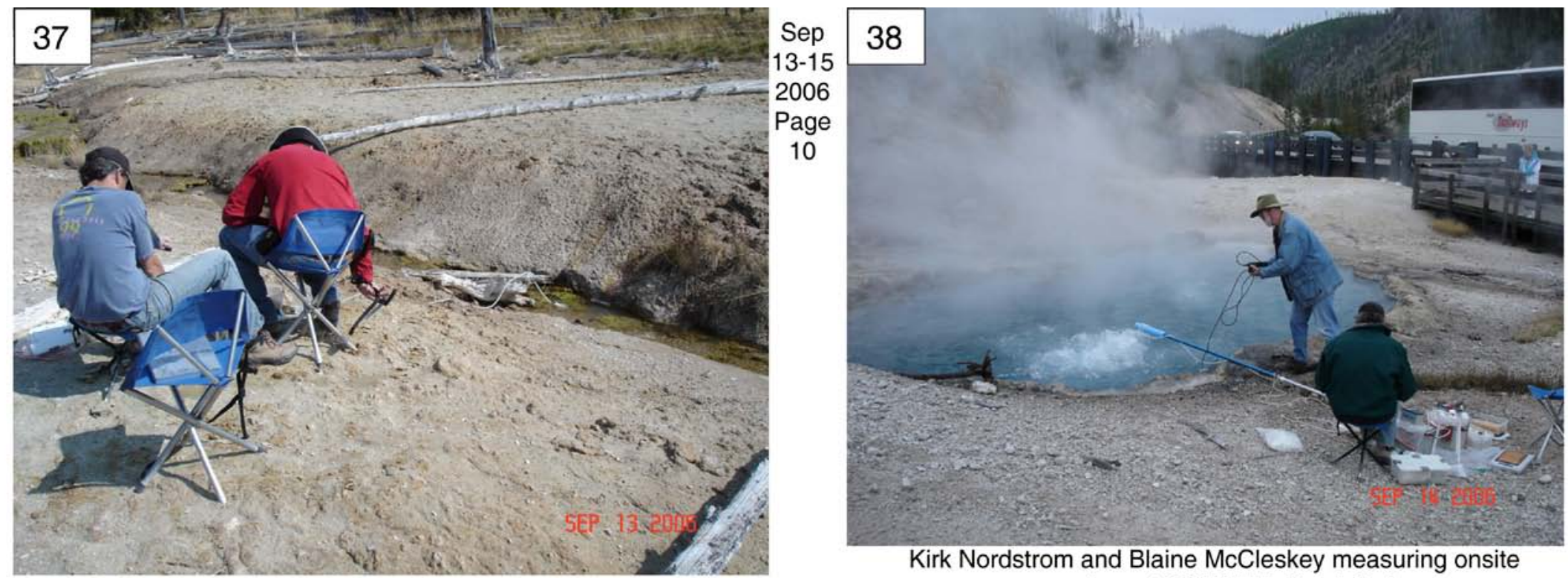

Blaine McCleskey and Jim Ball collecting water samples at 06WA146
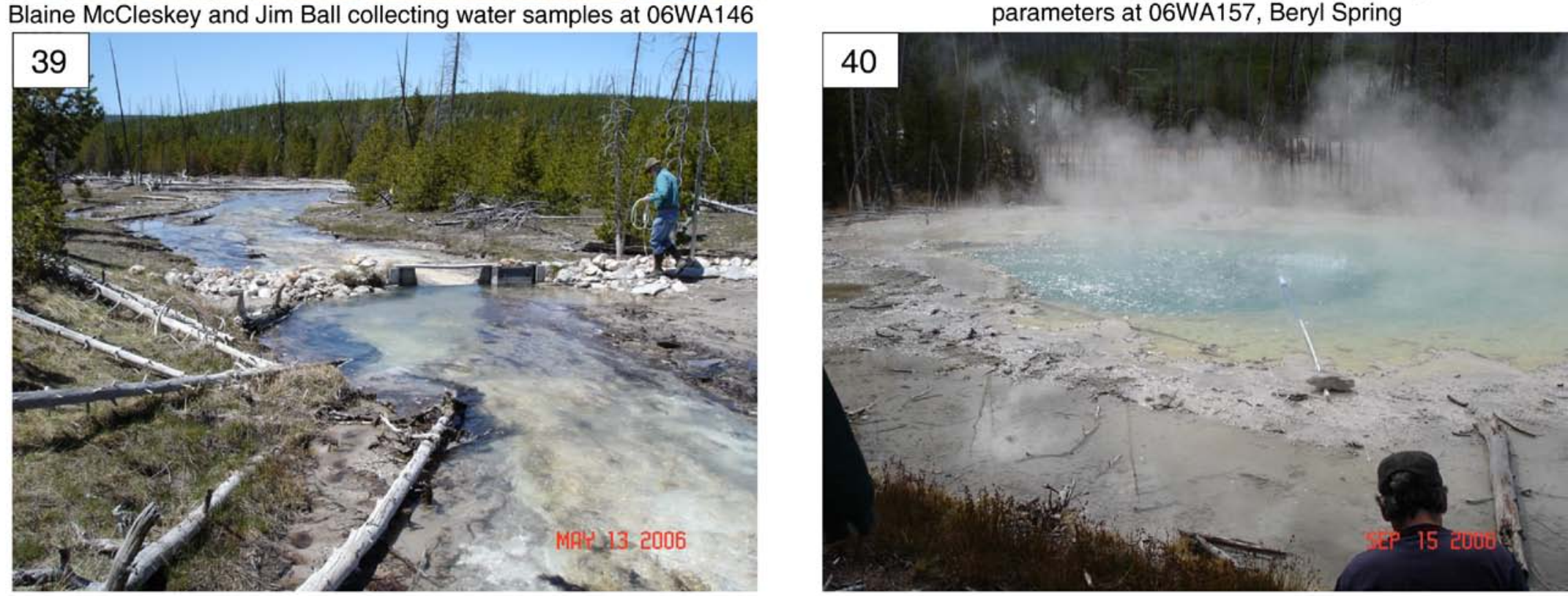

Kirk Nordstrom placing sample line at 06WA158, Tantalus Creek at weir

Blaine McCleskey at 06WA159 Cistern Spring 


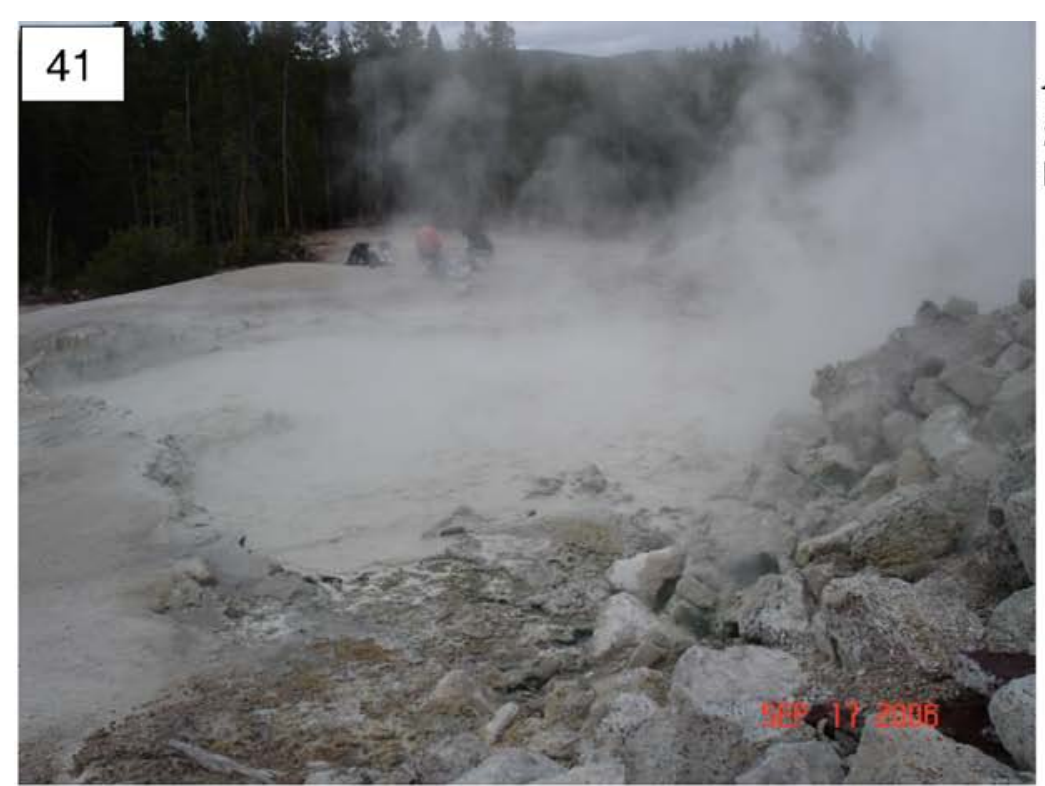

๙
Jim Ball and Joel Blum at 06WA164, Sulphur Caldron

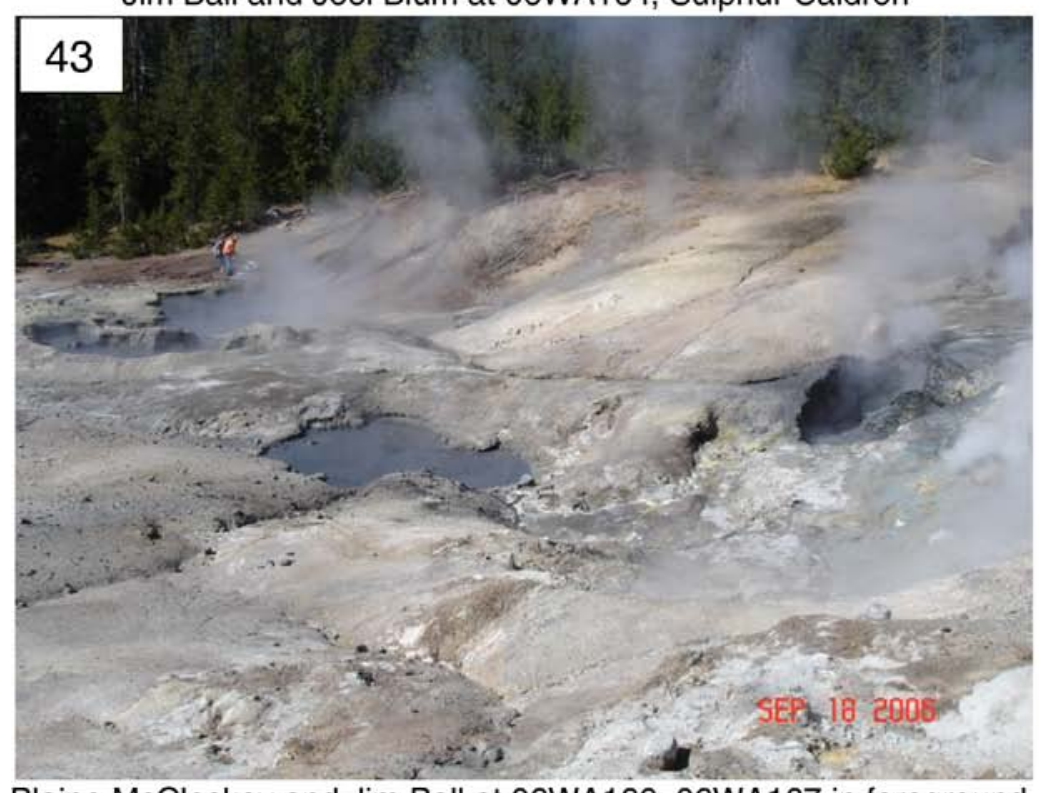

Blaine McCleskey and Jim Ball at 06WA166; 06WA167 in foreground

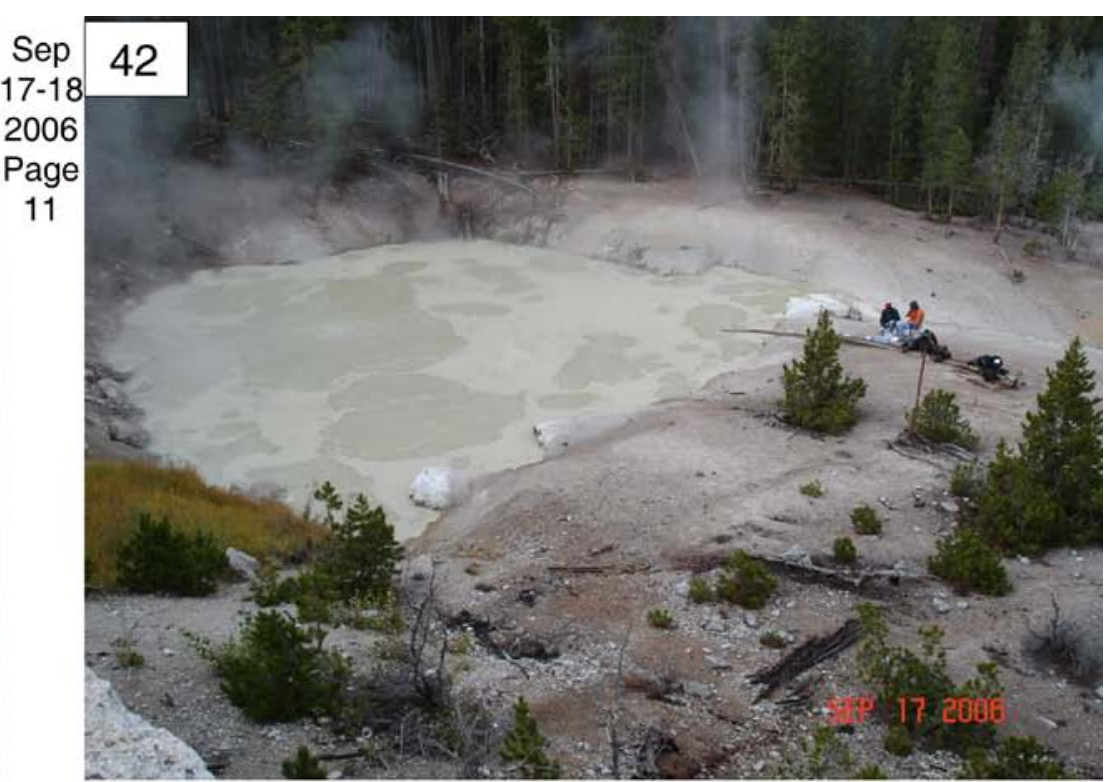

Joel Blum and Jim Ball at 06WA165, Turbulent Pool

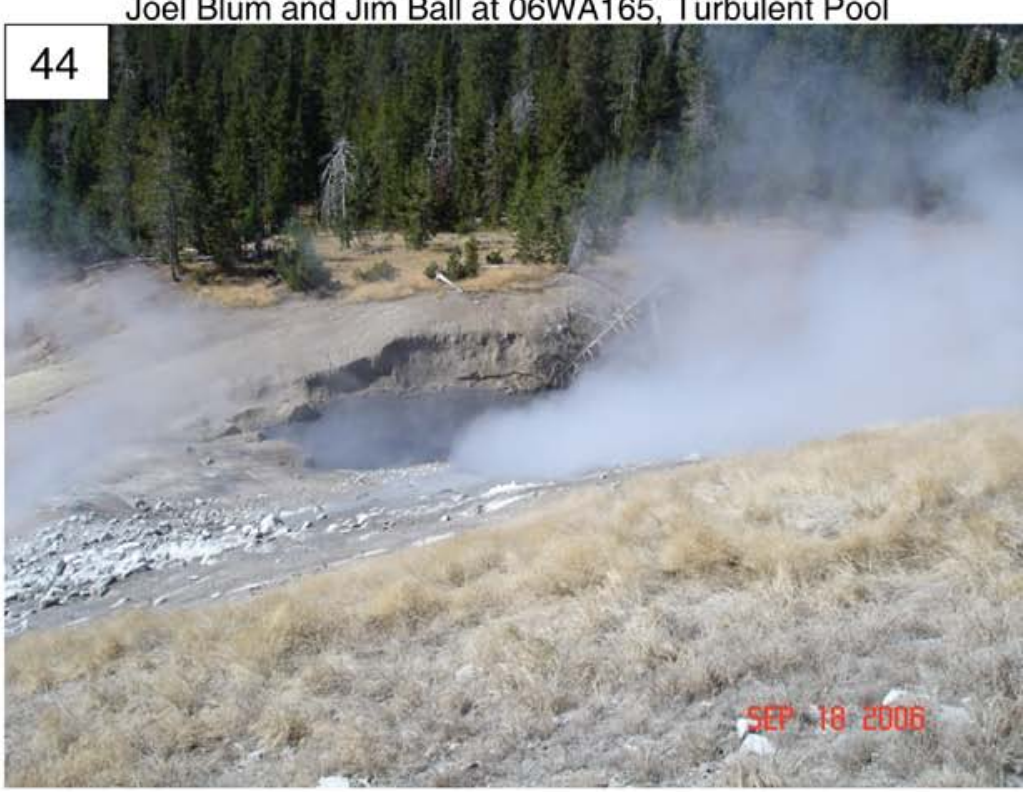

06WA168 


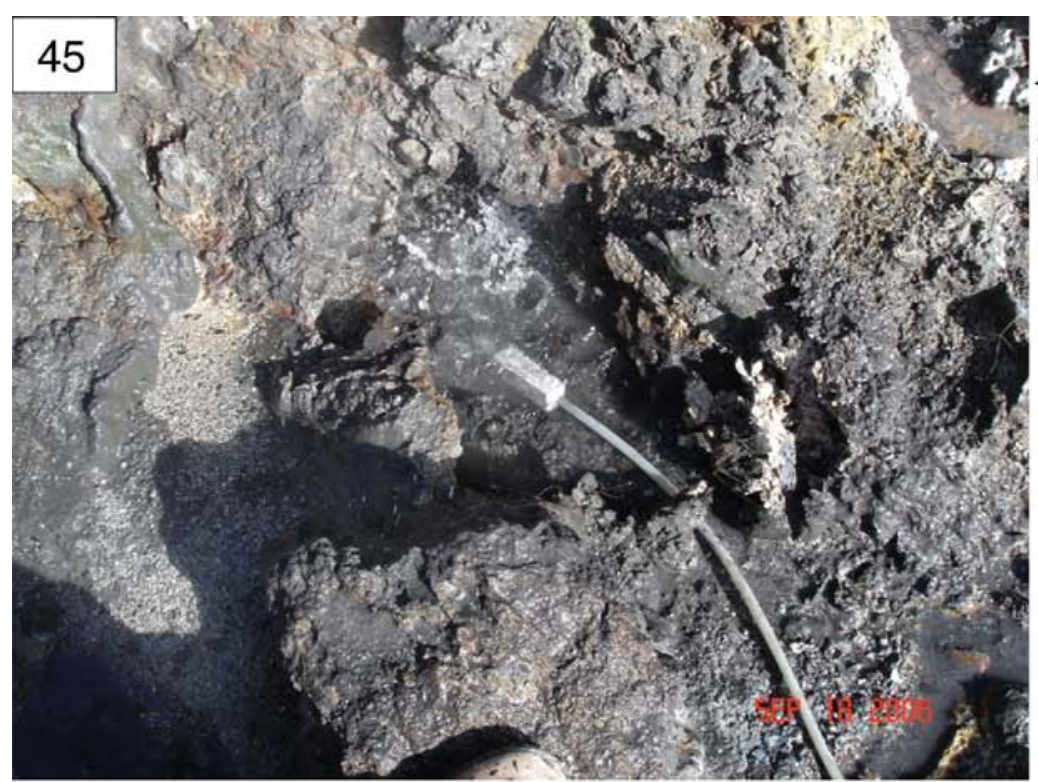

$\stackrel{9}{9}$

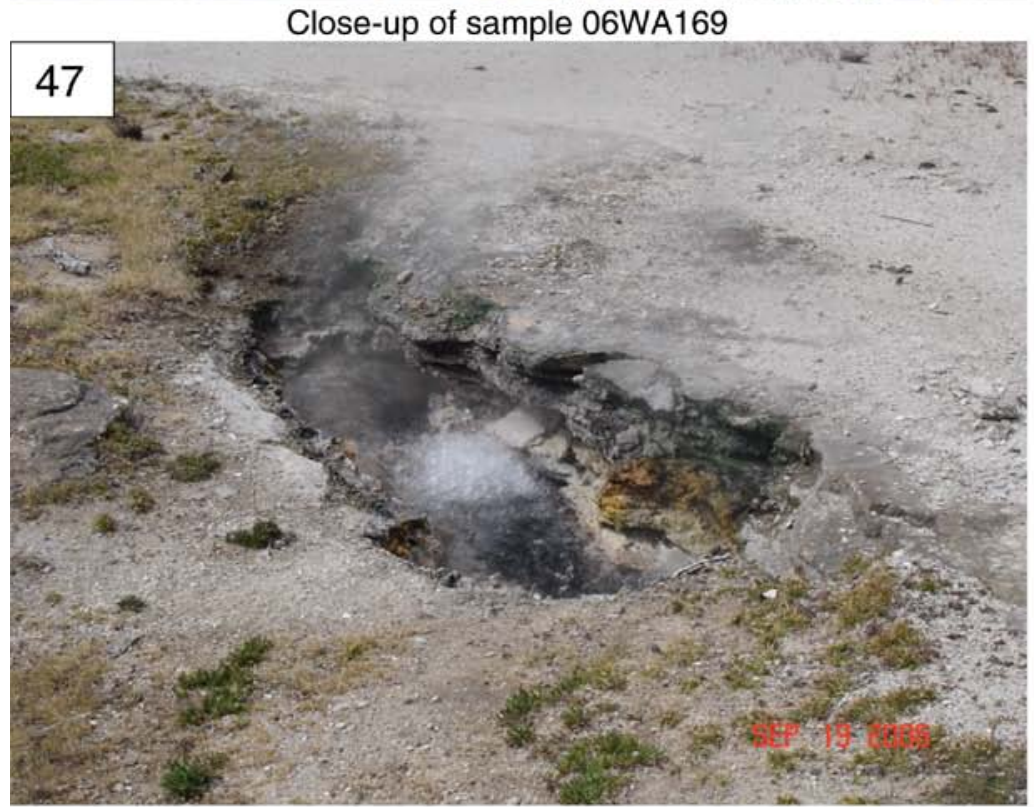

06WA171

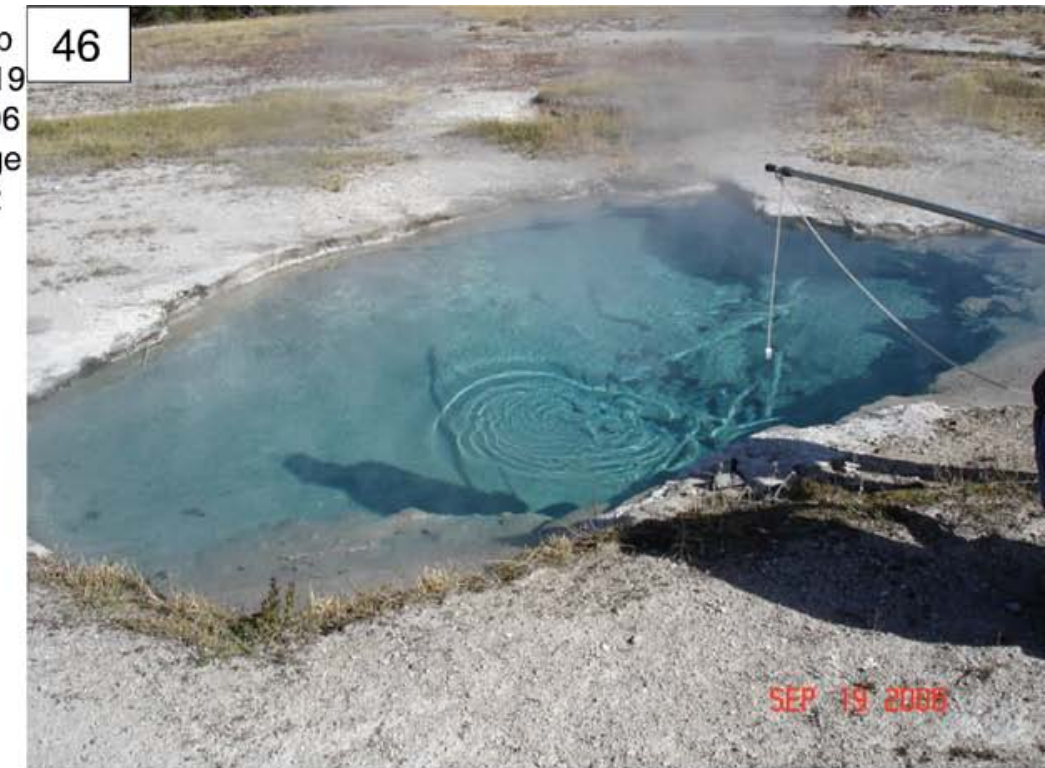

Sampling line in place at 06WA170

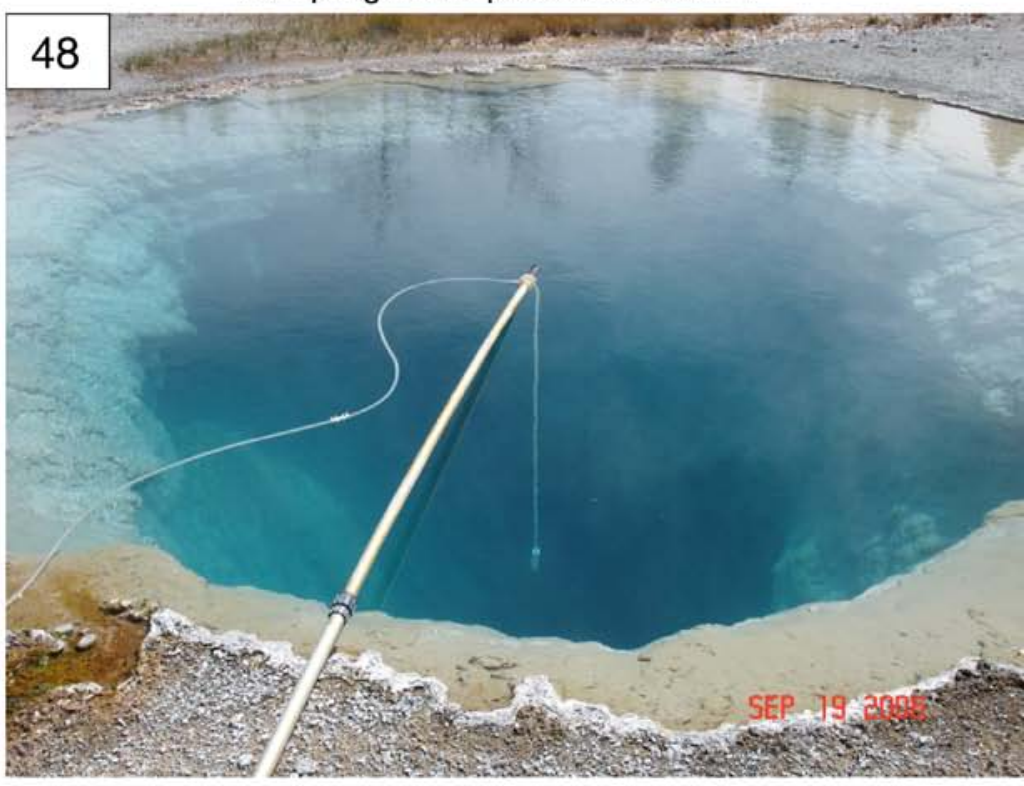

Sampling line in place at 06WA172 

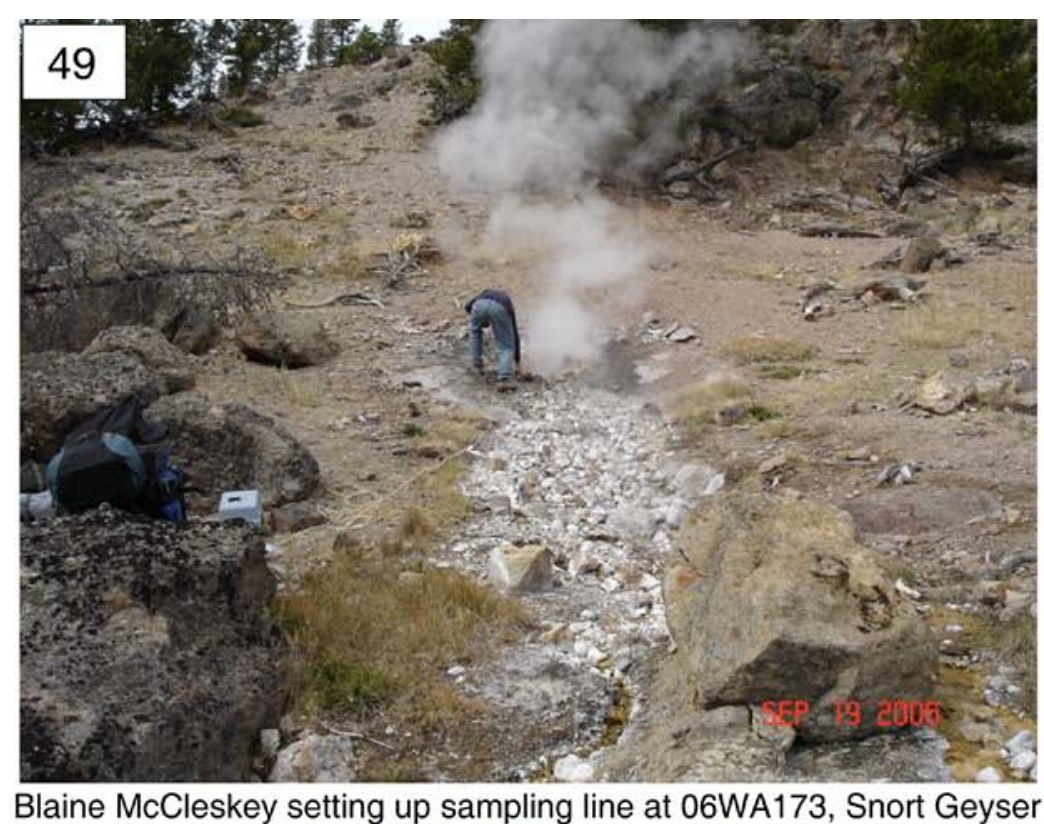

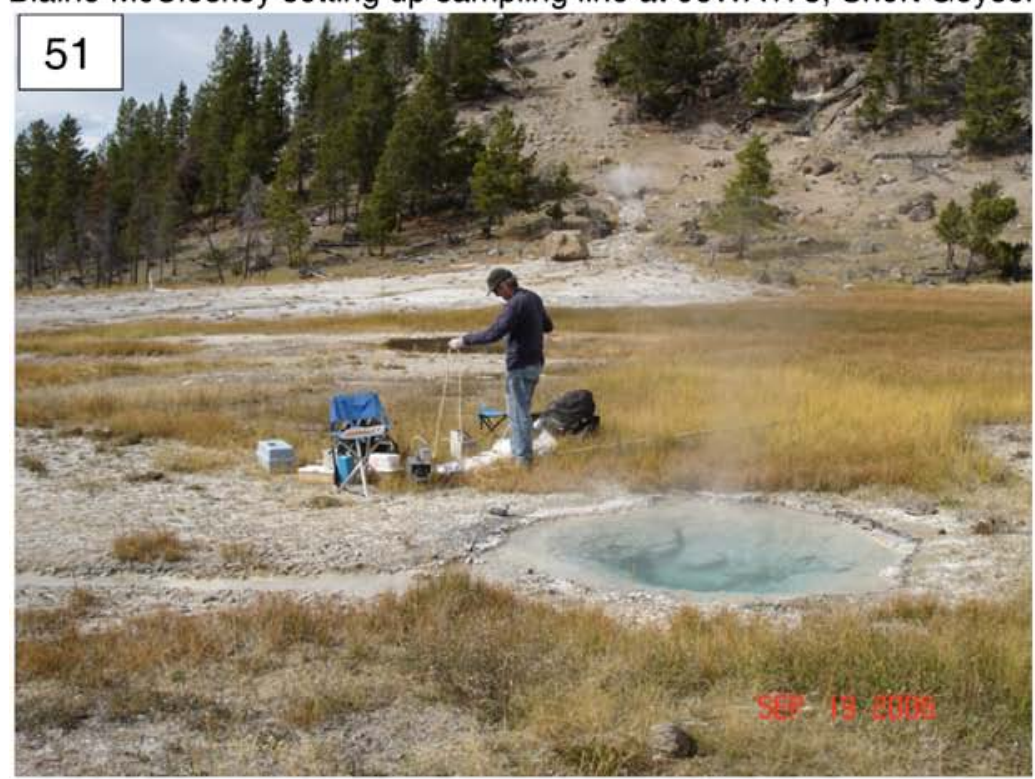

Blaine McCleskey setting up sampling line at 06WA174

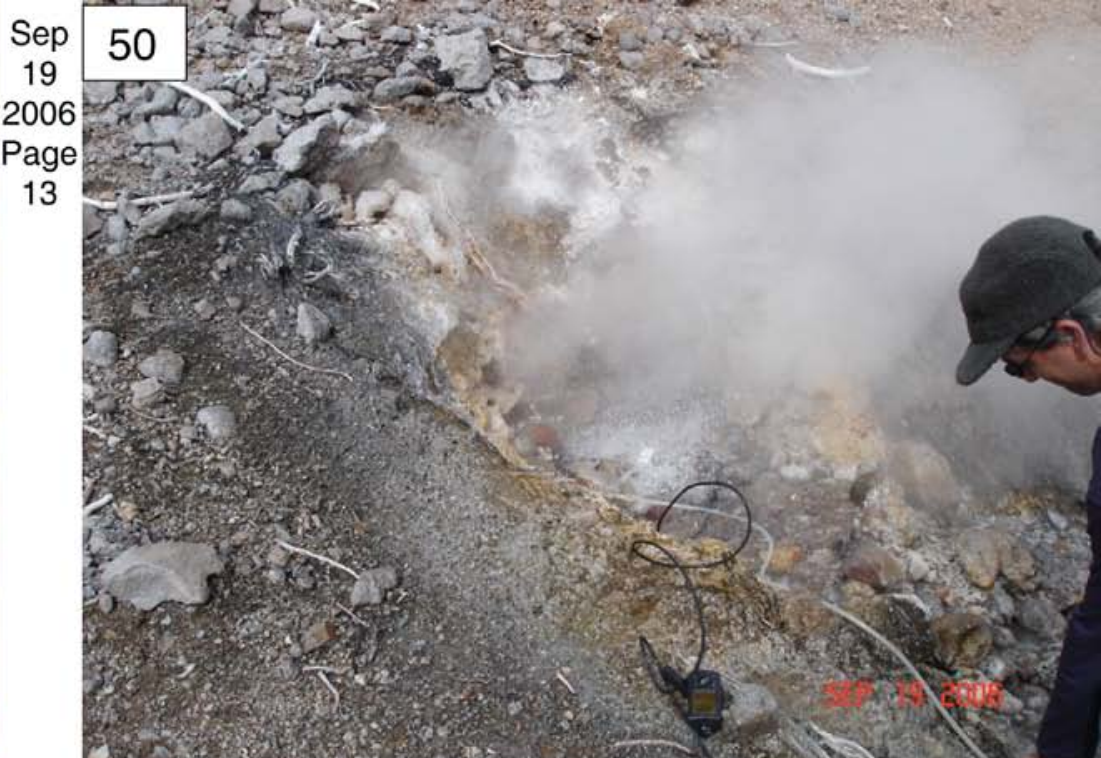

Blaine McCleskey setting up sampling line at 06WA173, Snort Geyser

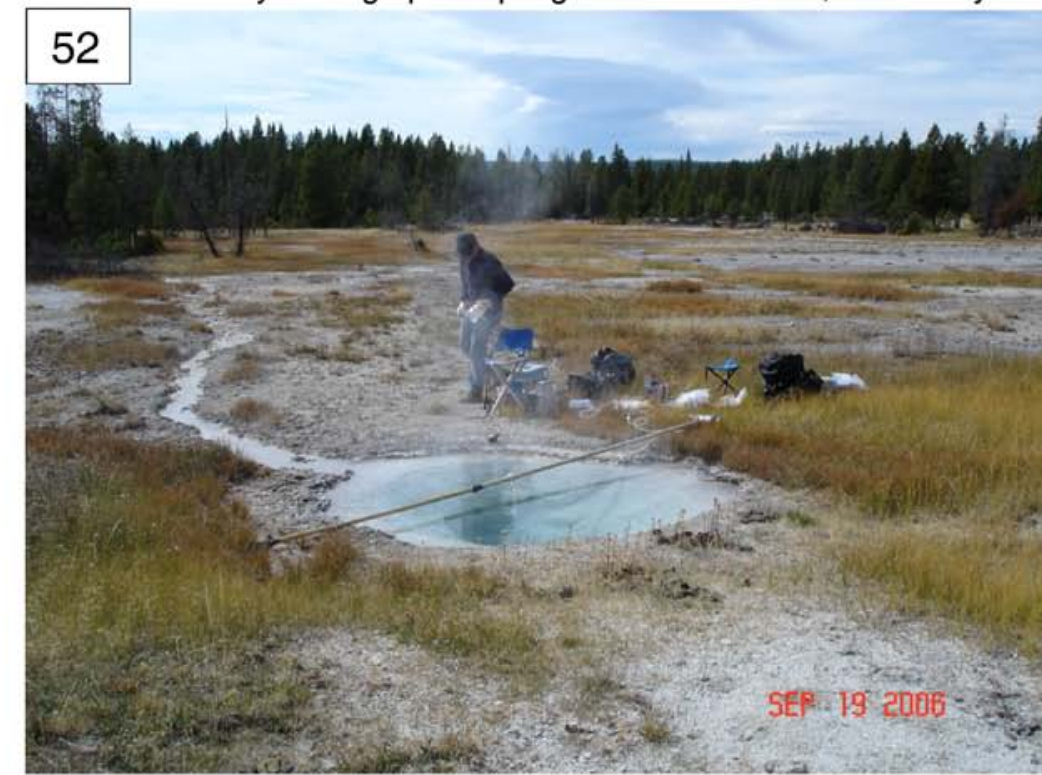

Blaine McCleskey sampling at 06WA174 

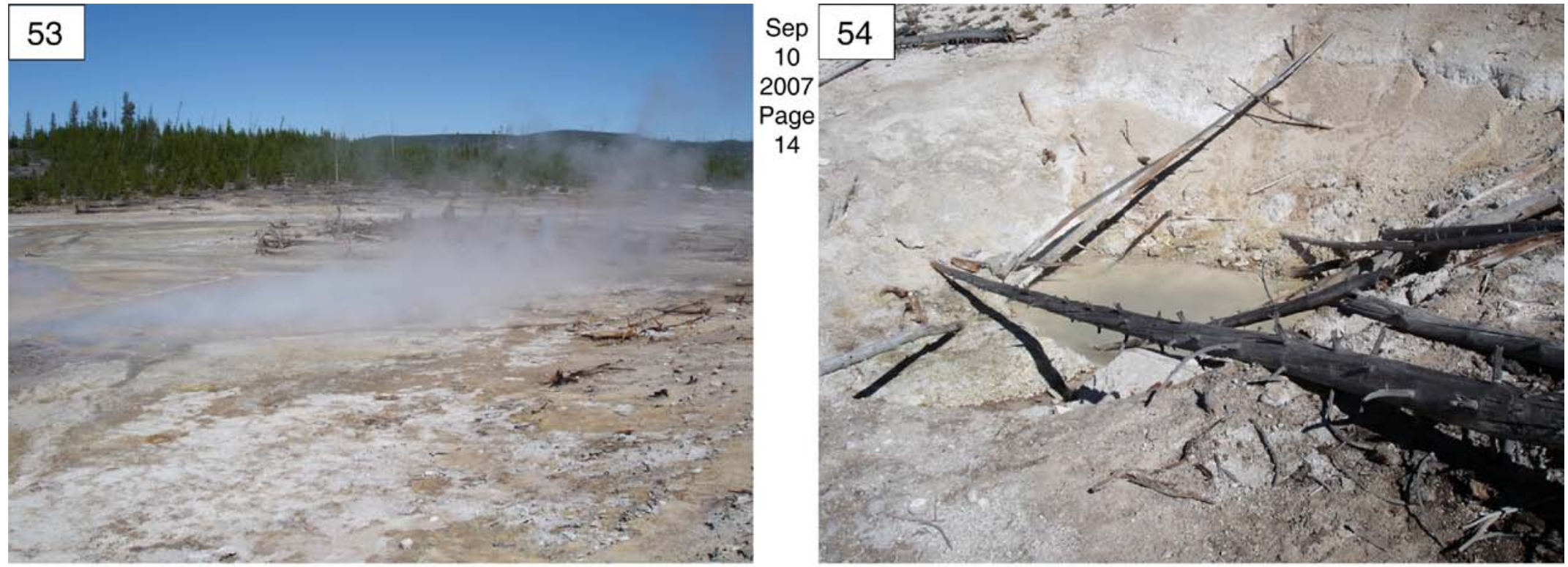

07WA105, "Persnickety Geyser"

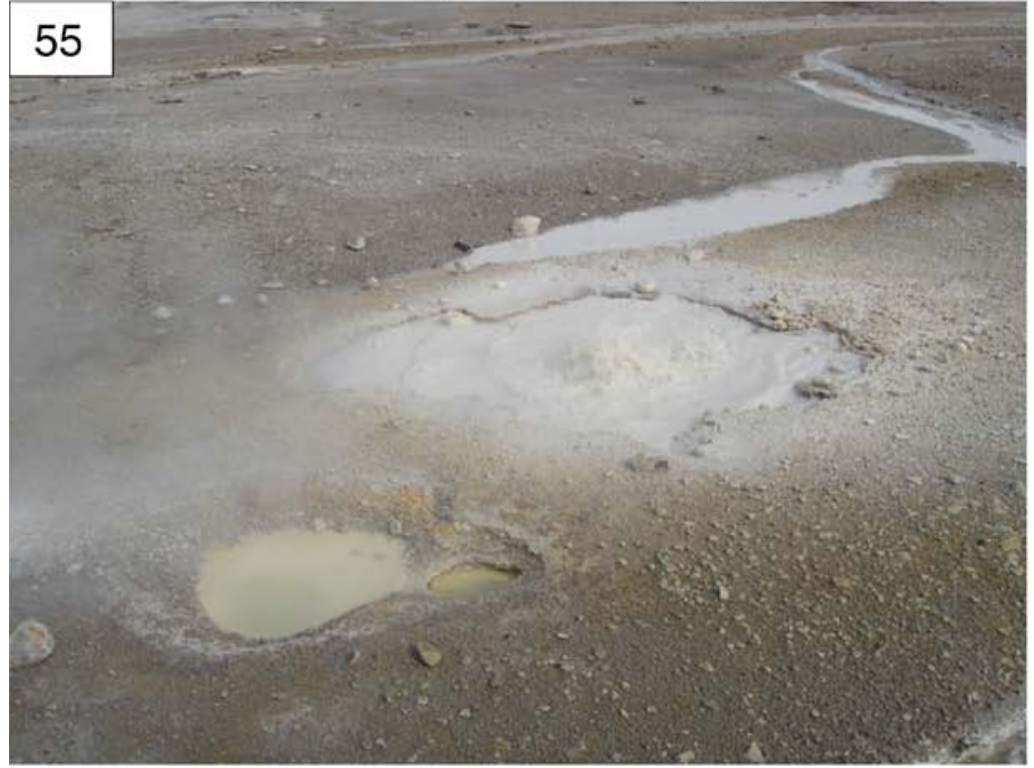

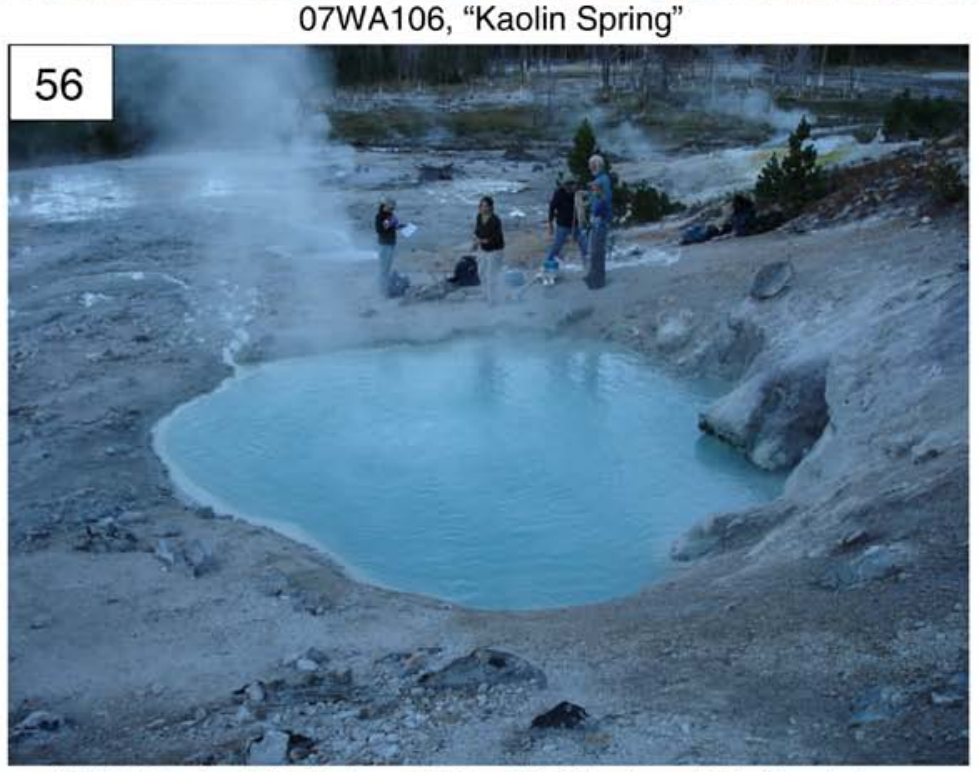

Nikki, Rachel Whittaker, Blaine McCleskey, Kirk Nordstrom, and Maria Pilar sampling at 07WA108 


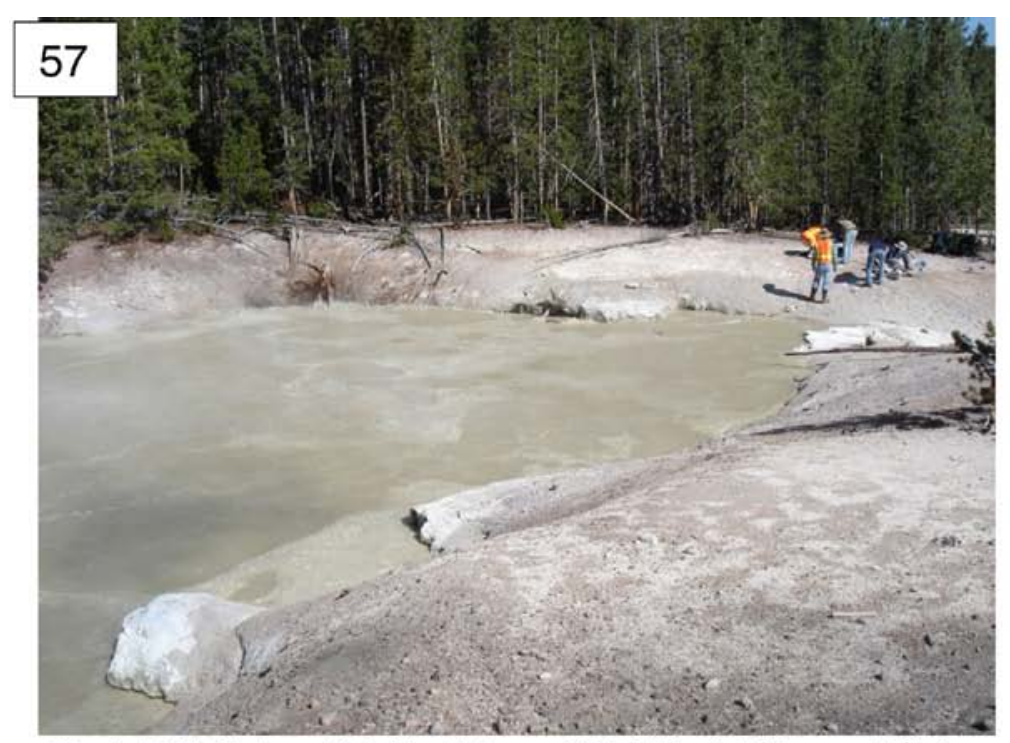

Rachel Whittaker, Kirk Nordstrom, Nikki, Maria Pilar, and Blain 용

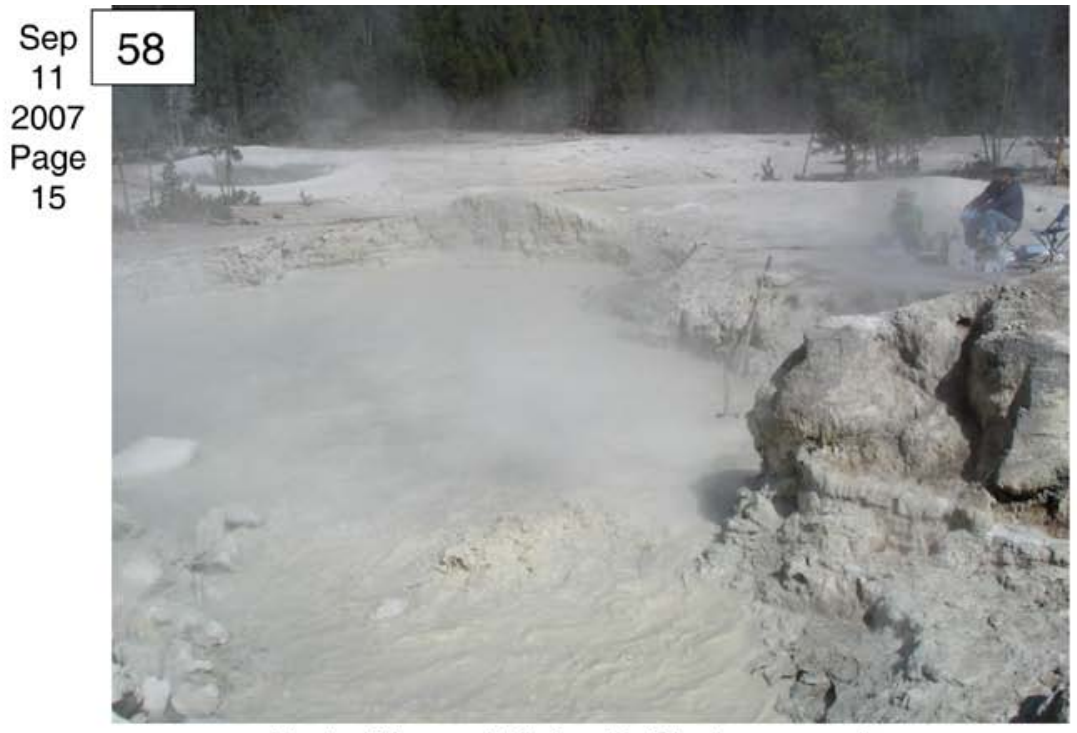

Sampling line in place at 07WA112
Maria Pilar and Blaine McCleskey sampling at 07WA110, Sulphur Caldron

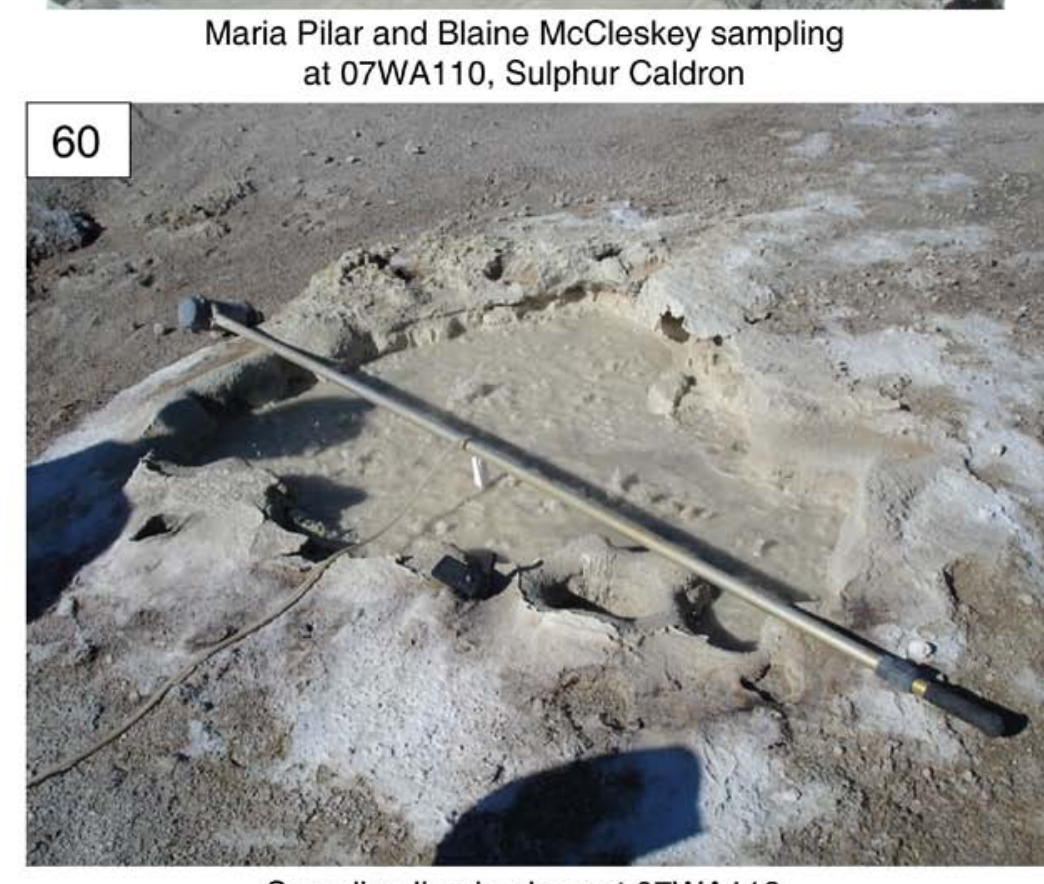

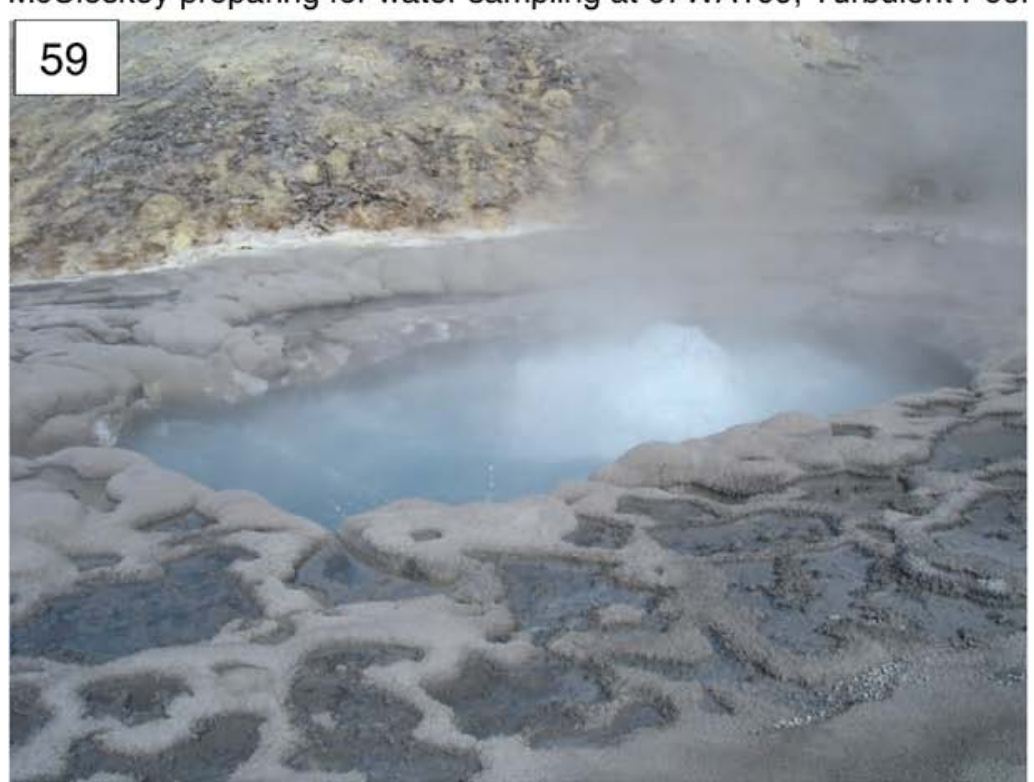

07WA111, Sulphur Spring 

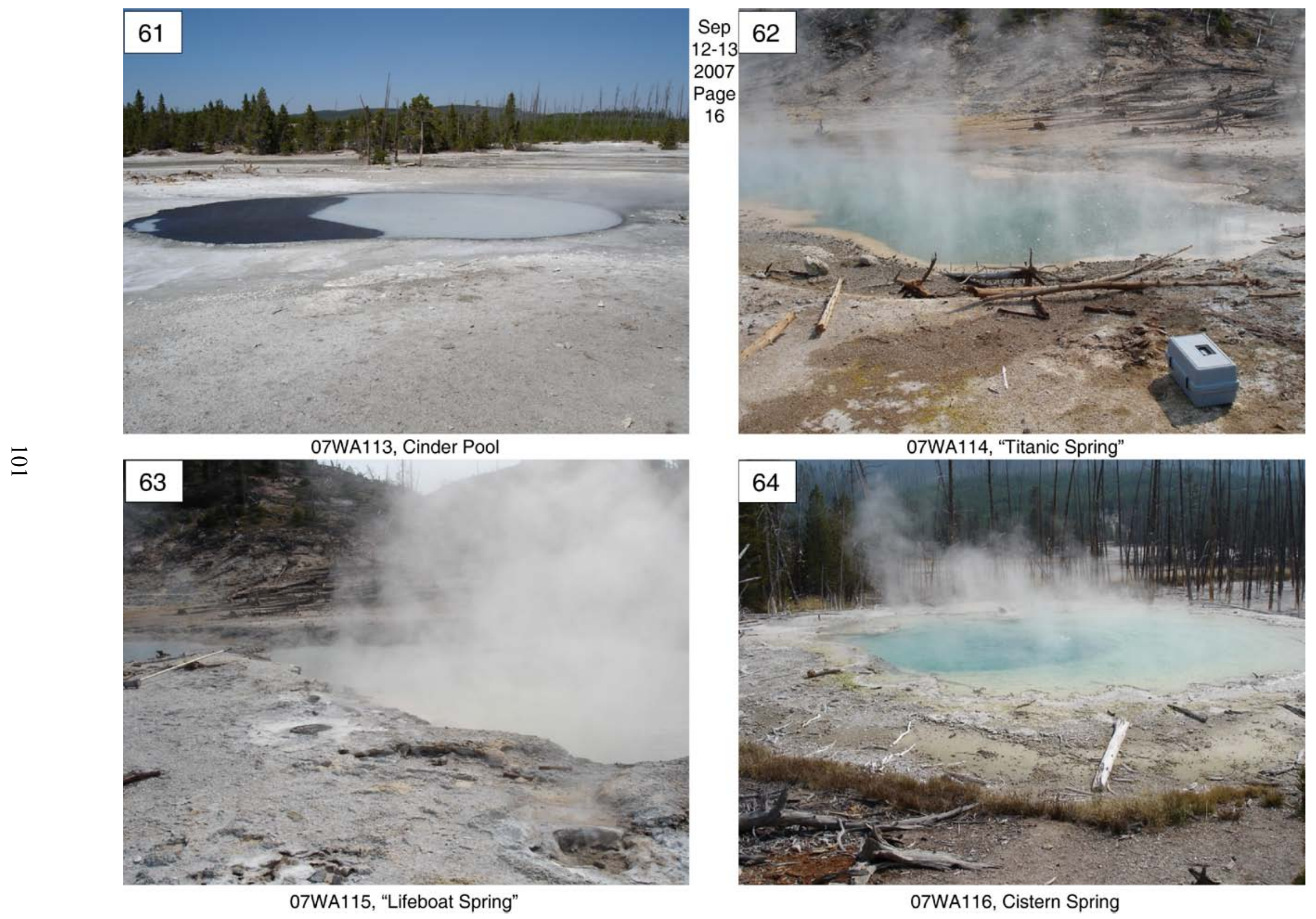

07WA116, Cistern Spring 


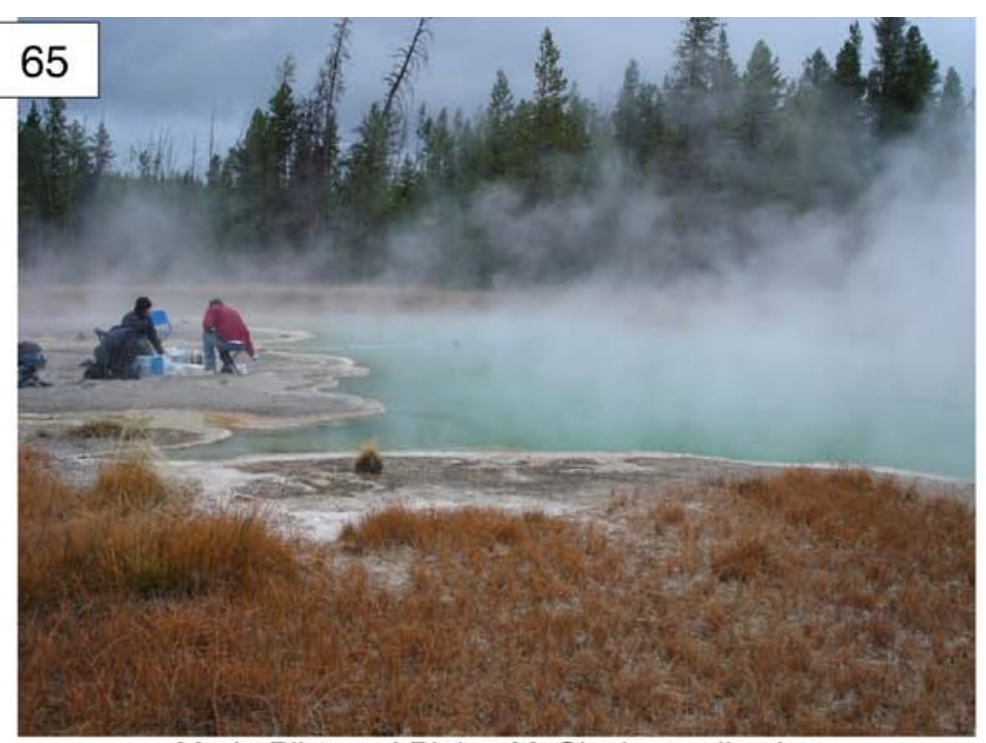

Maria Pilar and Blaine McCleskey collecting

오

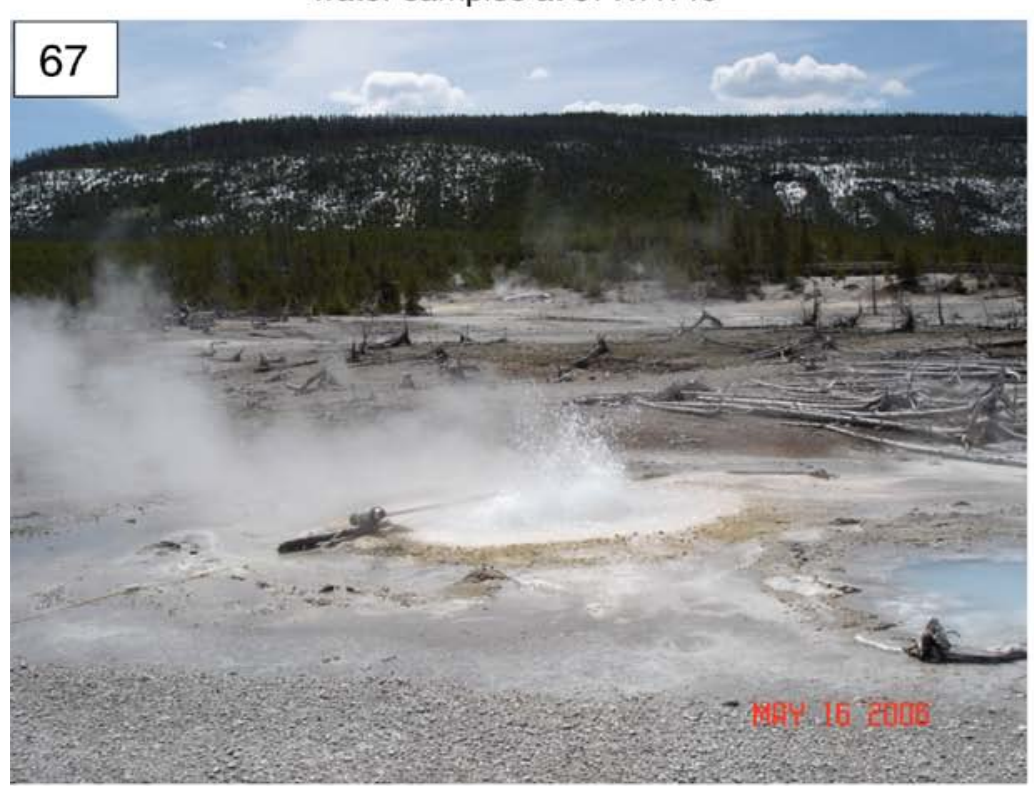

07WA142, "Second Eruptor"

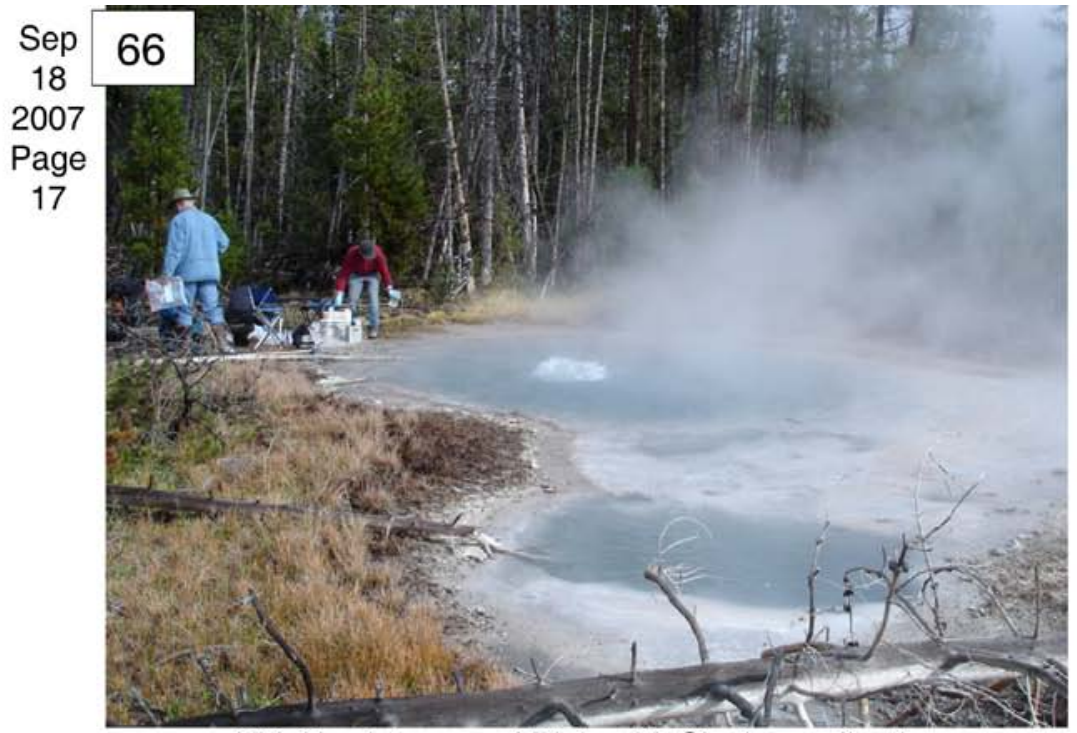

Kirk Nordstrom and Blaine McCleskey collecting water samples at 07WA141

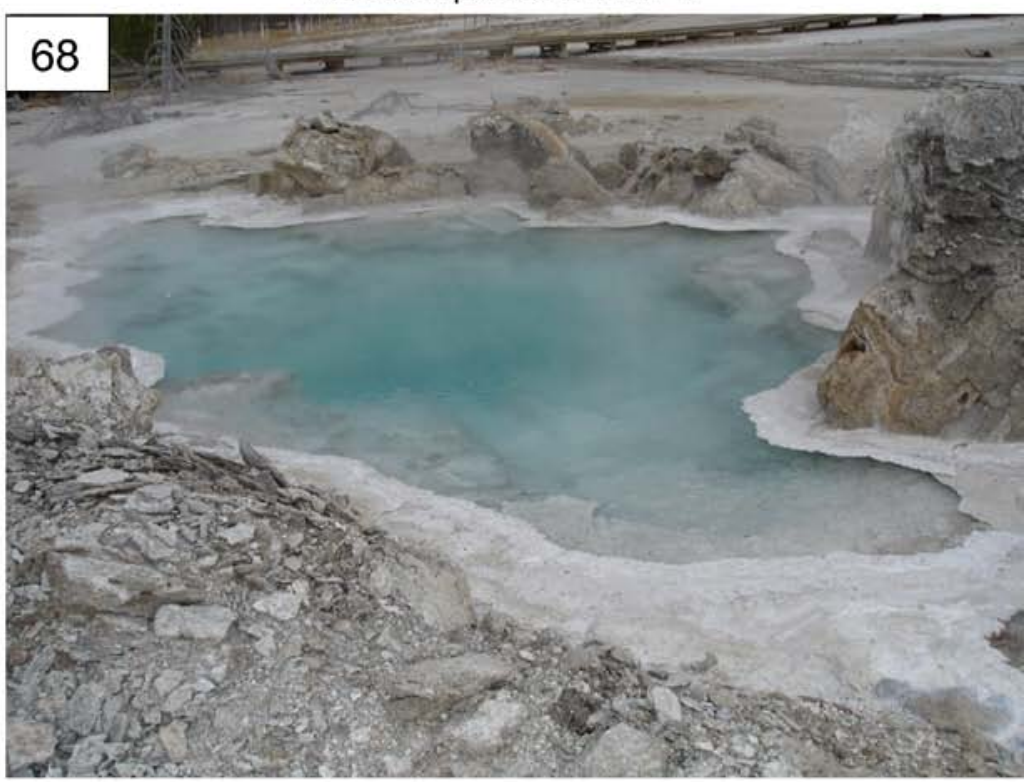

07WA143, Porkchop Geyser 


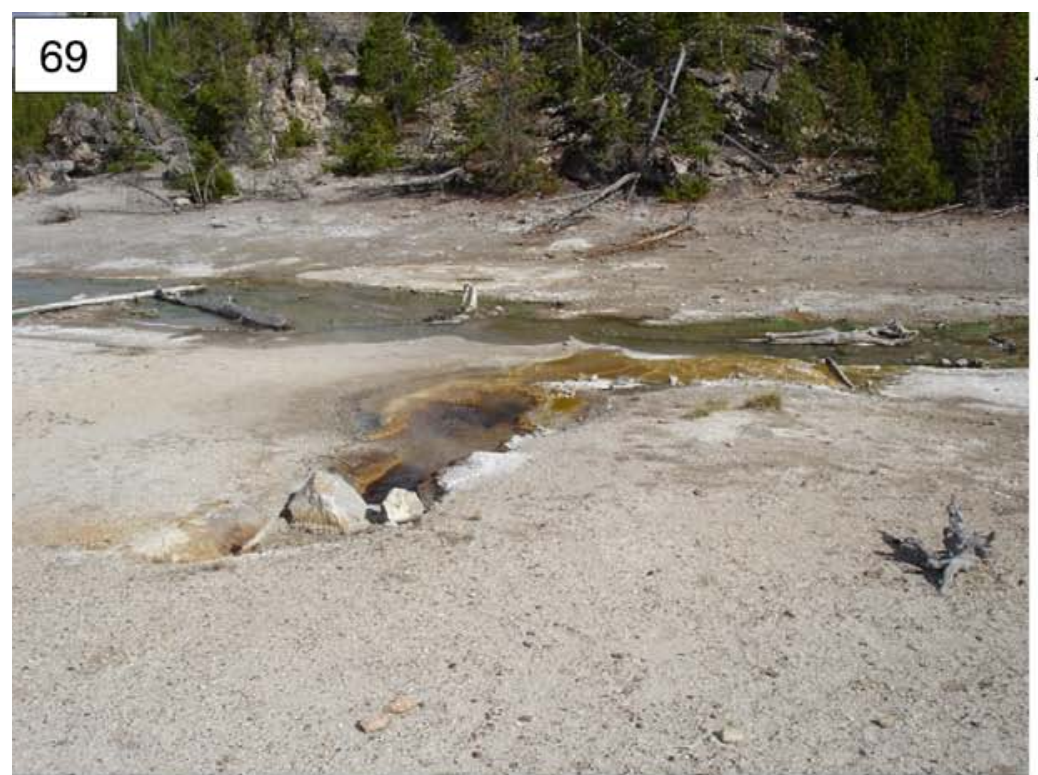

$\overline{\mathrm{s}}$

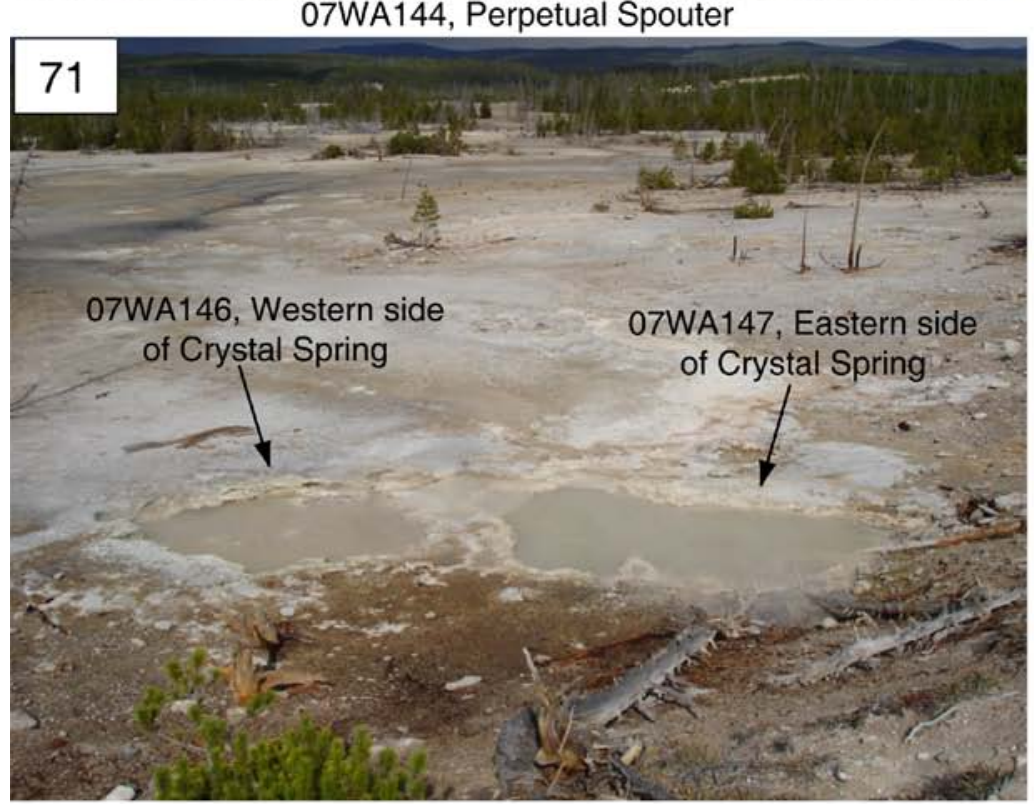

07WA146 and 07WA147, Crystal Spring

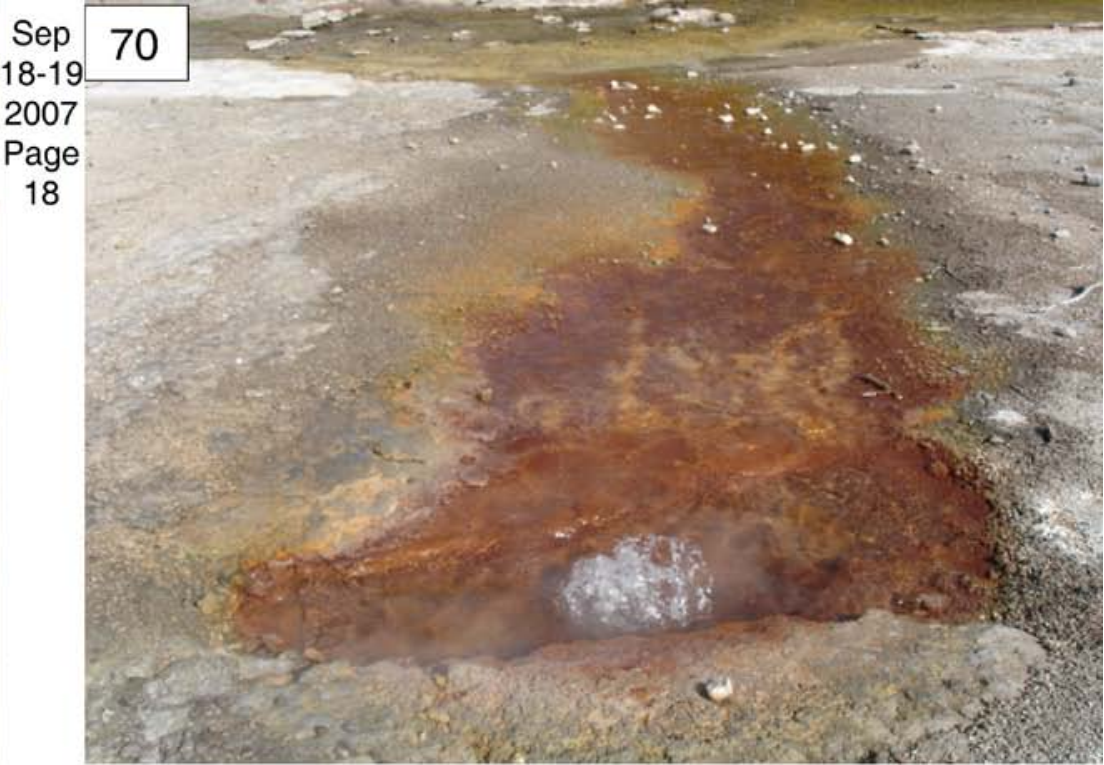

07WA145

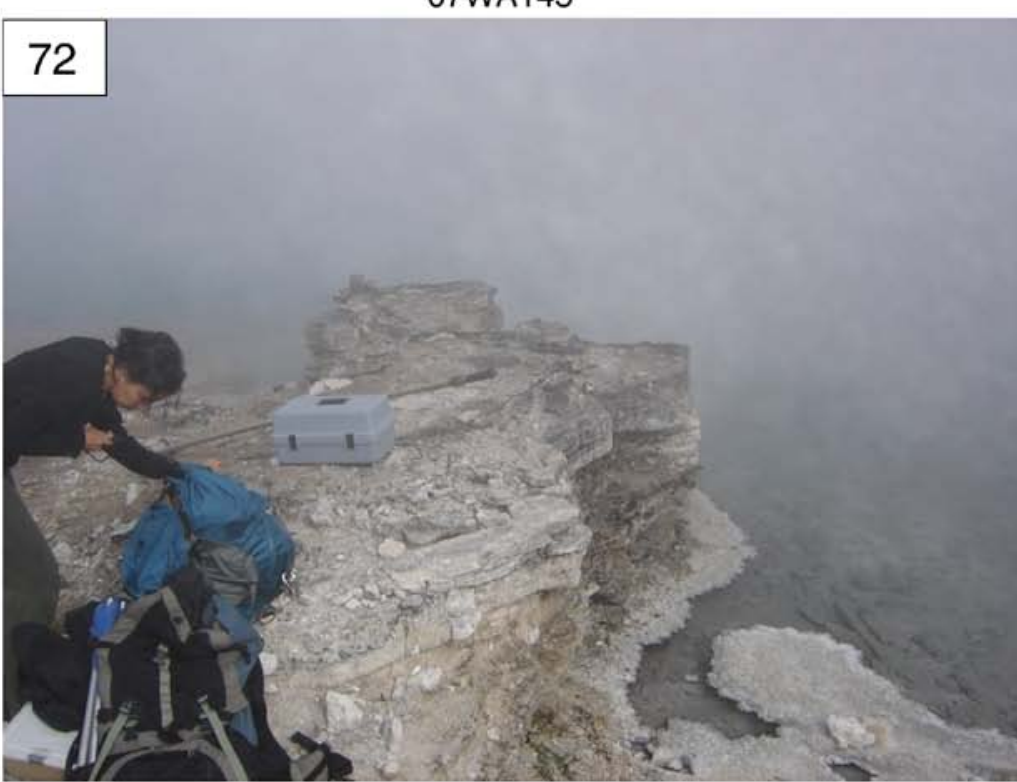

Maria Pilar sampling at 07WA148, Excelsior Geyser Crater 


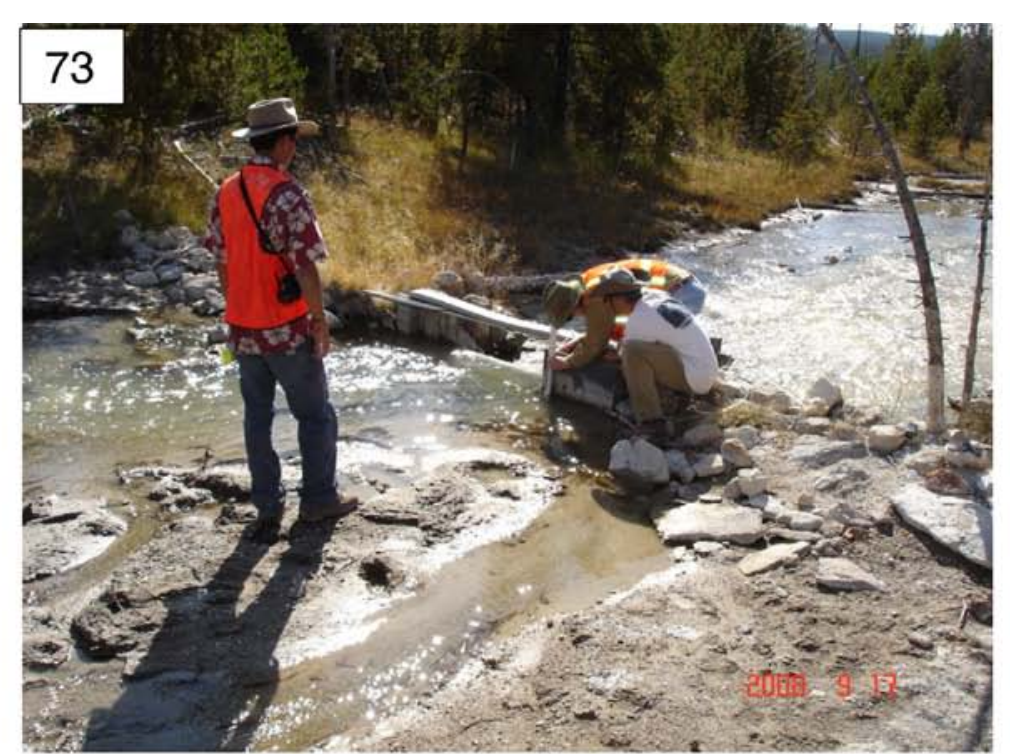

$\bar{\ddagger}$

Randall Chiu, Kirk Nordstrom, and Blaine McCleskey positioning an automated data logger at 08WA105, Tantalus Creek at weir

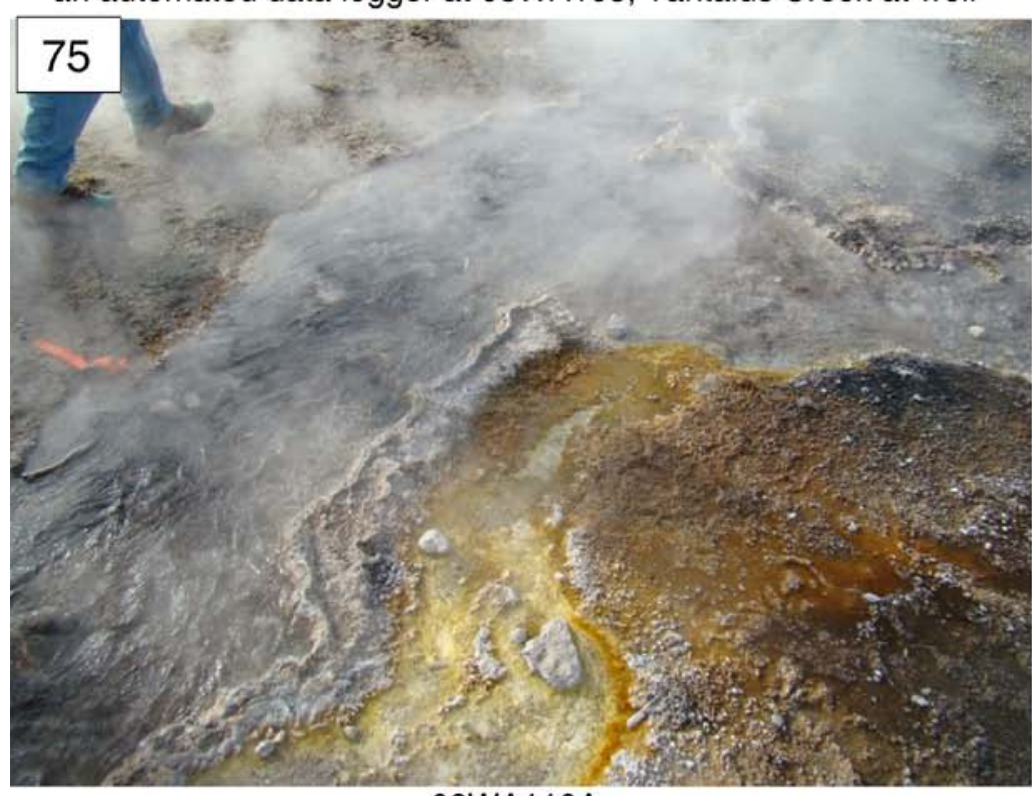

08WA110A

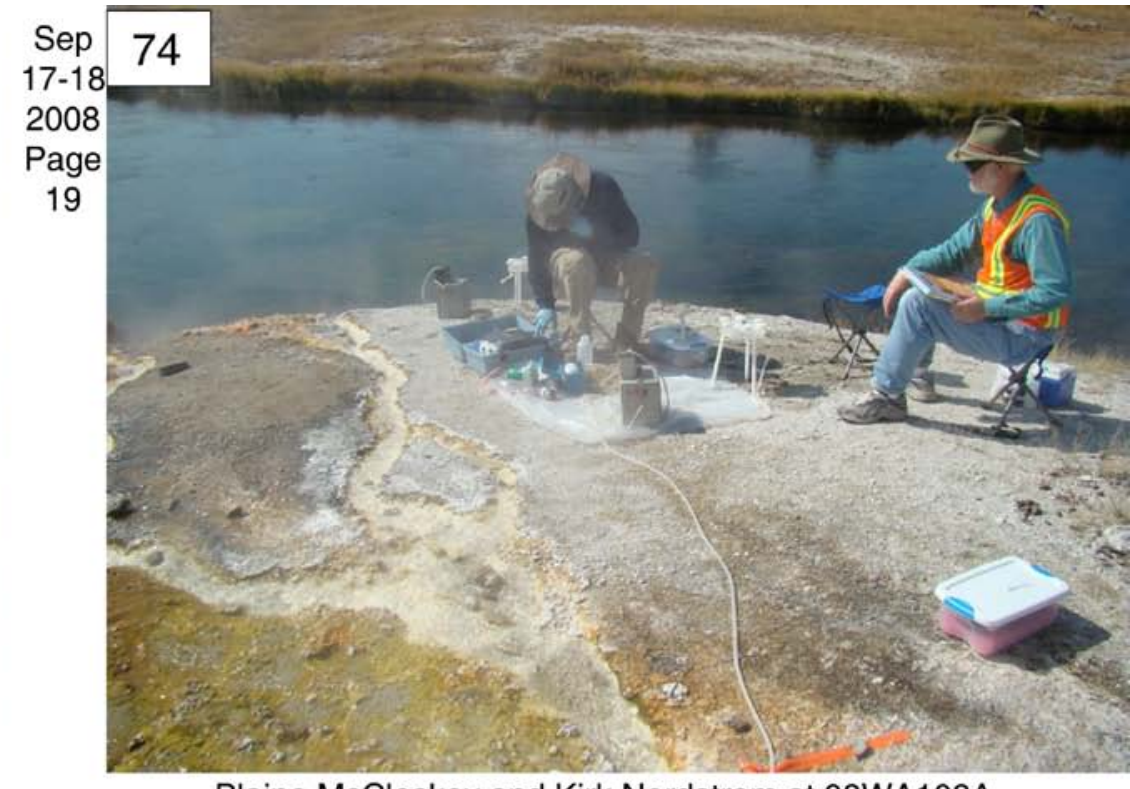

Blaine McCleskey and Kirk Nordstrom at 08WA108A

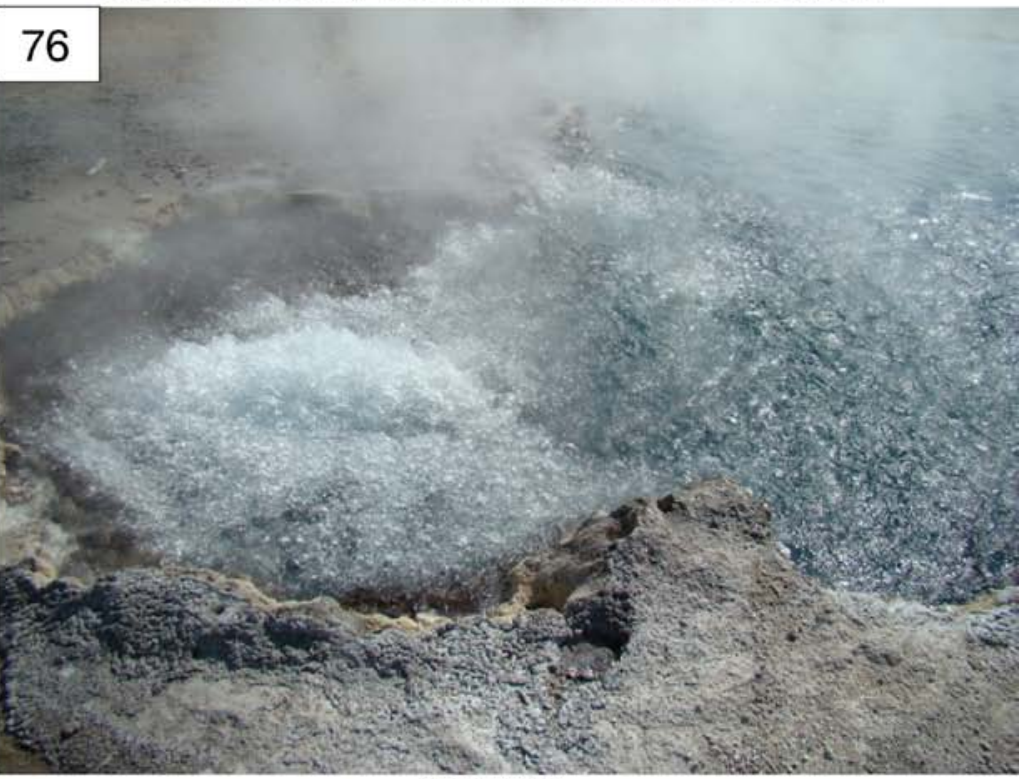

08WA112 


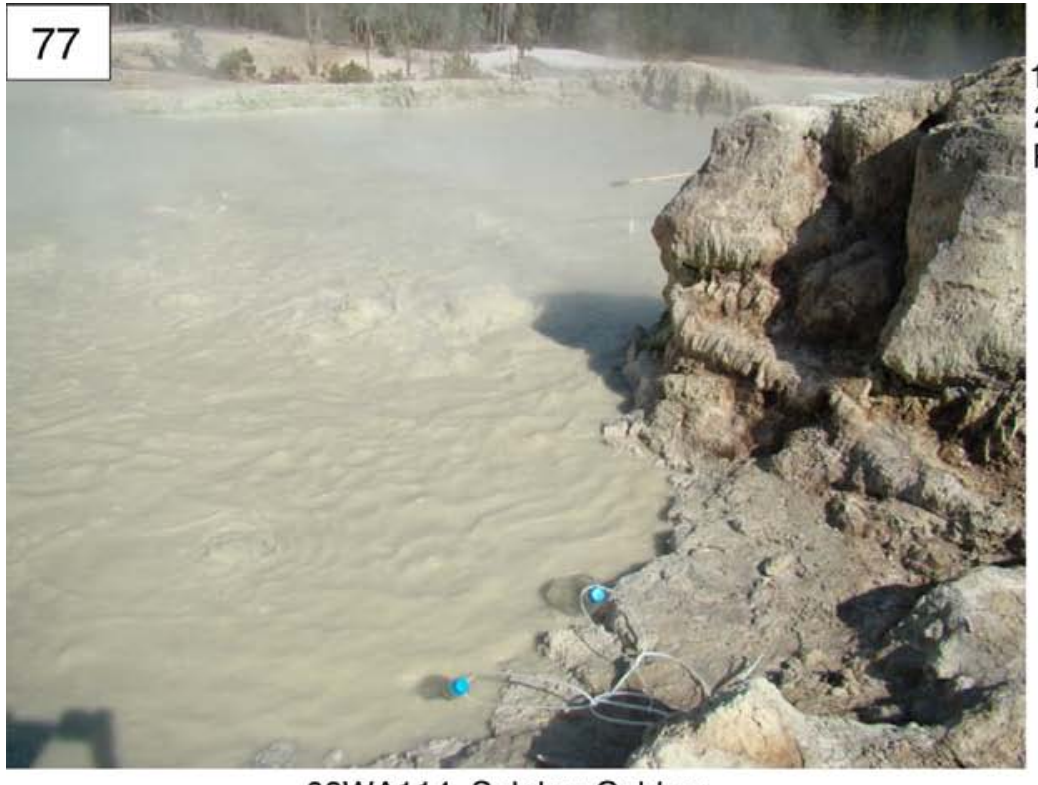

$\ddot{\circ}$

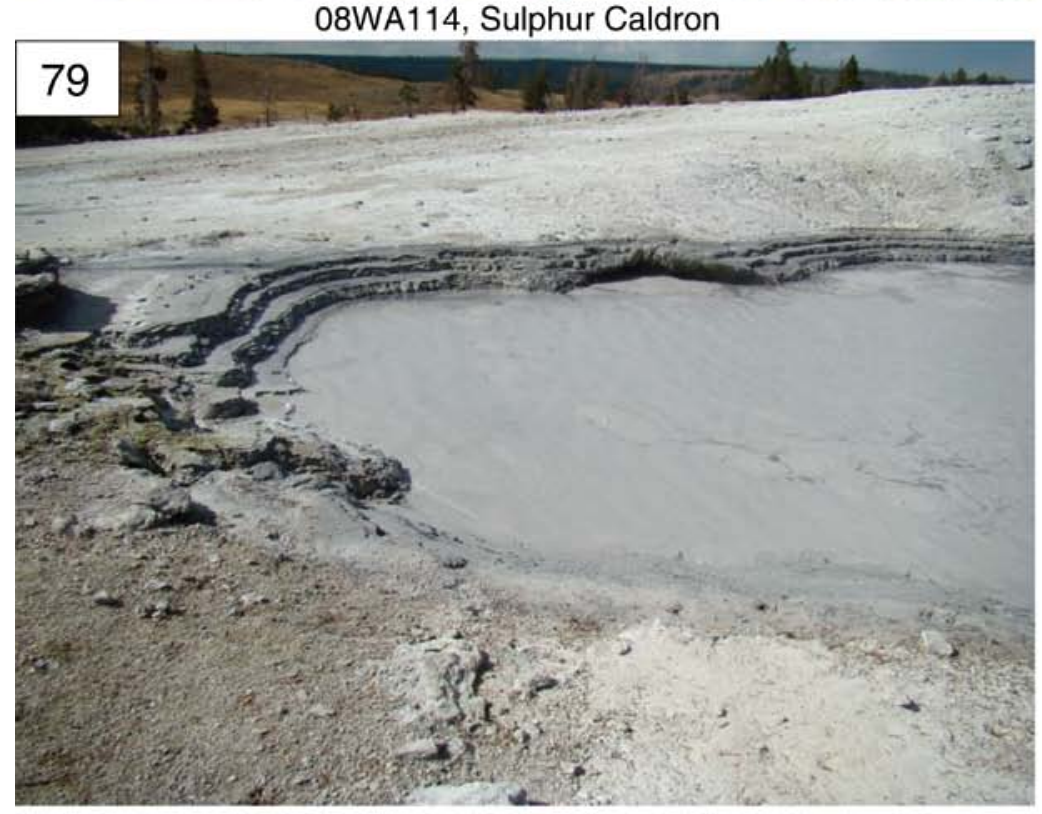

08WA116
Sep 78

$19-20$

2008

Page

20

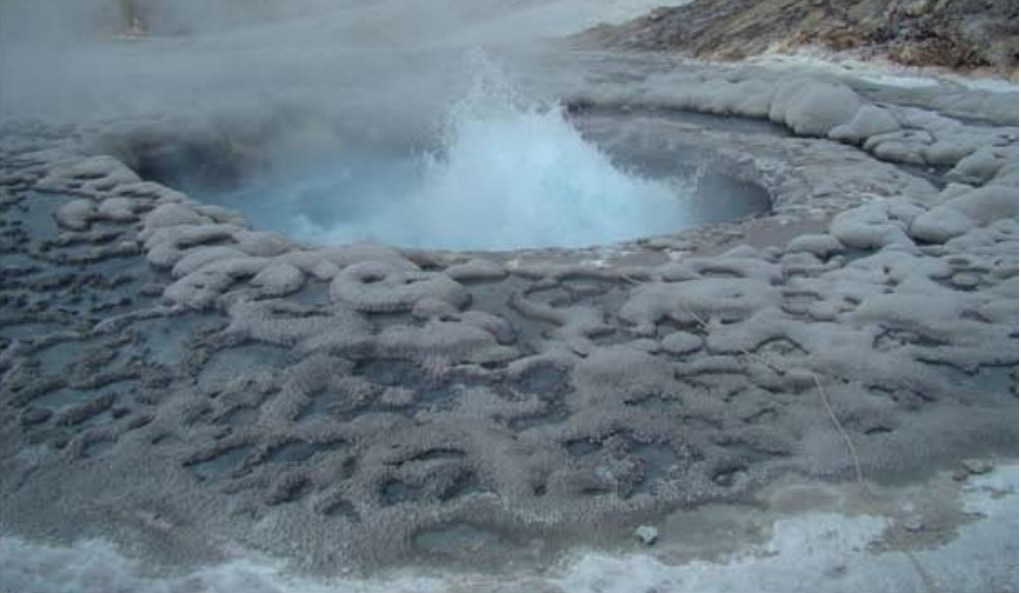

08WA115, Sulphur Spring

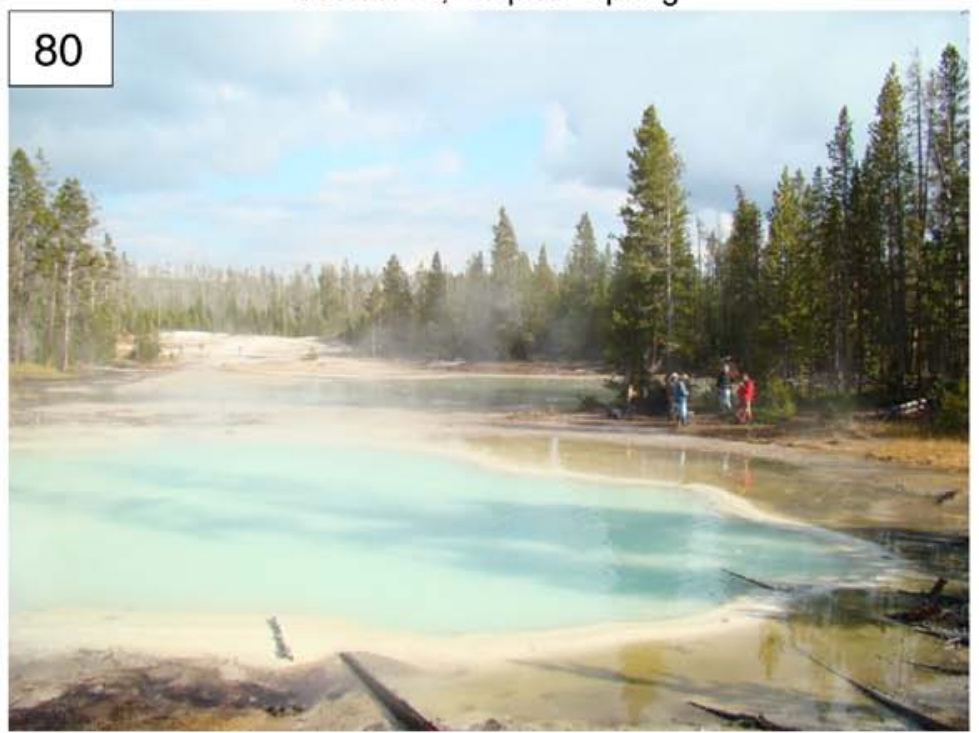

Nuno Durães, Kirk Nordstrom, Jim Ball, and Blaine McCleskey preparing to collect water samples at 08WA118 


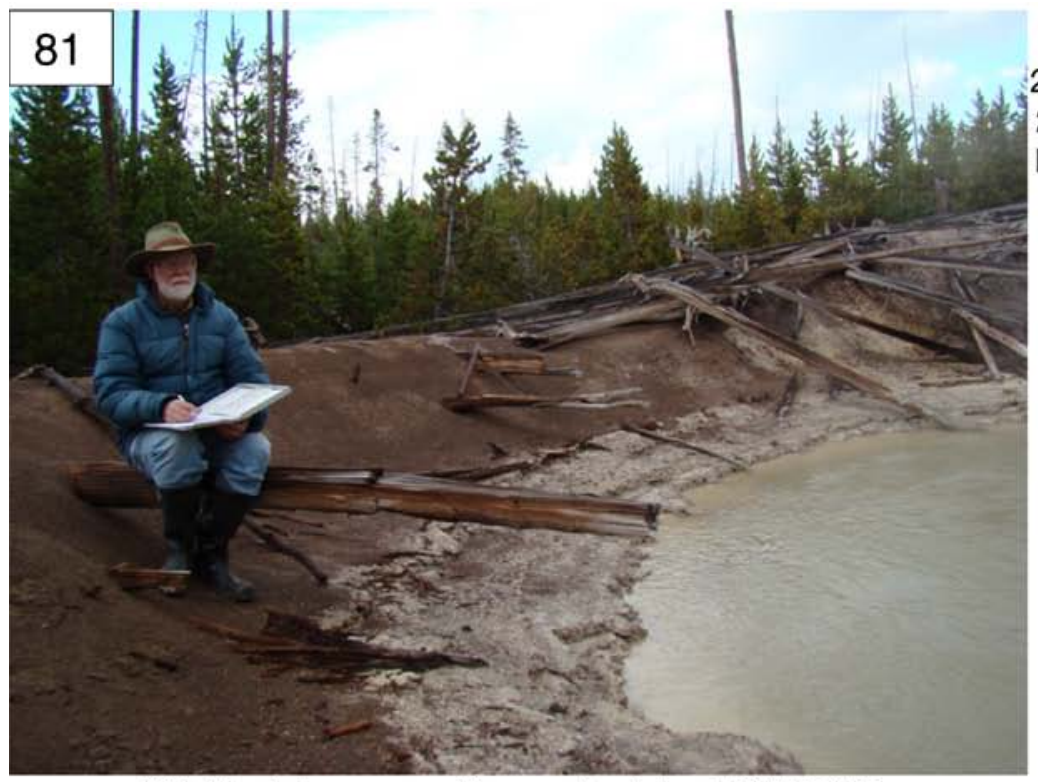

ळ

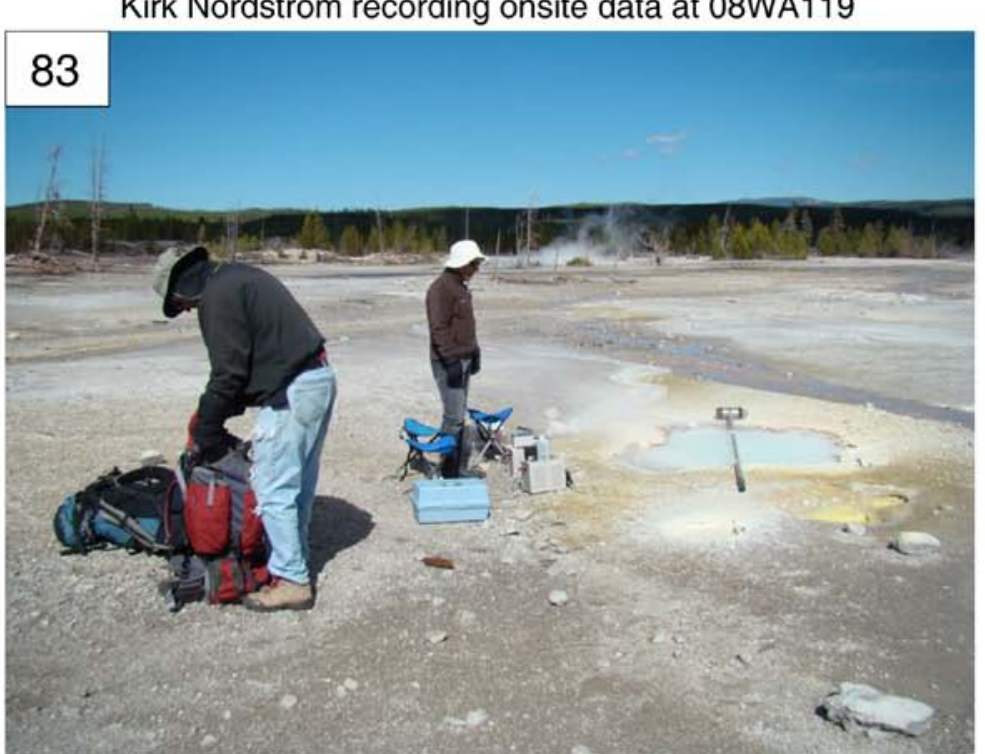

Jim Ball and Nuno Durães preparing to collect water samples at 08WA121

\section{2}

20-23

2008

Page

21

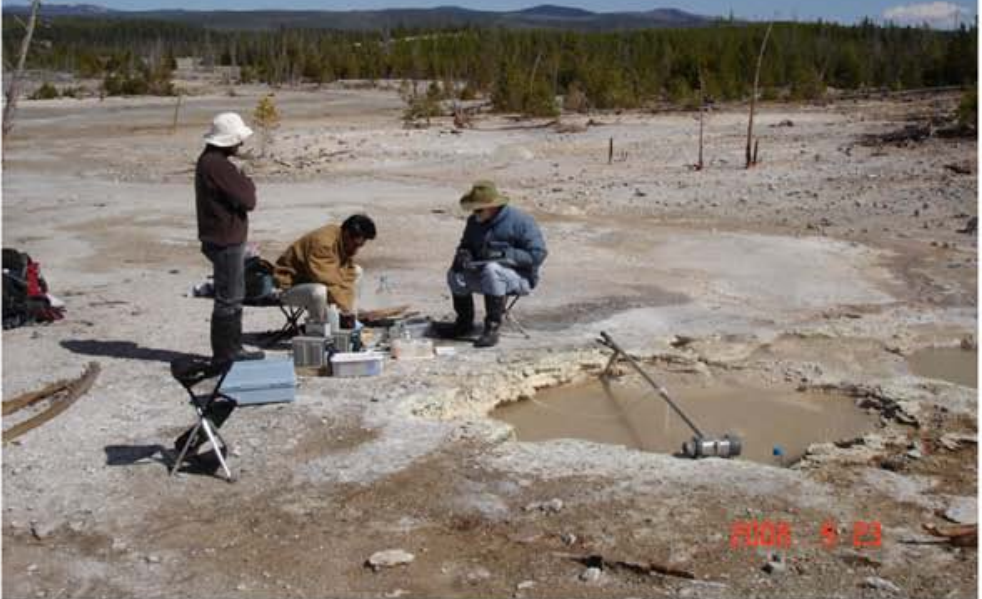

Nuno Durães, Randall Chiu, and Kirk Nordstrom collecting water samples at 08 WA120

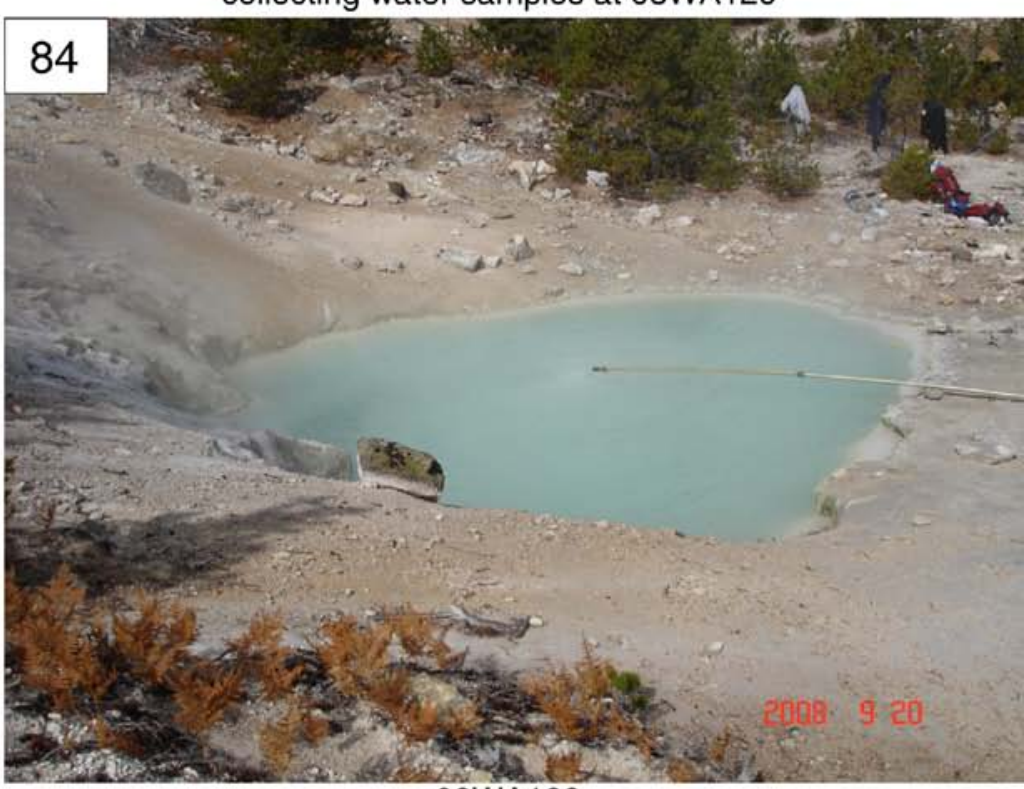

08WA122 


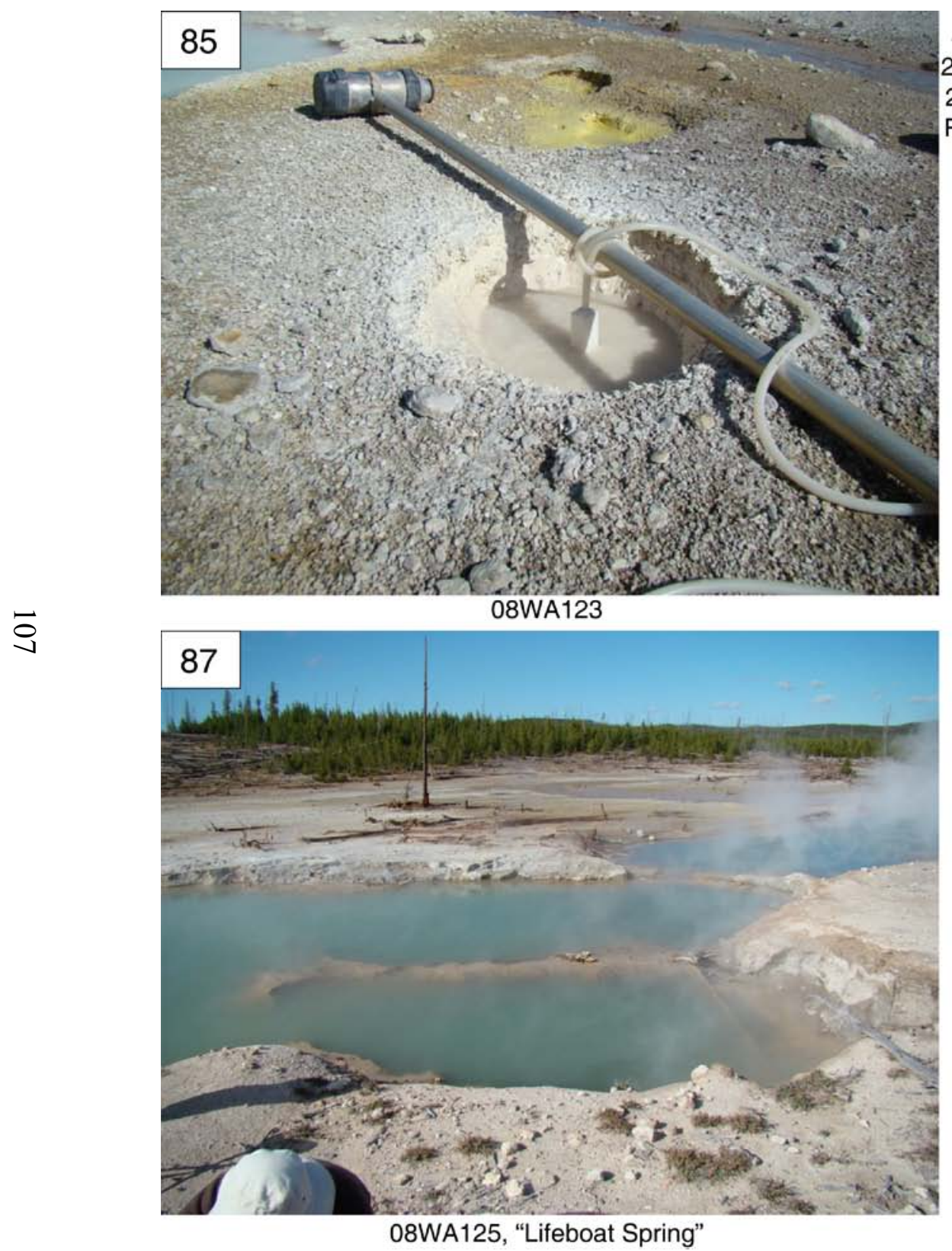

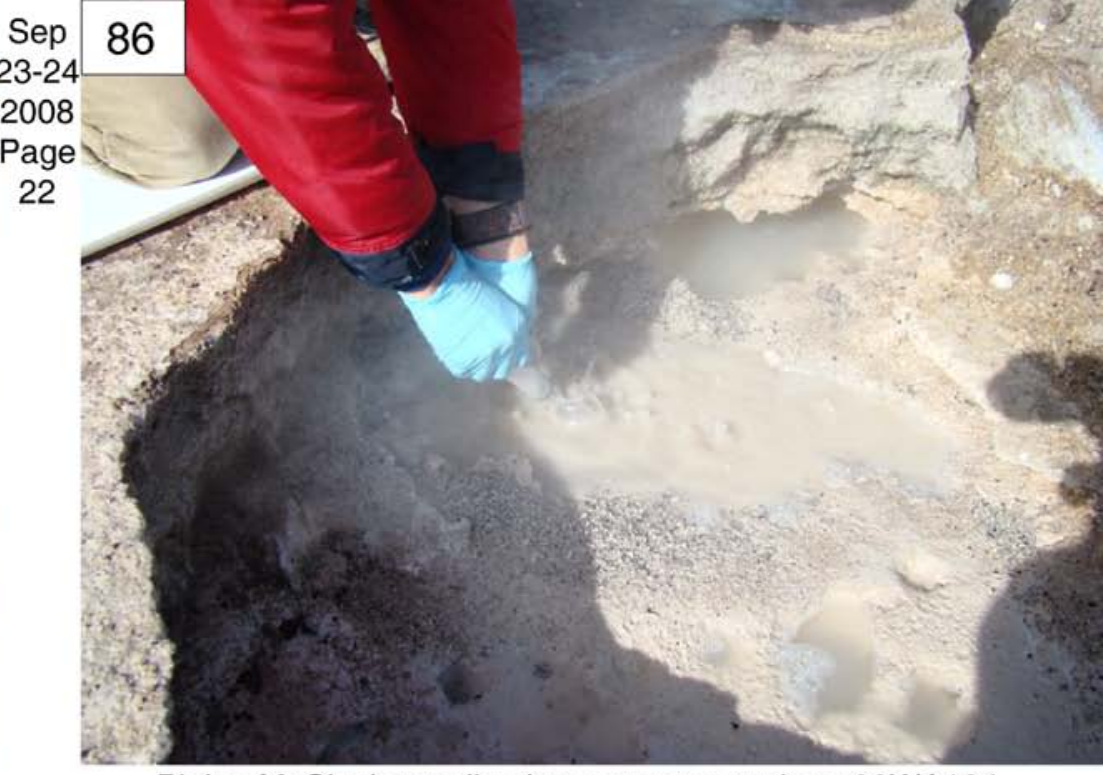

Blaine McCleskey collecting a water sample at 08WA124

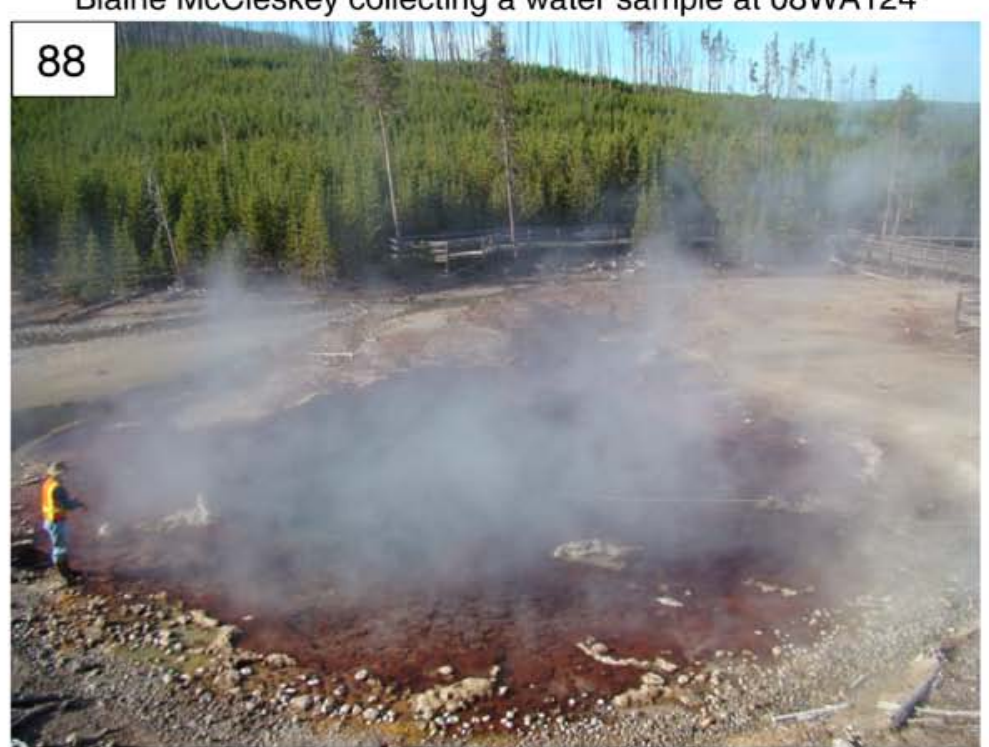

Kirk Nordstrom positioning the sample intake tubing at 08WA126, Echinus Geyser 


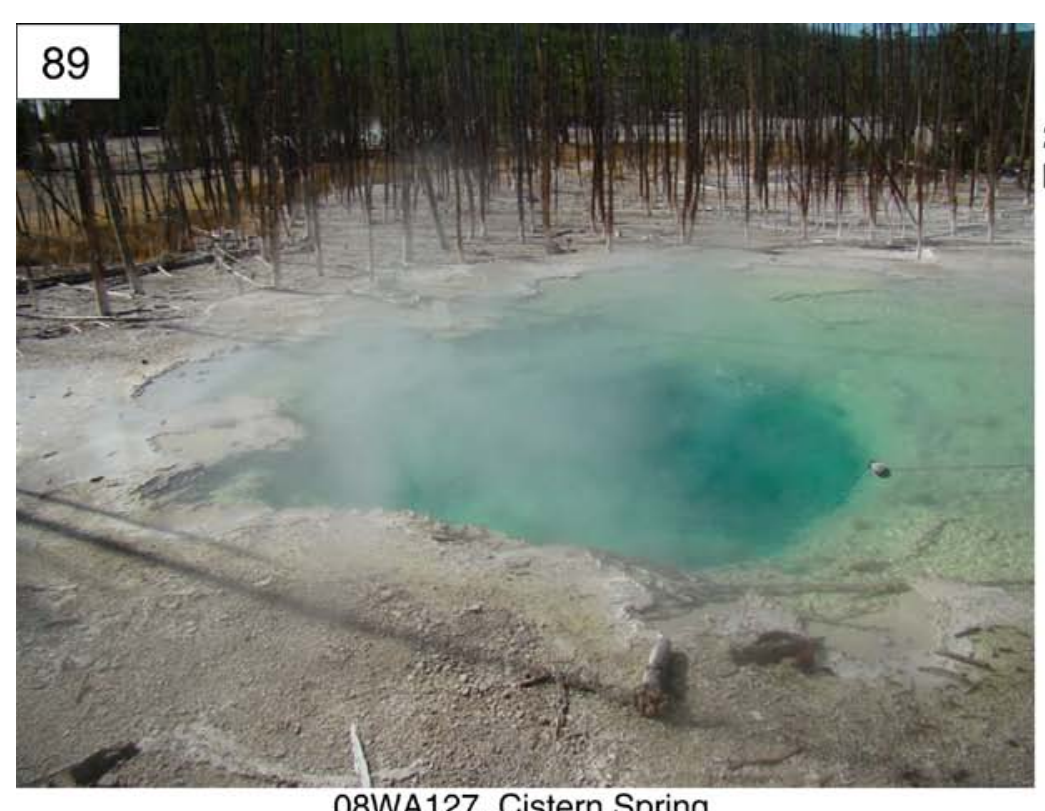

$\bar{\infty}$

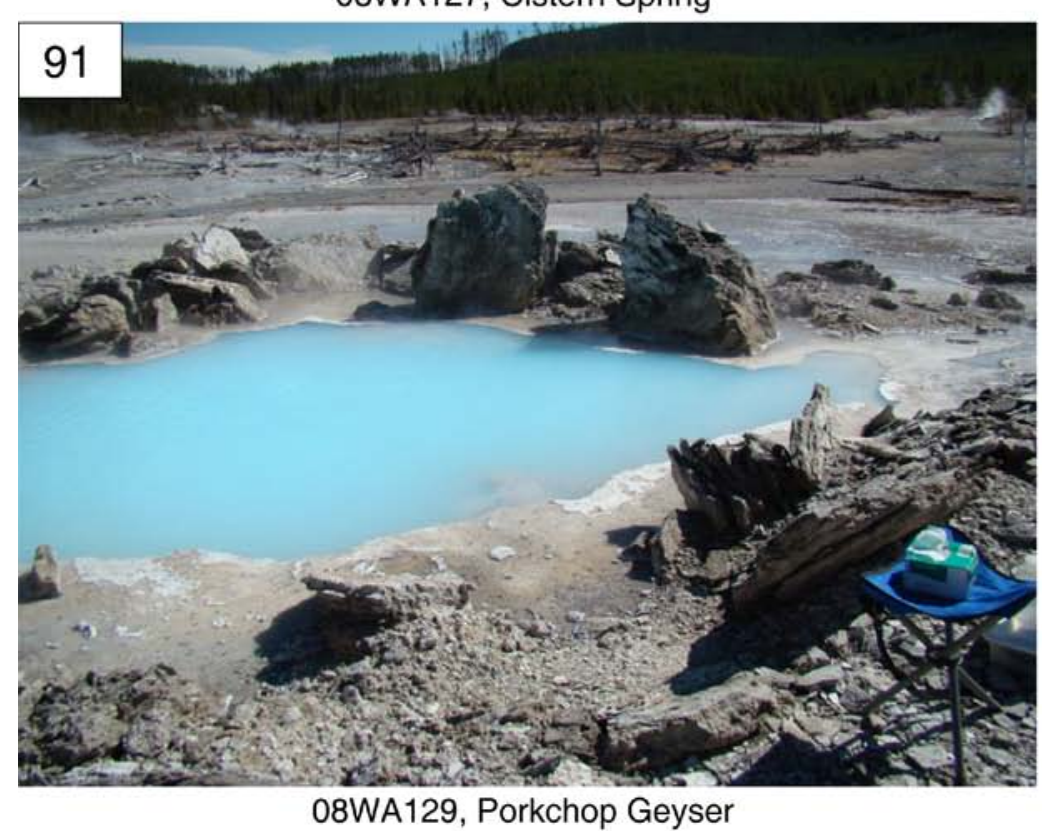

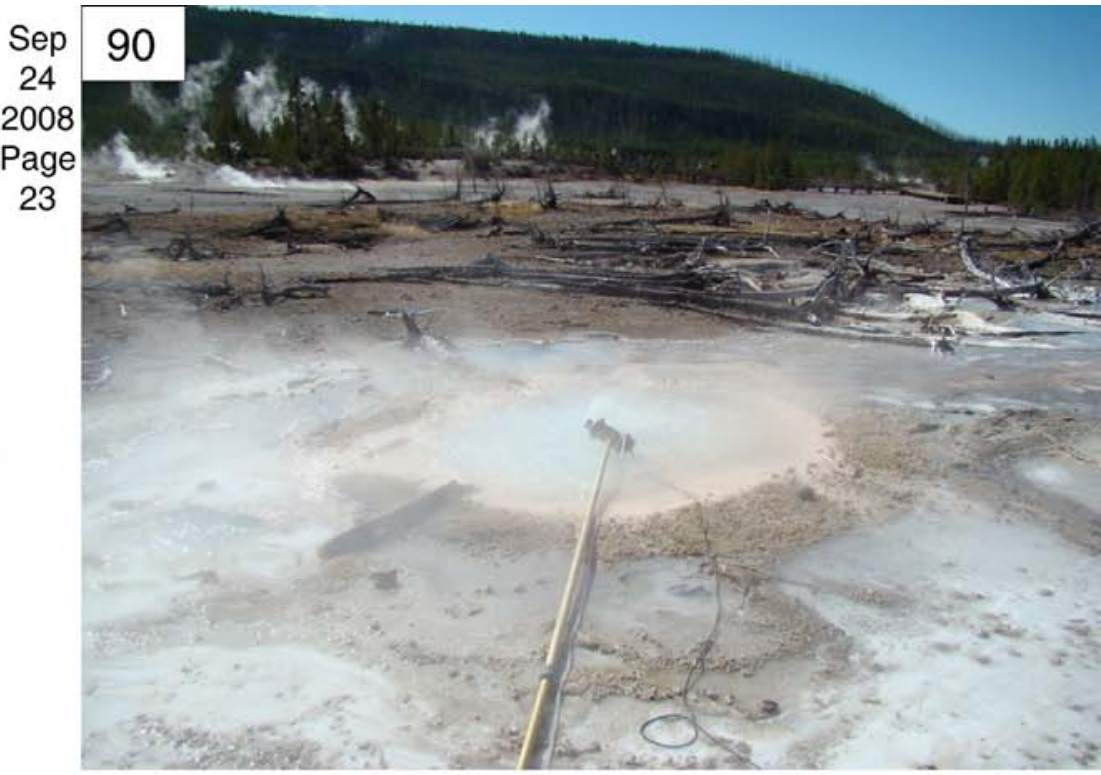

08WA128, "Second Eruptor"

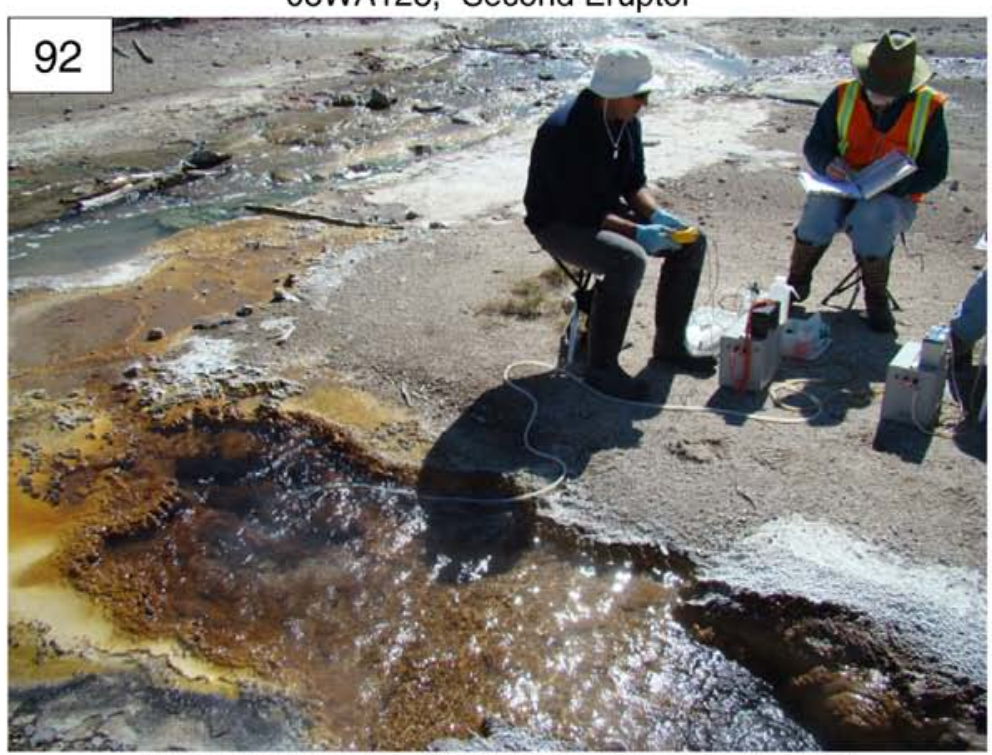

Nuno Durães and Kirk Nordstrom measuring pH at 08WA130, Perpetual Spouter 

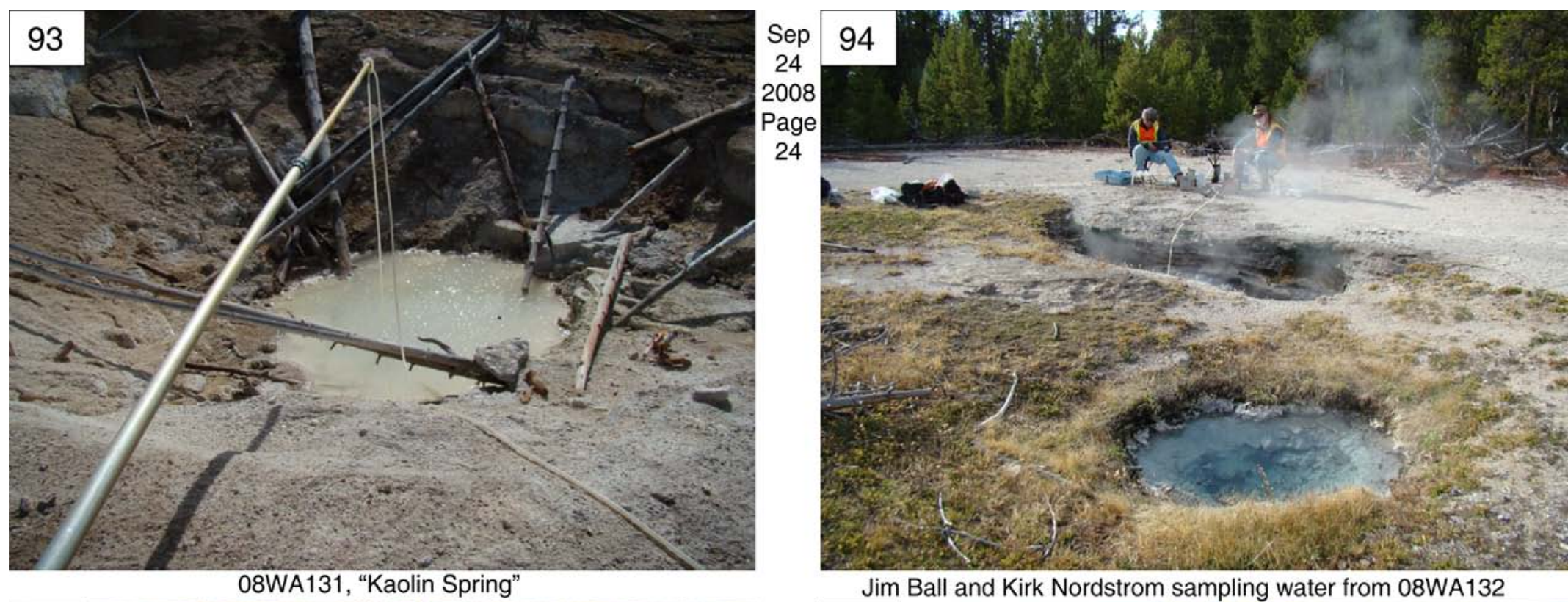

용

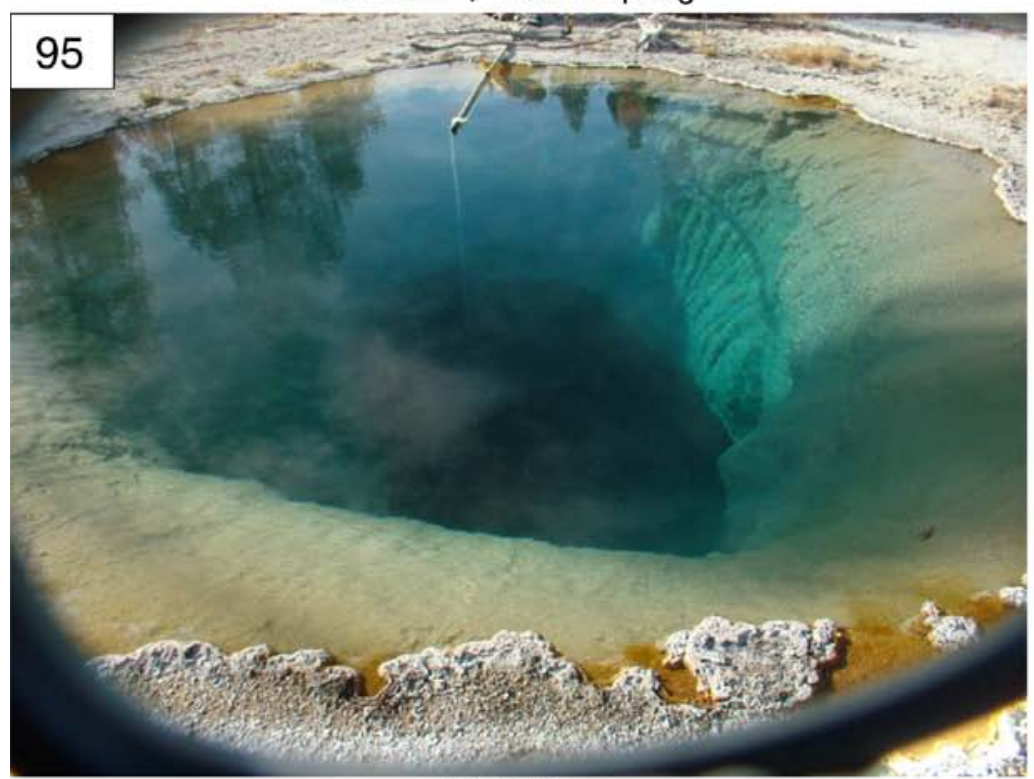

Jim Ball and Kirk Nordstrom sampling water from 08WA132

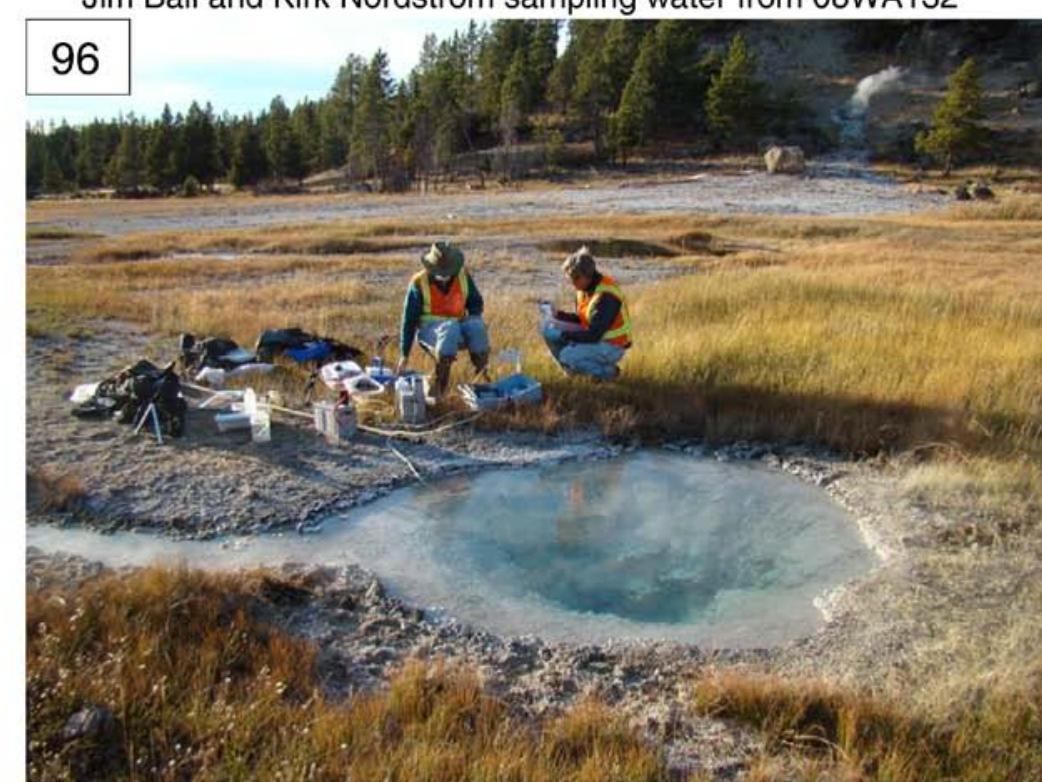

Kirk Nordstrom and Jim Ball Sampling water from 08WA134 


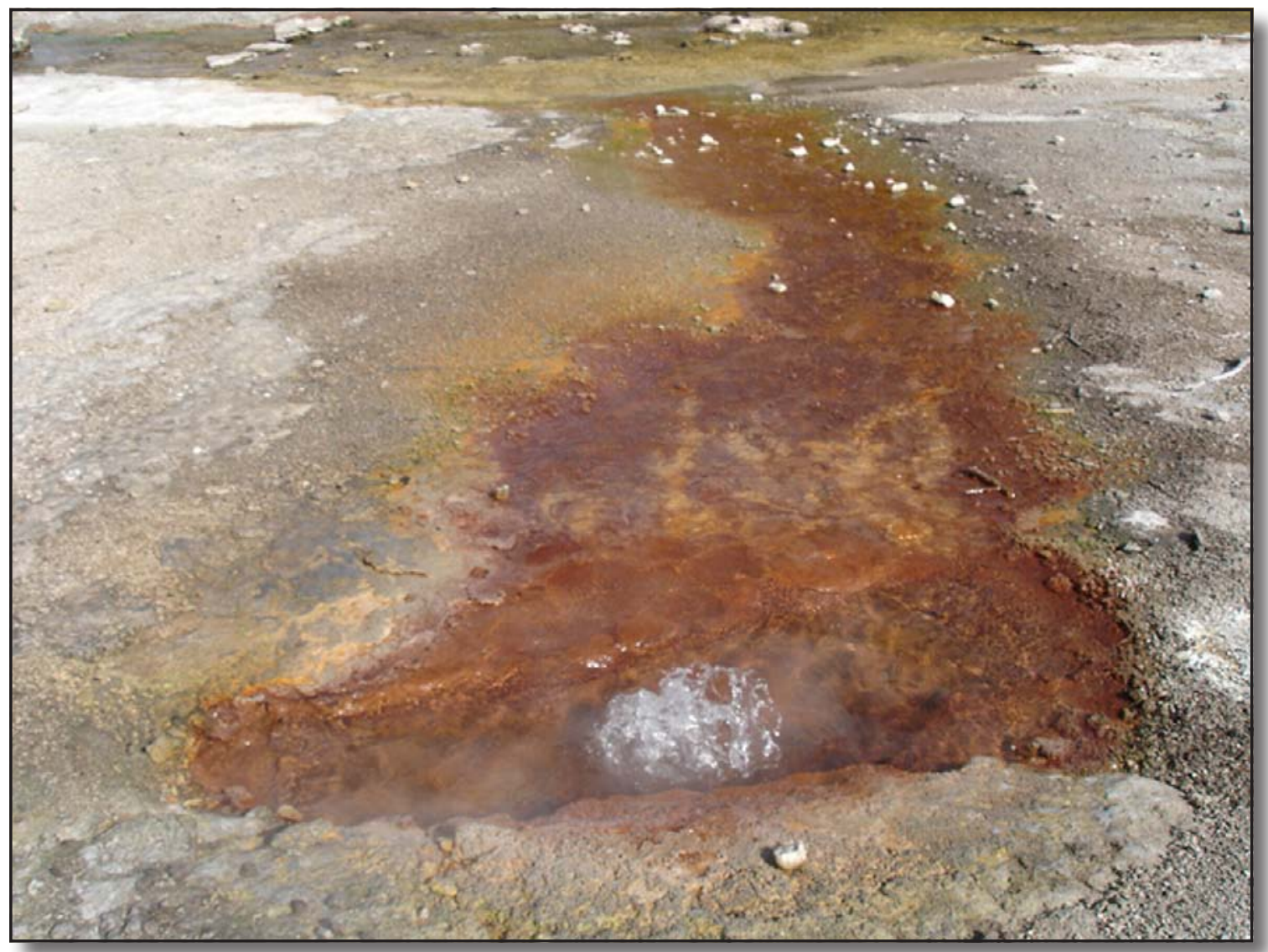

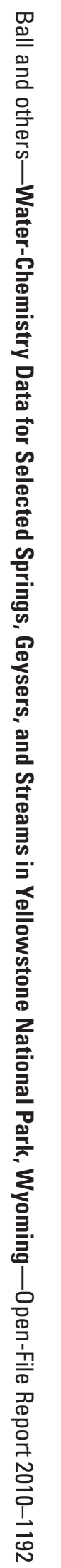

